\title{
EXPLORATION OF FINITE DIMENSIONAL KAC ALGEBRAS AND LATTICES OF INTERMEDIATE SUBFACTORS OF IRREDUCIBLE INCLUSIONS
}

\author{
MARIE-CLAUDE DAVID AND NICOLAS M. THIÉRY
}

\begin{abstract}
We study the four infinite families $K A(n), K B(n), K D(n), K Q(n)$ of finite dimensional Hopf (in fact Kac) algebras constructed respectively by A. Masuoka and L. Vainerman: isomorphisms, automorphism groups, self-duality, lattices of coideal subalgebras. We reduce the study to $K D(n)$ by proving that the others are isomorphic to $K D(n)$, its dual, or an index 2 subalgebra of $K D(2 n)$. We derive many examples of lattices of intermediate subfactors of the inclusions of depth 2 associated to those Kac algebras, as well as the corresponding principal graphs, which is the original motivation.

Along the way, we extend some general results on the Galois correspondence for depth 2 inclusions, and develop some tools and algorithms for the study of twisted group algebras and their lattices of coideal subalgebras. This research was driven by heavy computer exploration, whose tools and methodology we describe.
\end{abstract}

RÉSumÉ. Nous étudions les quatre familles $K A(n), K B(n), K D(n), K Q(n)$ d'algèbres de Hopf (en fait de Kac) de dimension finie construites respectivement par A. Masuoka et L. Vainerman : isomorphismes, groupes d'automorphismes, autodualité, treillis des sousalgèbres coidéales. Nous réduisons l'étude à $K D(n)$ en démontrant que les autres algèbres sont toutes isomorphes à $K D(n)$, à sa duale ou à une sous-algèbre d'indice 2 de $K D(2 n)$. Nous en déduisons de nombreux exemples de treillis de facteurs intermédiaires d'inclusions de profondeur 2 associées à ces algèbres de Kac, ainsi que les graphes principaux correspondants.

$\mathrm{Au}$ cours de cette étude, nous approfondissons des résultats généraux sur la correspondance de Galois pour les inclusions de profondeur 2 et donnons des méthodes et algorithmes pour l'analyse des algèbres de groupes déformées et leurs treillis des sous-algèbres coidéales. Cette recherche a été guidée par une exploration informatique intensive, dont nous décrivons les outils et la méthodologie.

Date: October 29, 2018.

2010 Mathematics Subject Classification. Primary 16T05; Secondary 46L37, 46L65, 16-04.

Key words and phrases. Finite dimensional Kac algebras, Hopf algebras, quantum groupoids, lattices of intermediate subfactors, principal graphs, computer exploration. 


\section{Contents}

List of Figures

1. Introduction

2. Depth 2 inclusions, intermediate subfactors and coideal subalgebras

2.1. Depth 2 inclusions and $C^{*}$-quantum groupoids

2.2. Galois correspondence

2.3. The Jones projection of a coideal subalgebra

2.4. The inclusion associated with a coideal subalgebra and its Jones tower

2.5. The principal graph of an intermediate inclusion

2.6. Depth 2 intermediate inclusions

2.7. The $C^{*}$-quantum groupoids of dimension 8

2.8. Special case of irreducible inclusions

3. The Kac-Paljutkin algebra

3.1. The Kac-Paljutkin algebra $K P$

3.2. The coideal subalgebras of $K P$

3.3. The lattice $1(K P)$

3.4. Realization of $K P$ by composition of subfactors

4. Principal graphs obtained in our examples

4.1. Graphs $D_{2 n+1} / \mathbb{Z}_{2}$

4.2. Graphs $D_{2 n+2} / \mathbb{Z}_{2}$

4.3. Graphs $D_{n}^{(1)}$

4.4. Graphs $Q B_{n}$

4.5. Graphs $D B_{n}$

5. The Kac algebras $K D(n)$ and twisted group algebras

5.1. Twisted group algebras

5.2. Notations

5.3. The Kac algebra $K D(2)$ of dimension 8

5.4. Coidalgebras induced by subgroups

5.5. Automorphism group

5.6. The three coideal subalgebras $K_{2}, K_{3}, K_{4}$ of dimension $2 n$

5.7. The Kac subalgebra $K_{2}=I\left(e_{1}+e_{2}\right)$ of dimension $2 n$

5.8. The coideal subalgebras of $K_{2}$

5.9. The coideal subalgebra $K_{1}=I\left(e_{1}+e_{2}+e_{3}+e_{4}\right)$ of dimension $n$

5.10. Coidalgebras of dimension dividing $2 n$

5.11. Coidalgebras induced by Jones projections of subgroups

6. The Kac algebras $K D(n)$ for $n$ odd

6.1. Self-duality

6.2. The symmetric Kac subalgebras

6.3. The other coideal subalgebras of dimension $2 n$ : $K_{3}$ and $K_{4}$

6.4. The coideal subalgebra $K_{1}=I\left(e_{1}+e_{2}+e_{3}+e_{4}\right)$ of dimension $n$

6.5. The coideal subalgebras of dimension dividing $n$

6.6. The coideal subalgebras of $K_{3}$ and $K_{4}$

6.7. The coideal subalgebras of dimension 4

6.8. The lattice $\mathrm{l}(K D(n))$

6.9. The Kac algebra $K D(3)$ of dimension 12

6.10. The algebra $K D(9)$ of dimension 36

6.11. The algebra $K D(15)$ of dimension 60

7. The Kac algebras $K D(n)$ for $n$ even

7.1. The algebra of the intrinsic group $K_{0}$ 
7.2. The Kac subalgebra $K_{4}=I\left(e_{1}+e_{4}\right)$

7.3. The Kac subalgebra $K_{3}=I\left(e_{1}+e_{3}\right)$

7.4. The Kac subalgebra $K_{1}=I\left(e_{1}+e_{2}+e_{3}+e_{4}\right)$

7.5. The coideal subalgebras of dimension 4

7.6. The Kac algebra $K D(4)$ of dimension 16

7.7. The Kac algebra $K D(6)$ of dimension 24

8. The Kac algebras $K Q(n)$

8.1. Definition and general properties

8.2. The isomorphism $\phi$ from $K D(2 m)$ to $K Q(2 m)$

8.3. Self-duality for $n$ odd

8.4. The lattice $1(K Q(n))$ for $n$ odd

9. The Kac algebras $K B(n)$ and $K_{3}$ in $K D(2 m)$

9.1. The subalgebra $K_{3}$ of $K D(2 m)$

9.2. $K D(n), K Q(n), K A(n)$, and $K B(n)$ : isomorphisms, coideal subalgebras of dimension $2 n$

9.3. The lattice $1(K B(m))$

Appendix A. Collection of formulas for $K D(n)$

A.1. Left regular representation of $D_{2 n}$

A.2. Matrix units in the group basis and coinvolution

A.3. The unitary 2-cocycle $\Omega$

A.4. Twisted coproduct of the Jones projection of the subgroup $H_{r}$

A.5. Coproducts of Jones projections for coideal subalgebras of dimension $2 n$

A.6. Coproducts of $v$ and $w$ of $K_{3}$ in $K D(2 m)$

A.7. Structure of $K_{33}$ in $K_{3}$ of $K D\left(4 m^{\prime}\right)$

A.8. Calculations for Theorem 6.1

Appendix B. Computer exploration with MuPAD-Combinat

B.1. Software context

B.2. Setup

B.3. Computing with elements

B.4. Computing with coideal subalgebras

B.5. Computing Kac isomorphisms and applications

B.6. Further directions

References 


\section{List OF FiguRES}

1 The lattice of coideal subalgebras of $K P \quad 20$

2 The graph $D_{11} / \mathbb{Z}_{2}$

3 The graph $D_{12} / \mathbb{Z}_{2}$

4 The graph $D_{6}^{(1)} 23$

5 The graph $Q B_{2} \quad 23$

6 The graph $D B_{2}$

7 The lattice of coideal subalgebras of $K D(2) \quad 25$

8 The lattice of coideal subalgebras of $K D(5) \quad 39$

9 The lattice of coideal subalgebras of $K D(3)$

10 The lattice of coideal subalgebras of $K D(9)$

11 The lattice of coideal subalgebras of $K D(15) \quad 42$

12 The lattice of coideal subalgebras of $K D(4) \quad 46$

13 The lattice of coideal subalgebras of $K D(6)$

14 The lattice of coideal subalgebras of $K Q(3) \quad 56$

15 The lattice of coideal subalgebras of $K D(8)$ 


\section{INTRODUCTION}

The theory of Kac algebras provides a unified framework for both group algebras and their duals. In finite dimension this notion coincides with that of $C^{*}$-Hopf algebras (see 2.8.1). Those algebras play an important role in the theory of inclusions of hyperfinite factors of type $I I_{1}$; indeed, any irreducible finite index depth 2 subfactor is obtained as fixed point set under the action of some finite dimensional Kac algebra on the factor. There furthermore is a Galois-like correspondence between the lattice of intermediate subfactors and the lattice of coideal subalgebras of the Kac algebra. A cocommutative (respectively commutative) Kac algebra is the group algebra $\mathbb{C}[G]$ of some finite group $G$ (respectively its dual). We call such a Kac algebra trivial; its lattice of coideal subalgebras is simply given by the lattice of subgroups of $G$.

In [Wat96], Yasuo Watatani initiates the study of the lattices of irreducible subfactors of type $I I_{1}$ factors. He provides general properties as well as examples. In particular, his work, completed by Michael Aschbacher [Asc08], shows that every lattice with at most six elements derives from group actions and operations on factors.

Our aim is to study more involved examples of lattices of subfactors, and therefore of principal graphs. It is our hope that this will contribute to the general theory by providing a rich ground for suggesting and testing conjectures. A secondary aim is to evaluate the potential of computer exploration in that topic, to develop algorithms and practical tools, and to make them freely available for future studies.

We always assume the factors to be hyperfinite of type $I I_{1}$, and the inclusions to be of finite index. Our approach is to use the Galois correspondence and study instead the lattices of coideal subalgebras of some non trivial examples of Kac algebras. Only lattices of depth 2 irreducible inclusions of type $I I_{1}$ hyperfinite factors are obtained this way. The depth 2 hypothesis is not so restrictive though, since D. Nikshych and L. Vainerman showed in [NV00c] that any finite depth inclusion is an intermediate subfactor of a depth 2 inclusion. There remains only the irreducibility condition. Luckily, there still exists a Galois correspondence for non irreducible depth 2 inclusions, at the price of considering the action of a finite $C^{*}$-quantum groupoid instead of a Kac algebra (see NV00b, Dav05, Dav09). The study of more general examples coming from $C^{*}$-quantum groupoids, like those constructed from tensor categories by C. Mével, is the topic of subsequent work.

The first non trivial example of Kac algebra KP was constructed in 1966 (see [KP66]); it is the unique one of dimension 8. Later, M. Izumi and H. Kosaki classified small dimension Kac algebras through factors (see [IK02]). In 1998, L. Vainerman constructed explicitly two infinite families of Kac algebras, which we denote $K D(n)$ and $K Q(n)$, by deformation of the group algebras of the dihedral groups $D_{2 n}$ and of the quaternion groups $Q_{2 n}$ respectively (see [Vai98 [1). In 2000, A. Masuoka defined two other infinite families $A_{4 n}$ and $B_{4 n}$ (see [Mas00, def 3.3]), which we denote $K A(n)$ and $K B(n)$ for notational consistency.

We present here a detailed study of the structure of those Kac algebras, with an emphasis on $K D(n)$. We get some structural results: $K Q(n)$ is isomorphic to $K D(n)$ for $n$ even and to $K B(n)$ for $n$ odd, while $K B(n)$ itself is an index 2 Kac subalgebra of $K D(2 n)$; also, $K A(n)$ is the dual of $K D(n)$. Altogether, this is the rationale behind the emphasis on $K D(n)$. We prove that $K D(n)$ and $K Q(n)$ are self-dual if (and only if) $n$ is odd, by constructing an explicit isomorphism (the self-duality of $K A(n)$ for $n$ odd and of $K B(n)$ for all $n$ is readily proved in CDMM04]). We also describe the intrinsic groups and automorphism groups of $K D(n)$ and $K Q(n)$ (see CDMM04 for those of $K A(n)$ and $K B(n))$.

\footnotetext{
${ }^{1}$ Note that Vai98 is a follow up on a study initiated in 1996 with M. Enock EV96. An analogous construction can be found in Nik98.
} 
Then, we turn to the study of the lattice of coideal subalgebras of those Kac algebras. We describe them completely for $n$ small or prime, and partially for all $n$. For $K D(n)$, we further obtain a conjectural complete description of the lattice for $n$ odd, and explain how large parts can be obtained recursively for $n$ even. After this study, the lattice of intermediate subfactors of all irreducible depth 2 inclusions of index at most 15 is known. We derive the principal graphs of certain inclusions of factors; reciprocally we use classification results on inclusions in some proofs.

As the first interesting examples are of dimension 12 or more, calculations are quickly impractical by hand. Most of the research we report on in this paper has been driven by computer exploration: it led to conjectures and hinted to proofs. Furthermore, whenever possible, the lengthy calculations required in the proofs were delegated to the computer. Most of the tools we developed for this research (about 8000 lines of code) are generic and integrated in the open source MuPAD-Combinat package, and have readily been reused to study other algebras.

In Section 2, we recall and refine the results of [NV00c], and adapt them to the duality framework used in Dav05]. In order to build the foundations for our future work on $C^{*}$-quantum groupoids, the results are first given in this general setting, at the price of some technical details in the statements and proofs. The results which are used in the subsequent sections are then summarized and further refined in 2.8 in the simpler context of Kac algebras.

In Section 3, we describe the complete lattice of coideal subalgebras of the smallest non trivial Kac algebra, KP, using the construction of [EV96]. In Section 4, we gather some graphs that are obtained as principal graphs of intermediate subfactors.

In Section 5, we give general results on $K D(n)$. We describe its intrinsic group and its automorphism group; more generally, we describe the embeddings of $K D(d)$ into $K D(n)$. Along the way, we build some general tools for manipulating twisted group algebras (isomorphism computations and proof strategies, coideal subalgebras induced by subgroups, efficient characterization of the algebra of the intrinsic group). Those tools will be reused extensively in the later sections. We list the three dimension $2 n$ coideal subalgebras: $K_{2}$, $K_{3}$, and $K_{4}$, and further prove that $K_{2}$ is isomorphic to $L^{\infty}\left(D_{n}\right)$. We also describe some coideal subalgebras induced by Jones projections of subgroups of $D_{2 n}$, as well as how to obtain recursively coideal subalgebras of dimension dividing $2 n$.

The properties of $K D(n)$ depend largely on the parity of $n$. In Section 6 , we concentrate on the case $n$ odd. We show that $K D(n)$ is then self-dual, by constructing an explicit isomorphism with its dual. We present a partial description of the lattice $1(K D(n))$ which is conjecturally complete. This is proved for $n$ prime and checked on computer up to $n=51$. We conclude with some illustrations on $K D(n)$ for $n=3,5,9,15$.

In Section 7, we consider $K D(2 m)$. We prove that $K_{3}$ and $K_{4}$ are Kac subalgebras. In fact $K_{4}$ is isomorphic to $K D(m) ; K_{3}$ is further studied in Section 9 . It follows that a large part of the lattice of coideal subalgebras of $K D(2 m)$ can be constructed inductively. We list the dimension 4 coideal subalgebras of $K D(2 \mathrm{~m})$, and describe the complete lattice of coideal subalgebras for $K D(4)$ and $K D(6)$.

In Section 8, we briefly study the algebra $K Q(n)$. Its structure mimics that of $K D(n)$. In fact, we prove that $K Q(n)$ is isomorphic to $K D(n)$ if and only if $n$ is even and that $K Q(n)$ is self-dual when $n$ is odd. We give the complete lattice for $K Q(n)$ for $n$ prime, and list the coideal subalgebras of dimension $2,4, n$, and $2 n$, for all $n$. Those results are used in the previous sections.

In Section 9, we show that the Kac subalgebra $K_{3}$ of $K D(2 m)$ is isomorphic to $K B(m)$. We summarize the links between the families $K A(n), K B(n), K D(n)$, and $K Q(n)$, and the coideal subalgebras of dimension $4 n$ in $K D(2 n)$. We list the coideal subalgebras of 
dimension $2,4, m$, and $2 m$ of $K_{3}$ in $K D(2 m)$ and describe the complete lattice of coideal subalgebras for $K D(8)$.

In Appendix A, we collect various large formulas for matrix units, coproducts, unitary cocycles, etc. which are used throughout the text, and we finish some technical calculations for the proof of Theorem 6.1.

Finally, in Appendix B, we quickly describe the computer exploration tools we designed, implemented, and used, present typical computations, and discuss some exploration strategies.

\section{ACKNOWLEDGMENTS}

We would like to thank Vaughan Jones for his initial suggestion of investigating concrete examples of lattices of intermediate subfactors, and for fruitful questions. We are also very grateful to Leonid Vainerman for his continuous support and his reactivity in providing wise suggestions and helpful answers.

This research was partially supported by NSF grant DMS-0652641, and was driven by computer exploration using the open-source algebraic combinatorics package MuPADCombinat [HT04]. 


\section{DEPTH 2 INCLUSIONS, INTERMEDIATE SUBFACTORS AND COIDEAL SUBALGEBRAS}

In this section, we recall and refine the general results of D. Nikshych et L. Vainerman [NV00c] NV00c] on depth 2 inclusions and their Galois correspondence.

In order to build the foundations for our future work on finite dimensional $C^{*}$-quantum groupoids, we do not assume here the inclusion to be irreducible. Except for some technicalities, this does not affect the theory. For the convenience of the reader, the results which are used in the subsequent sections are summarized and further refined in 2.8 in the simpler context of Kac algebras. This mostly amounts to replacing $C^{*}$-quantum groupoids by Kac algebra, and setting $h=1$.

For the general theory of subfactors, we refer to [Jon83, GdlHJ89] et JS97].

\subsection{Depth 2 inclusions and $C^{*}$-quantum groupoids.}

2.1.1. $C^{*}$-quantum groupoids. A finite $C^{*}$-quantum groupoid is a weak finite dimensional $C^{*}$-Hopf algebra: the image of the unit by the coproduct is not required to be $1 \otimes 1$, and the counit is not necessarily an homomorphism (see [BNS99, [NV00b] or Dav05, 2.1]). More precisely:

Definition 2.1.1. A finite $C^{*}$-quantum groupoid is a finite dimensional $C^{*}$-algebra $G$ (we denote by $m$ the multiplication, by 1 the unit, and by ${ }^{*}$ the involution) endowed with an coassociative coalgebra structure (we denote by $\Delta$ the coproduct, $\varepsilon$ the counit and $S$ the antipode) such that:

(i) $\Delta$ is a *-algebra homomorphism from $G$ to $G \otimes G$ satisfying:

$$
(\Delta \otimes \mathrm{id}) \Delta(1)=(1 \otimes \Delta(1))(\Delta(1) \otimes 1)
$$

(ii) The counit is a linear map of $G$ to $\mathbb{C}$ satisfying:

$$
\varepsilon(f g h)=\varepsilon\left(f g_{(1)}\right) \varepsilon\left(g_{(2)} h\right) \quad\left((f, g, h) \in G^{3}\right)
$$

Equivalently:

$$
\left.\varepsilon(f g h)=\varepsilon\left(f g_{(2)}\right) \varepsilon\left(g_{(1)} h\right) \quad\left((f, g, h) \in G^{3}\right)\right)
$$

(iii) The antipode $S$ is an antiautomorphism of algebra and coalgebra of $G$ satisfying for all $g$ in $G$ :

$$
m(\mathrm{id} \otimes S) \Delta(g)=(\varepsilon \otimes \mathrm{id})(\Delta(1)(g \otimes 1))
$$

or equivalently:

$$
m(S \otimes \mathrm{id}) \Delta(g)=(\mathrm{id} \otimes \varepsilon)((1 \otimes g) \Delta(1))
$$

The target counital map $\varepsilon_{t}$ and source counital map $\varepsilon_{s}$ are the idempotent homomorphisms defined respectively for all $g \in G$ by:

$$
\varepsilon_{t}(g)=(\varepsilon \otimes \mathrm{id})(\Delta(1)(g \otimes 1)) \quad \text { and } \quad \varepsilon_{s}(g)=(\mathrm{id} \otimes \varepsilon)((1 \otimes g) \Delta(1))
$$

2.1.2. $C^{*}$-quantum groupoid associated to an inclusion. Let $N_{0} \subset N_{1}$ be a depth 2 inclusion of $\mathrm{II}_{1}$ factors of finite index $\tau^{-1}$. Consider the tower

$$
N_{0} \subset N_{1} \stackrel{f_{1}}{\subset} N_{2} \stackrel{f_{2}}{\subset} N_{3} \subset \cdots
$$

obtained by basic construction. We denote by $t r$ the unique normalized trace of the factors. The relative commutants $A=N_{0}^{\prime} \cap N_{2}$ and $B=N_{1}^{\prime} \cap N_{3}$ are endowed with dual finite regular $C^{*}$-quantum groupoid structures thanks to the duality:

$$
\langle a, b\rangle=\left[N_{1}: N_{0}\right]^{2} \operatorname{tr}\left(a h f_{2} f_{1} h b\right) \quad(a \in A, b \in B)
$$

\footnotetext{
${ }^{2} S^{2}$ is the identity on the counital algebras.
} 
where $h$ is the square root of the index of the restriction of the trace $\operatorname{tr}$ to $N_{1}^{\prime} \cap N_{2}$ (see [NV00b] or [Dav05, 3.2]).

The quantum groupoid $B$ acts on $N_{2}$ in such a way that $N_{3}$ is isomorphic to the crossed product $N_{2} \rtimes B$ and $N_{1}$ is isomorphic to the subalgebra of the points of $N_{2}$ fixed under the action of $B$. We recall that the inclusion is irreducible if and only if $B$ is a Kac algebra. We refer to NV00b for historical notes on the problem and references to analogous results.

We have shown in Dav05 that any finite $C^{*}$-quantum groupoid $B$ is isomorphic to a regular $C^{*}$-quantum groupoid and acts on the $I I_{1}$ hyperfinite factor $R$. If $A$ and $B$ are two dual finite regular $C^{*}$-quantum groupoids, we can slightly modify the construction of Dav05 so that the obtained tower of $I I_{1}$ hyperfinite factors $N_{0} \subset N_{1} \subset N_{2} \subset N_{3}$ endows the relative commutants $M_{0}^{\prime} \cap M_{2}$ and $M_{1}^{\prime} \cap M_{3}$ (respectively isomorphic to $A$ and $B$ as $C^{*}$-algebras) with dual finite regular $C^{*}$-quantum groupoid structures which are respectively isomorphic to the original ones (see [Dav09]).

Therefore we may, without loss of generality, reduce our study to finite regular $C^{*}$ quantum groupoids associated to an inclusion.

In this context, there are convenient expressions for the counit, the source and target counital maps, the antipodes, and the action: for any element $b \in B$, they satisfy:

$$
\begin{array}{clrl}
\varepsilon_{B}(b)=\tau^{-1} \operatorname{tr}\left(h f_{2} h b\right), & S_{B}(b)=h j_{2}\left(h^{-1}\right) j_{2}(b) h^{-1} j_{2}(h), \\
\varepsilon_{B}^{t}(b)=\tau^{-1} E_{N_{1}^{\prime} \cap N_{2}}\left(b h f_{2} h^{-1}\right), & \varepsilon_{B}^{s}(b)=\tau^{-1} E_{N_{2}^{\prime} \cap N_{3}}\left(j_{2}(b) h^{-1} f_{2} h\right),
\end{array}
$$

where, for any $n \in \mathbb{N}, j_{n}$ is the antiautomorphism of $N_{0}^{\prime} \cap N_{2 n}$ defined by:

$$
j_{n}(x)=J_{n} x^{*} J_{n} \quad \text { for } x \in N_{0}^{\prime} \cap N_{2 n},
$$

where $J_{n}$ is the canonical anti-unitary involution of $L^{2}\left(N_{n}, t r\right)$, space of the GNS construction of $N_{n}$ [Dav05, 3.1].

The application $S_{B}^{2}$ is the inner automorphism defined by $G=j_{2}\left(h^{-2}\right) h^{2}$.

The action of $B$ on $N_{2}$ is defined by:

$$
b \triangleright x=\tau^{-1} E_{N_{2}}\left(b x h f_{2} h^{-1}\right) \quad \text { for } x \in N_{2}, b \in B .
$$

There are analogous formulas for $A$.

The algebra $N_{0}$ is then the algebra of the points of $N_{1}$ which are fixed under the action of the $C^{*}$-quantum groupoid $A$. The application $\Theta_{A}:[x \otimes a] \longmapsto x a$ is a von Neumann algebra isomorphism from $N_{1} \rtimes A$ to $N_{2}$. The application $\Theta_{B}$ from $N_{2} \rtimes B$ to $N_{3}$ is defined similarly. In the sequel, we mostly consider the $C^{*}$-quantum groupoid $B$, and then set $\Theta=\Theta_{B}$ for short.

2.2. Galois correspondence. In NV00c, D. Nikshych and L. Vainerman show that the correspondence between finite index depth 2 inclusions and finite $C^{*}$-quantum groupoids is a Galois-type correspondence.

\subsubsection{Involutive left coideal subalgebra.}

Definition 2.2.1. An involutive left (resp. right) coideal $*$-subalgebra (or coideal subalgebra for short) $I$ of $B$ is a unital $C^{*}$-subalgebra of $B$ such that $\Delta(I) \subset B \otimes I$ (resp. $\Delta(I) \subset I \otimes B)$.

As in [NV00c, 3.1], the lattice of left (resp. right) coideal subalgebra of $B$ is denoted by $\mathrm{l}(B)($ resp. $\mathrm{r}(B))$.

2.2.2. Cross product by a coideal subalgebra. By the definition of a coideal subalgebra, the image of $N_{2} \otimes I$ by $\Theta$ is a von Neumann subalgebra $M_{3}$ of $N_{3}$, called cross product of $N_{2}$ by $I$ and denoted by $M_{3}=N_{2} \rtimes I$. 
2.2.3. Intermediate subalgebras of an inclusion. Let $\mathrm{l}\left(N_{2} \subset N_{3}\right)$ be the lattice of intermediate subalgebras of an inclusion $N_{2} \subset N_{3}$, that is von Neumann algebras $M_{3}$ such that $N_{2} \subset M_{3} \subset N_{3}$.

In theorem 4.3 of $\mathrm{NV} 00 \mathrm{c}$, D. Nikshych and L. Vainerman establish an isomorphism between $\mathrm{l}\left(N_{2} \subset N_{3}\right)$ and $\mathrm{l}(B)$. More precisely, if $M_{3}$ is an intermediate von Neumann subalgebra of $N_{2} \subset N_{3}$, then $I=N_{1}^{\prime} \cap M_{3}$ is a coideal subalgebra of $B$. Reciprocally, if $I$ is a coideal subalgebra of $B$, then $M_{3}=N_{2} \rtimes I$ is an intermediate von Neumann subalgebra of $N_{2} \subset N_{3}$.

In [NV00c, 4.5], they prove that the intermediate von Neumann subalgebra is a factor if and only if the coideal subalgebra is connected, that is $Z(I) \cap B_{s}$ is trivial. From now on, we only consider connected coideal subalgebras.

2.2.4. Quasi-basis. The notion of quasibasis is defined in [Wat90, 1.2.2] and made explicit in the special case of depth 2 inclusions in [Dav05, 3.3].

Proposition 2.2.4. Let $\left\{c_{t} \mid t \in T\right\}$ be a family of matrix units of I, normalized by $\operatorname{tr}\left(c_{t}^{*} c_{t}^{\prime}\right)=\delta_{t, t^{\prime}}$. Then, $\left\{c_{t} h^{-1} \mid t \in T\right\}$ is a quasibasis of $M_{3} \cap N_{1}^{\prime}$ over $N_{2} \cap N_{1}^{\prime}$, and therefore a quasibasis of $M_{3}$ over $N_{2}$.

The index $\tau_{I}^{-1}$ of $N_{2}$ in $M_{3}$ is then $\sum_{t \in T} c_{t} H^{-1} c_{t}^{*}$.

Proof. By [Wat90, 2.4.1], the family $\left\{c_{t} h^{-1} \mid t \in T\right\}$ is a quasibasis of $M_{3} \cap N_{1}^{\prime}$ over $N_{2} \cap N_{1}^{\prime}$. Since $M_{3}$ is generated by $N_{2}$ and $I$, this family is a quasibasis of $M_{3}$ over $N_{2}$ (see also [Dav05, 3.3]). Since the trace of the factors is a Markov trace, the index of the conditional expectation of $M_{3}$ over $N_{2}$ is the scalar $\tau_{I}^{-1}=\sum_{t \in T} c_{t} H^{-1} c_{t}^{*}$ Wat90, 2.5.1].

Corollary 2.2.4. If the inclusion $N_{0} \subset N_{1}$ is irreducible, the family $\left\{c_{t} \mid t \in T\right\}$ of normalized matrix units of $I$ is a Pimsner-Popa basis of $M_{3}$ over $N_{2}$, and the dimension of $I$ coincides with the index of $N_{2}$ in $M_{3}$.

Proof. In this case, $h=1$. Therefore $\left[M_{3}: N_{2}\right]$ is the dimension of $I$, and the family $\left\{c_{t} \mid t \in T\right\}$ is a Pimsner-Popa basis of $M_{3}$ over $N_{2}$ (see [PP86, 1.3] and [Dav96, 5.2.1]).

\subsection{The Jones projection of a coideal subalgebra.}

2.3.1. Restriction of the counit to $I$. It has been noticed in Dav05 that the counit, the Haar projection of $B$, and the Jones projection are closely related. This phenomenon also occurs for coideal subalgebras.

Proposition 2.3.1 (Proposition [NV00c, 3.5]). The restriction of $\varepsilon$ to $I$ is a positive linear form on $I$. There therefore exists a unique positive element $x_{I}$ of $I$ such that $\varepsilon(b)=\operatorname{tr}\left(x_{I} b\right)$ for all $b$ in $I$. Then,

$$
\Delta\left(x_{I}\right)=\sum_{t \in T} S\left(c_{t}^{*}\right) G \otimes c_{t} \quad \text { et } \quad S\left(x_{I}\right)=x_{I} G^{-1} .
$$

Note that we use here a single trace, namely the normalized Markov trace which is that of the factors.

2.3.2. The Jones projection of $I$. We now extend Proposition 4.7 of [NV00c]: 
Proposition 2.3.2. The element $p_{I}=\tau_{I} h^{-1} x_{I} h^{-1}$ is a projection of I of trace $\tau_{I}$ which we call Jones projection of I. Furthermore:

$$
\begin{aligned}
p_{I} & =\tau_{I} \tau^{-1} E_{I}\left(f_{2}\right) \\
\Delta\left(p_{I}\right) & =\tau_{I} \sum_{t \in T} h^{-1} S\left(H^{-1} c_{t}^{*}\right) h \otimes c_{t}=\tau_{I} \sum_{t \in T} j_{2}\left(c_{t}^{*} h^{-1}\right) \otimes c_{t} h^{-1} \\
j_{2}\left(p_{I}\right) & =p_{I} \quad \text { et } \quad S\left(p_{I}\right)=G^{1 / 2} p_{I} G^{-1 / 2}
\end{aligned}
$$

Proof. Note that, in the proof of Proposition 4.7 of [NV00c], $\varepsilon_{s}\left(H^{-1} x_{I}\right)$ is exactly $\tau_{I}^{-1}$. That $p_{I}$ is a projection follows from the same proof. The equality $p_{I}=\tau_{I} \tau^{-1} E_{I}\left(f_{2}\right)$ follows from the uniqueness of $x_{I}$, and yields the trace of $p_{I}$. The counit restricted to $I$ can then be expressed as $\varepsilon(y)=\tau_{I}^{-1} \operatorname{tr}\left(y h p_{I} h\right)$, for all $y \in I$.

The coproduct formula is obtained using 2.2.4 and 2.3.1. The formula of 2.1.2 for the antipode gives the second expression which also involves the quasibasis (see 2.2.4).

From $S\left(x_{I}\right)=x_{I} G^{-1}$ and 2.1.2, one deduces successively

$$
j_{2}\left(x_{I}\right)=h^{-1} j_{2}(h) x_{I} h^{-1} j_{2}(h), \quad j_{2}\left(p_{I}\right)=p_{I}, \quad \text { and } \quad S\left(p_{I}\right)=G^{1 / 2} p_{I} G^{-1 / 2} .
$$

Remark 2.3.2. The projection $p_{I}$ generates $I$ as a coideal subalgebra. Furthermore, the coideal subalgebra $I$ is the linear span of the right legs of the coproduct of $p_{I}$. In general, we denote by $I(f)$ the coideal subalgebra generated by a projection $f$.

2.3.3. The Jones projection of $I$ is a Bisch projection. In Bis94b], D. Bisch gives the following partial characterization of Jones projections. Let $B P\left(N_{1}, N_{2}\right)$ be the set of projections $q$ of $N_{1}^{\prime} \cap N_{3}$ satisfying

BP1: $q f_{2}=f_{2}$;

BP2: $E_{N_{2}}(q)$ is a scalar;

BP3: $E_{N_{2}}\left(q f_{1} f_{2}\right)$ is the multiple of a projection.

Then, $B P\left(N_{1}, N_{2}\right)$ contains all the Jones projections of the intermediate subfactors of $N_{1} \subset N_{2}$ and is contained in the set of all the Jones projection of the intermediate von Neumann subalgebra of $N_{1} \subset N_{2}$.

Using this result, D. Nikshych et L. Vainerman show that $p_{I}$ is the Jones projection 3 of the intermediate inclusion $M_{1}=\left\{p_{I}\right\}^{\prime} \cap N_{2} \subset N_{2}$ of $N_{1} \subset N_{2}$ [NV00c, Proposition 4.8].

Proposition 2.3.3. ([NV00c, Proposition 4.8]) The projection $p_{I}$ belongs to $B P\left(N_{1}, N_{2}\right)$. It implements the conditional expectation on the intermediate subfactor $M_{1}=\left\{p_{I}\right\}^{\prime} \cap N_{2}$, whose index in $N_{2}$ is $\tau_{I}^{-1}$.

The upcoming proof follows that of Proposition 4.8 of [NV00c] albeit with our notations and duality. We identify $\Theta([z \otimes 1)]$ and $z$ for $z \in N_{2}$, as well as $\Theta([1 \otimes b)]$ and $b$ for $b \in B$.

Proof. Since the source and target counits satisfy $\varepsilon_{s} \circ S=S \circ \varepsilon_{t}$, from $\varepsilon_{s}\left(H^{-1} x_{I}\right)=\tau_{I}^{-1}$, follows that $\varepsilon_{t}\left(h p_{I} h^{-1}\right)=1$. By definition of the Haar projection $e_{B}=d^{-1} h f_{2} h^{-1}$ of $B$, we get $h p_{I} h^{-1} e_{B}=e_{B}$; this is property (BP1).

We use Proposition 2.3.2 to compute $E_{N_{2}}\left(p_{I}\right)$, and obtain property (BP2); indeed:

$$
E_{N_{2}}\left(p_{I}\right)=E_{N_{2}}\left(\tau_{I} \tau^{-1} E_{I}\left(f_{2}\right)\right)=\tau_{I} \tau^{-1} E_{N_{2}}\left(f_{2}\right)=\tau_{I}
$$

From Corollaries 2.2 and 3.9 of [NV00c] and from the expression of the coproduct of $p_{I}$, we deduce that, for any $b \in B, \tau_{I}^{-1} b_{(1)} \operatorname{tr}\left(h p_{I} h b_{(2)}\right)$ belongs to $I$ and coincides with $E_{I}(b)$.

\footnotetext{
${ }^{3}$ This motivates our terminology Jones projection of $I$.
} 
The element $E_{N_{2}}\left(p_{I} f_{1} f_{2}\right)$ belongs to $A$; using duality, Dav05, 3.1.3], and the expression of $E_{I}$, one get:

$$
\begin{aligned}
\left\langle E_{N_{2}}\left(p_{I} f_{1} f_{2}\right), b\right\rangle & =\tau^{-2} \operatorname{tr}\left(E_{N_{2}}\left(p_{I} f_{1} f_{2}\right) h f_{2} f_{1} h b\right)=\tau^{-1} \operatorname{tr}\left(p_{I} f_{1} f_{2} f_{1} h b j_{2}(h)\right) \\
& =\operatorname{tr}\left(p_{I} f_{1} h b j_{2}(h)\right)=\tau \operatorname{tr}\left(p_{I} h b j_{2}(h)\right) \\
& =\tau \operatorname{tr}\left(p_{I} h E_{I}\left(b j_{2}(h)\right)\right)=\tau \tau_{I}^{-1} \operatorname{tr}\left(p_{I} h b_{(1)}\right) \operatorname{tr}\left(h p_{I} h b_{(2)} j_{2}(h)\right) \\
& =\tau^{-1} \tau_{I}^{-1}\left\langle E_{N_{2}}\left(p_{I} f_{1} f_{2}\right) h^{-1}, b_{(1)}\right\rangle\left\langle h E_{N_{2}}\left(p_{I} f_{1} f_{2}\right), b_{(2)}\right\rangle \\
& =\tau^{-1} \tau_{I}^{-1}\left\langle E_{N_{2}}\left(p_{I} f_{1} f_{2}\right)^{2}, b\right\rangle .
\end{aligned}
$$

Therefore, $\tau^{-1} \tau_{I}^{-1} E_{N_{2}}\left(p_{I} f_{1} f_{2}\right)$ is an idempotent. Using $j_{2}\left(p_{I}\right)=p_{I}$ and [Dav05, 3.1.3], it is further self-adjoint:

$$
E_{N_{2}}\left(p_{I} f_{1} f_{2}\right)^{*}=E_{N_{2}}\left(f_{2} f_{1} p_{I}\right)=E_{N_{2}}\left(p_{I} f_{1} f_{2}\right) .
$$

By [Bis94a, 4.2], $p_{I}$ implements the conditional expectation on the intermediate subfactor $M_{1}=\left\{p_{I}\right\}^{\prime} \cap N_{2}$; its index in $N_{2}$ is $\tau_{I}^{-1}$ since $\tau_{I}$ is the trace of $p_{I}$.

Remark 2.3.3. (1) In Bis94b, Remark 4.4], D. Bisch notes that BP3 can be replaced by BP3': $q N_{2} q=N_{2} q$. In the depth 2 case, it is easy to show that one can also use instead BP3": $q A q=A q$. Indeed, BP3" follows from BP3' by application of $E_{N_{0}^{\prime} \cap N_{3}}$. Reciprocally, if BP3" holds, then BP3' follows easily since $N_{2}$ is spanned linearly by $N_{1} . A$ and $q$ commutes with $N_{1}$. Furthermore, for any $a \in A, p_{I} a p_{I}=$ $E_{\delta(I)}(a) p_{I}$, where $\delta(I)$ is defined in 2.4.1 and calculated in 2.4.2 (1).

(2) Using 2.4.2, the projection $\tau^{-1} \tau_{I}^{-1} E_{N_{2}}\left(p_{I} f_{1} f_{2}\right)$ can be described as the Jones projection of $\delta(I)$; indeed, in this setting and thanks to [2.3.2, one can write:

$$
\begin{aligned}
\tau^{-1} \tau_{I}^{-1} E_{N_{2}}\left(p_{I} f_{1} f_{2}\right) & =\tau^{-1} \tau_{I}^{-1} E_{N_{2}}\left(p_{I} f_{1} E_{M_{3}}\left(f_{2}\right)\right)=\tau_{I}^{-2} E_{N_{2}}\left(p_{I} f_{1} p_{I}\right) \\
& =\tau_{I}^{-2} E_{N_{2}}\left(E_{M_{1}}\left(f_{1}\right) p_{I}\right)=\tau_{I}^{-1} E_{\delta(I)}\left(f_{1}\right)=p_{\delta(I)} .
\end{aligned}
$$

2.3.4. Distinguished projection of $I$. Following Definition 3.6 of [NV00c], we denote by $e_{I}$ the support of the restriction of $\varepsilon$ on $I$, and call it the distinguished projection 4 of $I$. It is the minimal projection with the property $\varepsilon\left(e_{I} y e_{I}\right)=\varepsilon(y)$ for all $y$ of $I$. Furthermore, $e_{I}$ satisfies: $x_{I} e_{I}=e_{I} x_{I}=x_{I}, \varepsilon$ is faithful on $e_{I} I e_{I}$, and for all $b \in I$ one has $b e_{I}=$ $\varepsilon_{t}(b) e_{I}$ [NV00c, Proposition 3.7].

Proposition 2.3.4. The distinguished projection of $I$ is $e_{I}=E_{M_{1}}\left(H^{-1}\right) h p_{I} h$.

Proof. Since $h$ belongs to the center of $N_{1}^{\prime} \cap N_{2}$, one has: $E_{M_{1}}\left(h^{-1}\right)=E_{M_{1}}(h)^{-1}$. Let us show first some properties of $e=E_{M_{1}}\left(H^{-1}\right) h p_{I} h$. Since $p_{I}$ implements the conditional expectation on $M_{1}$, e is a projection which satisfies $\varepsilon(e y e)=\varepsilon(y)$ for $y$ in $I$ and $x_{I} e=$ $e x_{I}=x_{I}$. From the formula for $\varepsilon_{t}$ we derive:

$$
\begin{aligned}
\varepsilon_{t}(e) & =\tau^{-1} E_{N_{1}^{\prime} \cap N_{2}}\left(E_{M_{1}}(H)^{-1} h p_{I} H f_{2} h^{-1}\right) \\
& =\tau^{-1} E_{N_{1}^{\prime} \cap N_{2}}\left(E_{M_{1}}(H)^{-1} h p_{I} H p_{I} f_{2} h^{-1}\right) \\
& =\tau^{-1} E_{N_{1}^{\prime} \cap N_{2}}\left(h f_{2} h^{-1}\right)=1,
\end{aligned}
$$

\footnotetext{
${ }^{4}$ It could be called Haar projection, since it satisfies all the relevant properties
} 
as well as, for all $y \in I$ :

$$
\begin{aligned}
\varepsilon_{t}(y) e & =\tau^{-1} E_{N_{1}^{\prime} \cap N_{2}}\left(y h f_{2} h^{-1} E_{M_{1}}\left(H^{-1}\right) h\right) p_{I} E_{M_{1}}\left(H^{-1}\right) h \\
& =\tau^{-1} E_{N_{1}^{\prime} \cap N_{2}}\left(y h f_{2} p_{I}\right) p_{I} E_{M_{1}}\left(H^{-1}\right) h \\
& =\tau^{-1} \tau_{I} y h E_{I}\left(f_{2}\right) p_{I} E_{M_{1}}\left(H^{-1}\right) h \\
& =y E_{M_{1}}\left(H^{-1}\right) h p_{I} h=y e .
\end{aligned}
$$

On the other hand, from $e_{I}=\varepsilon_{t}\left(e_{I}\right) e_{I}$ we deduce successively:

$$
x_{I}=\varepsilon_{t}\left(e_{I}\right) x_{I}, \quad \varepsilon_{t}\left(H^{-1} x_{I}\right)=\tau_{I}^{-1}, \quad \text { and } \quad \varepsilon_{t}\left(e_{I}\right)=1 .
$$

Since $e e_{I}=\varepsilon_{t}(e) e_{I}=e_{I}$, we can write

$$
e_{I}=e e_{I}=e_{I} e=\varepsilon_{t}\left(e_{I}\right) e=e .
$$

\subsection{The inclusion associated with a coideal subalgebra and its Jones tower.}

2.4.1. The antiisomorphism $\delta$. In [NV00c, Proposition 3.2], D. Nikshych and L. Vainerman define a lattice isomorphism from $\mathrm{l}(B)$ to $\mathrm{r}(B)$ by $I \longmapsto \tilde{I}=G^{-1 / 2} S_{B}(I) G^{1 / 2}$. This isomorphism is induced by $j_{2}$; indeed, $\tilde{I}$ is simply $j_{2}(I)$. Note that $j_{2}(I)$ is the linear span of the left legs of $\Delta\left(p_{I}\right)$, as well as the right coideal subalgebra generated by $p_{I}$.

Following [NV00c, Proposition 3.3], consider $B$ as embedded into $A \rtimes B=N_{0}^{\prime} \cap N_{3}$, and for $I$ in $\mathrm{l}(B)$, denote by $\delta(I)$ the coideal subalgebra $j_{2}(I)^{\prime} \cap A$ of $A$. Since $j_{2}(I)$ is the right coideal subalgebra generated by $p_{I}, \delta(I)$ is the commutant of $p_{I}$ in $A$.

The map $\delta$ is a lattice antiisomorphism from $\mathrm{l}(B)$ to $\mathrm{l}(A)$ [NV00c, Proposition 3.3]. In particular, $1(B)$ is self-dual whenever $B$ is. In this case, we identify $\delta(I)$ and its image in $B$ via some isomorphism from $A$ to $B$. Note that this is slightly abusive, as this is only defined up to an automorphism of $B$.

2.4.2. The Jones tower. We keep the notations of 2.3 and study the inclusion $M_{1} \subset N_{2}$ whose Jones projection is $p_{I}$.

\section{Proposition 2.4.2.}

(1) The algebra $M_{1}=\left\{p_{I}\right\}^{\prime} \cap N_{2}$ is the subalgebra of $N_{2}$ obtained by cross product of $N_{1}$ by $\delta(I)=N_{0}^{\prime} \cap M_{1}$;

(2) Let $N_{2}^{I}$ be the subalgebra of the points of $N_{2}$ which are fixed under the action of I; then, $M_{1}=h^{-1} N_{2}^{I} h$;

(3) $M_{1}=N_{1} \rtimes \delta(I) \subset N_{2}=N_{1} \rtimes A \subset M_{3}=N_{2} \rtimes I$ is the basic construction. The relative commutant $M_{1}^{\prime} \cap M_{3}$ is $I \cap j_{2}(I)$.

(4) The indices satisfy the following relations:

$$
\left[N_{2} \rtimes I: N_{2}\right]=\left[N_{2}: N_{2}^{I}\right] \text { and }\left[N_{1} \rtimes \delta(I): N_{1}\right]\left[N_{2} \rtimes I: N_{2}\right]=\left[N_{2} \rtimes B: N_{2}\right] .
$$

(5) For any two coideal subalgebras $I_{1}$ and $I_{2}$,

$$
I_{1} \subset I_{2} \quad \Leftrightarrow \quad p_{I_{2}} \leq p_{I_{1}} .
$$

Proof.

(1) The element of $\mathrm{l}(A)$ associated to $M_{1}$ by the Galois correspondence (see 2.2.3) is $\delta(I)$; indeed:

$$
N_{0}^{\prime} \cap M_{1}=N_{0}^{\prime} \cap\left\{p_{I}\right\}^{\prime} \cap N_{2}=\left\{p_{I}\right\}^{\prime} \cap A=\delta(I) .
$$


(2) Let $y=h z h^{-1}$, with $z \in M_{1}$. Then, for $b \in I$,

$$
\begin{aligned}
b \triangleright y & =\tau^{-1} E_{N_{2}}\left(b h z f_{2} h^{-1}\right) & & \tau^{-1} E_{N_{2}}\left(b h z p_{I} f_{2} h^{-1}\right) \\
& =\tau^{-1} E_{N_{2}}\left(b h p_{I} z f_{2} h^{-1}\right) & & \tau^{-1} \tau_{I} E_{N_{2}}\left(b x_{I} h^{-1} z f_{2} h^{-1}\right) \\
& =\tau^{-1} \tau_{I} E_{N_{2}}\left(b e_{I} x_{I} h^{-1} z f_{2} h^{-1}\right) & & \tau^{-1} \tau_{I} E_{N_{2}}\left(\varepsilon_{B}^{t}(b) e_{I} x_{I} h^{-1} z f_{2} h^{-1}\right) \\
& =\tau^{-1} \tau_{I} \varepsilon_{B}^{t}(b) E_{N_{2}}\left(e_{I} x_{I} h^{-1} z f_{2} h^{-1}\right) & & \tau^{-1} \varepsilon_{B}^{t}(b) E_{N_{2}}\left(h p_{I} z f_{2} h^{-1}\right) \\
& =\tau^{-1} \varepsilon_{B}^{t}(b) E_{N_{2}}\left(h z f_{2} h^{-1}\right) & & =\varepsilon_{B}^{t}(b) \triangleright y .
\end{aligned}
$$

Therefore, $M_{1}$ is contained in $h^{-1} N_{2}^{I} h$.

Take reciprocally $y \in h^{-1} N_{2}^{I} h$; then, the following sequence of equality holds:

$$
\begin{aligned}
p_{I} h^{-1} \triangleright h y h^{-1} & =\varepsilon_{B}^{t}\left(p_{I} h^{-1}\right) \triangleright\left(h y h^{-1}\right) \\
\tau^{-1} E_{N_{2}}\left(p_{I} y f_{2}\right) h^{-1} & =\tau^{-1} E_{N_{1}^{\prime} \cap N_{2}}\left(p_{I} f_{2}\right) y h^{-1} \\
\tau^{-1} E_{N_{2}}\left(p_{I} y p_{I} f_{2}\right) & =y \\
\tau^{-1} E_{M_{1}}(y) E_{N_{2}}\left(p_{I} f_{2}\right) & =y \\
E_{M_{1}}(y) & =y
\end{aligned}
$$

Therefore, $y$ belongs to $M_{1}$ and (2) is proved.

(3) The algebra $M_{3}=\left\langle N_{2}, p_{I}\right\rangle$ obtained by basic construction from $M_{1} \subset N_{2}$ is, using (1):

$$
J_{2} M_{1}^{\prime} J_{2}=J_{2}\left(N_{1} \cup \delta(I)\right)^{\prime} J_{2}=J_{2} N_{1}^{\prime} \cap \delta(I)^{\prime} J_{2}=\left(J_{2} N_{1}^{\prime} J_{2}\right) \cap\left(J_{2} \delta(I)^{\prime} J_{2}\right)
$$

On one hand $J_{2} N_{1}^{\prime} J_{2}$ is $N_{3}$, and on the other hand, $\delta(I)^{\prime}$ contains both $N_{2}^{\prime}$ and $\delta(I)^{\prime} \cap B$. Therefore, by [NV00c, 3.3], $M_{3}$ contains both $N_{2}$ and $I$, while being also contained in $N_{2} \rtimes I$. Hence $M_{3}=N_{2} \rtimes I$. Furthermore,

$$
I \cap \tilde{I}=I \cap j_{2}(I)=\left(N_{1}^{\prime} \cap M_{3}\right) \cap\left(M_{1}^{\prime} \cap N_{3}\right)=M_{1}^{\prime} \cap M_{3} .
$$

(4) Follows from (2), (3), and Proposition 2.1.8 of [Jon83.

(5) Y. Watatani shows at the beginning of [Wat96, Part II] that if $M$ and $P$ are two intermediate subfactors of $N_{1} \subset N_{2}$, then $M$ is contained in $P$ if and only if $e_{M}^{N_{2}}$ is dominated by $e_{P}^{N_{2}}$. Let us apply this result to $P=J_{2}\left(N_{2} \rtimes I_{1}\right)^{\prime} J_{2}$ and $M=J_{2}\left(N_{2} \rtimes I_{2}\right)^{\prime} J_{2}$, where $I_{1}$ and $I_{2}$ are two coideal subalgebras; then, $p_{I_{2}} \leq p_{I_{1}}$ is equivalent to $M \subset P$ and therefore to $I_{1} \subset I_{2}$ since $\delta$ is an antiisomorphism.

2.5. The principal graph of an intermediate inclusion. The principal graph of an inclusion is obtained from the Bratelli diagram of the tower of relative commutants or from the equivalence classes of simple bimodules (see [GdlHJ89], and [JS97]). It is an invariant of the inclusion.

By [NV00c, 5.9], the principal graph of the inclusion $N_{2} \subset N_{2} \rtimes I$ is the connected component of the Bratteli diagram of $\delta(I) \subset A$ containing the trivial representation of $A$. All the principal graphs we obtained in our examples are gathered in Section 4 .

\subsection{Depth 2 intermediate inclusions.}

2.6.1. $C^{*}$-quantum subgroupoid. A coideal subalgebra $I$ is a $C^{*}$-quantum subgroupoid of $B$ if it is stabilized by the coproduct and antipode of $B$; then, it becomes a $C^{*}$-quantum groupoid for the structure induced from $B$. The following proposition provides some equivalent characterizations.

Proposition 2.6.1. For I a coideal subalgebra of $B$, the following are equivalent:

(i) $I$ is a $C^{*}$-quantum subgroupoid of $B$;

(ii) $I$ is stabilized by the antipode $S$; 
(iii) $I$ is stabilized by the antiautomorphism $j_{2}$;

(iv) $I$ is stabilized by $\Delta: \Delta(I) \subset I \otimes I$;

(v) $\Delta^{\varsigma}\left(p_{I}\right)=\left(h^{-1} j_{2}(h) \otimes 1\right) \Delta\left(p_{I}\right)\left(h^{-1} j_{2}(h) \otimes 1\right)$.

Proof. By definition, (i) is equivalent to (ii) and (iv) together.

(ii) $\Longleftrightarrow$ (iii): It is sufficient to remark that if $I$ is stabilized by $S$ or $j_{2}$, then $j_{2}(h)$ belongs to $I$. It remains to use the relation between $S, h$ and $j_{2}$ (see 2.1.2).

(iii) $\Longrightarrow(\mathrm{v})$ : Assume that $I$ is stabilized by $j_{2}$. Then $\left\{j_{2}\left(c_{t}^{*}\right) \mid t \in T\right\}$ is a family of normalized matrix units of $I$; using it, one can write the coproduct of $p_{I}$ and check the claimed relation between $\Delta\left(p_{I}\right)$ et $\Delta^{\sigma}\left(p_{I}\right)$.

$(\mathrm{v}) \Longrightarrow$ (iii): Assume that $\Delta^{\sigma}\left(p_{I}\right)=\left(h^{-1} j_{2}(h) \otimes 1\right) \Delta\left(p_{I}\right)\left(h^{-1} j_{2}(h) \otimes 1\right)$. Then the left and right legs of $\Delta\left(p_{I}\right)$ span the same subspace, which is $I=j_{2}(I)$.

(ii) $\Longrightarrow$ (iv):

$$
\Delta(I)=\left(S^{-1} \otimes S^{-1}\right) \Delta^{\sigma}(S(I)) \subset S^{-1}(I) \otimes I=I \otimes I,
$$

(iv) $\Longrightarrow$ (iii): Assume $\Delta(I) \subset I \otimes I$. Then, $\Delta\left(p_{I}\right)$ is in $I \otimes I$, and by Proposition 2.3.2, $I$ is stabilized by $j_{2}$.

2.6.2. $C^{*}$-quantum subgroupoid and intermediate inclusions. From 2.4 .2 (3) and 2.6.1, a coideal subalgebra $I$ is a $C^{*}$-quantum subgroupoid of $B$, if and only if $I=M_{1}^{\prime} \cap M_{3}$. If $I$ is a $C^{*}$-quantum groupoid, the inclusion $N_{2} \subset N_{2} \rtimes I$ is of depth 2. In 2.8.3, we will show that the reciprocal holds as soon as the inclusions are irreducible, while the following example shows that it can fail without the irreducibility condition.

2.6.3. An example. As in [NV02, 2.7] and [Dav05, 5], we consider the Jones subfactor of index $4 \cos ^{2} \frac{\pi}{5}$ in $R$. We denote by $P_{0} \subset P_{1}$ this inclusion whose principal graph is $A_{4}$ and write

$$
P_{0} \subset P_{1} \subset P_{2} \subset P_{3} \subset P_{4} \subset P_{5} \subset P_{6} \subset P_{7} \subset P_{8} \subset P_{9}
$$

the tower obtained by basic construction. The relative commutants are minimals; they are the Temperley-Lieb algebras. From [NV00c, 4.1], the inclusions $P_{0} \subset P_{2}$ and $P_{0} \subset P_{3}$ are of depth 2. Consider $P_{8}$ as intermediate subfactor of $P_{6} \subset P_{9}$; it is obtained by cross product of $P_{6}$ by a coideal subalgebra $I=P_{3}^{\prime} \cap P_{8}$ of $P_{3}^{\prime} \cap P_{9}$. The algebra $P_{3}^{\prime} \cap P_{9}$ is endowed with a $C^{*}$-quantum groupoid structure since the inclusion $P_{0} \subset P_{3}$ is of depth 2. On the other hand, since the inclusion $P_{0} \subset P_{2}$ is of depth 2, this is also the case for $P_{6} \subset P_{8}$. However, by dimension count, $P_{4}^{\prime} \cap P_{8}$ is strictly contained in $I$.

2.7. The $C^{*}$-quantum groupoids of dimension 8 . The smallest non trivial $C^{*}$-quantum groupoids are of dimension 8. They are described in [NV00a, 3.1] and in [NV00b, 7.2]. L. Vainerman pointed to us that, since their representation categories are the same as for $\mathbb{Z}_{2}$, their intermediate subfactors lattices are trivial. Together with the Kac-Paljutkin algebra $K P$, this covers all the non trivial $C^{*}$-quantum groupoids of dimension 8.

2.8. Special case of irreducible inclusions. In this article, we concentrate on Kac algebras, and therefore on irreducible inclusions of finite index. In this case, any intermediate inclusion is of integral index (see [Bis94a]); in fact, as we showed in 2.2.4, the index of $N_{2} \subset N_{2} \rtimes I$ is the dimension of $I$. Furthermore, the lattice of intermediate subfactors is finite (see [Wat96]). 
2.8.1. Kac algebras and $C^{*}$-Hopf algebras. This is a summary of [ES92, 6.6] which relates Kac algebras and $C^{*}$-Hopf algebras. Let $B$ be a $C^{*}$-algebra of finite dimension 5 . Set $B=\oplus_{j} M_{n_{j}}(\mathbb{C}) q_{j}$ and denote by $T r_{j}$ the canonical trace 6 on the factor $M_{n_{j}}(\mathbb{C}) q_{j}$. The normalized canonical trace of $B$ is given by the formula $\operatorname{tr}(x)=\frac{1}{\operatorname{dim} B} \sum_{j} n_{j} \operatorname{Tr}_{j}\left(x q_{j}\right)$.

If $(B, \Delta, \varepsilon, S)$ is a $C^{*}$-Hopf algebra, then $(B, \Delta, S, t r)$ is a Kac algebra. Reciprocally, if $(B, \Delta, S, t r)$ is a Kac algebra, then, from [ES92, 6.3.5], there exist a projection $p$ of dimension 1 in the center of $B$ such that $\varepsilon$, defined by $\varepsilon(x)=\operatorname{tr}(x p)$, is the counit of $B$, and $(B, \Delta, \varepsilon, S)$ is a $C^{*}$-Hopf algebra.

The coinvolution, or antipode, of a Kac algebra constructed from a depth 2 irreducible inclusion coincides with $j_{2}$ (voir [Dav96]). From [Kas95, III.3.2], the antipode of a $C^{*}$ algèbre de Hopf is uniquely determined by the rest of the structure. Also, in the examples we consider, the coinvolution is not deformed. Therefore, we often skip in the sequel the obvious checks on the coinvolution.

2.8.2. The following proposition summarizes the properties of coideal subalgebras and their Jones projections in the irreducible case:

Proposition 2.8.2. Assume that the inclusion $N_{0} \subset N_{1}$ is irreducible, and consider a coideal subalgebra $I$ in $\mathrm{l}(B)$. Then, $I$ is connected, and

(1) $\tau_{I}^{-1}=\operatorname{tr}\left(p_{I}\right)^{-1}=\left[N_{2} \rtimes I: N_{2}\right]=\operatorname{dim} I$, and $\operatorname{dim} I$ divides $\operatorname{dim} B$.

(2) The Jones projection $p_{I}$ dominates $f_{2}$, belongs to the center of $I$, and satisfies:

$$
I p_{I}=\mathbb{C} p_{I} \quad \text { and } \quad \varepsilon(b)=\operatorname{dim} I \operatorname{tr}\left(p_{I} b\right), \text { for all } b \in I .
$$

(3) The coideal subalgebra $I$ is the subspace spanned by the right legs of $p_{I}$, and

$$
\Delta\left(p_{I}\right)=(\operatorname{dim} I)^{-1} \sum_{t \in T} S\left(c_{t}^{*}\right) \otimes c_{t} .
$$

(4) $\Delta\left(p_{I}\right)\left(1 \otimes p_{I}\right)=\left(p_{I} \otimes p_{I}\right)$.

Proof. (1) follows from corollary 2.2.4 and 2.4.2 (4).

(2) The counital algebras are trivial, the element $h$ is 1 , the projections $e_{I}$ and $p_{I}$ coincide with $(\operatorname{dim} I)^{-1} x_{I}$. Take $y \in I$. From [NV00c, 3.7] (see also 2.3.4), one has $y p_{I}=\varepsilon(y) p_{I}$, and it follows successively that $p_{I} y^{*}=\overline{\varepsilon(y)} p_{I}$, and $p_{I} y=\overline{\varepsilon\left(y^{*}\right)} p_{I}=\varepsilon(y) p_{I}$. Therefore $p_{I}$ is in the center of $I$.

(3) follows from 2.3.2.

(4) Since $I p_{I}$ is of dimension 1 , one can set $c_{t_{0}}=\tau_{I}^{-1 / 2} p_{I}$ for some $t_{0} \in T$, and $c_{t} p_{I}=0$ for all $t \in T \backslash\left\{t_{0}\right\}$. The desired equation follows easily.

An equivalent statement of Proposition 2.8.2 (4) can be found in BBS99; namely, in their setting, the image of $p_{I}$ in $L^{2}(B, t r)$ is a presubgroup.

The following remark is useful in practice when searching for coideal subalgebras.

Remark 2.8.2. Let $p$ be a projection of trace $1 / k$, for some divisor $k$ of $\operatorname{dim} B$, which dominates $f_{2}$ and generates a coideal subalgebra $I$ of dimension $k$. Then, $p$ is the Jones projection $p_{I}$ of $I$; indeed, $p$ dominates $p_{I}$ and has the same trace.

\footnotetext{
${ }^{5}$ Recall that any $C^{*}$-algebra of finite dimension is semi-simple.

${ }^{6} \operatorname{Tr}_{j}\left(q_{j}\right)=n_{j}$.
} 
2.8.3. Intermediate inclusions of depth 2 . The following proposition characterize the depth 2 intermediate inclusions in the case of irreducible inclusions.

Proposition 2.8.3. Let I be a coideal subalgebra of $B$. Then, the following are equivalent:

(i) The inclusion $N_{2} \subset N_{2} \rtimes I$ is of depth 2 ;

(ii) $I$ is a Kac subalgebra of $B$;

(iii) the coproduct of $p_{I}$ is symmetric;

(iv) $I=M_{1}^{\prime} \cap M_{3}$.

Proof. In the irreducible case, Proposition 2.6 .1 implies that $(i i) \Leftrightarrow(i i i) \Leftrightarrow(i v)$; indeed, by 2.4 .2 (3), (iv) is equivalent to $I=j_{2}(I)$.

Furthermore (ii) implies (i). Assume reciprocally (i): $N_{2} \subset N_{2} \rtimes I$ of depth 2. Then $M_{1}^{\prime} \cap M_{3}$ is a Kac algebra of dimension $\left[M_{3}: N_{2}\right]=\operatorname{dim} I$ contained in $I$; therefore $M_{1}^{\prime} \cap M_{3}=I \cap j_{2}(I)$ coincides with $I$, that is (iv).

2.8.4. Special case of group algebras. GdlHJ89, 4.7], [JS97, A.4] et [HS96, 2]

We now describe the special case of group algebras and their duals.

Let $G$ be a finite group. We denote by $\left(\mathbb{C}[G], \Delta_{s}\right)$ the symmetric Kac algebra of the group, and by $L^{\infty}(G)$ the commutative Kac algebra of complex valued functions on the group. Those two algebras are in duality.

If $G$ acts outerly on a $I I_{1}$ factor $N$, then $N^{G} \subset N \subset N \rtimes G \subset(N \rtimes G) \rtimes L^{\infty}(G)$ is the basic construction. The principal graph of $N^{G} \subset N$ (resp. $N \subset N \rtimes G$ ) is the Bratelli diagram of $\mathbb{C} \subset \mathbb{C} G$ (resp. $\mathbb{C} \subset L^{\infty}(G)$ ).

The intermediate subfactors of $N \subset N \rtimes G$ are of the form $N \rtimes H$, where $H$ is a subgroup of $G$; indeed, since the coproduct $\Delta_{s}$ is symmetric, any coideal subalgebra of $\mathbb{C}[G]$ is a symmetric Kac subalgebra, hence the algebra of a subgroup $H$ of $G$. The Jones projection of $\mathbb{C}[H]$ is $\frac{1}{|H|} \sum_{h \in H} h$. Remark that $H$ is normal if and only if $p_{H}$ belongs to the center of $\mathbb{C}[G]$.

If $H$ is commutative, from [Vai98, page 714], the matrix units of $\mathbb{C}[H]$ are the projections $P_{h}=\frac{1}{|H|} \sum_{g \in H}\langle h, g\rangle \lambda(g)$, for $h \in \widehat{H}$, with standard coproduct:

$$
\Delta_{s}\left(P_{h}\right)=\sum_{g \in \widehat{H}} P_{g} \otimes P_{g^{-1} h}
$$

More precisely, for the Jones projection, one get using Proposition 2.8 .2 (3):

$$
\Delta_{s}\left(\frac{1}{|H|} \sum_{g \in H} \lambda(g)\right)=\sum_{h \in \widehat{H}} S\left(P_{h}\right) \otimes P_{h} .
$$

The intermediate subfactors of $N \rtimes G \subset(N \rtimes G) \rtimes L^{\infty}(G)$ are of the form $(N \rtimes G) \rtimes I$, where $I$ is a coideal subalgebra of $L^{\infty}(G)$; they are obtained by basic construction from the inclusion $N \rtimes H \subset N \rtimes G$, where $\delta(I)=\mathbb{C}[H]$. The coideal subalgebra $I$ is then the algebra $L^{\infty}(G / H)$ of functions which are constant on the right $H$-cosets of $G$. Indeed $L^{\infty}(G / H)$ is an involutive subalgebra, and by definition of the coproduct $\Delta(f)(s, t)=f(s t)$, it is a coideal subalgebra which is furthermore a Kac subalgebra if and only if $H$ is normal. Its Jones projection is $\sum_{h \in H} \chi_{h}$, where $\chi_{h}$ is the characteristic function of $h$.

\footnotetext{
${ }^{7}$ Recall that any finite dimensional symmetric (resp. commutative) Kac algebra is the algebra of a group (resp. the algebra of functions on a group) ES92
} 
2.8.5. Normal coideal subalgebras. Generalizing from group algebras and their dual (see 2.8.4), we define normal coideal subalgebras as follow (see also [KP66] and [Nik98, 2.2]).

Definition 2.8.5. A coideal subalgebra $I$ of $B$ is normal if $I$ is a Kac subalgebra of $B$, and $p_{I}$ belongs to the center of $B$.

The following characterization of normal coideal subalgebras results from 2.8.3 and 2.5.

Proposition 2.8.5. A coideal subalgebra $I$ of $B$ is normal if and only if the inclusions $N_{1} \subset M_{1}$ and $M_{1} \subset N_{2}$ are of depth 2 if and only if $p_{I}$ belongs to the center of $B$ and the coproduct of $p_{I}$ is symmetric.

Example 2.8.5. In $K D(2 m)$, the coideal subalgebras $K_{j}$, for $j=1, \ldots, 4$, are normal (see 7).

2.8.6. Intrinsic group. The intrinsic group $G(K)$ of a Kac algebra $K$ is the subset of invertible elements $x$ of $K$ satisfying $\Delta(x)=x \otimes x$ [ES92, 1.2.2]. It is in correspondence with the subset of characters of the dual Kac algebra of $K$ [ES92, 3.6.10].

The group algebra $\mathbb{C}[G(K)]$ of $G(K)$ is a Kac subalgebra of $K$ which contains any symmetric Kac subalgebra of $K$. In particular, since any inclusion of index 2 results from a cross product by $\mathbb{Z}_{2}$, any coideal subalgebra of dimension 2 is contained in $\mathbb{C}[G(K)]$. 


\section{The KaC-Paluutkin algebra}

In this section, we study the Kac-Paljutkin algebra $K P$ [KP66]. This is the unique non trivial Kac algebra of dimension 8, making it the smallest non trivial Kac algebra. The study of the lattice of coideal subalgebras of $K P$, which does not require heavy calculations, gives a simple illustration of the results of 2. Furthermore, $K P$ will often occur as Kac subalgebra in the sequel.

3.1. The Kac-Paljutkin algebra KP. We use the description and notations of [EV96]. The Kac-Paljutkin algebra is the $C^{*}$-algebra

$$
K P=\mathbb{C} e_{1} \oplus \mathbb{C} e_{2} \oplus \mathbb{C} e_{3} \oplus \mathbb{C} e_{4} \oplus M_{2}(\mathbb{C}),
$$

endowed with its unique non trivial Kac algebra structure. The matrix units of the factor $M_{2}(\mathbb{C})$ are denoted by $e_{i, j}(i=1,2$ and $j=1,2)$. From 2.8.1, the trace is given by: $\forall i=1,2,3,4 \operatorname{tr}\left(e_{i}\right)=\frac{1}{8}$ and $\operatorname{tr}\left(e_{1,1}\right)=\operatorname{tr}\left(e_{2,2}\right)=\frac{1}{4}$.

The Kac algebra structure can be constructed by twisting the group algebra of

$$
G=\{1, a, b, a b=b a, c, a c=c b, b c=c a, a b c=c a b\} .
$$

We denote by $\lambda$ the left regular representation of $G$ :

$$
\begin{aligned}
& \lambda(a)=e_{1}-e_{2}+e_{3}-e_{4}-e_{1,1}+e_{2,2} \\
& \lambda(b)=e_{1}-e_{2}+e_{3}-e_{4}+e_{1,1}-e_{2,2} \\
& \lambda(c)=e_{1}+e_{2}-e_{3}-e_{4}+e_{1,2}+e_{2,1}
\end{aligned}
$$

and by $\Delta$ the coproduct of $K P$ given explicitly in [EV96].

3.2. The coideal subalgebras of $K P$. We now determine $l(K P)$, using the results of 2.8.2. Note first that the Jones projection of the non trivial coideal subalgebras are projections of trace either $\frac{1}{2}$ or $\frac{1}{4}$ which dominate $e_{1}$, the support of the counit of $K P$ hence the Jones projection of $K P$.

3.2.1. Since the coproduct is not deformed on $\mathbb{C}\left[\left(\mathbb{Z}_{2}\right)^{2}\right], 1(K P)$ contains the Kac subalgebras:

- $J_{0}=\mathbb{C}\left(e_{1}+e_{3}\right) \oplus \mathbb{C}\left(e_{2}+e_{4}\right) \oplus \mathbb{C} e_{1,1} \oplus \mathbb{C} e_{2,2}$ spanned by $1, \lambda(a), \lambda(b)$, and $\lambda(a b)$;

- $I_{0}=\mathbb{C}\left(e_{1}+e_{2}+e_{3}+e_{4}\right) \oplus \mathbb{C}\left(e_{1,1}+e_{2,2}\right)$ spanned by 1 and $\lambda(a b)$;

- $I_{1}=\mathbb{C}\left(e_{1}+e_{3}+e_{1,1}\right) \oplus \mathbb{C}\left(e_{2}+e_{4}+e_{2,2}\right)$ spanned by 1 and $\lambda(b)$;

- $I_{2}=\mathbb{C}\left(e_{1}+e_{3}+e_{2,2}\right) \oplus \mathbb{C}\left(e_{2}+e_{4}+e_{1,1}\right)$ spanned by 1 and $\lambda(a)$.

The Jones projections of those subalgebras are therefore respectively

$$
p_{0}=e_{1}+e_{3}, \quad p_{0}^{\prime}=e_{1}+e_{2}+e_{3}+e_{4}, \quad p_{1}^{\prime}=e_{1}+e_{3}+e_{1,1}, \quad \text { and } \quad p_{2}^{\prime}=e_{1}+e_{3}+e_{2,2} .
$$

Since the intrinsic group of $K P$ is $J_{0}$, by 2.8.6, $I_{0}, I_{1}$, and $I_{2}$ are the only dimension 2 coideal subalgebras.

3.2.2. Since the Kac-Paljutkin algebra is self-dual, $1(K P)$ is also self-dual. We therefore can obtain the other elements of $1(K P)$ by using the antiisomorphism $\delta$. Since $J_{0}$ is the group algebra of $\left(\mathbb{Z}_{2}\right)^{2}$, the inclusion $N_{2} \subset N_{2} \rtimes J_{0}$ is of index 4 , and its principal graph is $D_{4}^{(1)}$ (see 4.3); from 2.5, this graph is the connected component of the Bratteli diagram of $\delta\left(J_{0}\right) \subset K P$ which contains $\mathbb{C} e_{1}$. Therefore, the Jones projection of $\delta\left(J_{0}\right)$ is $e_{1}+e_{2}+e_{3}+e_{4}$, and $\delta\left(J_{0}\right)$ is $I_{0}$. 
3.2.3. Since $\delta$ is a lattice antiisomorphism, $\delta\left(I_{1}\right)$ and $\delta\left(I_{2}\right)$ contain $I_{0}=\delta\left(J_{0}\right)$; furthermore, their Jones projection (which dominate $e_{1}$ ) are of trace $\frac{1}{4}$. Only two remaining projections are possible: $p_{2}=e_{1}+e_{2}$ et $p_{4}=e_{1}+e_{4}$.

Denote by $q_{k}$ the projection $\frac{1}{2}\left(\begin{array}{cc}1 & \mathrm{e}^{-\mathrm{i} k \pi / 4} \\ \mathrm{e}^{\mathrm{i} k / / 4} & 1\end{array}\right)$ of the factor $M_{2}(\mathbb{C})$. We describe the coideal subalgebra $J_{2}$ generated by $p_{2}$. From the equality

$$
\begin{aligned}
\Delta\left(p_{2}\right)=\left(e_{1}+e_{2}\right) & \otimes\left(e_{1}+e_{2}\right)+\left(e_{3}+e_{4}\right) \otimes\left(e_{3}+e_{4}\right) \\
+ & \frac{1}{2}\left(e_{1,1}+e_{2,2}\right) \otimes\left(e_{1,1}+e_{2,2}\right)+\frac{1}{2}\left(e_{1,2}-i e_{2,1}\right) \otimes\left(e_{1,2}+i e_{2,1}\right),
\end{aligned}
$$

we deduce that $J_{2}$ contains $e_{3}+e_{4}, e_{1,1}+e_{2,2}$ (which we already know since $J_{2}$ contains $p_{2}$ and $\left.I_{0}\right)$ and $u=e_{1,2}+i e_{2,1}$. It therefore contains the symmetry $s=\mathrm{e}^{-\mathrm{i} \pi / 4} \mathrm{u}$, and the projection $q_{1}=\frac{1}{2}\left(e_{1,1}+e_{2,2}+s\right)$. More precisely:

$$
\Delta\left(p_{2}\right)=\left(e_{1}+e_{2}\right) \otimes\left(e_{1}+e_{2}\right)+\left(e_{3}+e_{4}\right) \otimes\left(e_{3}+e_{4}\right)+q_{7} \otimes q_{1}+q_{3} \otimes q_{5}
$$

It follows that:

$$
J_{2}=\mathbb{C}\left(e_{1}+e_{2}\right) \oplus \mathbb{C}\left(e_{3}+e_{4}\right) \oplus \mathbb{C} q_{1} \oplus \mathbb{C} q_{5} .
$$

Similarly, $J_{4}$ can be described as follow:

$$
J_{4}=\mathbb{C}\left(e_{1}+e_{4}\right) \oplus \mathbb{C}\left(e_{2}+e_{3}\right) \oplus \mathbb{C} q_{3} \oplus \mathbb{C} q_{7} .
$$

From 2.5, the principal graph of the inclusions $N_{2} \subset N_{2} \rtimes J_{2}$ and $N_{2} \subset N_{2} \rtimes J_{4}$ is the connected component of the Bratteli diagram of $I_{j} \subset K P, j=1,2$ containing $\mathbb{C} e_{1}$; in both case, it is $D_{6}^{(1)}$ (see 4.3).

3.3. The lattice $1(K P)$. We have now completed the construction of $1(K P)$.

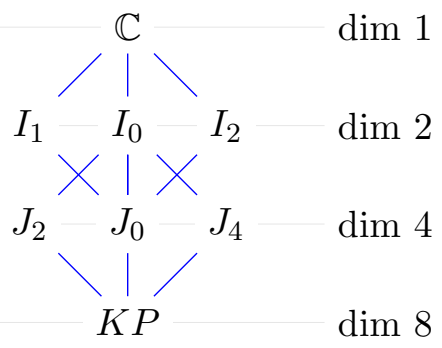

Figure 1. The lattice of coideal subalgebras of $K P$

Proposition 3.3. The lattice of coideal subalgebras of the Kac-Paljutkin algebra is as given in Figure 1 .

Proof. The Jones projections of the coideal subalgebras of dimension 4 are necessarily of the form $e_{1}+e_{j}$ with $j=2,3$ or 4 , and the coideal subalgebras of dimension 2 are all contained in $J_{0}$.

Thanks to the Galois correspondence, we can deduce the non trivial intermediate subfactors of the inclusion $N_{2} \subset N_{2} \rtimes K P$ :

- Three factors $N_{2} \rtimes I_{i}, i=0,1,2$, isomorphic to $N_{2} \rtimes \mathbb{Z}_{2}$;

- The factor $N_{2} \rtimes J_{0}=N_{2} \rtimes\left(\mathbb{Z}_{2}\right)^{2}$ with principal graph $D_{4}^{(1)}$;

- Two factors $N_{2} \rtimes J_{i}, i=1,2$, with principal graph $D_{6}^{(1)}$. 
3.4. Realization of $K P$ by composition of subfactors. In [IK02, VIII], Izumi and Kosaki consider an inclusion of principal graph $D_{6}^{(1)}$, from which they construct a depth 2 inclusion isomorphic to $R \subset R \rtimes K P$. We can now describe explicitly all the ingredients of their construction (see also [Pop90]).

Proposition 3.4. The inclusion $N_{2} \subset N_{2} \rtimes J_{2}$, which is of principal graph $D_{6}^{(1)}$, can be put under the form $M^{\left(\alpha, \mathbb{Z}_{2}\right)} \subset M \rtimes_{\beta} \mathbb{Z}_{2}$ as follows. Set $M=N_{2} \rtimes I_{0}, v=\left(e_{1}+e_{2}\right)-\left(e_{3}+e_{4}\right)+s$ and $\beta=\operatorname{Ad} v$. Let $\alpha$ be the automorphism of $M$ which fixes $N_{2}$ and changes $\lambda(a b)$ into its opposite (this is the dual action of $\mathbb{Z}_{2}$ ). Then, $\alpha$ and $\beta$ are involutive automorphisms of $M$ such that the outer period of $\beta \alpha$ is 4 , and

$$
(\beta \alpha)^{4}=\operatorname{Ad} \lambda(a b), \quad \beta \alpha(\lambda(a b))=-\lambda(a b), \quad M^{\alpha}=N_{2}, \quad \text { and } \quad N_{2} \rtimes J_{2}=M \rtimes_{\beta} \mathbb{Z}_{2} .
$$

Proof. As in 2.1.2 (see [Dav05]), we identify $N_{3}$ and $N_{2} \rtimes K P$. Therefore, for all $x \in N_{2}$, one has $\beta(x)=v x v^{*}=\left(v_{(1)} \triangleright x\right) v_{(2)} v^{*}$. From $\Delta(v)=v \otimes\left[\left(e_{1}+e_{2}\right)-\left(e_{3}+e_{4}\right)\right]+v^{\prime} \otimes s$, (with $\left.v^{\prime}=\left(e_{1}+e_{4}\right)-\left(e_{2}+e_{3}\right)+\mathrm{e}^{\mathrm{i} \pi / 4} e_{1,2}+\mathrm{e}^{-\mathrm{i} \pi / 4} e_{2,1}\right)$, we get, for $x \in N_{2}$,

$$
\beta(x)=8\left[E_{N_{2}}\left(v x e_{1}\right)\left(e_{1}+e_{2}+e_{3}+e_{4}\right)+E_{N_{2}}\left(v^{\prime} x e_{1}\right)\left(e_{1,1}+e_{2,2}\right)\right],
$$

and deduce that $\beta$ normalizes $M$; by a straightforward calculation, the two automorphisms satisfy the claimed relations. Then, $N_{2} \rtimes J_{2}$ is indeed the cross product of $M$ by $\beta$, since $\lambda(a b)$ and $v$ generate the subalgebra $J_{2}$. 


\section{Principal GRAPHS OBtained in OUR EXAMPLES}

Using 2.5, the examples we study in the sequel yield several families of non trivial principal graphs of inclusions which we collect and name here.

4.1. Graphs $D_{2 n+1} / \mathbb{Z}_{2}$. In 6.2, 6.7, 8.4.2, and 7.1.3, we obtain the graphs $D_{2 n+1} / \mathbb{Z}_{2}$, where $n$ is the number of vertices in the spine of the graph (altogether $n+4$ vertices). The inclusions are then of index $2 n+1$. The graph $D_{11} / \mathbb{Z}_{2}$ is depicted in Figure 2 ,

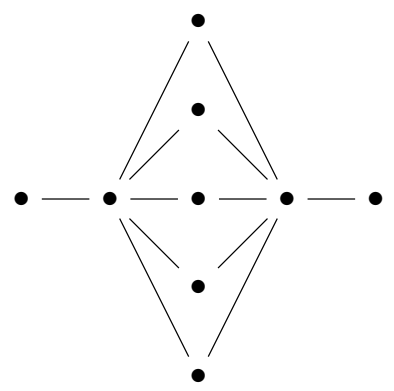

Figure 2. The graph $D_{11} / \mathbb{Z}_{2}$

Those graphs are the principal graphs of the inclusions $R \rtimes \mathbb{Z}_{2} \subset R \rtimes D_{2 n+1}$ dBG91. Furthermore, any inclusion admitting such a principal graph is of the form $R \rtimes H \subset R \rtimes G$ where $G$ is a group of order $2(2 n+1)$ obtained by semi-direct product of an abelian group and $H \equiv \mathbb{Z}_{2}[$ HS96].

4.2. Graphs $D_{2 n+2} / \mathbb{Z}_{2}$. In 6.2 and 7.1 .3 , we obtain the graphs $D_{2 n+2} / \mathbb{Z}_{2}$, where $n$ is the number of vertices in the spine (altogether $n+6$ vertices). The inclusion is then of index $2(n+1)$. The graph $D_{12} / \mathbb{Z}_{2}$ is depicted in Figure 3. Those graphs are the principal graphs of the inclusions $R \rtimes \mathbb{Z}_{2} \subset R \rtimes D_{2 n+2}$ dBG91].

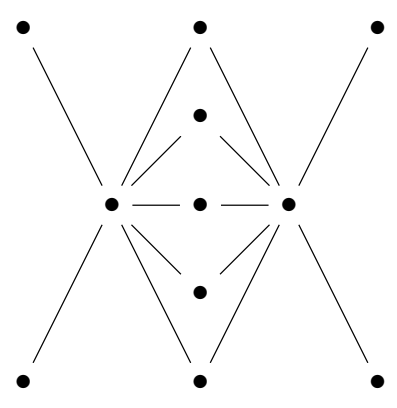

Figure 3 . The graph $D_{12} / \mathbb{Z}_{2}$

4.3. Graphs $D_{n}^{(1)}$. We obtain the graphs $D_{n}^{(1)}$, with $n+1$ vertices, for subfactors of index 4. These graphs $D_{n}^{(1)}$ were readily obtained in Pop90. The graph $D_{6}^{(1)}$ (Figure 4) is the principal graph of two intermediate subfactors of $R \subset R \rtimes K P$ (see 3). The graph $D_{8}^{(1)}$ is the principal graph of two intermediate subfactors of $R \subset R \rtimes K D(3)$ (see 6.9.1). The graph $D_{10}^{(1)}$ is the principal graph of $R \rtimes \delta\left(K_{2 k}\right)$ (with $k \in\{1, \ldots, 4\}$ ) intermediate in $R \rtimes \widehat{K D(4)}$ (see 7.6.3). The graphs $D_{n}^{(1)}$ also occur as principal graphs of $R \rtimes J_{k}$ (with $k \in\{1, \ldots, m\}$ ) intermediate in $R \rtimes K D(2 n+1)$ (see 6.7) and of $R \rtimes J_{k}$ (with $k \in\{1, \ldots, m\}$ ) intermediate in $R \rtimes K Q(2 n+1)$ (see 8.4.2). 


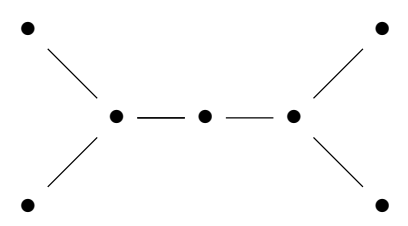

FiguRE 4. The graph $D_{6}^{(1)}$

4.4. Graphs $Q B_{n}$. In 7.1.3, we obtain a family of graphs which we denote $Q B_{n}$. They have $4 n+5$ vertices; $n$ of type $\star$ in each of the three groups, and $n-1$ of type $\square$. The inclusion is then of index $8 n$. The graph $Q B_{2}$ is depicted in Figure 5 ,

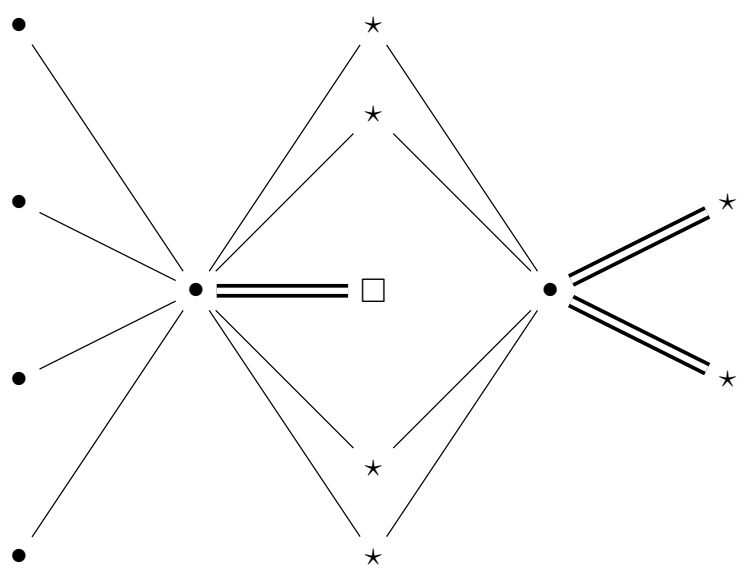

FiguRE 5. The graph $Q B_{2}$

4.5. Graphs $D B_{n}$. In 7.1.3, we find a family of graphs which we denote by $D B_{n}$. They have $4 n+7$ vertices; $n$ of type $\star$ in each of the three groups, and $n+1$ of type $\square$. The inclusion is then of index $8 n+4$. The graph $D B_{2}$ is depicted in Figure 6 .

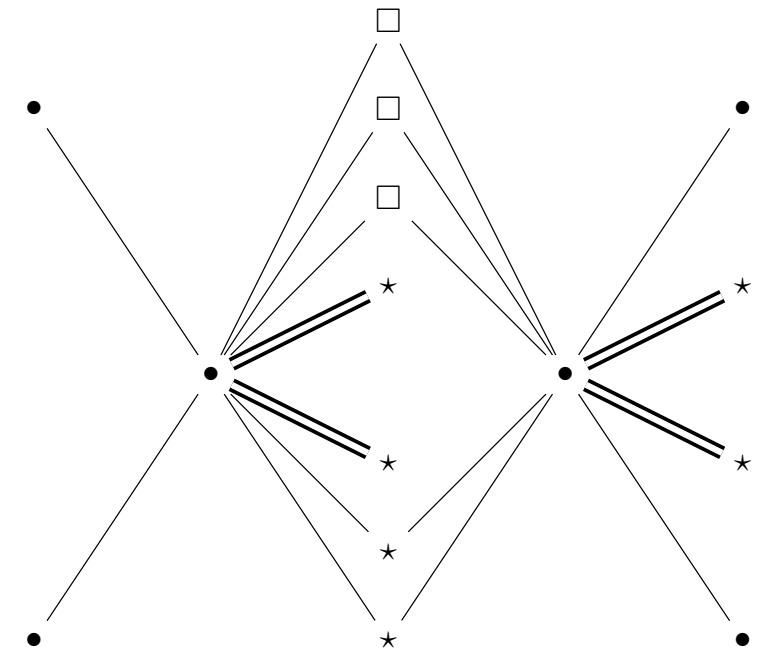

Figure 6. The graph $D B_{2}$ 


\section{The Kac Algebras $K D(n)$ And twisted group Algebras}

In this section, we recall the definition of the family of Kac algebras $(K D(n))_{n}$, describe completely $K D(2)$ (5.3), determine the automorphism group of $K D(n)$, and obtain some general results on the lattice of their coideal subalgebras. The rest of the structure depends much on the parity of $n$, and are further investigated in 6 for $n$ odd and in 7 and 9 for $n$ even.

Along the way, we build some general tools for manipulating twisted group algebras: isomorphism computations (Algorithm [5.5.8) and proofs (Proposition 5.5.3), coideal subalgebras induced by the group (Theorem 5.4.1), efficient characterization of the algebra of the intrinsic group (Corollary [5.4.1). Those tools will be reused extensively in the later sections.

5.1. Twisted group algebras. In [EV96] and Vai98, M. Enock and L. Vainerman construct non trivial finite dimensional Kac algebras by twisting the coproduct of a group algebra by a 2-(pseudo)-cocycle $\Omega$. More precisely, $\Omega$ is obtained from a 2-(pseudo)-cocycle $\omega$ defined on the dual $\hat{H}$ of a commutative subgroup $H$ of $G$. We refer to those articles for the details of the construction.

For example, the Kac-Paljutkin algebra $K P$ studied in 3.3 can be constructed this way (see EV96]). Similarly, the Kac algebras $K D(n)$ (resp. $K Q(n))$ of dimension $4 n$ are obtained in [Vai98, 6.6] by twisting of the algebra of the dihedral group $\mathbb{C}\left[D_{2 n}\right]$ (resp. of the quaternion group).

In Vai98, end of page 714], L. Vainerman gives a condition on the cocycle $\omega$ (to be counital) for $\Omega$ itself to be counital (see definition in Vai98, Lemma 6.2]). Then, the coinvolution exists in the twisted algebra and the counit is well defined, and in fact left unchanged [Vai98, lemma 6.2]. This condition is always satisfied in our examples.

5.2. Notations. Let $n$ be a positive integer, and $D_{2 n}=\left\langle a, b \mid a^{2 n}=1, b^{2}=1, b a=a^{-1} b\right\rangle$ be the dihedral group of order $4 n$. It contains a natural commutative subgroup of order 4: $H=\left\langle a^{n}, b\right\rangle$. We denote by $K D(n)$ the Kac algebra constructed in [Vai98, 6.6] by twisting the group algebra $\mathbb{C}\left[D_{2 n}\right]$ along a 2-cocycle of $H$. For notational convenience we include $K D(1)$ in the definition, even though the twisting is trivial there since $H=D_{2}$. We refer to [Vai98, 6.6] for the full construction, and just recall here the properties that we need. Some further formulas are listed in Appendix A

The algebra structure and the trace are left unchanged; the block matrix structure of $K D(n)$ is given by:

$$
K D(n)=\mathbb{C} e_{1} \oplus \mathbb{C} e_{2} \oplus \mathbb{C} e_{3} \oplus \mathbb{C} e_{4} \oplus \bigoplus_{k=1}^{n-1} K D(n)^{k},
$$

where $K D(n)^{k}$ is a factor $M_{2}(\mathbb{C})$ whose matrix units we denote by $e_{i, j}^{k}(i=1,2$ and $j=1,2)$. From 2.8.1, the trace on $K D(n)$ is given by: $\operatorname{tr}\left(e_{i}\right)=1 / 4 n$ and $\operatorname{tr}\left(e_{j, j}^{k}\right)=1 / 2 n$.

We use the following matrix units in $K D(n)^{k}$ :

$$
r_{1,1}^{k}=\frac{1}{2}\left(\begin{array}{cc}
1 & -\mathrm{i} \\
\mathrm{i} & 1
\end{array}\right), r_{1,2}^{k}=\frac{1}{2}\left(\begin{array}{cc}
\mathrm{i} & -1 \\
-1 & -\mathrm{i}
\end{array}\right), r_{2,1}^{k}=\frac{1}{2}\left(\begin{array}{cc}
-\mathrm{i} & -1 \\
-1 & \mathrm{i}
\end{array}\right), r_{2,2}^{k}=\frac{1}{2}\left(\begin{array}{cc}
1 & \mathrm{i} \\
-\mathrm{i} & 1
\end{array}\right),
$$

and the projections

$$
p_{1,1}^{k}=\frac{1}{2}\left(e_{1,1}^{k}+e_{1,2}^{k}+e_{2,1}^{k}+e_{2,2}^{k}\right) \quad \text { and } \quad p_{2,2}^{k}=\frac{1}{2}\left(e_{1,1}^{k}-e_{1,2}^{k}-e_{2,1}^{k}+e_{2,2}^{k}\right),
$$


as well as, following L. Vainermann, their odd and even sums:

$$
p_{i}=\sum_{k=1, k \text { odd }}^{n-1} p_{i, i}^{k}, \quad i=1,2 \quad \text { and } \quad q_{i}=\sum_{k=1, k \text { even }}^{n-1} p_{i, i}^{k}, \quad i=1,2 .
$$

We finally consider the projections $q(\alpha, \beta, k)$ of the $k$-th factor $M_{2}(\mathbb{C})$ given by

$$
\frac{1}{2}\left(\begin{array}{cc}
1-\sin \alpha & \mathrm{e}^{-\mathrm{i} \beta} \cos \alpha \\
\mathrm{e}^{\mathrm{i} \beta} \cos \alpha & 1+\sin \alpha
\end{array}\right),
$$

for $\alpha$ and $\beta$ in $\mathbb{R}$. Then,

$$
q(\alpha, \beta, k)+q(-\alpha, \beta+\pi, k)=e_{1,1}^{k}+e_{2,2}^{k} .
$$

For the new coalgebra structure, the coproduct is obtained by twisting the symmetric coproduct $\Delta_{s}$ of $\mathbb{C}\left[D_{2 n}\right]$ :

$$
\Delta(x)=\Omega \Delta_{s}(x) \Omega^{*}
$$

where $\Omega$ is the unitary of $\mathbb{C}[H] \otimes \mathbb{C}[H]$ given in A.3, We set $J_{0}=\mathbb{C}[H]$.

The coinvolution and the counit are left unchanged 8 . The left regular representation $\lambda$ of $\mathbb{C}\left[D_{2 n}\right]$ and the 2-cocycle $\Omega$ are given explicitly in Appendix A.1 and A.3, respectively. The coproduct expansions relevant to the description of the coideal subalgebras are computed in Appendix A.5.2 for general formulas and by computer for examples (see Appendix B.4.2).

5.3. The Kac algebra $K D(2)$ of dimension 8. We now describe completely the Kac algebra $K D(2)$. As in Appendix B.4, we check on computer that the coproduct of $K D(2)$ is symmetric and that its intrinsic group is $\langle c, \lambda(b)\rangle$, where $c=e_{1}-e_{2}+e_{3}-e_{4}-i e_{1,1}+i e_{2,2}$ is of order 4 with $c^{2}=\lambda\left(a^{2}\right)$.

Therefore, $K D(2)$ is isomorphic to $\mathbb{C}\left[D_{4}\right]$, and the lattice of the coideal subalgebras of $K D(2)$, depicted in Figure 7, coincides with that of the subgroups of $D_{4}$ (see 2.8.4).

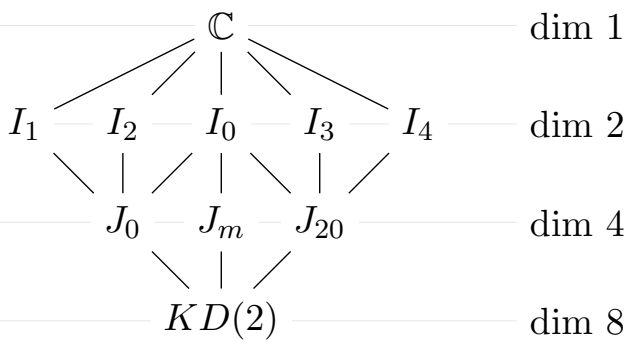

Figure 7 . The lattice of coideal subalgebras of $K D(2)$

The coideal subalgebras of dimension 2 are:

- $I_{0}=\mathbb{C}\left(e_{1}+e_{2}+e_{3}+e_{4}\right) \oplus \mathbb{C}\left(e_{1,1}+e_{2,2}\right)$ generated by $\lambda\left(a^{2}\right)$;

- $I_{1}=\mathbb{C}\left(e_{1}+e_{4}+p_{2}\right) \oplus \mathbb{C}\left(e_{2}+e_{3}+p_{1}\right)$ generated by $\lambda\left(b a^{2}\right)$;

- $I_{2}=\mathbb{C}\left(e_{1}+e_{4}+p_{1}\right) \oplus \mathbb{C}\left(e_{2}+e_{3}+p_{2}\right)$ generated by $\lambda(b)$;

- $I_{3}=I\left(e_{1}+e_{2}+r_{2,2}^{1}\right)$ generated by $\lambda(b) c$;

- $I_{4}=I\left(e_{1}+e_{2}+r_{1,1}^{1}\right)$ generated by $\lambda(b) c^{3}$.

The coideal subalgebras of dimension 4 are:

- $J_{0}=\mathbb{C}\left(e_{1}+e_{4}\right) \oplus \mathbb{C}\left(e_{2}+e_{3}\right) \oplus \mathbb{C} p_{1} \oplus \mathbb{C} p_{2}$ generated by $\lambda\left(a^{2}\right)$ and $\lambda(b)$;

- $J_{20}=\mathbb{C}\left(e_{1}+e_{2}\right) \oplus \mathbb{C}\left(e_{3}+e_{4}\right) \oplus \mathbb{C}\left(r_{1,1}^{1}\right) \oplus \mathbb{C}\left(r_{2,2}^{1}\right)$ generated by $\lambda\left(a^{2}\right)$ and $\lambda(b) c$;

- $J_{m}=\mathbb{C}\left(e_{1}+e_{3}\right) \oplus \mathbb{C}\left(e_{2}+e_{4}\right) \oplus \mathbb{C}\left(e_{1,1}^{1}\right) \oplus \mathbb{C}\left(e_{2,2}^{1}\right)$ generated by $c$.

\footnotetext{
${ }^{8}$ In most proofs, checking the properties of the coinvolution and the counit is straightforward and we omit them.
} 
The rationale behind the notations will become clearer later on.

\subsection{Coidalgebras induced by subgroups.}

\subsubsection{Coidalgebras containing $H$.}

Theorem 5.4.1. Let $K$ be a finite dimensional Kac algebra obtained by twisting a group algebra $\mathbb{C}[G]$ with a 2-(pseudo)-cocycle $\Omega$ of a commutative subgroup $H$. Then, the coideal subalgebras of $K$ containing $H$ are in correspondence with the subgroups of $G$ containing $H$. Namely, the coideal subalgebra $I_{G^{\prime}}$ corresponding to the subgroup $G^{\prime}$ is obtained by twisting the group algebra $\mathbb{C}\left[G^{\prime}\right] \subset \mathbb{C}[G]$ with $\Omega$; in particular, its Jones projection is still $p_{G}=\frac{1}{\left|G^{\prime}\right|} \sum_{g^{\prime} \in G^{\prime}} \lambda\left(g^{\prime}\right)$.

Proof. Recall that the twisted coproduct of $K$ is defined by:

$$
\Delta(x)=\Omega \Delta_{s}(x) \Omega^{*},
$$

where $\Delta_{s}(x)$ is the standard coproduct of the group algebra $\mathbb{C}[G]$; recall also that $\Omega$ is a unitary of $H \otimes H$. Let $G^{\prime}$ be a subgroup of $G$ containing $H$, and $I=\mathbb{C}\left[G^{\prime}\right]$. Then, $\Delta(I) \subset \Omega(I \otimes I) \Omega^{*}=I \otimes I$. Therefore $I$ is the Kac subalgebra of $K$, obtained by twisting $\mathbb{C}\left[G^{\prime}\right]$ with the cocycle $\Omega$. In particular, its trace, counit, and Jones projections are the same as for $\mathbb{C}\left[G^{\prime}\right]$ (see 5.1, and 2.8.4 for the formula).

Reciprocally, let $I$ be a coideal subalgebra of $K$ containing $H$. We may untwist the coproduct on $I$

$$
\Delta_{s}(x)=\Omega^{*} \Delta(x) \Omega .
$$

Then, $\Delta_{s}(I) \subset K \otimes I$, making $I$ into a coideal subalgebra of $\mathbb{C}[G]$. Therefore, $I$ is the algebra of some subgroup $G^{\prime}$ of $G$.

Corollary 5.4.1. Let $K$ be a finite dimensional Kac algebra obtained by twisting a group algebra $\mathbb{C}[G]$ with a 2-(pseudo)-cocycle $\Omega$ of a commutative subgroup $H$. The intrinsic group $G(K)$ of $K$ contains $H$. Therefore, its group algebra coincides with the algebra of the subgroup $G^{\prime}$ of those elements of $G$ such that $\Delta(g)$ is symmetric.

Remark 5.4.1. This gives a rather efficient way to compute the order of the intrinsic group of $K$ (complexity: $|G|$ coproduct computations, that is $O\left(|G||H|^{4}\right)$ ). Beware however that $G^{\prime}$ need not coincide nor even be isomorphic to $G(K)$; consider for example the Kac algebras $K D(2)$ and $K Q(2)$ which are isomorphic (see 8.2). Therefore, to compute the actual intrinsic group, and not only its group algebra, one still needs to compute the minimal idempotents of the (commutative) dual algebra of $\left(\mathbb{C}\left[G^{\prime}\right], \Delta\right)$.

5.4.2. Embedding of $K D(d)$ into $K D(n)$. We now apply the previous results to $K D(n)$.

Corollary 5.4.2. Let $n$ be a positive integer and $d$ a divisor of $n$. Then $K D(d)$ embeds as a Kac subalgebra into $K D(n)$ via the morphism defined by $\varphi_{k}(\alpha)=a^{k}$ and $\varphi_{k}(\beta)=b$, where $k=\frac{n}{d}$, and $\alpha$ and $\beta$ denote the generators of $K D(d)$.

Furthermore, all the coideal subalgebras of $K D(n)$ containing $H$ are obtained this way.

Proof. The subgroups of $D_{2 n}$ containing $H$ are the $G_{k}=\left\langle a^{k}, b\right\rangle$ for $k$ as above. Furthermore, by Theorem 5.4.1, $\left(\mathbb{C}\left[G_{k}\right], \Delta\right)$ is a Kac subalgebra of $K D(n)$; it is obviously isomorphic to $K D(d)$ (the unitary $\Omega$ is constructed from the same elements $a^{n}=\left(a^{k}\right)^{d}$ and $b$ and the same cocycle).

Note that $K D(d)$ could alternatively be embedded into $K D(n)$ by choosing any $k$ of the form $\frac{n}{d}\left(1+2 k^{\prime}\right)$; this gives the same subalgebra of $K D(n)$ and amounts to compose the embedding with some automorphism of $K D(n)$ (see 5.4.3). In fact, under the assumption that $n \geq 3$, the automorphism group of $K D(n)$ stabilizes the embedding of $K D(d)$. 
Example 5.4.2. When $n=2 m, K D(n)$ contains the Kac subalgebras $K_{0}=\mathbb{C}\left[a^{m}, b\right]$ isomorphic to $K D(2)$, and $K_{4}=\mathbb{C}\left[a^{2}, b\right]$ isomorphic to $K D(m)$. Those subalgebras are studied in 7.

5.4.3. Intrinsic group of $K D(n)$ and its dual. The intrinsic group of the dual Kac algebra $\widehat{K D(n)}$ of $K D(n)$ is $\mathbb{Z}_{2} \times \mathbb{Z}_{2}$ Vai98, p.718]. We give here the intrinsic group of $K D(n)$ itself.

Proposition 5.4.3. The intrinsic group of $K D(n)$ is $\mathbb{Z}_{2} \times \mathbb{Z}_{2}$ if $n$ is odd and $D_{4}$ if $n$ is even.

Proof. Applying Corollaries 5.4.1 and 5.4.2, the algebra of the intrinsic group of $K D(n)$ is isomorphic to $K D(k)$ for some $k$ dividing $n$. Furthermore $K D(k)$ is cocommutative if and only if $k=1$ or $k=2$. Finally, $K D(1)$ and $K D(2)$ are respectively isomorphic to $\mathbb{C}\left[D_{2}\right]$ and $\mathbb{C}\left[D_{4}\right]$.

5.5. Automorphism group. In this subsection, we describe the automorphism group of $K D(n)$. The point is that each automorphism of $K D(n)$ induce a symmetry in the lattice $1(K D(n))$ of coideal subalgebras of $K D(n)$. Namely, it maps a coideal subalgebra on a coideal subalgebra (mapping the Jones projection of the first to the Jones projection of the second).

5.5.1. Automorphisms induced by automorphisms of $D_{2 n}$ fixing $\mathbb{C}[H]$.

Proposition 5.5.1. Let $k$ be coprime with $2 n$, and $\Theta_{k}$ be the algebra morphism defined by:

$$
\Theta_{k}(\lambda(a))=\lambda\left(a^{k}\right) \quad \text { and } \quad \Theta_{k}(\lambda(b))=\lambda(b) .
$$

Then, $\Theta_{k}$ is a Kac algebra automorphism of $K D(n)$ which fixes $H$.

Proof. Such a $\Theta_{k}$ is an automorphism of the group $D_{2 n}$, and therefore an automorphism of its Kac algebra $\mathbb{C}\left[D_{2 n}\right]$. Since $H$ is fixed, so is $\Omega$, so that the coproduct $\Delta$ of $K D(n)$ is preserved as well.

Example 5.5.1. Take $\Theta=\Theta_{-1}$. It is an involutive automorphism of $K D(n)$; it can be alternatively described as the conjugation by $\lambda(b)$.

5.5.2. An automorphism which does not fix $\mathbb{C}[H]$. We now construct an automorphism $\Theta^{\prime}$ which acts non trivially on $\mathbb{C}[H]$.

Proposition 5.5.2. The following formulas define an involutive Kac algebra automorphism of $K D(n)$ :

$$
\Theta^{\prime}(\lambda(a))=\lambda(a)-\frac{1}{2}\left(\lambda(a)-\lambda\left(a^{-1}\right)\right)\left(1+\lambda\left(a^{n}\right)\right) \quad \text { and } \quad \Theta^{\prime}(\lambda(b))=\lambda\left(a^{n} b\right) .
$$

5.5.3. Tips and tricks to prove an isomorphism. The following (easy) proposition gives a conveniently small list of properties to be checked to be sure that an homomorphism defined by the images of some generators extends to a proper Kac-algebra isomorphism.

Proposition 5.5.3. Let $K$ and $K^{\prime}$ be two Kac algebras of identical finite dimension, and $A$ and $A^{\prime}$ be sets of algebra generators of $K$ and $K^{\prime}$ respectively. Fix $\Phi(a) \in K^{\prime}$ for $a \in A$. Then, $\Phi$ extends to a Kac-algebra isomorphism from $K$ to $K^{\prime}$ if and only if:

(i) The elements $\{\Phi(a) \mid a \in A\}$ satisfy the relations of $A$, and therefore define a (non necessarily unital) algebra morphism $\Phi$ from $K$ to $K^{\prime}$;

(ii) $\Phi$ preserves the involution on $A: \Phi\left(a^{*}\right)=\Phi(a)^{*}$, for all $a \in A$;

(iii) $\Phi$ preserves the coproduct on $A:(\Phi \otimes \Phi)(\Delta(a))=\Delta(\Phi(a))$, for all $a \in A$;

(iv) All elements $a^{\prime} \in A^{\prime}$ have a preimage by $\Phi$ in $K$. 
Proof. Using (i), $\Phi$ extends to a (possibly non unital) algebra morphism from $K$ to $K^{\prime}$. By (iv), $\Phi$ is bijective, and by the uniqueness of the unit it is an unital algebra isomorphism. Similarly, by (iii) $\Phi$ is a coalgebra isomorphism. The counit and the antipode are preserved, as they are unique in a Hopf algebra (see [Kas95, III.3.2]).

By semi-simplicity of the algebra, the trace is preserved; indeed, the central idempotents are unique (up to permutation among those of same rank), and the normalization of the trace on each corresponding matrix block is determined by the rank. The coinvolution coincides with the antipode 2.8.1 and is preserved. Finally, the involution being an antialgebra morphism, (ii) ensures it is preserved as well.

5.5.4. Automatic checks on computer. Checking the properties listed in Proposition 5.5.3 often boils down to straightforward, pointless, and tedious calculations. We therefore wish to delegate them to the computer whenever possible. To obtain Proposition 5.5.2, we shall in principle prove those properties for all values of $n$; however it turns out that, in most cases, it is sufficient to check them only for small values of $n$, which is easier to automatize. We introduce here just a bit of formalism that justifies this approach. For the sake of simplicity, we do that for the special case of $K D(n)$, but we later reuse straightforward analogues in other isomorphism proofs.

The idea is to consider $n$ as a formal parameter, which can be though of as letting $n$ go to infinity. Namely, let $D_{\infty}$ be the (infinite) group generated by $a, b, a_{\infty}$, subject to the relations $b^{2}=a_{\infty}{ }^{2}=1, a_{\infty} b=b a_{\infty}$, and $a b=b a^{-1}$. We denote by $\Pi_{D, n}$ the canonical quotient map from $D_{\infty}$ to $D_{2 n}$ which sends $a_{\infty}$ to $a^{n}$ (its kernel is the subgroup generated by $\left.a_{\infty} a^{-n}\right)$. Let $\mathbb{C}\left[D_{\infty}\right]$ be the group algebra of $D_{\infty}$. The quotient map $\Pi_{D, n}$ extends to a surjective algebra morphism from $\mathbb{C}\left[D_{\infty}\right]$ to $\mathbb{C}\left[D_{2 n}\right]$. It is further injective whenever restricted to the subspace of $\mathbb{C}\left[D_{\infty}\right]$ spanned by $a^{i} a_{\infty}{ }^{j} b^{k}$ with $2|i|<n$. Define the degree of an algebraic expression $A$ involving $a, b$ and $a_{\infty}$ as the maximal $d$ such that $a^{d}$ or $a^{-d}$ occurs in it.

Proposition 5.5.4. An algebraic expression A of degree d which vanishes in some $K D(N)$, $N>2 d$, vanishes in $K D(n)$ for any $n \in \mathbb{N}$.

Proof. Indeed, it can be lifted up via $\Pi_{D, N}$ to $\mathbb{C}\left[D_{\infty}\right]$ and then projected down to $\mathbb{C}\left[D_{n}\right]$ for any other $n \in \mathbb{N}$ via $\Pi_{D, n}$.

Note that the bound $N>2 d$ is tight because of the cancellation $a^{d}-a_{\infty} a^{-d}=0$ if $n=2 d$.

As illustrated in the following example, we often use implicitly straightforward variants of this proposition.

Example 5.5.4. The coproduct $\Delta(a)=\Omega(a \otimes a) \Omega^{*}$ is of degree 1. One can read off the general formula for its expansion from the computation for $N=3$; this expansion has 64 terms, and is non symmetric. However, for $N=2$ there are cancellations: the expansion degenerates to 16 terms and becomes symmetric.

\subsubsection{Technical lemma.}

Lemma 5.5.5. For all $k \in \mathbb{Z}$,

$$
\Theta^{\prime}(\lambda(a))^{k}=\lambda\left(a^{k}\right)-\frac{1}{2}\left(\lambda\left(a^{k}\right)-\lambda\left(a^{-k}\right)\right)\left(1+\lambda\left(a^{n}\right)\right),
$$

and $\Theta^{\prime}$ preserves the involution.

Proof. Write $f_{k}=\lambda\left(a^{k}\right)-\frac{1}{2}\left(\lambda\left(a^{k}\right)-\lambda\left(a^{-k}\right)\right)\left(1+\lambda\left(a^{n}\right)\right)$. Since everything commutes and $\lambda\left(a^{n}\right)$ is its own inverse, one checks easily that $f_{k} f_{1}=f_{k+1}$. Specially we have $f_{-1}=f_{1}^{*}=f_{1}^{-1}$, so $\Theta^{\prime}\left(\lambda(a)^{*}\right)=\Theta^{\prime}(\lambda(a))^{*}$. The lemma follows by induction. 


\subsubsection{Proof of Proposition 5.5.2.}

Proof. We check that $\Theta^{\prime}$ satisfies the properties listed in Proposition 5.5.3.

(i) Thanks to Lemma [5.5.5, $\Theta^{\prime}(\lambda(a))^{2 n}=1$. The relation $\Theta^{\prime}(\lambda(b))^{2}=1$ is obvious. The remaining relation $\left.\Theta^{\prime}(\lambda(b)) \Theta^{\prime}(\lambda(a))=\Theta^{\prime}(\lambda(a))^{-1}\right) \Theta^{\prime}(\lambda(b))$ is of degree 1. Using Proposition 5.5.4, it is sufficient to check on computer that it holds in $K D(3)$.

(ii) Follows from Lemma 5.5.5

(iii) Using a direct extension of Proposition $\left[5.5 .4\right.$ for $\mathbb{C}\left[D_{2 n}\right] \otimes \mathbb{C}\left[D_{2 n}\right]$, we check on computer that, in $K D(3)$ and for $x=a, b,\left(\Theta^{\prime} \otimes \Theta^{\prime}\right)(\Delta(\lambda(x)))=\Delta\left(\Theta^{\prime}(x)\right)$.

(iv) We prove that $\Theta^{\prime}$ is an involution (and therefore an isomorphism) by checking on computer that the equation $\Theta^{\prime}\left(\Theta^{\prime}(\lambda(a))\right)=\lambda(a)$ holds in $K D(3)$. The equation $\Theta^{\prime}\left(\Theta^{\prime}(\lambda(b))\right)=\lambda(b)$ is obvious.

5.5.7. The automorphism group of $K D(n)$.

Theorem 5.5.7. For $n \geq 3$, the automorphism group $\operatorname{Aut}(K D(n))$ of $K D(n)$ is given by:

$$
A_{2 n}=\left\{\Theta_{k}, \Theta_{k} \Theta^{\prime} \mid k \wedge 2 n=1\right\} .
$$

In particular, it is of order $2 \varphi(2 n)$ (where $\varphi$ is the Euler function), and isomorphic to $\mathbb{Z}_{2 n}^{*} \rtimes \mathbb{Z}_{2}$, where $\mathbb{Z}_{2 n}^{*}$ is the multiplicative group of units of $\mathbb{Z}_{2 n}$.

Proof. From Propositions 5.5.1 and 5.5.2, Aut $(K D(n))$ contains $A_{2 n}$. Let us prove the converse.

Let $\Phi$ be an automorphism of $K D(n)$. It induces an automorphism $\sigma$ of the intrinsic group $G(K D(n))$ (respectively $H$ for $n$ odd, and $D_{4}$ for $n$ even). Note that $(\Phi \otimes \Phi)(\Omega)$ is completely determined by $\sigma$. Furthermore, we can use it to "untwist" the coproduct of $K D(n)$ into a cocommutative coproduct by defining for all $y$ in $K D(n)$ :

$$
\Delta_{\sigma}(y)=(\Phi \otimes \Phi)(\Omega)^{*} \Delta(y)(\Phi \otimes \Phi)(\Omega) .
$$

This coproduct is indeed cocommutative because

$$
\Delta_{\sigma}(y)=(\Phi \otimes \Phi)(\Omega)^{*} \Delta(\Phi(x))(\Phi \otimes \Phi)(\Omega)=(\Phi \otimes \Phi)\left(\Omega^{*} \Delta(x) \Omega\right)=(\Phi \otimes \Phi)\left(\Delta_{s}(x)\right)
$$

where $x=\Phi^{-1}(y)$, and $\Delta_{s}$ is the usual cocommutative coproduct of $\mathbb{C}\left[D_{2 n}\right]$.

We claim that, for $n \geq 3$, this later property rules out all the automorphism of $G(K D(n))$ but $\sigma=$ id and $\sigma$ defined by $\sigma\left(a^{n}\right)=a^{n}$ and $\sigma(b)=a^{n} b$. Consider indeed the case $n$ odd (resp. even), and take $\tau$ one of the 4 (resp. 6) remaining automorphisms of $G(K D(n))=H$ $\left(\right.$ resp $\left.=D_{4}\right)$. The expression $\Delta_{\tau}(\lambda(a))$ is of degree 1 , so it is sufficient to check on computer that it is non symmetric for $n=3$ (resp $n=4)$.

If $\sigma=$ id, then $\Delta_{\sigma}=\Delta_{s}$, so $\Phi$ is an automorphism of $\mathbb{C}\left[D_{2 n}\right]$ fixing $H$. Therefore $\Phi=\Theta_{k}$ for some $k$ coprime to $2 n$. Otherwise, $\Phi \Theta^{\prime}$ fixes $H$, so $\Phi$ is of the form $\Theta_{k} \Theta^{\prime}$.

5.5.8. Computing isomorphisms. The reasoning developed in the previous proof turns into an algorithm to compute Kac algebra isomorphisms. All the isomorphisms in this article were conjectured from the application of this algorithm on small examples (see B.5.1, B.5.2, and B.5.4).

Algorithm 5.5.8. Let $K=K(\mathbb{C}[G], H, \Omega)$ be a finite dimensional Kac algebra obtained by twisting a group algebra $\mathbb{C}[G]$ with a 2 -(pseudo)-cocycle $\Omega$ of a commutative subgroup $H$, and let $K^{\prime}$ be any finite dimensional Kac algebra. The following algorithm returns all the Kac algebra isomorphisms $\phi$ from $K$ to $K^{\prime}$. In particular, the result is non empty if and only if the two algebras are isomorphic.

- Compute the intrinsic group $H^{\prime}$ of $K^{\prime}$;

- For each embedding $\rho$ of $H$ into $H^{\prime}$ : 
- Construct $K^{\prime \prime}=K\left(K^{\prime}, \rho(H), \Omega^{*}\right)$ whose coproduct $\Delta^{\prime \prime}$ is obtained by (un)twisting the coproduct $\Delta^{\prime}$ of $K^{\prime}$ by the inverse cocycle $\Omega^{*}$;

- If the untwisted coproduct $\Delta^{\prime \prime}$ is cocommutative:

* Compute the intrinsic group $G^{\prime \prime}$ of $K^{\prime \prime}$;

* Compute the group isomorphisms from $G$ to $G^{\prime \prime}$ which are compatible with $\rho$.

Proof. If there exist a Kac algebra isomorphism $\phi$ from $K$ to $K^{\prime}$, then the Kac algebra $K^{\prime \prime}=K\left(K^{\prime}, \phi(H), \Omega^{*}\right)$ is isomorphic to $\mathbb{C}[G]$.

This algorithm is not blazingly fast, but we are not aware of any other algorithms to test systematically the isomorphism of two Kac algebras (although their definitely should exist one). It also has some nice theoretical consequences: first there are finitely many isomorphisms, and second the existence of an isomorphism does not depend on the actual ground field (typically $\mathbb{C}$ or $\mathbb{Q}(i, \epsilon)$ ).

5.6. The three coideal subalgebras $K_{2}, K_{3}, K_{4}$ of dimension $2 n$. We come back to the study of the lattice $1(K D(n))$. The action of $G(\widehat{K D(n)})$ on $K D(n)$ produces three distinct exterior actions of $\mathbb{Z}_{2}$ on $N_{3}$, and the corresponding three fixed point algebras are three subfactors of index 2 in $N_{3}$.

Therefore, $K D(n)$ admits three coideal subalgebras of dimension $2 n$, with Jones projections $e_{1}+e_{2}, e_{1}+e_{3}$ et $e_{1}+e_{4}$ respectively (those are the only possible projections of trace $1 / 2 n)$. We denote by $K_{i}$ the coideal subalgebra $I\left(e_{1}+e_{i}\right)$ for $i=1,2,3$. We postpone the study of $K_{3}$ and $K_{4}$ whose structure depend on the parity of $n$.

5.7. The Kac subalgebra $K_{2}=I\left(e_{1}+e_{2}\right)$ of dimension $2 n$. In this section, we describe completely the coideal subalgebra $K_{2}$ and its coideal subalgebras. The following result was suggested by computer exploration (see Appendix B.4.3).

Proposition 5.7. The coideal subalgebra $K_{2}=I\left(e_{1}+e_{2}\right)$ is isomorphic to the Kac algebra of functions on the group $D_{n}$. Its lattice of coideal subalgebras is the dual of the lattice of subgroups of $D_{n}$.

Proof. First, $K_{2}=I\left(e_{1}+e_{2}\right)$ is indeed a Kac subalgebra of $K D(n)$ because $\Delta\left(e_{1}+e_{2}\right)$ is symmetric (see Appendix A.5.2). Looking further at the expression of this coproduct yields the following block matrix structure for $K_{2}$ :

$$
K_{2}=\mathbb{C}\left(e_{1}+e_{2}\right) \oplus \mathbb{C}\left(e_{3}+e_{4}\right) \oplus \bigoplus_{j=1, j \text { odd }}^{n-1}\left(\mathbb{C} r_{1,1}^{j} \oplus \mathbb{C} r_{2,2}^{j}\right) \oplus \bigoplus_{j=1, j \text { even }}^{n-1}\left(\mathbb{C} e_{1,1}^{j} \oplus \mathbb{C} e_{2,2}^{j}\right) \text {. }
$$

All the blocks are trivial, so $K_{2}$ is commutative. Therefore it is the Kac algebra $L^{\infty}(G)$ of functions on some group $G$. The minimal projections are the characteristic functions $\chi_{g}$ of the elements of $g \in G$. To start with, $\chi_{1}=e_{1}+e_{2}$, since the later is the support of the counit of $I\left(e_{1}+e_{2}\right)$. We denote the others as follows: $e_{3}+e_{4}=\chi_{\beta_{0}}$,

$$
\begin{array}{rlrl}
r_{1,1}^{2 j-1} & =\chi_{\beta_{j}}, & r_{2,2}^{2 j-1}=\chi_{\beta_{j}^{\prime}}, & \text { for } j=1, \ldots, m, \\
e_{1,1}^{2 j}=\chi_{\alpha_{j}}, & e_{2,2}^{2 j}=\chi_{\alpha_{-j}}, & \text { for } j=1, \ldots, m^{\prime} .
\end{array}
$$

with $m^{\prime}=\lfloor(n-1) / 2\rfloor$. For short, we also write $\alpha=\alpha_{1}$.

The group law on $G$ is determined by the coproducts of those central idempotents: $\Delta\left(\chi_{g}\right)=\sum_{h k=g} \chi_{h} \otimes \chi_{k}$ (the expressions of those coproducts are computed in Appendix A.5.2). In particular, $\Delta\left(e_{1}+e_{2}\right)$ gives the inverses: the $\beta_{j}$ are idempotents, while $\alpha_{j}$ is the inverse of $\alpha_{-j}$. To obtain the remaining products, we need to distinguish between $n$ even and odd. 
Case $n=2 m$ : From the expression of $\Delta\left(e_{1,1}^{2}\right)$, one get:

- for $j>2$ even, $\Delta\left(e_{1,1}^{2}\right)\left(e_{2,2}^{j-2} \otimes e_{1,1}^{j}\right)=e_{2,2}^{j-2} \otimes e_{1,1}^{j}$. Therefore, for $k=2, \ldots, m-1$, $\alpha_{k}=\alpha_{k-1} \alpha_{1}$, and by induction $\alpha_{k}=\alpha^{k}$ and $\alpha_{-k}=\alpha^{-k}$.

- $\Delta\left(e_{1,1}^{2}\right)\left(\left(e_{3}+e_{4}\right) \otimes e_{2,2}^{n-2}\right)=\left(e_{3}+e_{4}\right) \otimes e_{2,2}^{n-2}$. Therefore, $\beta_{0}=\alpha \alpha_{m-1}=\alpha^{m}$ and $\alpha$ is of order $n$.

- For $j>2$ odd, $\Delta\left(e_{1,1}^{2}\right)\left(r_{1,1}^{j-2} \otimes r_{1,1}^{j}\right)=r_{1,1}^{j-2} \otimes r_{1,1}^{j}$ and $\Delta\left(e_{1,1}^{2}\right)\left(r_{2,2}^{n-j+2} \otimes r_{2,2}^{n-j}\right)=$ $r_{2,2}^{n-j+2} \otimes r_{2,2}^{n-j}$. It follows that for $k=1, \ldots, m-1, \beta_{k+1}=\beta_{k} \alpha$ and $\beta_{k+1}^{\prime}=\alpha \beta_{k}^{\prime}$ and by induction $\beta_{k+1}=\beta_{1} \alpha^{k}$ et $\beta_{k+1}^{\prime}=\alpha^{k} \beta_{1}^{\prime}$.

- $\Delta\left(e_{1,1}^{2}\right)\left(r_{2,2}^{1} \otimes r_{1,1}^{1}\right)=r_{2,2}^{1} \otimes r_{1,1}^{1}$. Therefore, $\beta_{1}^{\prime}=\alpha \beta_{1}$.

The group $G$ is generated by $\alpha$ of order $n$ and $\beta=\beta_{1}$ of order 2. From the expression $\Delta\left(e_{3}+e_{4}\right)$ given in $\mathrm{A} .5 .2$, one get: $\Delta\left(e_{3}+e_{4}\right)\left(r_{1,1}^{2 m-1} \otimes r_{2,2}^{1}\right)=\left(r_{1,1}^{2 m-1} \otimes r_{2,2}^{1}\right)$, that is $\beta_{1}^{\prime}=\beta_{m} \beta$, which gives the relation $\alpha \beta=\beta \alpha^{-1}$. Therefore, $G$ is the dihedral group $D_{n}$.

Case $n=2 m+1$ : From the expression of $\Delta\left(e_{1,1}^{2}\right)$ one get:

- for $j>2$ even, $\Delta\left(e_{1,1}^{2}\right)\left(e_{2,2}^{j-2} \otimes e_{1,1}^{j}\right)=e_{2,2}^{j-2} \otimes e_{1,1}^{j}$. Therefore, for $k=2, \ldots, m$ $\alpha_{k}=\alpha_{k-1} \alpha_{1}$, and by induction $\alpha_{k}=\alpha^{k}$ and $\alpha_{-k}=\alpha^{-k}$.

- $\Delta\left(e_{1,1}^{2}\right)\left(e_{2,2}^{n-1} \otimes e_{2,2}^{n-1}\right)=e_{2,2}^{n-1} \otimes e_{2,2}^{n-1}$. Therefore, $\alpha$ is of order $n$.

The expression of the coproduct of $e_{3}+e_{4}$ gives the following relations for $j=1, \ldots, m$, $\beta_{m+1-j}=\beta_{0} \alpha_{-j}$ and $\beta_{m+1-j}^{\prime}=\beta_{0} \alpha_{j}$.

Since $\beta_{m+1-j}$ is of order 2 , for all $k=0, \ldots, n-1$, the following commutation relation holds: $\beta_{0} \alpha^{-k}=\alpha^{k} \beta_{0}$. Therefore, $G$ is generated by $\alpha$ of order $n$ and $\beta=\beta_{0}$ of order 2 with the relation $\alpha \beta=\beta \alpha^{-1}$ : it is again the dihedral group $D_{n}$.

5.8. The coideal subalgebras of $K_{2}$. Each coideal subalgebra of $K_{2}$ is the subspace of invariant functions on the right cosets of some subgroup of $D_{n}$. In Appendix B.4.2 we show how to get all the Jones projection on computer for $n$ small. We do it here explicitly for some examples for all $n$ :

- Take $m$ such that $n=2 m$ or $n=2 m+1$. The $2 m+1$ subgroups of order 2 of $D_{n}$ induce $2 m+1$ coideal subalgebras of dimension $n$. Their Jones projection are respectively: $e_{1}+e_{2}+e_{3}+e_{4}, e_{1}+e_{2}+r_{1,1}^{2 j+1}$ and $e_{1}+e_{2}+r_{2,2}^{2 j+1}$ with $j=0, \ldots, m-1$.

- The subgroup generated by $\alpha$ induces the coideal subalgebra $I_{0}=I\left(e_{1}+e_{2}+q_{1}+q_{2}\right)$ of dimension 2.

- If $n$ is even, the subgroup generated by $\alpha^{2}$ induces a coideal subalgebra $J_{20}$ of dimension 4 contained in $K_{0}$ (see 7.1.2).

5.9. The coideal subalgebra $K_{1}=I\left(e_{1}+e_{2}+e_{3}+e_{4}\right)$ of dimension $n$. The intersection $K_{1}$ of the three coideal subalgebras $K_{2}, K_{3}$ et $K_{4}$ is the image by $\delta$ of the algebra of the intrinsic group of $\widehat{K D(n)}$. Its dimension is $n$; its Jones projection is $e_{1}+e_{2}+e_{3}+e_{4}$ since it is of trace $1 / n$ and dominates $e_{1}+e_{2}, e_{1}+e_{3}$, and $e_{1}+e_{4}$. The connected component of $e_{1}$ in the Bratelli diagram of $I\left(e_{1}+e_{2}+e_{3}+e_{4}\right) \subset K D(n)$ is the principal graph of the inclusion $N \subset N \rtimes \mathbb{Z}_{2} \times \mathbb{Z}_{2}$. The structure of $K_{1}$ depends on the parity of $n$ and is further elucidated in 7.4 and 6.4 .

5.10. Coidalgebras of dimension dividing $2 n$. The following proposition allows for recovering many, if not all, of the coideal subalgebras recursively from those of $K_{2}, K_{3}$, and $K_{4}$.

Proposition 5.10. If the dimension of a coideal subalgebra $I$ of $K D(n)$ divides $2 n$ (and not just $4 n$ ), then either its Jones projection $p_{I}$ dominates $e_{1}+e_{2}+e_{3}+e_{4}$ and then $I$ is contained in $K_{1}$, or there exists a unique $i \in\{2,3,4\}$ such that $p_{I}$ dominates $e_{1}+e_{i}$ 
and $I$ is a subcoideal subalgebra of $K_{i}$. In particular, if $n$ is a power of 2 , then any coideal subalgebra is contained in one of the $K_{i}$ 's.

Proof. The Jones projection $p_{I}$ of a coideal subalgebra $I$ dominates $e_{1}$. Given the block matrix structure of $K D(n)$, it is the sum of $x$ projections of trace $1 / 4 n$ (with $x \geq 1$ ) et and $y$ projections of trace $1 / 2 n$. Its trace $\operatorname{tr}\left(p_{I}\right)=(\operatorname{dim} I)^{-1}$, is therefore $(x+2 y) / 4 n$ (see 2.8.2). If the dimension of $I$ divides $2 n$ (which is always the case when $n$ is a power of 2), $x$ must be even, and therefore $p_{I}$ takes one of the two following forms:

- $e_{1}+e_{2}+e_{3}+e_{4}+s$, with $s$ projection and $\operatorname{tr}(s)=(\operatorname{dim} I)^{-1}-n^{-1}$;

- $e_{1}+e_{i}+s^{\prime}$, with $i=2,3$, or 4 , and $s^{\prime}$ projection of trace $(\operatorname{dim} I)^{-1}-(2 n)^{-1}$.

The proposition follows from 2.4.2 (5).

In 6.5. we will establish an even more precise result when $n$ is odd.

5.11. Coidalgebras induced by Jones projections of subgroups. We have seen in 5.4.2 that the algebras of the subgroups containing $H$ are coideal subalgebras of $K D(n)$. The algebras of the other subgroups are usually not coideal subalgebras in $K D(n)$; yet, in many cases, their Jones projections remain Jones projections in $K D(n)$.

Proposition 5.11. The Jones projections of the following subgroups of $D_{2 n}$ are Jones projections of coideal subalgebras of $K D(n)$ of dimension the order of the subgroup. If the subgroup is commutative, then so is the coideal subalgebra.

- $H_{r}=\left\{1, \lambda\left(a^{n}\right), \lambda\left(b a^{r}\right), \lambda\left(b a^{r+n}\right)\right\}$ for $r=0, \ldots, n-1$;

- $A_{k}$, subgroup generated by $a^{k}$, for $k$ such that $2 n=k d$;

- $B_{k}$, subgroup generated by $a^{k}$ and $b$, for $k$ such that $2 n=k d$.

For example, in $K D(9)$, the Jones projection of $\mathbb{C}\left[A_{3}\right]$ is that of $M_{2}$, and that of $\mathbb{C}\left[A_{6}\right]$ generates $L_{0}$ (see Figure 6.10). We refer to 6.6 and 6.7 for further applications, and to 9.3 .1 and 7.7 .6 for examples of Jones projections given by dihedral subgroups.

Not every subgroup, even commutative, gives a Jones projection in the twisted Kac algebra; for example, in $K D(n)$ with $n \geq 3$, there are only three coideal subalgebras of dimension 2 whereas $D_{2 n}$ has $2 n+1$ subgroups of order 2 . Reciprocally, not all Jones projections are given by subgroups; for example, in $K D(3)$ there are three coideal subalgebras of dimension 3 whereas $D_{6}$ has a single subgroup of order 3.

Problem 5.11. Let $K$ be a Kac algebra by twisting a group algebra $\mathbb{C}[G]$. Characterize the subgroups of $G$ whose Jones projections remain Jones projections in $K$.

We now turn to the proof of the proposition, starting with a general lemma.

Lemma 5.11. Let $K$ be a Kac algebra and $p$ be a projection which dominates the Jones projection $f_{2}$ of $K$, and such that $\Delta(p)=\sum_{i=1}^{d} Q_{i} \otimes P_{i}$, where:

- the families $\left(P_{i}\right)_{i=1, \ldots, d}$ and $\left(Q_{i}\right)_{i=1, \ldots, d}$ are linearly independent;

- $d=1 / \operatorname{tr}(p)$;

- $I=\bigoplus_{i=1}^{d} \mathbb{C} P_{i}$ is a unital involutive subalgebra of $K$.

Then, $I$ is a coideal subalgebra of Jones projection $p$. In particular, $Q_{i}=S\left(P_{i}^{*}\right)$.

Proof. This is a consequence of the coassociativity of $\Delta$. Indeed,

$$
\sum_{i=1}^{d} Q_{i} \otimes \Delta\left(P_{i}\right)=(\mathrm{id} \otimes \Delta) \Delta(p)=(\Delta \otimes \mathrm{id}) \Delta(p)=\sum_{i=1}^{d} \Delta\left(Q_{i}\right) \otimes P_{i} ;
$$

applying $\widehat{Q}_{j} \otimes$ id $\otimes$ id on both sides of this equation, where $\left(\widehat{Q}_{i}\right)_{i \in I}$ is a family of linear forms such that $\widehat{Q}_{i}\left(Q_{j}\right)=\delta_{i, j}$, yields that $\Delta\left(P_{j}\right) \in K \otimes J$. Therefore, $\Delta(J) \subset K \otimes J$, and the result follows by Proposition 2.8.2 and Remark 2.8.2. 
Proof of proposition 5.11. We shall see in A.4 that the coproduct of the Jones projection $Q_{r}$ of $\mathbb{C}\left[H_{r}\right]$ is of the form $\Delta\left(Q_{r}\right)=\sum_{i=1}^{4} S\left(P_{i}\right) \otimes P_{i}$, where $P_{1}=Q_{r}$ and $1=P_{1}+P_{2}+$ $P_{3}+P_{4}$ is a decomposition of the identity into orthogonal projections. Then, $J_{r}=\sum_{i} \mathbb{C} P_{i}$ is an involutive commutative subalgebra of dimension 4 , while $\operatorname{tr}\left(Q_{r}\right)=1 / 4$. The result follows by applying Lemma 5.11 ,

If $d$ is even, the Jones projection of $\mathbb{C}\left[A_{k}\right]$ generates a commutative coideal subalgebra, which is the image of $K_{2}$ in $K D(d / 2)$ through the embedding $\varphi_{k}$ in $K D(n)$ (see 5.4 .2 and the expression of $e_{1}+e_{2}$ in A.2 . For example, the Jones projection of $\mathbb{C}\left[A_{1}\right]$ is $e_{1}+e_{2}=p_{K_{2}}$. Otherwise, $k$ is even, and the Jones projection of $\mathbb{C}\left[A_{k}\right]$ generates a commutative coideal subalgebra which is the image of $K_{1}$ in $K D(d)$ through the embedding $\varphi_{k / 2}$ in $K D(n)$. For example, the Jones projection of $\mathbb{C}\left[A_{2}\right]$ is $e_{1}+e_{2}+e_{3}+e_{4}=p_{K_{1}}$.

If $k$ divises $n, \mathbb{C}\left[B_{k}\right]$ is isomorphic to $K D[d / 2]$ (see [5.4.2). If $k=2 k^{\prime}$ does not divide $n$, the Jones projection of $\mathbb{C}\left[B_{k}\right]$ generates a coideal subalgebra which is the image of $K_{4}$ of $K D(d)$ through the embedding $\varphi_{k / 2}$ in $K D(n)$. 


\section{The KaC Algebras $K D(n)$ FOR $n$ ODD}

The study of $1(K D(n))$ for $n$ odd led us to conjecture, and later prove, that $K D(n)$ is self-dual in that case. This property is not only interesting by itself. First, this is a useful tool for constructing new coideal subalgebras by duality (see e.g. Appendix B.5.3), and unraveling the self-dual lattice $\mathrm{l}(K D(n))$; in particular, this is a key ingredient to get the full lattice when $n$ is prime. Last but not least, this allows us to describe completely the principal graph for some inclusions $N_{2} \subset N_{2} \rtimes I$ with $I$ coideal subalgebra of $K D(n)$ (which requires information on $\delta(I)$ ).

After establishing the self-duality, we describe the symmetric coideal subalgebras, as well as those of dimension $2 n$, of odd dimension, and of dimension 4. Putting everything together, we get a partial description of $1(K D(n))$ (Theorem 6.8) which is conjecturally complete (Conjecture 6.8). This is proved for $n$ prime (Corollary 6.8) and checked on computer up to $n=51$. We conclude with some illustrations on $K D(n)$ for $n=3,5,9,15$.

6.1. Self-duality. In Appendix B.5.2, the self-duality of $K D(n)$ for $n$ odd is checked for $n \leq 21$ by computing an explicit isomorphism. From this exploration, we infer the definition of $\psi$, candidate as Kac isomorphism from $K D(n)$ to its dual.

The dual of $\mathbb{C}\left[D_{2 n}\right]$ is the algebra $L^{\infty}\left(D_{2 n}\right)$ of functions on $D_{2 n}$. We denote by $\chi_{g}$ the characteristic function of $g$, so that $\left\{\chi_{g}, g \in G\right\}$ is the dual basis of $\{\lambda(g), g \in G\}$. We denote by $x \mapsto \hat{x}$ the vector-space isomorphism from $\mathbb{C}\left[D_{2 n}\right]$ to its dual $L^{\infty}\left(D_{2 n}\right)$ which extends $\lambda(g) \mapsto \chi_{g}$ by linearity.

The following theorem is the main result of this section:

Theorem 6.1. Let $n$ be odd. Then $K D(n)$ is self-dual, via the Kac algebra isomorphism $\psi$ :

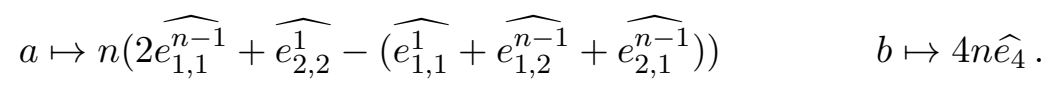

We prove this theorem in the sequel of this section. We start by describing the block matrix decomposition of $\widehat{K D(n)}$. We use it to define a $C^{*}$-algebra isomorphism $\psi$ on the matrix units of $K D(n)$, calculate its expression on $\lambda(a)$ and $\lambda(b)$, and conclude by proving that $\psi$ is a Kac algebra isomorphism. Some heavy calculations are relegated to A.8.

For an alternative non constructive proof of self-duality, one can use the isomorphism with $K A(n)$ (see Theorem 9.2) and the self-duality of the later for $n$ odd which is mentioned in [CDMM04, p. 776].

6.1.1. Block matrix decomposition of $\widehat{K D(n)}$. The product on $L^{\infty}\left(D_{2 n}\right)$ is the point wise (Hadamard) product; the coproduct is defined by duality:

$$
\Delta\left(\chi_{g}\right)=\sum_{s \in D_{2 n}} \chi_{s} \otimes \chi_{s^{-1} g}
$$

For $f \in L^{\infty}\left(D_{2 n}\right)$ and $s \in D_{2 n}$, the involution is given by $f^{*}(s)=\overline{f(s)}$, the coinvolution $S$ by $S(f)(s)=f\left(s^{-1}\right)$, and $\operatorname{tr}\left(\chi_{s}\right)$ by $1 / 2 n$.

The coproduct of $K D(n)$ is that of $\mathbb{C}\left[D_{2 n}\right]$ twisted by $\Omega$; Therefore by duality, the product of $\widehat{K D(n)}$ is twisted from that of $L^{\infty}(G)$. From A.3, it can be written as:

$$
\Delta(\lambda(g))=\sum_{i, j, r, s=1}^{4} c_{i, j} c_{s, r} \lambda\left(h_{i} g h_{r}\right) \otimes \lambda\left(h_{j} g h_{s}\right),
$$


with $h_{1}=1, h_{2}=a^{n}, h_{3}=b, h_{4}=b a^{n}$. By duality, we obtain the twisted product on $L^{\infty}\left(D_{2 n}\right)$ :

$$
\chi_{g_{1}} \odot \chi_{g_{2}}=\sum_{i, j, r, s=1}^{4} c_{i, j} c_{s, r} \chi_{h_{i} g_{1} h_{r}} \chi_{h_{j} g_{2} h_{s}} .
$$

Let us study this new product. If one of the $g_{k}$ is in $H$ and the other is not, then the product $\chi_{g_{1}} \odot \chi_{g_{2}}$ is zero. If both are in $H$, the product is left unchanged since the coproduct is unchanged on $H$. Therefore, $\chi_{1}, \chi_{a^{n}}, \chi_{b}$ and $\chi_{b a^{n}}$ are central projections of $\widehat{K D(n)}$.

Consider now $s$ et $t$ in $D_{2 n}-H$. The product $\chi_{s} \odot \chi_{t}$ is non zero if and only if there exists $h$ and $h^{\prime}$ in $H$ such that $t=h s h^{\prime}$, that is only if $t$ belongs to the double coset modulo $H$ of $s: H_{s}=\left\{s, s^{-1}, s a^{n}, s^{-1} a^{n}, b s, b s^{-1}, b s a^{n}, b s^{-1} a^{n}\right\}$; then $\chi_{s} \odot \chi_{t}$ belongs to the subspace spanned by $\left\{\chi_{r}, r \in H_{s}\right\}$.

Proposition 6.1.1. The $C^{*}$-algebra $\widehat{K D(n)}$ decomposes as an algebra as the direct sum of $\mathbb{C} \chi_{h}$ for $h \in H$ and of $\mathbb{C} H_{a^{k}}$ for $k$ odd integer in $\{1, \ldots, n-1\}$, with

$$
H_{a^{k}}=\left\{a^{k}, a^{2 n-k}, a^{n+k}, a^{n-k}, b a^{k}, b a^{2 n-k}, b a^{n+k}, b a^{n-k}\right\} .
$$

Furthermore, each non trivial block $\mathbb{C} H_{a^{k}}$ of $\widehat{K D(n)}$ is isomorphic to $\mathbb{C} H_{a}$ in $\widehat{K D(3)}$.

Proof. The $H_{a^{k}}$ are disjoints: indeed $k$ (resp. $n-k$ ) is odd (resp. even) and strictly lower than $n$, and $2 n-k$ (resp. $n+k$ ) is odd (resp. even) and strictly larger than $n$. Therefore, the blocks $\mathbb{C} H_{a^{k}}$ and the $\mathbb{C} \chi_{h}$ are in direct sum and by dimension, they span $\widehat{K D(n)}$.

Each non trivial block $\mathbb{C} H_{a^{k}}$ of $\widehat{K D(n)}$ is stable under product; in fact, it is trivially isomorphic to $\mathbb{C} H_{a}$ in $\widehat{K D(3)}$ because the relations between the eight elements of $H_{s}$ do not depend on $s=a^{k}$. From previous considerations, products of elements in different blocs are zero. Putting everything together, they form a (non minimal) matrix block decomposition of $\widehat{K D(n)}$.

We now define eight matrix units in each non trivial block $\mathbb{C} H_{a^{k}}$. Those definitions are easily suggested and checked by computer exploration in $K D(3)$.

Definition 6.1.1. For any odd integer $k$ in $\{1, \ldots, n-1\}$, set:

$$
\begin{aligned}
E_{1,1}^{k} & =\chi_{b a^{n+k}}+\chi_{b a^{k}} \\
E_{2,2}^{k} & =\chi_{b a^{2 n-k}}+\chi_{b a^{n-k}} \\
E_{1,2}^{k} & =-\frac{1}{2} \mathrm{i}\left(-\chi_{\mathrm{a}^{\mathrm{k}}}+\chi_{\mathrm{a}^{2 \mathrm{n}-\mathrm{k}}}+\chi_{\mathrm{a}^{\mathrm{n}+\mathrm{k}}}-\chi_{\mathrm{a}^{\mathrm{n}-\mathrm{k}}}\right)+\frac{1}{2}\left(\chi_{\mathrm{ba}^{\mathrm{k}}}+\chi_{\mathrm{ba}^{2 \mathrm{n}-\mathrm{k}}}-\chi_{\mathrm{ba}^{\mathrm{n}+\mathrm{k}}}-\chi_{\mathrm{ba}^{\mathrm{n}-\mathrm{k}}}\right) \\
E_{2,1}^{k} & =\frac{1}{2} \mathrm{i}\left(-\chi_{\mathrm{a}^{\mathrm{k}}}+\chi_{\mathrm{a}^{2 \mathrm{n}-\mathrm{k}}}+\chi_{\mathrm{a}^{\mathrm{n}+\mathrm{k}}}-\chi_{\mathrm{a}^{\mathrm{n}-\mathrm{k}}}\right)+\frac{1}{2}\left(\chi_{\mathrm{ba}^{\mathrm{k}}}+\chi_{\mathrm{ba}^{2 \mathrm{n}-\mathrm{k}}}-\chi_{\mathrm{ba}^{\mathrm{n}+\mathrm{k}}}-\chi_{\mathrm{ba}^{\mathrm{n}-\mathrm{k}}}\right) \\
E_{1,1}^{n-k} & =\chi_{a^{n-k}}+\chi_{a^{2 n-k}} \\
E_{2,2}^{n-k} & =\chi_{a^{k}}+\chi_{a^{n+k}} \\
E_{1,2}^{n-k} & =\frac{1}{2}\left(-\chi_{a^{k}}-\chi_{a^{2 n-k}}+\chi_{a^{n+k}}+\chi_{a^{n-k}}\right)+\frac{1}{2} \mathrm{i}\left(\chi_{\mathrm{ba}^{\mathrm{k}}}-\chi_{\mathrm{ba}^{2 \mathrm{n}-\mathrm{k}}}-\chi_{\mathrm{ba}^{\mathrm{n}+\mathrm{k}}}+\chi_{\mathrm{ba}^{\mathrm{n}-\mathrm{k}}}\right) \\
E_{2,1}^{n-k} & =\frac{1}{2}\left(-\chi_{a^{k}}-\chi_{a^{2 n-k}}+\chi_{a^{n+k}}+\chi_{a^{n-k}}\right)-\frac{1}{2} \mathrm{i}\left(\chi_{\mathrm{ba}^{\mathrm{k}}}-\chi_{\mathrm{ba}^{2 \mathrm{n}-\mathrm{k}}}-\chi_{\mathrm{ba}^{\mathrm{n}+\mathrm{k}}}+\chi_{\mathrm{ba}^{\mathrm{n}-\mathrm{k}}}\right)
\end{aligned}
$$

6.1.2. The Kac algebra isomorphism $\psi$ from $K D(n)$ to its dual. Using definition 6.1.1, we can construct an isomorphism $\psi$ from $K D(n)$ to its dual that preserves the involution and the trace. As we noticed just before, we can check others properties of $\psi$ on computer for $n=3$ to obtain the following proposition : 
Proposition 6.1.2. The application $\psi$ defined by

$$
\begin{aligned}
& \psi\left(e_{1}\right)=\chi_{1}, \quad \psi\left(e_{2}\right)=\chi_{a^{n}}, \quad \psi\left(e_{3}\right)=\chi_{b a^{n}}, \quad \psi\left(e_{4}\right)=\chi_{b}, \\
& \psi\left(r_{i, j}^{k}\right)=E_{i, j}^{k}, \quad \psi\left(e_{i, j}^{n-k}\right)=E_{i, j}^{n-k},
\end{aligned}
$$

for $k$ odd in $\{1, \ldots, n-1\}, i=1,2$, and $j=1,2$ extends by linearity into a $C^{*}$-algebra from $K D(2 m+1)$ to $K D \widehat{(2 m+1)}$ which preserves the trace $t r$.

Proof of Theorem 6.1. In A.8, we calculate explicitly $\psi(\lambda(a))$ and $\psi(\lambda(b))$, and check that the corresponding coproducts are preserved by $\psi$. It follows that the isomorphism $\psi$ is a $C^{*}$-algebra isomorphism which preserves the trace and the coproducts of the generators of $K D(n)$. It therefore also preserves the coinvolution (see 2.8.1), and is a Kac algebra isomorphism from $K D(n)$ to its dual.

6.2. The symmetric Kac subalgebras. The lattice $1(K D(n))$ contains the algebra $J_{0}$ of the intrinsic group of $K D(n)$ (see [5.4.3) and its Kac subalgebras:

- $J_{0}=\mathbb{C}\left(e_{1}+q_{1}\right) \oplus \mathbb{C}\left(e_{2}+q_{2}\right) \oplus \mathbb{C}\left(e_{3}+p_{2}\right) \oplus \mathbb{C}\left(e_{4}+p_{1}\right)$ spanned by $1, \lambda\left(a^{n}\right), \lambda(b)$ and $\lambda\left(b a^{n}\right)$

- $I_{0}=\mathbb{C}\left(e_{1}+e_{2}+q_{1}+q_{2}\right) \oplus \mathbb{C}\left(e_{3}+e_{4}+p_{1}+p_{2}\right)$ spanned by 1 and $\lambda\left(a^{n}\right)$;

- $I_{1}=\mathbb{C}\left(e_{1}+e_{3}+q_{1}+p_{2}\right) \oplus \mathbb{C}\left(e_{2}+e_{4}+p_{1}+q_{2}\right)$ spanned by 1 and $\lambda\left(b a^{n}\right)$;

- $I_{2}=\mathbb{C}\left(e_{1}+e_{4}+p_{1}+q_{1}\right) \oplus \mathbb{C}\left(e_{2}+e_{3}+p_{2}+q_{2}\right)$ spanned by 1 and $\lambda(b)$.

Using 2.8.6, the only coideal subalgebras of dimension 2 of $K D(n)$ are $I_{0}, I_{1}$, and $I_{2}$.

Since $K D(n)$ is self-dual, we can describe the principal graph of an inclusion $N_{2} \subset N_{2} \rtimes J$ as soon as $\delta(J)$ is known after identification of $K D(n)$ and its dual. Considering the Bratteli diagram of $I_{i} \subset K D(n)$ (with $\left.i=0,1,2\right)$ shows that the inclusion $N_{2} \subset N_{2} \rtimes \delta^{-1}\left(I_{0}\right)$ is the only one of depth 2 . Therefore, by 2.8.3. $\delta\left(K_{2}\right)$ has to be identified with $I_{0}$. Then, the principal graph of $N_{2} \subset N_{2} \rtimes K_{3}$ and of $N_{2} \subset N_{2} \rtimes K_{4}$ is $D_{2 n} / \mathbb{Z}_{2}$ (see 4.2).

Since $J_{0}$ contains the three coideal subalgebras of dimension $2, \delta\left(K_{1}\right)$ is $J_{0}$, the intersection of the three coideal subalgebras of dimension $2 n$. The graph of the inclusion $N_{2} \subset N_{2} \rtimes K_{1}$ is therefore $D_{n} / \mathbb{Z}_{2}$ (see 4.1). Furthermore, using [HS96], the inclusion is of the form $R \rtimes \mathbb{Z}_{2} \subset R \rtimes G$, where $G$ is the semi-direct product of an abelian group of order $n$ with $\mathbb{Z}_{2}$.

\subsection{The other coideal subalgebras of dimension $2 n$ : $K_{3}$ and $K_{4}$.}

\subsubsection{The coideal subalgebras $K_{3}$ and $K_{4}$ are isomorphic.}

Proposition 6.3.1. Let $n$ be odd. Then, the automorphism $\Theta^{\prime}$ of $K D(n)$ (see Proposition 5.5.2) exchanges $e_{3}$ and $e_{4}$. Therefore, the coideal subalgebras $K_{3}=I\left(e_{1}+e_{3}\right)$ and $K_{4}=I\left(e_{1}+e_{4}\right)$ of $K D(n)$ are exchanged by $\Theta^{\prime}$ and thus isomorphic.

Proof. Using A.2, and dropping the $\lambda$ 's for clarity, we have:

$$
\Theta^{\prime}\left(e_{1}+e_{3}\right)=\frac{1}{2 n} \sum_{k=0}^{n-1} a^{2 k}+b a^{2 k+1+n}-\frac{1}{4 n} \sum_{k=0}^{n-1}\left[a^{2 k}-a^{-2 k}+b a^{2 k+1}-b a^{-2 k-1}\right]\left(1+a^{n}\right) .
$$

Since $n$ is odd, the first sum is $e_{1}+e_{4}$, while the second is obviously null.

6.3.2. Decomposition of $K_{3}$ and $K_{4}$. From the expressions of the coproducts of the projections $e_{1}+e_{3}$ and $e_{1}+e_{4}$ for $n$ odd, one deduces:

$$
K_{3}=\mathbb{C}\left(e_{1}+e_{3}\right) \oplus \mathbb{C}\left(e_{2}+e_{4}\right) \oplus \bigoplus_{j=1, j \text { odd }}^{n-1} K_{3}^{j},
$$




$$
K_{4}=\mathbb{C}\left(e_{1}+e_{4}\right) \oplus \mathbb{C}\left(e_{2}+e_{3}\right) \oplus \bigoplus_{j=1, j \text { odd }}^{n-1} K_{4}^{j},
$$

where each $K_{3}^{j}$ and $K_{4}^{j}$ is a factor $M_{2}(\mathbb{C})$ with matrix units:

$$
\left(e_{1,1}^{j}+r_{1,1}^{n-j}\right),\left(e_{2,2}^{j}+r_{2,2}^{n-j}\right),\left(e_{1,2}^{j}+r_{1,2}^{n-j}\right), \text { and }\left(e_{2,1}^{j}+r_{2,1}^{n-j}\right),
$$

and

$$
\left(e_{1,1}^{j}+r_{2,2}^{n-j}\right),\left(e_{2,2}^{j}+r_{1,1}^{n-j}\right),\left(e_{1,2}^{j}-r_{2,1}^{n-j}\right), \text { and }\left(e_{2,1}^{j}-r_{1,2}^{n-j}\right),
$$

respectively. From $2.4 .2(5), I_{1}$ is contained in $K_{3}$ and $I_{2}$ in $K_{4}$.

6.4. The coideal subalgebra $K_{1}=I\left(e_{1}+e_{2}+e_{3}+e_{4}\right)$ of dimension $n$.

Proposition 6.4. For $n=2 m+1$, the matrix structure of $K_{1}=I\left(e_{1}+e_{2}+e_{3}+e_{4}\right)=$ $K_{2} \cap K_{3} \cap K_{4}$ is given by

$$
K_{1}=\mathbb{C}\left(e_{1}+e_{2}+e_{3}+e_{4}\right) \oplus \bigoplus_{j=1, j \text { odd }}^{n-1} \mathbb{C}\left(r_{1,1}^{j}+e_{1,1}^{n-j}\right) \oplus \mathbb{C}\left(r_{2,2}^{j}+e_{2,2}^{n-j}\right) .
$$

In $K_{2} \equiv D_{n}$ it is the subalgebra of constant functions on the right cosets w.r.t. $\{1, \beta\}$. Using the notations of 5.7, the matrix structure can be written as:

$$
K_{1}=\bigoplus_{j=0, \ldots, n-1} \mathbb{C}\left(\chi_{\alpha^{j}}+\chi_{\alpha^{j} \beta}\right) .
$$

Each coideal subalgebra $I$ of $K_{1}$ is the subalgebra of constant functions on the right cosets of some subgroup of $D_{n}$ containing $\{1, \beta\}$. Such a subgroup is a dihedral group generated by $\beta$ and $\alpha^{k}$ for some $k$ divisor of $n$. Then, the Jones projection of $I$ is $e_{1}+e_{2}+e_{3}+e_{4}+$ $\sum_{h=1}^{(n-1) / 2 k} e_{1,1}^{2 h k}+e_{2,2}^{2 h k}+r_{1,1}^{n-2 h k}+r_{2,2}^{n-2 h k}$.

Proof. Straightforward using 5.9 and Appendix $\mathbf{A . 5 . 2}$

6.5. The coideal subalgebras of dimension dividing $n$. From 5.10 , the other coideal subalgebras of dimension dividing $2 n$ are contained in one of the coideal subalgebras of dimension $2 n$. We now show a more precise result for those of dimension dividing $n$ (or equivalently of odd dimension).

Proposition 6.5. When $n$ is odd, the coideal subalgebras of odd dimension of $K D(n)$ are those of $K_{2}$; in particular, they are isomorphic to the algebra of functions on $D_{n}$ which are constant on right cosets w.r.t. one of its subgroups. There are exactly $n$ coideal subalgebras of dimension $n$, whose Jones projections are given in 5.8 .

Proof. Let $I$ be a coideal subalgebra of odd dimension $k$. Then, $k$ is an odd divisor of $2 n$. Set $k^{\prime}=2 n / k$ which is even. From 5.10, either $I$ is contained in $K_{1}$ (and therefore in $K_{2}$, or its Jones projection is of the form $e_{1}+e_{i}+s$, where $i=2,3$ or 4 and $s$ is a projection of trace $1 / k-1 / 2 n=\left(k^{\prime}-1\right) / 2 n$ distinct from $e_{j}+e_{j^{\prime}}$. However, in $K_{3}$ (resp. $\left.K_{4}\right)$, and except for $e_{2}+e_{4}$ (resp. $e_{2}+e_{3}$ ), the minimal projections are of trace $1 / n$, so it is not possible to combine them to get a projection of trace $\left(k^{\prime}-1\right) / 2 n$ with $k^{\prime}$ even. Therefore, $p_{I}$ is of the form $e_{1}+e_{2}+s$, and by 2.4.2 (5), $I \subset K_{2}$. 
6.6. The coideal subalgebras of $K_{3}$ and $K_{4}$. Since $K_{3}$ and $K_{4}$ are isomorphic by $\Theta^{\prime}$, their lattice of coideal subalgebras are isomorphic. We focus on the description of that of $K_{4}$. From 6.5, the coideal subalgebras of odd dimension of $K_{4}$ are those of $K_{1}$, and therefore contained in $K_{2}$. For the others, we have the following partial description.

Proposition 6.6. Let $n=2 m+1$, and $k$ a divisor of $n=k d$. Let $B_{2 d}$ be the subgroup generated by $a^{2 d}$ and $b$ as in Proposition 5.11. Then,

$$
e_{1}+e_{4}+\sum_{j=1}^{d-1} p_{1,1}^{j k}=\frac{1}{2 k} \sum_{g \in B_{2 d}} \lambda(g)
$$

is the Jones projection of a coideal subalgebra of dimension $2 k$ of $K_{4}$, image by $\varphi_{d}$ of the $K_{4}$ of $K D(k)$.

In general, every coideal subalgebra of dimension $2 k$ of $K_{4}$ contains $I_{2}$. Its Jones projection is of the form:

$$
e_{1}+e_{4}+\sum_{t \in T} p_{1,1}^{2 t}+p_{1,1}^{n-2 t}
$$

where $T$ is a subset of $\{1,2, \ldots, m\}$ of size $\frac{1}{2}(d-1)$. In particular, the Jones projection for $B_{2 d}$ above is obtained when $T$ is the set of multiples of $k$ in $\{1, \ldots, m\}$.

Proof. The first part is the third item of Proposition 5.11.

Let $k$ be a non trivial divisor of $n$ and $L$ a coideal subalgebra of dimension $2 k$ of $K_{4}$. Then, $\delta(L)$ is of dimension $2 d$, and by 5.10 it is contained in exactly one of $K_{2}, K_{3}$, and $K_{4}$. Therefore, $L$ contains exactly one of $I_{0}, I_{1}$, and $I_{2}$, and from the expressions of $p_{I_{i}}$, $i=0,1,2$ (see 6.2), this is necessarily $I_{2}$.

The Jones projection $p_{L}$ of $L$ is of trace $1 / 2 k$, dominates $p_{K_{4}}=e_{1}+e_{4}$, and is dominated by $p_{I_{2}}=e_{1}+e_{4}+q_{1}+p_{1}$. Using the matrix units of $K_{4}$ (see 6.3.2), it has to be of the given form.

6.7. The coideal subalgebras of dimension 4. The following proposition was suggested by the computer exploration of Appendix B.5.3, where we use the explicit isomorphism between $K D(n)$ and its dual (see 6.1) to derive the Jones projections of the coideal subalgebras of dimension 4 from the coideal subalgebras of dimension $n$ of $K_{2}$.

Proposition 6.7. When $n$ is odd, there are $n$ coideal subalgebras $\left(J_{k}\right)_{k=0, \ldots, n-1}$ of $d i$ mension 4 in $K D(n)$. The Jones projection of $J_{k}$ is $p_{J_{k}}=e_{1}+\sum_{j=1}^{m} q\left(0, \frac{2 k j \pi}{n}, 2 j\right)$. The principal graph of the inclusions $N_{1} \subset N_{1} \rtimes \delta\left(J_{k}\right)$ and $N_{2} \subset N_{2} \rtimes J_{k}$ are respectively $D_{n} / \mathbb{Z}_{2}$ (see 4.1) and $D_{2 n+2}^{(1)}$.

Proof. By self-duality, the coideal subalgebras of dimension 4 are mapped by $\delta$ to the coideal subalgebras of dimension $n$ of $K_{2}$, and there are $n$ of them (see 5.8). The Jones projections $p_{J_{k}}$ are given in Proposition A.4 together with the central projections of $J_{k}$; the principal graph of $N_{1} \subset N_{1} \rtimes \delta\left(J_{k}\right)$ follows. The principal graph of $N_{2} \subset N_{2} \rtimes J_{k}$ follows from 5.8 .

6.8. The lattice $1(K D(n))$. The following theorem summarizes the results of this section, and describe most, if not all (see Conjecture 6.8), of the lattice $1(K D(n))$ of coideal subalgebras of $K D(n)$.

Theorem 6.8. When $n$ is odd, the lattice $1(K D(n))$ is self-dual. It has:

- 3 coideal subalgebras of dimension $2: I_{0}, I_{1}$, and $I_{2}$, contained in the intrinsic group $J_{0}$

- 3 coideal subalgebras of dimension $2 n$ :

- $K_{2}$, isomorphic to $\mathcal{L}^{\infty}\left(D_{n}\right)$; 
- $K_{3}$ and $K_{4}$, isomorphic via $\Theta^{\prime}$, but non Kac subalgebras;

- $n$ coideal subalgebras of dimension $n$ contained in $K_{2}: K_{1}=I\left(e_{1}+e_{2}+e_{3}+e_{4}\right)$, and, for $j=0, \ldots, m-1, I\left(e_{1}+e_{2}+r_{1,1}^{2 j+1}\right)$ and $I\left(e_{1}+e_{2}+r_{2,2}^{2 j+1}\right)$;

- $n$ coideal subalgebras of dimension 4 :

$$
I\left(e_{1}+\sum_{j=1}^{m} q\left(0, \frac{2 k j \pi}{n}, 2 j\right)\right), \quad \text { for } k=0, \ldots, n-1 .
$$

When $n$ is not prime, $\mathrm{l}(K D(n))$ contains further:

- the coideal subalgebras of odd dimension dividing strictly $n$, contained in $K_{2}$, and associated to the dihedral subgroups of $D_{n}$;

- the coideal subalgebras of dimension a multiple of 4 , image of the previous ones by $\delta$;

- the coideal subalgebras of even dimension dividing strictly $2 n$ :

- the coideal subalgebras of $K_{2}$ corresponding to the subgroups of $\langle\alpha\rangle$ in $D_{n}$;

- the coideal subalgebras of $K_{3}$ containing $I_{1}$; they are the images by $\Theta^{\prime}$ of those of $K_{4}$ containing $I_{2}$ below;

- coideal subalgebras of $K_{4}$ containing $I_{2}$ :

* for each $k$ non trivial divisor of $n=k d$, the image of the coideal subalgebra $K_{4}$ of dimension $2 k$ of $K D(k)$ by the embedding $\varphi_{d}$;

* a set $\mathcal{I}$ of other coideal subalgebras, controlled by Proposition 6.6, and empty for $n \leq 51$.

Proof. Follows from Theorem 6.1, and Propositions 5.10, 6.3.1, 6.4, 6.5, 6.6, and 6.7, The emptyness of $\mathcal{I}$ for $n \leq 51$ was checked on computer, using that, by Proposition 6.6) there are only finitely many possible Jones projections for coideal subalgebras in $\mathcal{I}$. This check can be further reduced by using $\delta$ which exchanges coideal subalgebras of dimension $2 k$ and $2 d$.

Corollary 6.8. For $n$ odd prime, the non trivial coideal subalgebras of $K D(n)$ are of dimension 2, 4, $n$, and $2 n$ and the graph of the lattice of coideal subalgebras of $K D(n)$ is similar to Figure 8 with $n$ coideal subalgebras of dimension 4 and $n$.

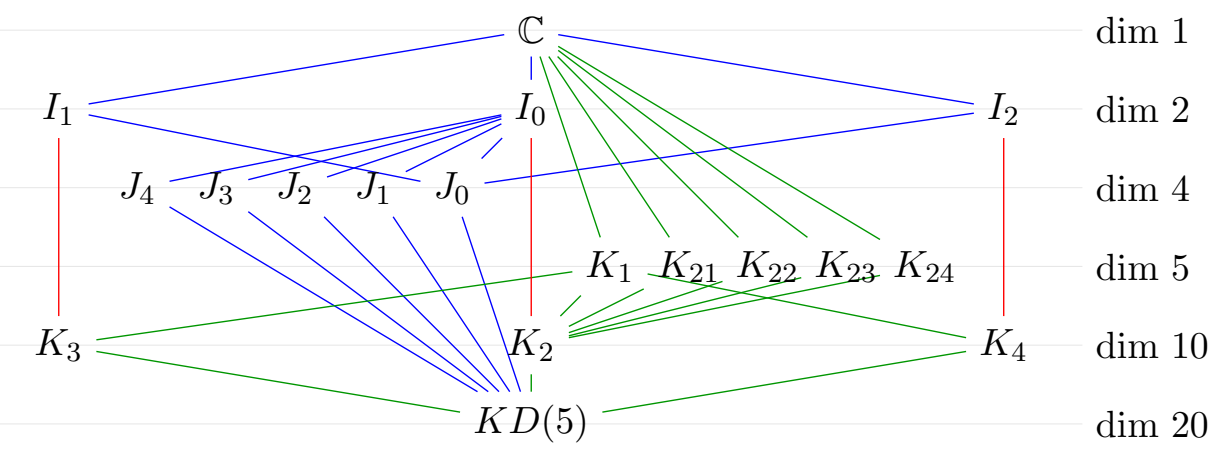

Figure 8. The lattice of coideal subalgebras of $K D(5)$

The computer exploration results mentioned in Theorem 6.8 suggests right away the following conjecture when $n$ is not prime.

Conjecture 6.8. Let $n$ be odd. Then, the description of the lattice $1(K D(n))$ of Theorem 6.8 is complete: $\mathcal{I}$ is empty. 


\subsection{The Kac algebra $K D(3)$ of dimension 12.}

6.9.1. The lattice $1(K D(3))$. In this section, we describe the lattice of $K D(3)$ in detail to illustrate Corollary 6.8.

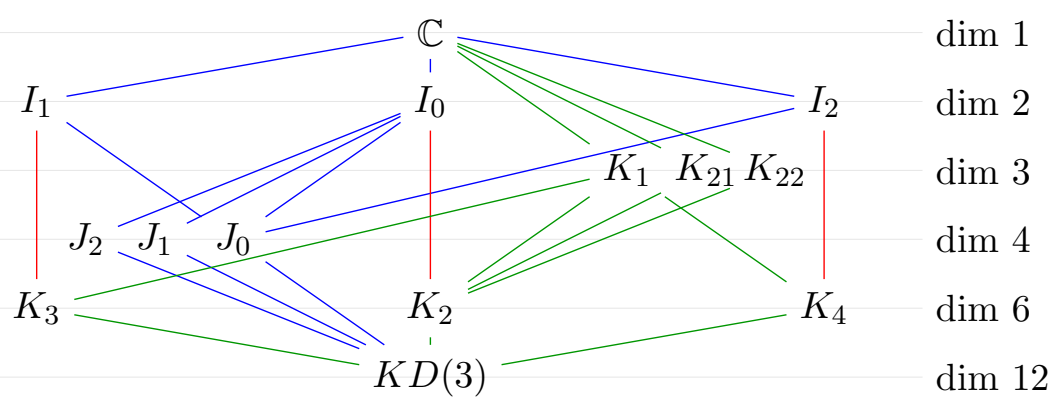

Figure 9. The lattice of coideal subalgebras of $K D(3)$

Proposition 6.9.1. In $K D(3)$,

- The coideal subalgebras of dimension 2 are $I_{0}, I_{1}$, and $I_{2}$;

- The coideal subalgebras of dimension 3 are contained in $K_{2}$; they are the algebras of functions constant w.r.t. the three subgroups of order 2 :

$$
\begin{aligned}
K_{1} & =\mathbb{C}\left(e_{1}+e_{2}+e_{3}+e_{4}\right) \oplus \mathbb{C}\left(r_{1,1}^{1}+e_{1,1}^{2}\right) \oplus \mathbb{C}\left(r_{2,2}^{1}+e_{2,2}^{2}\right) ; \\
K_{21} & =\mathbb{C}\left(e_{1}+e_{2}+r_{1,1}^{1}\right) \oplus \mathbb{C}\left(e_{3}+e_{4}+e_{2,2}^{2}\right) \oplus \mathbb{C}\left(r_{2,2}^{1}+e_{1,1}^{2}\right) ; \\
K_{22} & =\mathbb{C}\left(e_{1}+e_{2}+r_{2,2}^{1}\right) \oplus \mathbb{C}\left(e_{3}+e_{4}+e_{1,1}^{2}\right) \oplus \mathbb{C}\left(r_{1,1}^{1}+e_{2,2}^{2}\right) .
\end{aligned}
$$

- The coideal subalgebras of dimension 4 , images of those of dimension 3 by $\delta$, are: $J_{0}=\mathbb{C}\left(e_{1}+q_{1}\right) \oplus \mathbb{C}\left(e_{2}+q_{2}\right) \oplus \mathbb{C}\left(e_{3}+p_{2}\right) \oplus \mathbb{C}\left(e_{4}+p_{1}\right) ;$

$J_{1}=\mathbb{C}\left(e_{1}+q\left(0, \frac{2 \pi}{3}, 2\right)\right) \oplus \mathbb{C}\left(e_{2}+q\left(0, \frac{5 \pi}{3}, 2\right)\right) \oplus \mathbb{C}\left(e_{3}+q\left(\frac{\pi}{3}, 0,1\right)\right) \oplus \mathbb{C}\left(e_{4}+q\left(-\frac{\pi}{3}, \pi, 1\right)\right) ;$ $J_{2}=\mathbb{C}\left(e_{1}+q\left(0, \frac{4 \pi}{3}, 2\right)\right) \oplus \mathbb{C}\left(e_{2}+q\left(0, \frac{\pi}{3}, 2\right)\right) \oplus \mathbb{C}\left(e_{3}+q\left(-\frac{\pi}{3}, 0,1\right)\right) \oplus \mathbb{C}\left(e_{4}+q\left(\frac{\pi}{3}, \pi, 1\right)\right)$.

- The three coideal subalgebras of dimension 6 are $K_{2}, K_{3}$, and $K_{4}$.

With the notations of 母, the inclusion $N_{2} \subset N_{2} \rtimes J$ has for principal graph

- $D_{8}^{(1)}$ for $J=J_{1}$ or $J_{2}$.

- $A_{5}$ for $J=K_{1}, K_{21}$ or $K_{22}$,

- $D_{6} / \mathbb{Z}_{2}$ for $J=K_{3}$ or $K_{4}$.

For $J=I_{0}, I_{1}, I_{2}, J_{0}$ or $K_{2}$, it is of depth 2 .

The lattice of coideal subalgebras de $K D(3)$ is as given in Figure 9 .

6.9.2. Realization of $N_{2} \subset N_{2} \rtimes J_{1}$ by composition of subfactors. As in 3.4, the inclusion $N_{2} \subset N_{2} \rtimes J_{i}$ of principal graph $D_{8}^{(1)}$ can be interpreted as $M^{\left(\alpha, \mathbb{Z}_{2}\right)} \subset M \rtimes_{\beta} \mathbb{Z}_{2}$ (see also Pop90).

Proposition 6.9.2. The inclusion $N_{2} \subset N_{2} \rtimes J_{1}$, which is of principal graph $D_{8}^{(1)}$, can be put under the form $M^{\left(\alpha, \mathbb{Z}_{2}\right)} \subset M \rtimes_{\beta} \mathbb{Z}_{2}$ as follows. Take the following basis of $J_{1}$ :

$B_{1}=e_{1}+q\left(0, \frac{2 \pi}{3}, 2\right), B_{2}=e_{2}+q\left(0, \frac{5 \pi}{3}, 2\right), B_{3}=e_{3}+q\left(\frac{\pi}{3}, 0,1\right), B_{4}=e_{4}+q\left(-\frac{\pi}{3}, \pi, 1\right)$, and set $v=B_{1}-B_{2}+B_{3}-B_{4}$. Recall that $\lambda\left(a^{3}\right)=B_{1}+B_{2}-B_{3}-B_{4}$. 
Set $M=N_{2} \rtimes I_{0}$, and let $\alpha$ be the automorphism of $M$ which fixes $N_{2}$ and changes $\lambda\left(a^{3}\right)$ into its opposite (this is the dual action of $\mathbb{Z}_{2}$ ) and $\beta=\operatorname{Ad} v$. Then, $\alpha$ and $\beta$ are involutive automorphisms of $M$ such that the period of $\beta \alpha$ is 6 , while $M^{\alpha}=N_{2}$ and $N_{2} \rtimes J_{1}=M \rtimes_{\beta} \mathbb{Z}_{2}$.

Proof. As in 2.1.2 (see [Dav05]), we identify $N_{3}$ and $N_{2} \rtimes K D(3)$. Therefore, for all $x \in N_{2}$, one has $\beta(x)=v x v^{*}=\left(v_{(1)} \triangleright x\right) v_{(2)} v^{*}$. From $\Delta(v)=v \otimes\left(B_{1}-B_{2}\right)+w \otimes\left(B_{3}-B_{4}\right)$, (with $\left.w=\left(e_{1}+q\left(0,-\frac{2 \pi}{3}, 2\right)\right)-\left(e_{2}+q\left(0, \frac{\pi}{3}, 2\right)\right)+\left(e_{3}+q\left(-\frac{\pi}{3}, 0,1\right)\right)-\left(e_{4}+q\left(\frac{\pi}{3}, \pi, 1\right)\right)\right)$, we get, for $x \in N_{2}$,

$$
\beta(x)=(v \triangleright x)\left(B_{1}+B_{2}\right)+(w \triangleright x)\left(B_{3}+B_{4}\right),
$$

and deduce that $\beta$ normalizes $M$. By a straightforward calculation, we obtain $(w v)^{3}=$ $(v w)^{3}=1$ and $(\beta \alpha)^{6}=\mathrm{id}$. Then, $N_{2} \rtimes J_{1}$ is indeed the cross product of $M$ by $\beta$, since $\lambda\left(a^{3}\right)$ and $v$ generate the subalgebra $J_{1}$.

6.10. The algebra $K D(9)$ of dimension 36. We illustrate, on $K D(9)$, Theorem 6.8 for $n$ not prime.

Corollary 6.10. The lattice of coideal subalgebras of $K D(9)$ is given by Figure 10. Namely:

- The coideal subalgebras of dimension 2 are $I_{0}, I_{1}$ et $I_{2}$.

- The coideal subalgebras of dimension 3 are contained in $K_{2}$ :

- The coideal subalgebra of constant functions modulo $\left\langle\alpha^{3}, \beta\right\rangle$ is $L_{0}=I\left(e_{1}+e_{2}+e_{3}+e_{4}+r_{1,1}^{3}+r_{2,2}^{3}+e_{1,1}^{6}+e_{2,2}^{6}\right)$

- The coideal subalgebra of constant functions modulo $\left\langle\alpha^{3}, \beta \alpha\right\rangle$ is $L_{1}=I\left(e_{1}+e_{2}+r_{2,2}^{1}+r_{1,1}^{5}+e_{1,1}^{6}+e_{2,2}^{6}+r_{2,2}^{7}\right)$

- The coideal subalgebra of constant functions modulo $\left\langle\alpha^{3}, \beta \alpha^{2}\right\rangle$ is $L_{2}=I\left(e_{1}+e_{2}+r_{1,1}^{1}+r_{2,2}^{5}+e_{1,1}^{6}+e_{2,2}^{6}+r_{1,1}^{7}\right)$

- The nine coideal subalgebras of dimension 4 are the $J_{k}=I\left(e_{1}+\sum_{j=1}^{4} q\left(0, \frac{2 k j \pi}{9}, 2 j\right)\right)$, for $k=0, \ldots, 8$;

- The coideal subalgebras of dimension 6 are:

- The Kac subalgebra of constant functions modulo $\left\langle\alpha^{3}>\right.$ in $K_{2}$ : $M_{2}=I\left(e_{1}+e_{2}+e_{1,1}^{6}+e_{2,2}^{6}\right)$ which contains all the $L_{i}, i=0,1,2$;

- The coideal subalgebra $M_{3}=I\left(e_{1}+e_{3}+p_{2,2}^{3}+p_{1,1}^{6}\right)$ contained in $K_{3}$;

- The coideal subalgebra $M_{4}=I\left(e_{1}+e_{4}+p_{1,1}^{3}+p_{1,1}^{6}\right)$ contained in $K_{4}$.

- The nine coideal subalgebras of dimension 9 are contained in $K_{2}$ :

$K_{1}=I\left(e_{1}+e_{2}+e_{3}+e_{4}\right), K_{21}=e_{1}+e_{2}+r_{1,1}^{3}$, and $K_{22}=e_{1}+e_{2}+r_{2,2}^{3}$ whose intersection is $L_{0}$;

$K_{23}=e_{1}+e_{2}+r_{2,2}^{1}, K_{24}=e_{1}+e_{2}+r_{1,1}^{5}$, and $K_{25}=e_{1}+e_{2}+r_{2,2}^{7}$ whose intersection is $L_{1}$;

$K_{23}=e_{1}+e_{2}+r_{2,2}^{1}, K_{24}=e_{1}+e_{2}+r_{1,1}^{5}$, and $K_{25}=e_{1}+e_{2}+r_{2,2}^{7}$ whose intersection is $L_{1}$;

$K_{26}=e_{1}+e_{2}+r_{1,1}^{1}, K_{27}=e_{1}+e_{2}+r_{2,2}^{5}$, and $K_{28}=e_{1}+e_{2}+r_{1,1}^{7}$ whose intersection is $L_{2}$.

- The three coideal subalgebras of dimension 12 , images by $\delta$ of $L_{i}, i=0,1,2$. Their Jones projections are $e_{1}+p_{1,1}^{6}$ for $\delta\left(L_{0}\right)$, and $\left.e_{1}+q\left(0, \frac{2 \pi}{3}, 6\right)\right)$ and $\left.e_{1}+q\left(0, \frac{4 \pi}{3}, 6\right)\right)$ for the two others.

- The three coideal subalgebras of dimension 18 are $K_{2}, K_{3}$, and $K_{4}$.

Proof. Most of this proposition follows from Theorem 6.8. To derive the formulas for the Jones projections of $\delta\left(L_{i}\right)$, we noticed that they all contain $M_{2}$ as well as three $J_{k}$ 's, and looked on computer through the projectors of trace $1 / 12$ which dominates the projections of those coideal subalgebras. Since $L_{0}$ is contained in $K_{1}, \delta\left(L_{0}\right)$ contains $J_{0}$. 


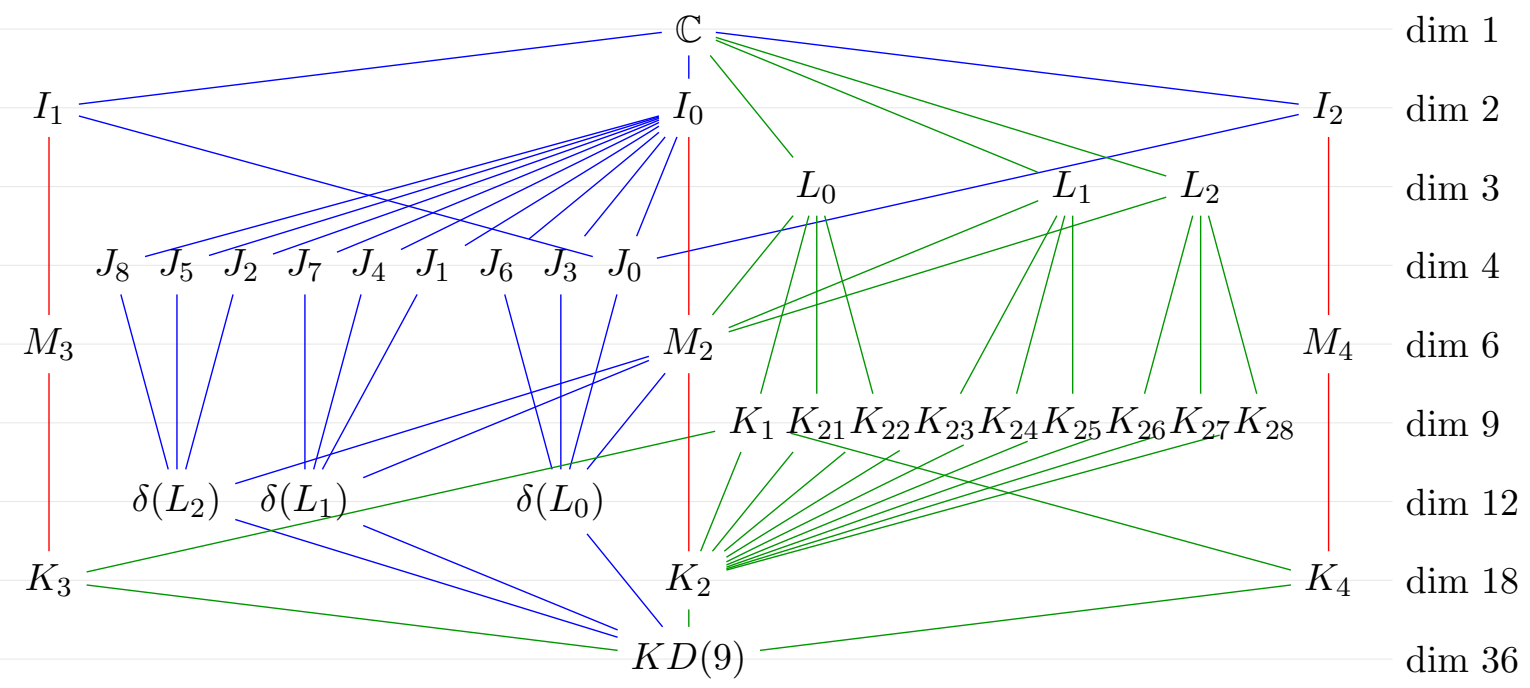

Figure 10. The lattice of coideal subalgebras of $K D(9)$

6.11. The algebra $K D(15)$ of dimension 60 . Figure 11 illustrates, on $K D(15)$, Theorem 6.8 for $n$ not the square of a prime.

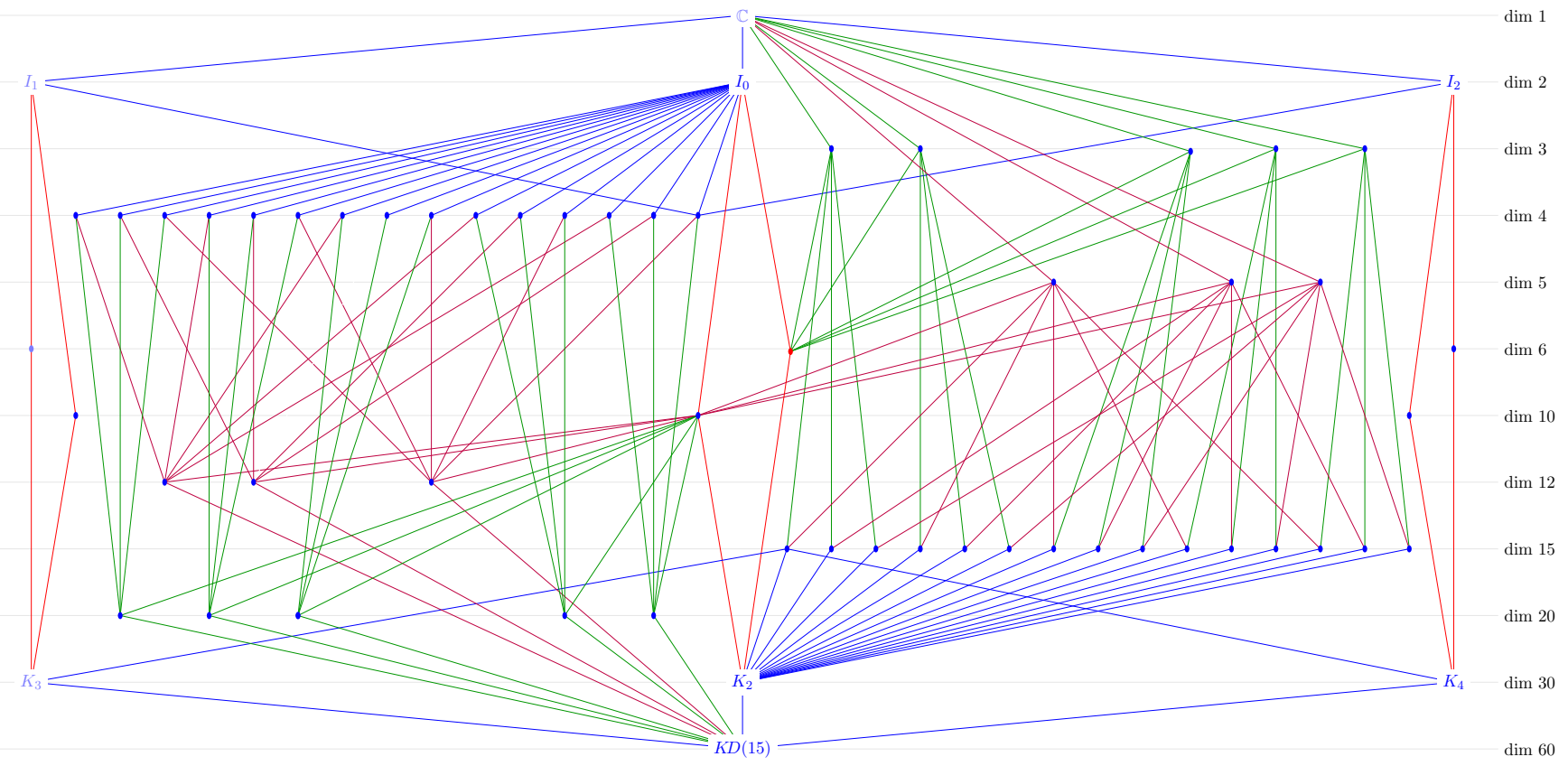

Figure 11. The lattice of coideal subalgebras of $K D(15)$ 


\section{The KaC Algebras $K D(n)$ FOR $n$ EVEN}

In this section we assume that $n$ is even: $n=2 m$. The structure of $K D(n)$ is then very different from the odd case. The intrinsic group is $D_{4}$, and the algebra $K D(2 m)$ is never self-dual. On the other hand, the coideal subalgebras $K_{3}$ and $K_{4}$ are non isomorphic Kac subalgebras. Using that $K_{2}$ is isomorphic to $L^{\infty}\left(D_{n}\right), K_{4}$ to $K D(m)$ and $K_{3}$ to $K B(m)$ (i.e. $K Q(m)$ for $m$ odd), we recover a large part of the lattice of coideal subalgebras by induction. For example the lattices of coideal subalgebras of $K D(4)$ (see 7.6) and $K D(8)$ (see 9.3.2) and a large part of the lattice of $K D(6)$ (see 7.7) can be constructed this way.

7.1. The algebra of the intrinsic group $K_{0}$. We start with the algebra $K_{0}$ of the intrinsic group of $K D(n)$. From [5.4.2 and [5.4.3, it is generated by $\lambda\left(a^{m}\right)$ and $\lambda(b)$, and isomorphic to $K D(2) \equiv \mathbb{C}\left(D_{4}\right)$.

7.1.1. The isomorphism between $K D(2)$ and $K_{0}$ using matrix units. We have seen in 5.4 .2 that the application $\varphi_{m}$ from $K D(2)$ to $K_{0}$ which sends $\lambda(b)$ to $\lambda(b)$ and $\lambda(a)$ to $\lambda\left(a^{m}\right)$ can be extended into a Kac algebra isomorphism. We now give the expression of $\varphi_{m}$ on the matrix units of $K D(2)$ (which will be marked with a ' to avoid confusion). Taking the conditions on the index $j$ modulo 4 , and using the formulas A.1 in $K D(2)$ and $K_{0}$, we obtain:

For $n=2 m=4 m^{\prime}$ :

$$
\begin{aligned}
\varphi_{m}\left(e_{1}^{\prime}\right) & =e_{1}+e_{4}+\sum_{j \equiv 0} p_{1,1}^{j}, & \varphi_{m}\left(e_{2}^{\prime}\right) & =e_{2}+e_{3}+\sum_{j \equiv 0} p_{2,2}^{j}, \\
\varphi_{m}\left(e_{3}^{\prime}\right) & =\sum_{j \equiv 2} p_{2,2}^{j}, & \varphi_{m}\left(e_{4}^{\prime}\right) & =\sum_{j \equiv 2} p_{1,1}^{j} .
\end{aligned}
$$

For $n=2 m=4 m^{\prime}+2$ :

$$
\begin{array}{ll}
\varphi_{m}\left(e_{1}^{\prime}\right)=e_{1}+\sum_{j \equiv 0} p_{1,1}^{j}, & \varphi_{m}\left(e_{2}^{\prime}\right)=e_{2}+\sum_{j \equiv 0} p_{2,2}^{j}, \\
\varphi_{m}\left(e_{3}^{\prime}\right)=e_{3}+\sum_{j \equiv 2} p_{2,2}^{j}, & \varphi_{m}\left(e_{4}^{\prime}\right)=e_{4}+\sum_{j \equiv 2} p_{1,1}^{j} .
\end{array}
$$

In both cases:

$$
\varphi_{m}\left(e_{1,2}^{\prime}\right)=\sum_{j \equiv 1} e_{1,2}^{j}+\sum_{j \equiv 3} e_{2,1}^{j} .
$$

7.1.2. The lattice $1\left(K_{0}\right)$ ). We obtain the central projections and the lattice of coideal subalgebras of $K_{0}$ by combining the results of 5.3 and 7.1.1. All the coideal subalgebras are Kac subalgebras. We keep the same naming convention for the coideal subalgebras in $K D(2)$ and their images by $\varphi_{m}$ in $K_{0}$.

The algebra $K_{0}$ contains $J_{0}=\mathbb{C}[H]$ and all its coideal subalgebras (note that by 2.4.2 (5), those are also coideal subalgebras of $\left.K_{4}\right)$ :

- $J_{0}=\mathbb{C}\left(e_{1}+e_{4}+q_{1}\right) \oplus \mathbb{C}\left(e_{2}+e_{3}+q_{2}\right) \oplus \mathbb{C} p_{2} \oplus \mathbb{C} p_{1}$;

- $I_{0}=\mathbb{C}\left(e_{1}+e_{2}+e_{3}+e_{4}+q_{1}+q_{2}\right) \oplus \mathbb{C}\left(p_{1}+p_{2}\right)$;

- $I_{1}=\mathbb{C}\left(e_{1}+e_{4}+q_{1}+p_{2}\right) \oplus \mathbb{C}\left(e_{2}+e_{3}+p_{1}+q_{2}\right)$;

- $I_{2}=\mathbb{C}\left(e_{1}+e_{4}+p_{1}+q_{1}\right) \oplus \mathbb{C}\left(e_{2}+e_{3}+p_{2}+q_{2}\right)$.

The basis of the other coideal subalgebras depends on the parity of $m$ :

For $n=4 m^{\prime}$ : The Jones projection of $K_{0}$ is $e_{1}+e_{4}+\sum_{j=1}^{m^{\prime}-1} p_{1,1}^{4 j}$. The other central projection in the same connected component of the Bratelli diagram of $K_{0} \subset$ $K D(n)$ is $e_{2}+e_{3}+\sum_{j \equiv 0} p_{2,2}^{j}$. The coideal subalgebras not contained in $J_{0}$ are:

- $I_{3}=I\left(e_{1}+e_{2}+e_{3}+e_{4}+\sum_{j \equiv 0}\left(e_{1,1}^{j}+e_{2,2}^{j}\right)+\sum_{j \equiv 1} r_{2,2}^{j}+\sum_{j \equiv 3} r_{1,1}^{j}\right)$; 
- $I_{4}=I\left(e_{1}+e_{2}+e_{3}+e_{4}+\sum_{j \equiv 0}\left(e_{1,1}^{j}+e_{2,2}^{j}\right)+\sum_{j \equiv 1} r_{1,1}^{j}+\sum_{j \equiv 3} r_{2,2}^{j}\right)$;

- $J_{20}=I\left(e_{1}+e_{2}+e_{3}+e_{4}+\sum_{j \equiv 0}\left(e_{1,1}^{j}+e_{2,2}^{j}\right)\right)$;

- $J_{m}=I\left(e_{1}+e_{4}+\sum_{j \equiv 0} p_{1,1}^{j}+\sum_{j \equiv 2} p_{2,2}^{j}\right)$.

$K_{0}$ is a Kac subalgebra of $K_{4}$ and $J_{20}$ is contained in $K_{1}$.

For $n=4 m^{\prime}+2$ : The Jones projection of $K_{0}$ is $e_{1}+\sum_{j=1}^{m^{\prime}} p_{1,1}^{4 j}$. The other central projection in the same connected component of the Bratelli diagram of $K_{0} \subset$ $K D(n)$ is $e_{2}+\sum_{j \equiv 0} p_{2,2}^{j}$. The coideal subalgebras not contained in $J_{0}$ are:

- $I_{3}=I\left(e_{1}+e_{2}+\sum_{j \equiv 0}\left(e_{1,1}^{j}+e_{2,2}^{j}\right)+\sum_{j \equiv 1} r_{2,2}^{j}+\sum_{j \equiv 3} r_{1,1}^{j}\right)$;

- $I_{4}=I\left(e_{1}+e_{2}+\sum_{j \equiv 0}\left(e_{1,1}^{j}+e_{2,2}^{j}\right)+\sum_{j \equiv 1} r_{1,1}^{j}+\sum_{j \equiv 3} r_{2,2}^{j}\right)$;

- $J_{20}=I\left(e_{1}+e_{2}+\sum_{j \equiv 0}\left(e_{1,1}^{j}+e_{2,2}^{j}\right)\right)$;

- $J_{m}=I\left(e_{1}+e_{3}+\sum_{j \equiv 0} p_{1,1}^{j}+\sum_{j \equiv 2} p_{2,2}^{j}\right)$.

From 2.4.2 (5), $J_{m}$ is contained in $K_{3}$.

In both cases, $J_{20}$ is the coideal subalgebra of functions which are constant on the right cosets for $\left\langle\alpha^{2}\right\rangle$ in $K_{2}$ (see 5.7). Recall that, by [2.8.6, all the coideal subalgebras of dimension 2 of $K D(n)$ are in $K_{0}$.

7.1.3. Principal graphs coming from $K_{0}$ and its coideal subalgebras. Since $K D(n)$ is not self-dual when $n$ is even, the Bratelli diagram of an inclusion $I \subset K D(n)$ yields by 2.5 the principal graph of an intermediate factor in $\widehat{K D(n)}$. With the notations of 4 , here are the principal graphs coming from the coideal subalgebras of $K_{0}$ :

- The inclusions $N_{1} \subset N_{1} \rtimes \delta\left(I_{0}\right)$ and $R \subset R \rtimes \delta\left(J_{20}\right)$ are of depth 2;

- The principal graph of $N_{1} \subset N_{1} \rtimes \delta\left(I_{1}\right)$ and of $N_{1} \subset N_{1} \rtimes \delta\left(I_{2}\right)$ is $D_{2 n} / \mathbb{Z}_{2}$;

- The principal graph of $N_{1} \subset N_{1} \rtimes \delta\left(I_{3}\right)$ and of $N_{1} \subset N_{1} \rtimes \delta\left(I_{4}\right)$ is $Q B_{m^{\prime}}$ for $n=4 m^{\prime}$ and $D B_{m^{\prime}}$ for $n=4 m^{\prime}+2$;

- The principal graph of $N_{1} \subset N_{1} \rtimes \delta\left(J_{0}\right)$ and of $N_{1} \subset N_{1} \rtimes \delta\left(J_{m}\right)$ is $D_{n} / \mathbb{Z}_{2}$ (see 7.5);

- The principal graph of $N_{1} \subset N_{1} \rtimes \delta\left(K_{0}\right)$ is $D_{m} / \mathbb{Z}_{2}$.

7.2. The Kac subalgebra $K_{4}=I\left(e_{1}+e_{4}\right)$. As in 5.4.2, consider the Kac subalgebra of $K D(n)$ isomorphic to $K D(m)$ and generated by $\lambda\left(a^{2}\right)$ and $\lambda(b)$. Its dimension is $2 n$, and it contains $e_{1}+e_{4}$ (see A.2). Therefore, it coincides with $K_{4}$.

From the formulas of A.2 or A.5.2,

$$
K_{4}=\mathbb{C}\left(e_{1}+e_{4}\right) \oplus \mathbb{C}\left(e_{2}+e_{3}\right) \oplus \mathbb{C} p_{1,1}^{m} \oplus \mathbb{C} p_{2,2}^{m} \oplus \bigoplus_{j=1}^{m-1} K_{4}^{j},
$$

where $K_{4}^{j}$ is the factor $M_{2}(\mathbb{C})$ with matrix units $e_{1,1}^{j}+e_{2,2}^{n-j}, e_{1,2}^{j}+e_{2,1}^{n-j}, e_{2,1}^{j}+e_{1,2}^{n-j}$, and $e_{2,2}^{j}+e_{1,1}^{n-j}$ (note: $j$ and $n-j$ have the same parity).

7.3. The Kac subalgebra $K_{3}=I\left(e_{1}+e_{3}\right)$. Since $\Delta\left(e_{1}+e_{3}\right)$ is symmetric (see A.5.2), by 2.8.3, $K_{3}$ is a Kac subalgebra of $K D(n)$.

From the expression of the coproduct of the Jones projection, we deduce: if $m$ is odd:

$$
K_{3}=I\left(e_{1}+e_{3}\right)=\mathbb{C}\left(e_{1}+e_{3}\right) \oplus \mathbb{C}\left(e_{2}+e_{4}\right) \oplus \mathbb{C} e_{1,1}^{m} \oplus \mathbb{C} e_{2,2}^{m} \oplus \bigoplus_{j=1}^{m-1} K_{3}^{j},
$$

if $m$ is even:

$$
K_{3}=I\left(e_{1}+e_{3}\right)=\mathbb{C}\left(e_{1}+e_{3}\right) \oplus \mathbb{C}\left(e_{2}+e_{4}\right) \oplus \mathbb{C} r_{1,1}^{m} \oplus \mathbb{C} r_{2,2}^{m} \oplus \bigoplus_{j=1}^{m-1} K_{3}^{j},
$$


where in both cases $K_{3}^{j}$ is the factor $M_{2}(\mathbb{C})$ with matrix units:

for $j$ even:

for $j$ odd:

$$
e_{2,2}^{j}+e_{1,1}^{n-j}, \quad e_{2,1}^{j}-e_{1,2}^{n-j}, e_{1,2}^{j}-e_{2,1}^{n-j}, \quad \text { and } \quad e_{1,1}^{j}+e_{2,2}^{n-j}
$$

$$
\begin{aligned}
& r_{2,2}^{j}+r_{1,1}^{n-j}, \quad r_{2,1}^{j}-r_{1,2}^{n-j}, \quad r_{1,2}^{j}-r_{2,1}^{n-j}, \quad \text { and } \quad r_{1,1}^{j}+r_{2,2}^{n-j} \text {, } \\
& \text { or } \\
& e_{1,1}^{j}+e_{1,1}^{n-j}, \quad e_{2,1}^{j}-e_{2,1}^{n-j}, \quad e_{1,2}^{j}-e_{1,2}^{n-j}, \quad \text { and } \quad e_{2,2}^{j}+e_{2,2}^{n-j} .
\end{aligned}
$$

$K_{3}$ is further studied in Section 9, in connexion with the families of Kac algebras $K Q(m)$ and $K B(m)$.

7.4. The Kac subalgebra $K_{1}=I\left(e_{1}+e_{2}+e_{3}+e_{4}\right)$.

Proposition 7.4. For $n=2 m, K_{1}=I\left(e_{1}+e_{2}+e_{3}+e_{4}\right)=K_{2} \cap K_{3} \cap K_{4}$ is a commutative Kac subalgebra isomorphic to $L^{\infty}\left(D_{m}\right)$. Its matrix structure is given by

$$
K_{1}=\mathbb{C}\left(e_{1}+e_{2}+e_{3}+e_{4}\right) \oplus \bigoplus_{j=1, j \text { odd }}^{n-1} \mathbb{C}\left(r_{1,1}^{j}+r_{2,2}^{n-j}\right) \oplus \bigoplus_{j=1, j \text { even }}^{n-1} \mathbb{C}\left(e_{1,1}^{j}+e_{2,2}^{n-j}\right)
$$

Its lattice of coideal subalgebras is the dual of the lattice of subgroups of $D_{m}$.

In $K_{2} \equiv L^{\infty}\left(D_{2 m}\right)$ it is the subalgebra of constant functions on the right cosets of $\left\{1, \alpha^{m}\right\}$. In $K_{4} \equiv K D(m), K_{1}$ plays the role of $K_{2}$.

Proof. The commutative subalgebra $K_{1}$ has to play the role of $K_{2}$ in $K_{4}$ because $K_{4} \equiv$ $K D(m)$ has a single commutative coideal subalgebra of this dimension. The rest follows from 5.7, 5.9, and A.5.2.

7.5. The coideal subalgebras of dimension 4. Recall that, from 5.10, any coideal subalgebra of dimension 4 is contained in some $K_{i}$. In this section, we use this fact to inductively describe all the coideal subalgebras of dimension 4 of $K D(2 m)$. As in the case $n$ odd (see A.4), we exhibit $2 m$ projections that generate $2 m$ coideal subalgebras $J_{k}$ of dimension 4:

Lemma 7.5. For $k=0, \ldots, n-1$, define

$$
p_{J_{k}}=e_{1}+e_{i}+\sum_{j=1}^{m-1} q\left(0, \frac{2 k j \pi}{n}, 2 j\right),
$$

where $i=3$ (resp. $i=4$ ) for $k$ odd (resp. even). The projection $p_{J_{k}}$ is the Jones projection of a coideal subalgebra $J_{k}$ of dimension 4 of $K_{3}$ (resp. $K_{4}$ ) for $k$ odd (resp. even). In particular, $J_{0}$ and $J_{m}$ are contained in $K_{0}$.

Furthermore, the principal graph of the inclusion $N_{1} \subset N_{1} \rtimes \delta\left(J_{k}\right)$ is $D_{n} / \mathbb{Z}_{2}$ (see 4.2 ).

Proposition 7.5. The coideal subalgebras of dimension 4 of $K D(2 m)$ are:

- in $K_{3}$ : the $m$ coideal subalgebras $J_{k}, k$ odd;

- in $K_{4}$ : the $m$ coideal subalgebras $J_{k}, k$ even;

- in $K_{2}$ :

- when $m$ is odd, the unique coideal subalgebra $J_{20}$ of dimension 4;

- when $m$ is even, the five coideal subalgebras of dimension 4.

Proof. Case $m$ odd: $K_{3}$ has $m$ coideal subalgebras of dimension 4 since it is isomorphic to $K Q(m)$ (see 8.4); $K_{4}$ has $m$ coideal subalgebras of dimension 4 since it is isomorphic to $K D(m)$ (see 6.7). The unique coideal subalgebra of dimension 4 of $K_{2}$ is $J_{20}$; it corresponds to the unique subgroup of order $m$ of $D_{n}$ (see 5.7). 
Case $m=2 m^{\prime}$ even: $D_{n}$ has five subgroups of order $m$, and the associated coideal subalgebras of $K_{2}$ can be made explicit from the results of [5.8, which we will do in the examples. We prove in 9.3 that the coideal subalgebras of dimension 4 of $K_{3}$ are either in $K_{1}$, or are the $J_{k}$ for $k$ odd. Since $K_{4}$ is isomorphic to $K D\left(2 m^{\prime}\right)$, we can use induction: we shall see in 7.6 that the proposition holds for $m=2$. Assume that the proposition holds for $m^{\prime}$; then the dimension 4 coideal subalgebras of $K_{4}$ are:

- the coideal subalgebras $J_{k}$ of $K D\left(2 m^{\prime}\right)$ which give the $J_{2 k}$ in $K_{4}$;

- those of the $K_{2}$ of $K D\left(2 m^{\prime}\right)$ which itself is the subalgebra $K_{1}$ of $K_{2}$ of $K D(2 m)$.

So the proposition holds for $m$.

7.6. The Kac algebra $K D(4)$ of dimension 16. We now illustrate the general study of $K D(2 m)$ with $m$ even on the Kac algebra $K D(4)$ of dimension 16. This algebra (and its dual $\widehat{K D(4)}$ with underlying algebra $\left.\mathbb{C}^{8} \oplus M_{2}(\mathbb{C}) \oplus M_{2}(\mathbb{C})\right)$ are described in [K02, XI.15].

Proposition 7.6. The lattice of coideal subalgebras of $K D(4)$ is as given in Figure 12.

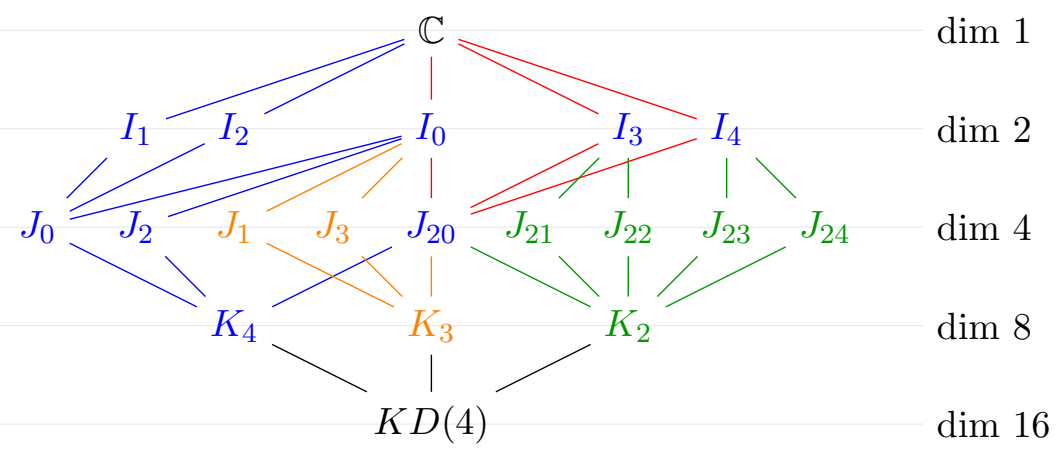

Figure 12. The lattice of coideal subalgebras of $K D(4)$

Proof. From 5.10, any coideal subalgebra of $K D(4)$ is inductively a coideal subalgebra of one of the three Kac subalgebras of dimension 8: $K_{2}, K_{3}$, and $K_{4}$. In the sequel of this section, we study them in turn.

The coproduct expressions which we use in this section are collected in A.5.2.

7.6.1. The coideal subalgebras of $K_{4}$. The Kac subalgebra $K_{4}=I\left(e_{1}+e_{4}\right)$ is isomorphic to $K D(2)$ :

$$
K_{4}=\mathbb{C}\left(e_{1}+e_{4}\right) \oplus \mathbb{C}\left(e_{2}+e_{3}\right) \oplus \mathbb{C} q_{1} \oplus \mathbb{C} q_{2} \oplus M_{2}(\mathbb{C}),
$$

where the matrix units of the factor $M_{2}(\mathbb{C})$ are:

$$
e_{1,1}^{1}+e_{2,2}^{3}, \quad e_{1,2}^{1}+e_{2,1}^{3}, \quad e_{2,1}^{1}+e_{1,2}^{3}, \quad \text { and } \quad e_{2,2}^{1}+e_{1,1}^{3} .
$$

As a special feature of $K D(4), K_{4}$ coincides with the group algebra $K_{0}$ of the intrinsic group studied in 7.1.2. The element $c=e_{1}-e_{2}-e_{3}+e_{4}-i\left(e_{1,1}^{1}-e_{2,2}^{1}\right)-\left(q_{1}-q_{2}\right)+i\left(e_{1,1}^{3}-e_{2,2}^{3}\right)$ of $K_{4}$ is group-like and of order 4 , and its square is $\lambda\left(a^{4}\right)$. The intrinsic group $D_{4}$ of $K D(4)$ is therefore generated by $c$ and $\lambda(b)$.

The coideal subalgebras of $K_{4}$ are the algebras of the subgroups of $D_{4}$ (see 7.1.2). In dimension 2, they are:

- $I_{0}=\mathbb{C}\left(e_{1}+e_{2}+e_{3}+e_{4}+q_{1}+q_{2}\right) \oplus \mathbb{C}\left(p_{1}+p_{2}\right)$ generated by $\lambda\left(a^{4}\right)$;

- $I_{1}=\mathbb{C}\left(e_{1}+e_{4}+q_{1}+p_{2}\right) \oplus \mathbb{C}\left(e_{2}+e_{3}+p_{1}+q_{2}\right)$ generated by $\lambda\left(b a^{4}\right)$;

- $I_{2}=\mathbb{C}\left(e_{1}+e_{4}+p_{1}+q_{1}\right) \oplus \mathbb{C}\left(e_{2}+e_{3}+p_{2}+q_{2}\right)$ generated by $\lambda(b)$; 
- $I_{3}=I\left(e_{1}+e_{2}+e_{3}+e_{4}+r_{2,2}^{1}+r_{1,1}^{3}\right)$ generated by $\lambda(b) c$;

- $I_{4}=I\left(e_{1}+e_{2}+e_{3}+e_{4}+r_{1,1}^{1}+r_{2,2}^{3}\right)$ generated by $\lambda(b) c^{3}$.

Since $K_{4}=K_{0}$, those give all the coideal subalgebras of dimension 2 of $K D(4)$.

In dimension 4 , the coideal subalgebras are:

- $J_{0}=\mathbb{C}\left(e_{1}+e_{4}+q_{1}\right) \oplus \mathbb{C}\left(e_{2}+e_{3}+q_{2}\right) \oplus \mathbb{C} p_{2} \oplus \mathbb{C} p_{1}$ generated by $\lambda\left(a^{4}\right)$ and $\lambda(b)$;

- $J_{20}=\mathbb{C}\left(e_{1}+e_{2}+e_{3}+e_{4}\right) \oplus \mathbb{C}\left(q_{1}+q_{2}\right) \oplus \mathbb{C}\left(r_{1,1}^{1}+r_{2,2}^{3}\right) \oplus \mathbb{C}\left(r_{2,2}^{1}+r_{1,1}^{3}\right)$ generated by $c^{2}$ and $\lambda(b) c$;

- $J_{2}=\mathbb{C}\left(e_{1}+e_{4}+q_{2}\right) \oplus \mathbb{C}\left(e_{2}+e_{3}+q_{1}\right) \oplus \mathbb{C}\left(e_{1,1}^{1}+e_{2,2}^{3}\right) \oplus \mathbb{C}\left(e_{2,2}^{1}+e_{1,1}^{3}\right)$ generated by $\lambda(c)$.

7.6.2. The coideal subalgebras of $K_{3}$. From 7.3 .

$$
K_{3}=I\left(e_{1}+e_{3}\right)=\mathbb{C}\left(e_{1}+e_{3}\right) \oplus \mathbb{C}\left(e_{2}+e_{4}\right) \oplus \mathbb{C} r_{1,1}^{2} \oplus \mathbb{C} r_{2,2}^{2} \oplus M_{2}(\mathbb{C}),
$$

where the matrix units of the factor $M_{2}(\mathbb{C})$ are

$$
e_{1,1}^{1}+e_{1,1}^{3}, \quad e_{1,2}^{1}-e_{1,2}^{3}, \quad e_{2,1}^{1}-e_{2,1}^{3}, \quad \text { and } \quad e_{2,2}^{1}+e_{2,2}^{3} .
$$

Since the coproduct of $r_{1,1}^{2}$ is not symmetric (checked on computer), $K_{3}$ is a non trivial Kac algebra of dimension 8 , and therefore isomorphic to $K P$. Its coideal subalgebras are $I_{0}, I_{3}$, and $I_{4}$ contained in $J_{20}$, and $J_{1}$ and $J_{3}$ which contains $I_{0}$ (see 3.3):

$$
\begin{aligned}
J_{1}=I\left(e_{1}+e_{3}+r_{1,1}^{2}\right) & =\mathbb{C}\left(e_{1}+e_{3}+r_{1,1}^{2}\right) \oplus \mathbb{C}\left(e_{2}+e_{4}+r_{2,2}^{2}\right) \\
& \oplus \mathbb{C}(q(\pi / 4,0,1)+q(\pi / 4, \pi, 3)) \oplus \mathbb{C}(q(-\pi / 4, \pi, 1)+q(-\pi / 4,0,3)), \\
J_{3}=I\left(e_{1}+e_{3}+r_{2,2}^{2}\right) & =\mathbb{C}\left(e_{1}+e_{3}+r_{2,2}^{2}\right) \oplus \mathbb{C}\left(e_{2}+e_{4}+r_{1,1}^{2}\right) \\
& \oplus \mathbb{C}(q(-\pi / 4,0,1)+q(-\pi / 4, \pi, 3)) \oplus \mathbb{C}(q(\pi / 4, \pi, 1)+q(\pi / 4,0,3)) .
\end{aligned}
$$

The principal graph of $N_{1} \subset N_{1} \rtimes \delta\left(J_{i}\right), i=1,3$ is $D_{6}^{(1)}$.

7.6.3. The coideal subalgebras of $K_{2}$. From 5.7, the commutative Kac subalgebra $K_{2}$ is the algebra $L^{\infty}\left(D_{4}\right)$ of functions on the group $D_{4}$, its basis is given by:

$K_{2}=I\left(e_{1}+e_{2}\right)=\mathbb{C}\left(e_{1}+e_{2}\right) \oplus \mathbb{C}\left(e_{3}+e_{4}\right) \oplus \mathbb{C} r_{1,1}^{1} \oplus \mathbb{C} r_{2,2}^{1} \oplus \mathbb{C} e_{1,1}^{2} \oplus \mathbb{C} e_{2,2}^{2} \oplus \mathbb{C} r_{1,1}^{3} \oplus \mathbb{C} r_{2,2}^{3}$ and its lattice of coideal subalgebras is in correspondence with the dual of the lattice of the subgroups of $D_{4}$. There are three coideal subalgebras of dimension 2 contained in $J_{20}$ and four coideal subalgebras of dimension 4 :

$$
\begin{aligned}
& J_{21}=\mathbb{C}\left(e_{1}+e_{2}+r_{1,1}^{1}\right) \oplus \mathbb{C}\left(e_{3}+e_{4}+r_{2,2}^{3}\right) \oplus \mathbb{C}\left(e_{2,2}^{2}+r_{1,1}^{3}\right) \oplus \mathbb{C}\left(r_{2,2}^{1}+e_{1,1}^{2}\right) ; \\
& J_{22}=\mathbb{C}\left(e_{1}+e_{2}+r_{2,2}^{1}\right) \oplus \mathbb{C}\left(e_{3}+e_{4}+r_{1,1}^{3}\right) \oplus \mathbb{C}\left(e_{1,1}^{2}+r_{2,2}^{3}\right) \oplus \mathbb{C}\left(r_{1,1}^{1}+e_{2,2}^{2}\right) ; \\
& J_{23}=\mathbb{C}\left(e_{1}+e_{2}+r_{2,2}^{3}\right) \oplus \mathbb{C}\left(e_{3}+e_{4}+r_{1,1}^{1}\right) \oplus \mathbb{C}\left(e_{1,1}^{2}+r_{1,1}^{3}\right) \oplus \mathbb{C}\left(r_{1,1}^{1}+e_{2,2}^{2}\right) ; \\
& J_{24}=\mathbb{C}\left(e_{1}+e_{2}+r_{1,1}^{3}\right) \oplus \mathbb{C}\left(e_{3}+e_{4}+r_{2,2}^{1}\right) \oplus \mathbb{C}\left(e_{2,2}^{2}+r_{2,2}^{3}\right) \oplus \mathbb{C}\left(r_{1,1}^{1}+e_{1,1}^{2}\right) .
\end{aligned}
$$

For $j=21, \ldots, 24$, the principal graph of $N_{1} \subset N_{1} \rtimes \delta\left(J_{j}\right)$ is $D_{10}^{(1)}$.

7.7. The Kac algebra $K D(6)$ of dimension 24. The study of the lattice of the coideal subalgebras of $K D(6)$ illustrates how a large part of it (but not all!) can be constructed inductively.

Theorem 7.7. The lattice of coideal subalgebras of $K D(6)$ is as given in Figure 13 .

Proof. From 5.10 and 9.2, any coideal subalgebras not of dimension 8 is contained in one of the three Kac subalgebras of dimension $12: K_{2} \equiv L^{\infty}\left(D_{n}\right), K_{3} \equiv K Q(3)$, and $K_{4} \equiv K D(3)$ which we study in turn in the sequel of this section.

In 7.7.6 we construct all coideal subalgebras of dimension 8 . 


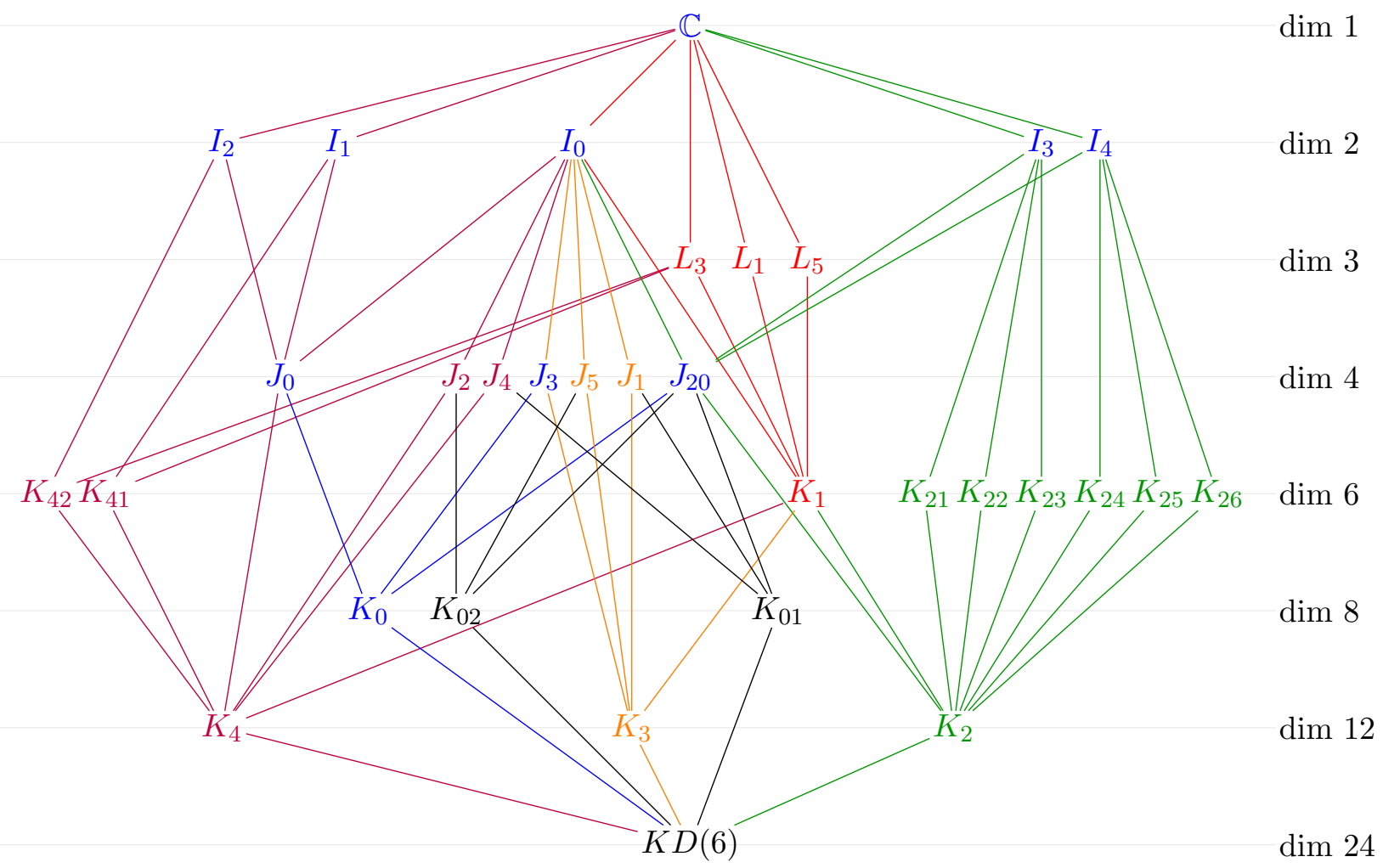

Figure 13. The lattice of coideal subalgebras of $K D(6)$

7.7.1. The coideal subalgebras of $K_{0}$. From 7.1, $K_{0}=I\left(e_{1}+p_{1,1}^{4}\right)$ is the algebra of the intrinsic group $D_{4}$ and is of dimension 8. It contains all the coideal subalgebras of dimension 2 of $K D(6)$. It also contains the algebra $J_{0}$ of the subgroup $H$. Its coideal subalgebras are:

- $I_{0}=\mathbb{C}\left(e_{1}+e_{2}+e_{3}+e_{4}+q_{1}+q_{2}\right)$

- $I_{1}=\mathbb{C}\left(e_{1}+e_{4}+q_{1}+p_{2}\right)$

- $I_{2}=\mathbb{C}\left(e_{1}+e_{4}+q_{1}+p_{1}\right)$

- $I_{3}=I\left(e_{1}+e_{2}+e_{1,1}^{4}+e_{2,2}^{4}+r_{2,2}^{1}+r_{1,1}^{3}+r_{2,2}^{5}\right)$

- $I_{4}=I\left(e_{1}+e_{2}+e_{1,1}^{4}+e_{2,2}^{4}+r_{1,1}^{1}+r_{2,2}^{3}+r_{1,1}^{5}\right)$

- $J_{0}=\mathbb{C}\left(e_{1}+e_{4}+q_{1}\right)$

- $J_{20}=I\left(e_{1}+e_{2}+e_{1,1}^{4}+e_{2,2}^{4}\right)$

- $J_{3}=I\left(e_{1}+e_{3}+p_{1,1}^{4}+p_{2,2}^{2}\right)$

7.7.2. The coideal subalgebras of $K_{2}$. From 5.7, the Kac subalgebra $K_{2}=I\left(e_{1}+e_{2}\right)$ is $L^{\infty}\left(D_{6}\right)$. Its coideal subalgebras of dimension 6 correspond to the seven subgroups of order 2 of $D_{6}$ :

- $K_{1}=I\left(e_{1}+e_{2}+e_{3}+e_{4}\right)$ which is isomorphic to $L^{\infty}\left(D_{3}\right)$;

- $K_{21}=I\left(e_{1}+e_{2}+r_{1,1}^{1}\right)$;

- $K_{22}=I\left(e_{1}+e_{2}+r_{1,1}^{3}\right)$

- $K_{23}=I\left(e_{1}+e_{2}+r_{1,1}^{5}\right)$

- $K_{24}=I\left(e_{1}+e_{2}+r_{2,2}^{1}\right)$;

- $K_{25}=I\left(e_{1}+e_{2}+r_{2,2}^{3}\right)$;

- $K_{26}=I\left(e_{1}+e_{2}+r_{2,2}^{5}\right)$. 
The group $D_{6}=\langle\alpha, \beta\rangle$ has a single subgroup of order 3 which gives the coideal subalgebra $J_{1}$ of dimension 4 , and three subgroups of order 4 which give three coideal subalgebras of dimension 3:

- $L_{1}=I\left(e_{1}+e_{2}+e_{3}+e_{4}+r_{1,1}^{1}+r_{2,2}^{5}\right)$;

- $L_{3}=I\left(e_{1}+e_{2}+e_{3}+e_{4}+r_{1,1}^{3}+r_{2,2}^{3}\right)$;

- $L_{5}=I\left(e_{1}+e_{2}+e_{3}+e_{4}+r_{1,1}^{5}+r_{2,2}^{1}\right)$.

Finally, the coideal subalgebras of dimension 2 of $K_{2}$ are $I_{0}, I_{3}$ et $I_{4}$ (see 2.4.2 (5)).

7.7.3. The coideal subalgebras of $K_{3}$. The Kac algebra $K_{3}=I\left(e_{1}+e_{3}\right)$ is isomorphic to $K Q(3)$, and we can derive its coideal subalgebras from 8.4.3. There is a single coideal subalgebra of dimension 6: $K_{1}$. Its coideal subalgebras of dimension 2 and 3 are those of $K_{1}$ which we treated above. The three coideal subalgebras of dimension 4 are:

- $J_{3}$, which is the algebra of its intrinsic group,

- $J_{5}=I\left(e_{1}+e_{3}+q\left(0, \frac{5 \pi}{3}, 2\right)+q\left(0, \frac{4 \pi}{3}, 4\right)\right)$,

- $J_{1}=I\left(e_{1}+e_{3}+q\left(0, \frac{\pi}{3}, 2\right)+q\left(0, \frac{2 \pi}{3}, 4\right)\right)$.

We computed those projections by using successively the embedding of $K Q(3)$ into $K Q(6)$ and the explicit isomorphism from $K Q(6)$ to $K D(6)$ (see B.5.4).

7.7.4. The coideal subalgebras of $K_{4}$. The Kac subalgebra $K_{4}=I\left(e_{1}+e_{4}\right)$ is isomorphic to $K D(3)$, and we can derive its coideal subalgebras from 6.9.1, Besides $K_{1}$, it contains two other coideal subalgebras of dimension 6 :

- $K_{41}=I\left(e_{1}+e_{4}+p(2,2,3)\right)$ which contains $I_{1}$ and $L_{3}$,

- $K_{42}=I\left(e_{1}+e_{4}+p(1,1,3)\right)$ which contains $I_{2}$ and $L_{3}$.

The coideal subalgebras of dimension 4 are:

- $J_{0}$, which is the algebra of its intrinsic group,

- $J_{2}=I\left(e_{1}+e_{4}+q\left(0, \frac{2 \pi}{3}, 2\right)+q\left(0, \frac{4 \pi}{3}, 4\right)\right)$,

- $J_{4}=I\left(e_{1}+e_{4}+q\left(0, \frac{4 \pi}{3}, 2\right)+q\left(0, \frac{2 \pi}{3}, 4\right)\right)$.

We computed those projections by using the embedding of $K D(3)$ in $K D(6)$ (see B.5.4).

7.7.5. The coideal subalgebras of dimension 4 of $K D(6)$. As predicted by Proposition 7.5, there are seven coideal subalgebras of dimension 4 in $K D(6): J_{20}$ and $J_{k}$, with $k=0 \ldots 5$.

7.7.6. The coideal subalgebras of dimension 8 of $K D(6)$.

Proposition 7.7.6. The coideal subalgebras of dimension 8 of $K D(6)$ are the following:

- $K_{0}=I\left(e_{1}+p_{1,1}^{4}\right)$, the algebra of the intrinsic group;

- $K_{01}=I\left(e_{1}+q\left(0, \frac{2 \pi}{3}, 4\right)\right)=I\left(p_{\left\langle a^{3}, b a\right\rangle}\right)$, which contains $J_{20}, J_{1}$, and $J_{4}$;

- $K_{02}=I\left(e_{1}+q\left(0, \frac{4 \pi}{3}, 4\right)\right)=I\left(p_{\left\langle a^{3}, a b\right\rangle}\right)$, which contains $J_{20}, J_{2}$, and $J_{5}$.

The last two are isomorphic by $\Theta$; they share the same block matrix structure as KP, and their lattice of coideal subalgebras are isomorphic to that of KP. However they are not Kac subalgebras.

Proof. We first prove that any coideal subalgebra of dimension 8 in $K D(6)$ contains $J_{20}$ and that its Jones projection is of the form $e_{1}+s$, with $s$ a minimal projection of the fourth factor $M_{2}(\mathbb{C})$ of $K D(6)$ :

By a usual trace argument, the Jones projection of a coideal subalgebra $K$ of dimension 8 is of the form $e_{1}+s$, with $s$ a minimal projection of some $j$-th factor $M_{2}(\mathbb{C}$ ) (in principle, it could also be of the form $e_{1}+e_{i}+e_{k}$, but we ruled out those later projections by a systematic check on computer). The index of $N_{1} \subset N_{1} \rtimes \delta(K)$ is 3. Given the shape of $p_{K}$, its principal graph is $A_{5}$. Therefore, the center of $K$ admits a projection of the form $e_{i}+\left(e_{1,1}^{j}+e_{2,2}^{j}-s\right)$ (with $i=2,3$, or 4 ). Then, the coideal subalgebra generated 
by $e_{1}+e_{i}+e_{1,1}^{j}+e_{2,2}^{j}$ is of dimension at most 8 since it is contained in $K$. A computer check on the 15 possible values shows that only $e_{1}+e_{2}+e_{1,1}^{4}+e_{2,2}^{4}$ satisfies this condition. Therefore, every coideal subalgebra of dimension 8 contains $J_{20}=I\left(e_{1}+e_{2}+e_{1,1}^{4}+e_{2,2}^{4}\right)$ and its Jones projection is of the form $e_{1}+s$, with $s$ a minimal projection of the fourth factor $M_{2}(\mathbb{C})$ of $K D(6)$.

Using Proposition 2.8.2 (4), we checked on computer that the only such Jones projections are $e_{1}+p_{1,1}^{4}, e_{1}+q\left(0, \frac{2 \pi}{3}, 4\right)$ and $e_{1}+q\left(0, \frac{4 \pi}{3}, 4\right)$; this reduced to solving a system of quadratic algebraic equations. We also checked that both new coideal subalgebras have the same block matrix structure as $K P$ but are not Kac subalgebras.

The mentioned inclusions are straightforward to check using Proposition 2.4.2 (5). 


\section{The KaC Algebras $K Q(n)$}

The structure of $K Q(n)$ follows closely that of $K D(n)$, and we sketch some general results. We first prove that $K Q(2 m)$ is isomorphic to $K D(2 m)$. Then, when $n$ is odd, we establish the self-duality of $K Q(n)$, describe its automorphism group, list its coideal subalgebras of dimension $4, n$ and $2 n$, and get a partial description of $1(K Q(n))$ (Theorem 8.4); this description is complete for $n$ odd prime. Finally, we complete the systematic study of all Kac algebras of dimension at most 15 by the lattice $1(K Q(3))$ which serves as intermediate step toward the lattice of $K D(6)$ in 7.7.

\subsection{Definition and general properties.}

8.1.1. Notations. For $n \geq 1$, we denote by $K Q(n)$ the Kac algebra of dimension $4 n$ constructed in [Vai98, 6.6] from the quaternion group $Q_{n}=\left\langle a, b \mid a^{2 n}=1, b^{2}=a^{n}, b a=a^{-1} b\right\rangle$. As for $K D(n)$, its block matrix structure is:

$$
K Q(n)=\mathbb{C} e_{1}^{\prime} \oplus \mathbb{C} e_{2}^{\prime} \oplus \mathbb{C} e_{3}^{\prime} \oplus \mathbb{C} e_{4}^{\prime} \oplus \bigoplus_{k=1}^{n-1} K Q(n)^{k},
$$

where $K Q(n)^{k}$ is a factor $M_{2}(\mathbb{C})$ whose matrix units we denote by $e_{i, j}^{\prime k}, i=1,2, j=1,2$. In general, we use the same notations for matrix units as in 5.2, and put a ' when needed to distinguish between the matrix units of $K D(n)$ and $K Q(n)$.

From 2.8.1, the trace on $K Q(n)$ is given by: $\operatorname{tr}\left(e_{i}^{\prime}\right)=1 / 4 n \operatorname{tr}\left(e_{i, j}^{\prime k}\right)=1 / 2 n$.

Setting $\epsilon_{n}=\mathrm{e}^{\mathrm{i} \pi / n}$, the left regular representation of $Q_{n}$ is given by:

$$
\begin{aligned}
& \lambda^{\prime}\left(a^{k}\right)=e_{1}^{\prime}+e_{2}^{\prime}+(-1)^{k}\left(e_{3}^{\prime}+e_{4}^{\prime}\right)+\sum_{j=1}^{n-1}\left(\epsilon_{n}^{j k} e_{1,1}^{\prime j}+\epsilon_{n}^{-j k} e_{2,2}^{\prime j}\right) ; \\
& \lambda^{\prime}\left(a^{k} b\right)=e_{1}^{\prime}-e_{2}^{\prime}+(-1)^{k}\left(e_{3}^{\prime}-e_{4}^{\prime}\right)+\sum_{j=1}^{n-1}\left(\epsilon_{n}^{j(k-n)} e_{1,2}^{\prime j}+\epsilon_{n}^{-j k} e_{2,1}^{\prime j}\right) \quad \text { for } n \text { even } ; \\
& \lambda^{\prime}\left(a^{k} b\right)=e_{1}^{\prime}-e_{2}^{\prime}+(-1)^{k} \mathrm{i}\left(e_{3}^{\prime}-e_{4}^{\prime}\right)+\sum_{j=1}^{n-1}\left(\epsilon_{n}^{j(k-n)} e_{1,2}^{\prime j}+\epsilon_{n}^{-j k} e_{2,1}^{\prime j}\right) \quad \text { for } n \text { odd } .
\end{aligned}
$$

The coproduct of $\mathbb{C}\left[Q_{n}\right]$ is twisted by a 2-pseudo-cocycle $\Omega^{\prime}$, given in 8.4.1, We refer to [Vai98, 6.6] for details. As in 5.3 one can show that $K Q(1)$ and $K Q(2)$ are respectively the algebras of the groups $\mathbb{Z}_{4}$ and $D_{4}$.

8.1.2. Intrinsic group. In [Vai98, p.718], L. Vainerman mentions that the intrinsic group of the dual of $K Q(n)$ is $\mathbb{Z}_{4}$ if $n$ is odd, and $\mathbb{Z}_{2} \times \mathbb{Z}_{2}$ otherwise. As in 5.4.3, one can show that the intrinsic group of $K Q(n)$ is $\mathbb{Z}_{4}$ if $n$ is odd and $D_{4}$ otherwise.

\subsubsection{Automorphism group.}

Theorem 8.1.3. For $n \geq 3$, the automorphism group $\operatorname{Aut}(K Q(n))$ of $K Q(n)$ is given by:

$$
A_{2 n}=\left\{\Theta_{k}, \Theta_{k} \Theta^{\prime} \mid k \wedge 2 n=1\right\},
$$

where $\Theta_{k}$ and $\Theta^{\prime}$ are defined as in Theorem 5.5.7. In particular, it is of order $2 \varphi(2 n)$ (where $\varphi$ is the Euler function), and isomorphic to $\mathbb{Z}_{2 n}^{*} \rtimes \mathbb{Z}_{2}$, where $\mathbb{Z}_{2 n}^{*}$ is the multiplicative group of $\mathbb{Z}_{2 n}$.

Proof. The proof follows that of Theorem 5.5.7. The analogue of Lemma 5.5.5 holds because $\Theta^{\prime}(a)$ lies in the subalgebra generated by $a$ in $K Q(n)$ which is isomorphic to that in $K D(n)$. Furthermore, the group automorphisms which fix $a^{n}$ and $b$ are the same in $D_{2 n}$ and $Q_{2 n}$. Everything else is checked on computer. 


\subsection{The isomorphism $\phi$ from $K D(2 m)$ to $K Q(2 m)$.}

Theorem 8.2. The Kac algebras $K D(n)$ and $K Q(n)$ are isomorphic if and only if $n$ is even. More specifically, when $n=2 m$, $\phi$ defined by:

$$
\begin{aligned}
& \phi(\lambda(a))=a+1 / 4\left(a-a^{-1}\right)\left(a^{n}-1\right)\left(1-b a^{m}\right), \\
& \phi(\lambda(b))=1 / 2\left[b\left(a^{n}+1\right)+\mathrm{i}\left(a^{n}-1\right)\right] a^{m},
\end{aligned}
$$

where for the sake of readability the $\lambda^{\prime}$ have been omitted on the right hand sides, extends into a Kac algebra isomorphism from $K D(n)$ to $K Q(n)$.

Note first that $K D(n)$ and $K Q(n)$ are not isomorphic for $n$ odd, as their intrinsic groups are $\mathbb{Z}_{2}^{2}$ and $\mathbb{Z}_{4}$, respectively (see 5.4.3 and 8.1.2). The proof of the isomorphism for $n$ even follows the same line as that of Proposition 5.5.2. We start with some little remarks which simplify the calculation, give an explicit formula for $\phi(\lambda(a))^{k}$, and conclude the proof with computer checks.

\subsubsection{Technical lemmas.}

Remarks 8.2.1. Recall that, in $Q_{n}, b a^{k} b^{-1}=a^{-k}, b^{4}=1$, and $b^{2}=a^{n}$. Therefore:

(i) $a^{n}$ is in the center of $K Q(n)$;

(ii) $\left(a^{n}-1\right)\left(a^{n}+1\right)=0$;

(iii) $\left(1+a^{n}\right)^{2}=2\left(1+a^{n}\right)$ et $\left(1-a^{n}\right)^{2}=2\left(1-a^{n}\right)$;

(iv) $\left(a^{k}-a^{-k}\right) b a^{m}=-b a^{m}\left(a^{k}-a^{-k}\right)$;

(v) $\left(1+b a^{m}\right)\left(1-b a^{m}\right)=1-a^{n}$.

Lemma 8.2.1. For all $k \in \mathbb{Z}$,

$$
\phi(\lambda(a))^{k}=\lambda^{\prime}\left(a^{k}\right)+\frac{1}{4}\left(\lambda^{\prime}\left(a^{k}\right)-\lambda^{\prime}\left(a^{-k}\right)\right)\left(\lambda^{\prime}\left(a^{n}\right)-1\right)\left(1-\lambda^{\prime}\left(b a^{m}\right)\right) .
$$

Furthermore $\phi\left(\lambda(a)^{*}\right)=\phi(\lambda(a))^{*}$.

Proof. Write $f_{k}=\lambda^{\prime}\left(a^{k}\right)+\frac{1}{4}\left(\lambda^{\prime}\left(a^{k}\right)-\lambda^{\prime}\left(a^{-k}\right)\right)\left(\lambda^{\prime}\left(a^{n}\right)-1\right)\left(1-\lambda^{\prime}\left(b a^{m}\right)\right)$. First note that $f_{0}=1$. One checks further that $f_{k} f_{1}=f_{k+1}$ (omitting $\left.\lambda^{\prime}\right)$ :

$$
\begin{aligned}
f_{k} f_{1}-a^{k+1} & =\frac{a^{n}-1}{4}\left[\left(1+b a^{m}\right)\left(a^{k}-a^{-k}\right) a+a^{k}\left(1+b a^{m}\right)\left(a-a^{-1}\right)\right] \\
& +\frac{\left(a^{n}-1\right)^{2}}{16}\left(1+b a^{m}\right)\left(a^{k}-a^{-k}\right)\left(a-a^{-1}\right)\left(1-b a^{m}\right) \\
& =\frac{a^{n}-1}{4}\left[\left(2 a^{k+1}-a^{-k+1}-a^{k-1}\right)+b a^{m}\left(a^{k+1}-a^{-k-1}\right)\right] \\
& \left.-\frac{\left(a^{n}-1\right)}{8}\left(1+b a^{m}\right)\left(1-b a^{m}\right)\left(a^{k+1}+a^{-k-1}-a^{k-1}-a^{-k+1}\right)\right] \\
& =\frac{a^{n}-1}{4}\left(1+b a^{m}\right)\left(a^{k+1}-a^{-k-1}\right) .
\end{aligned}
$$

In particular, we have $f_{-1}=f_{1}^{*}=f_{1}^{-1}$, that is $\phi\left(\lambda(a)^{*}\right)=\phi(\lambda(a))^{*}$. The lemma follows by induction.

\subsubsection{Proof of Theorem 8.2.}

Proof of Theorem 8.2. We check that $\phi$ satisfies the properties listed in Proposition 5.5.3, Most expressions below involve the element $a^{m}$. Therefore the computer checks use a close analogue of Proposition 5.5.4 for the group algebra $\mathbb{C}\left[Q_{2 \infty}\right]$ of

$$
Q_{2 \infty}=\left\langle a, b, a_{\infty} \mid b^{2}=a_{\infty}, a b=b a^{-1}, a_{\infty} b=b a_{\infty}^{-1}\right\rangle,
$$

where $a_{\infty}$ plays the role of $a^{m}$. Then, an algebraic expression of degree $d$ in $a$ vanishes in $K Q(2 m)$ for all $m$ whenever it vanishes for some $M>2 d$. 
(i) By Lemma 8.2.1, $\phi(\lambda(a))^{2 n}=1$, and we check on computer that $\phi(\lambda(b))^{2}=1$ and $\phi(\lambda(b)) \phi(\lambda(a))=\phi(\lambda(a))^{-1} \phi(\lambda(b))$.

(ii) By Lemma 8.2.1, $\phi\left(\lambda(a)^{*}\right)=\phi(\lambda(a))^{*}$, and check on computer that $\phi(\lambda(b))^{*} \phi(\lambda(b))=$ 1.

(iii) Using a close analogue of Proposition 5.5.4, we check on computer that $(\phi \otimes \phi)(\Delta(\lambda(x)))=$ $\Delta(\phi(x))$ for $x \in\{a, b\}$.

(iv) Similarly, we check on computer the following equations:

$$
\begin{aligned}
& \lambda^{\prime}(a)=\phi\left(\frac{1}{4}\left(\lambda(a)-\lambda(a)^{-1}\right)\left(\lambda(a)^{m}-\lambda(a)^{-m}\right)\left(\lambda(a)^{m}-\mathrm{i} \lambda(b)\right)+\lambda(a)\right), \\
& \lambda^{\prime}(b)=\phi\left(\frac{1}{2} \lambda(a)^{m}\left(\left(\lambda(a)^{n}+1\right) \lambda(b)-\left(\lambda(a)^{n}-1\right)\right)\right) .
\end{aligned}
$$

8.2.3. The isomorphism $\phi$ on the central projections. The following proposition gives the values of the isomorphism $\phi$ on the central projections of $K D(2 m)$.

Proposition 8.2.3. Set $n=2 m$. Then,

for $m$ even:

$$
\phi\left(e_{1}\right)=e_{1}^{\prime}, \quad \phi\left(e_{2}\right)=e_{2}^{\prime}, \quad \phi\left(e_{3}\right)=e_{4}^{\prime}, \quad \phi\left(e_{4}\right)=e_{3}^{\prime},
$$

and for $m$ odd:

$$
\phi\left(e_{1}\right)=e_{1}^{\prime}, \quad \phi\left(e_{2}\right)=e_{2}^{\prime}, \quad \phi\left(e_{3}\right)=e_{3}^{\prime}, \quad \phi\left(e_{4}\right)=e_{4}^{\prime} .
$$

Proof. We recall the following formulas (see A.2 and 8.1.1), where $\lambda$ (resp. $\lambda^{\prime}$ ) is the left regular representation of the dihedral (resp. quaternion) group:

$$
\begin{array}{rlrl}
e_{1}+e_{2} & =\frac{1}{2 n} \sum_{k=0}^{2 n-1} \lambda\left(a^{k}\right) & e_{1}^{\prime}+e_{2}^{\prime} & =\frac{1}{2 n} \sum_{k=0}^{2 n-1} \lambda^{\prime}\left(a^{k}\right) \\
e_{3}+e_{4} & =\frac{1}{2 n} \sum_{k=0}^{2 n-1}(-1)^{k} \lambda\left(a^{k}\right) & e_{3}^{\prime}+e_{4}^{\prime}=\frac{1}{2 n} \sum_{k=0}^{2 n-1}(-1)^{k} \lambda^{\prime}\left(a^{k}\right) \\
e_{1}-e_{2}=\lambda(b)\left(e_{1}+e_{2}\right) & e_{1}^{\prime}-e_{2}^{\prime}=\left(e_{1}^{\prime}+e_{2}^{\prime}\right) \lambda^{\prime}(b) \\
e_{3}-e_{4}=-\lambda(b)\left(e_{3}+e_{4}\right) & e_{3}^{\prime}-e_{4}^{\prime}=\left(e_{3}^{\prime}+e_{4}^{\prime}\right) \lambda^{\prime}(b)
\end{array}
$$

Using further the identities

$$
\sum_{k=0}^{2 n-1}\left(\lambda^{\prime}\left(a^{k}\right)-\lambda^{\prime}\left(a^{-k}\right)\right)=0 \quad \text { and } \quad \sum_{k=0}^{2 n-1}(-1)^{k}\left(\lambda^{\prime}\left(a^{k}\right)-\lambda^{\prime}\left(a^{-k}\right)\right)=0,
$$

it follows easily that $\phi\left(e_{1}+e_{2}\right)=e_{1}^{\prime}+e_{2}^{\prime}$ and $\phi\left(e_{3}+e_{4}\right)=e_{3}^{\prime}+e_{4}^{\prime}$. Then, from

$$
\lambda^{\prime}\left(a^{n}\right)=e_{1}^{\prime}+e_{2}^{\prime}+e_{3}^{\prime}+e_{4}^{\prime}+\sum_{j=1}^{n-1}(-1)^{j}\left(e_{1,1}^{j}+e_{2,2}^{j}\right)
$$

we get

$$
\left(e_{1}^{\prime}+e_{2}^{\prime}+e_{3}^{\prime}+e_{4}^{\prime}\right) \phi(\lambda(b))=\left(e_{1}^{\prime}-e_{2}^{\prime}+e_{3}^{\prime}-e_{4}^{\prime}\right) \lambda^{\prime}\left(a^{m}\right)=\left(e_{1}^{\prime}-e_{2}^{\prime}\right)+(-1)^{m}\left(e_{3}^{\prime}-e_{4}^{\prime}\right)
$$

and it follows that $\phi\left(e_{1}-e_{2}\right)=e_{1}^{\prime}-e_{2}^{\prime} \quad$ and $\quad \phi\left(e_{3}-e_{4}\right)=(-1)^{m+1}\left(e_{3}^{\prime}-e_{4}^{\prime}\right)$. 
8.3. Self-duality for $n$ odd. As for $K D(n), K Q(n)$ is self-dual for $n$ odd, and the proof follows the same lines (see 6.1).

Theorem 8.3. The Kac algebra $K Q(n)$ is self-dual if and only if $n$ is odd. When this is the case, one can take as Kac algebra isomorphism $\psi$ defined by:

$$
a \mapsto 2 n\left(\widehat{e_{1,1}^{n-1}}+\frac{1}{2}\left(i \widehat{e_{2,2}^{1}}-\widehat{e_{1,1}^{1}}\right)-\widehat{e_{1,2}^{n-1}}-\widehat{\left.e_{2,1}^{n-1}\right)}, \quad b \mapsto 4 n \widehat{e_{4}},\right.
$$

where the notations are as in 6.1.

The following proposition gives an alternative description of $\psi$ on the matrix units.

Proposition 8.3. Take $k$ odd in $\{1, \ldots, n-1$, and set:

$$
\begin{aligned}
E_{1,1}^{k} & =\chi_{b a^{n+k}}+\chi_{b a^{n-k}} \\
E_{2,2}^{k} & =\chi_{b a^{2 n-k}}+\chi_{b a^{k}} \\
E_{1,2}^{k} & =\frac{1}{2}\left[\left(-\chi_{a^{k}}+\chi_{a^{2 n-k}}+\chi_{a^{n+k}}-\chi_{a^{n-k}}\right)+\left(\chi_{b a^{k}}-\chi_{b a^{2 n-k}}+\chi_{b a^{n+k}}-\chi_{b a^{n-k}}\right)\right] \\
E_{2,1}^{k} & =\frac{1}{2}\left[-\left(-\chi_{a^{k}}+\chi_{a^{2 n-k}}+\chi_{a^{n+k}}-\chi_{a^{n-k}}\right)+\left(\chi_{b a^{k}}-\chi_{b a^{2 n-k}}+\chi_{b a^{n+k}}-\chi_{b a^{n-k}}\right)\right] \\
E_{1,1}^{n-k} & =\chi_{a^{n-k}}+\chi_{a^{2 n-k}} \\
E_{2,2}^{n-k} & =\chi_{a^{k}}+\chi_{a^{n+k}} \\
E_{1,2}^{n-k} & =\frac{1}{2}\left[\left(-\chi_{a^{k}}-\chi_{a^{2 n-k}}+\chi_{a^{n+k}}+\chi_{a^{n-k}}\right)+\left(\chi_{b a^{k}}-\chi_{b a^{2 n-k}}-\chi_{b a^{n+k}}+\chi_{b a^{n-k}}\right)\right] \\
E_{2,1}^{n-k} & =\frac{1}{2}\left[\left(-\chi_{a^{k}}-\chi_{a^{2 n-k}}+\chi_{a^{n+k}}+\chi_{a^{n-k}}\right)-\left(\chi_{b a^{k}}-\chi_{b a^{2 n-k}}-\chi_{b a^{n+k}}+\chi_{b a^{n-k}}\right)\right]
\end{aligned}
$$

Then,

$$
\begin{aligned}
& \psi\left(e_{1}\right)=\chi_{1}, \quad \psi\left(e_{2}\right)=\chi_{a^{n}}, \quad \psi\left(e_{3}\right)=\chi_{b a^{n}}, \quad \psi\left(e_{4}\right)=\chi_{b}, \\
& \psi\left(r_{i, j}^{k}\right)=E_{i, j}^{k}, \quad \psi\left(e_{i, j}^{n-k}\right)=E_{i, j}^{n-k} .
\end{aligned}
$$

8.4. The lattice $1(K Q(n))$ for $n$ odd. In the sequel of this section, we prove the following theorem, and illustrate it on $K Q(3)$.

Theorem 8.4. When $n$ is odd, $K Q(n)$ admits:

- A single coideal subalgebra of dimension $2 n: K_{2}^{\prime}=I\left(e_{1}+e_{2}\right)$, isomorphic to $L^{\infty}\left(D_{n}\right)$;

- $n$ coideal subalgebras of dimension $n$ in $K_{2}^{\prime}$;

- $n$ coideal subalgebras of dimension $4, J_{k}$ for $k=0, \ldots, n-1$, with Jones projections:

$$
p_{J_{k}}=e_{1}+\sum_{j=1, j \text { even }}^{n-1} q\left(0, \frac{j k \pi}{n}, j\right) ;
$$

- A single coideal subalgebra of dimension 2: $I\left(e_{1}+e_{2}+q_{1}+q_{2}\right)$.

If $n$ is prime there are no other coideal subalgebras and the graph of the lattice of coideal subalgebras of $K Q(n)$ is similar to Figure 14.

8.4.1. The coideal subalgebras of dimension 2 and $2 n$. From 8.1 .2 and $2.8 .6, \widehat{K Q(n)}$ has a single coideal subalgebra of dimension 2 . Therefore $K Q(n)$ has a single coideal subalgebra of dimension $2 n$.

By Remark 2.8.4 its standard coproduct is

$$
\Delta_{s}\left(e_{1}+e_{2}\right)=\left(e_{1}+e_{2}\right) \otimes\left(e_{1}+e_{2}\right)+\left(e_{3}+e_{4}\right) \otimes\left(e_{3}+e_{4}\right)+\sum_{j=1}^{n-1} e_{1,1}^{j} \otimes e_{2,2}^{j}+e_{2,2}^{j} \otimes e_{1,1}^{j} .
$$


Beware that, for an improved consistency with $K D(n)$, our notations differ slightly from those of [Vai98]:

$$
r_{1}=\frac{1}{2} \sum_{j=1, j \text { odd }}^{n-1} r_{1,1}^{j}, \quad r_{2}=\frac{1}{2} \sum_{j=1, j \text { odd }}^{n-1} r_{2,2}^{j},
$$

the unitary $\Omega^{\prime}$ used to twist the coproduct of $K Q(n)$ can be written as:

$$
\begin{aligned}
\Omega^{\prime} & =\left(e_{1}+e_{4}+r_{1}+q_{1}\right) \otimes\left(e_{1}+e_{4}+r_{1}+q_{1}\right) \\
& +\left(e_{1}+q_{1}\right) \otimes\left(e_{2}+e_{3}+r_{2}+q_{2}\right)+\left(e_{2}+e_{3}+r_{2}+q_{2}\right) \otimes\left(e_{1}+q_{1}\right) \\
& +\mathrm{i}\left(\mathrm{e}_{4}+\mathrm{r}_{1}\right) \otimes\left(\mathrm{e}_{2}-\mathrm{e}_{3}+\mathrm{q}_{2}-\mathrm{r}_{2}\right)-\mathrm{i}\left(\mathrm{e}_{2}-\mathrm{e}_{3}+\mathrm{q}_{2}-\mathrm{r}_{2}\right) \otimes\left(\mathrm{e}_{4}+\mathrm{r}_{1}\right) \\
& +\left(e_{2}-\mathrm{ie}_{3}+\mathrm{q}_{2}-\mathrm{ir}_{2}\right) \otimes\left(\mathrm{e}_{2}+\mathrm{ie}_{3}+\mathrm{q}_{2}+\mathrm{ir}_{2}\right) .
\end{aligned}
$$

Therefore:

$$
\begin{aligned}
\Delta\left(e_{1}+e_{2}\right)=\left(e_{1}+e_{2}\right) \otimes\left(e_{1}+e_{2}\right)+\left(e_{3}+e_{4}\right) \otimes\left(e_{3}+e_{4}\right) \\
+\sum_{j=1, j \text { even }}^{n-1} e_{1,1}^{j} \otimes e_{2,2}^{j}+e_{2,2}^{j} \otimes e_{1,1}^{j}+\sum_{j=1, j \text { odd }}^{n-1} p_{1,1}^{j} \otimes p_{1,1}^{j}+p_{2,2}^{j} \otimes p_{2,2}^{j} .
\end{aligned}
$$

The unique coideal subalgebra of dimension $2 n$ of $K Q(n)$ is therefore $K_{2}^{\prime}=I\left(e_{1}+e_{2}\right)$. As in 5.7 it can be shown to be isomorphic to $L^{\infty}\left(D_{n}\right)$. In particular, it admits $n$ coideal subalgebras of dimension $n$.

\subsubsection{The coideal subalgebras of dimension 4 and $n$.}

Proposition 8.4.2. For $n$ odd, the coideal subalgebras of dimension $n$ of $K Q(n)$ are contained in $K_{2}^{\prime}$. They correspond to the $n$ sub-groups of order 2 of $D_{n}$. By self-duality, $K Q(n)$ admits $n$ coideal subalgebras of dimension 4, with Jones projections:

$$
p_{J_{k}}=e_{1}+\sum_{j=1, j \text { even }}^{n-1} q\left(0, \frac{j k \pi}{n}, j\right) \quad(k=0, \ldots, n-1) .
$$

The principal graph of the inclusion $N_{1} \subset N_{1} \rtimes \delta\left(J_{k}\right)$ is $D_{n} / \mathbb{Z}_{2}$ (see 4.1), that of $N_{2} \subset$ $N_{2} \rtimes J_{k}$ is $D_{2 n+2}^{(1)}$ (see 4.3).

Proof. If $J$ is a coideal subalgebra of dimension $n$, its Jones projection is of the same form as described in 5.10 for $K D(n)$. From the classification of subfactors of index 4 in Pop90], the principal graph of $R \subset R \rtimes \delta^{-1}(J)$ is either $D_{4}^{(1)}$ or $D_{n^{\prime}}^{(1)}$. Therefore, $R \subset R \rtimes \delta^{-1}(J)$ admits an intermediate factor of index 2 , which implies that $J$ is contained in the unique coideal subalgebra $K_{2}^{\prime}$ of dimension $2 n$. Using 8.4.1, there are exactly $n$ coideal subalgebras of dimension $n$.

Since $K Q(n)$ is self-dual, it admits exactly $n$ coideal subalgebras of dimension 4 . As in 6.7, one shows that their Jones projections are, for $k=0, \ldots, n-1$ :

$$
p_{J_{k}}=e_{1}+\sum_{j=1, j \text { even }}^{n-1} q\left(0, \frac{j k \pi}{n}, j\right) .
$$

8.4.3. The Kac algebra $K Q(3)$ of dimension 12. We illustrate Theorem 8.4 on $K Q(3)$, making explicit the block matrix decompositions of the coideal subalgebras (obtained by computer) and the principal graphs of the corresponding inclusions. 


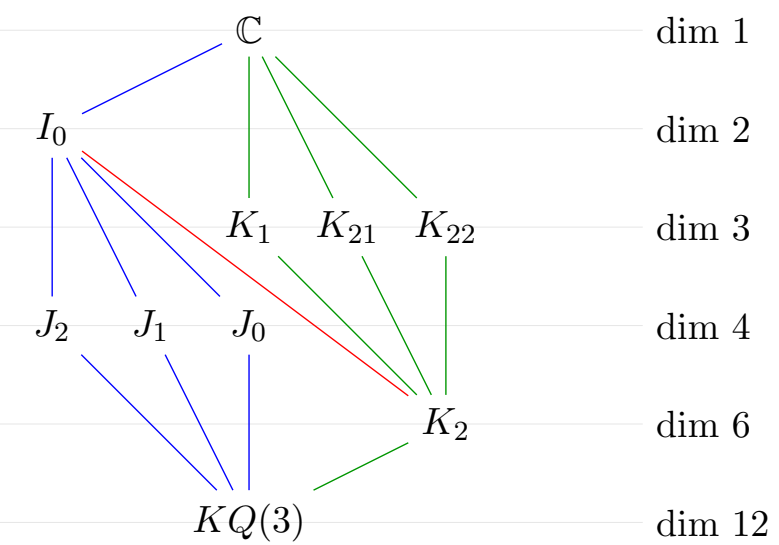

FIgURE 14. The lattice of coideal subalgebras of $K Q(3)$

Proposition 8.4.3. The lattice of coideal subalgebras de $K Q(3)$ is as given by Figure 14. The block matrix decomposition of the coideal subalgebras is given by:

$$
\begin{aligned}
I_{0} & =\mathbb{C}\left(e_{1}+e_{2}+q_{1}+q_{2}\right) \oplus \mathbb{C}\left(e_{3}+e_{4}+p_{1}+p_{2}\right) \\
K_{1} & =\mathbb{C}\left(e_{1}+e_{2}+e_{3}+e_{4}\right) \oplus \mathbb{C}\left(p_{1,1}^{1}+e_{2,2}^{2}\right) \oplus \mathbb{C}\left(p_{2,2}^{1}+e_{1,1}^{2}\right) ; \\
K_{21} & =\mathbb{C}\left(e_{1}+e_{2}+p_{1,1}^{1}\right) \oplus \mathbb{C}\left(e_{3}+e_{4}+e_{1,1}^{2}\right) \oplus \mathbb{C}\left(p_{2,2}^{1}+e_{2,2}^{2}\right) ; \\
K_{22} & =\mathbb{C}\left(e_{1}+e_{2}+p_{2,2}^{1}\right) \oplus \mathbb{C}\left(e_{3}+e_{4}+e_{2,2}^{2}\right) \oplus \mathbb{C}\left(p_{1,1}^{1}+e_{1,1}^{2}\right) ; \\
J_{0} & =\mathbb{C}\left(e_{1}+q_{1}\right) \oplus \mathbb{C}\left(e_{2}+q_{2}\right) \oplus \mathbb{C}\left(e_{3}+r_{2,2}^{1}\right) \oplus \mathbb{C}\left(e_{4}+r_{1,1}^{1}\right) ; \\
J_{1} & =\mathbb{C}\left(e_{1}+q\left(0, \frac{2 \pi}{3}, 2\right)\right) \oplus \mathbb{C}\left(e_{2}+q\left(0, \frac{5 \pi}{3}, 2\right)\right) \oplus \mathbb{C}\left(e_{3}+q\left(\frac{\pi}{3}, \frac{\pi}{2}, 1\right)\right) \oplus \mathbb{C}\left(e_{4}+q\left(-\frac{\pi}{3}, \frac{3 \pi}{2}, 1\right)\right) ; \\
J_{2} & =\mathbb{C}\left(e_{1}+q\left(0, \frac{4 \pi}{3}, 2\right)\right) \oplus \mathbb{C}\left(e_{2}+q\left(0, \frac{\pi}{3}, 2\right)\right) \oplus \mathbb{C}\left(e_{3}+q\left(-\frac{\pi}{3}, \frac{\pi}{2}, 1\right)\right) \oplus \mathbb{C}\left(e_{4}+q\left(\frac{\pi}{3}, \frac{3 \pi}{2}, 1\right)\right) ; \\
K_{2} & =I\left(e_{1}+e_{2}\right)=\mathbb{C}\left(e_{1}+e_{2}\right) \oplus \mathbb{C}\left(e_{3}+e_{4}\right) \oplus \mathbb{C} p_{1,1}^{1} \oplus \mathbb{C} p_{2,2}^{1} \oplus \mathbb{C} e_{1,1}^{2} \oplus \mathbb{C} e_{2,2}^{2} .
\end{aligned}
$$

With the notations of 4 , the inclusion $N_{2} \subset N_{2} \rtimes J$ has for principal graph

- $D_{8}^{(1)}$ for $J=J_{1}$, or $J_{2}$;

- $A_{5}$ for $J=K_{1}, K_{21}$, or $K_{22}$.

For $J=I_{0}, J_{0}$, or $K_{2}$, it is of depth 2 . 


\section{The KaC algebras $K B(n)$ and $K_{3}$ In $K D(2 m)$}

When $n=2 m$ is even, the Kac subalgebras $K_{2}$ and $K_{4}$ of $K D(n)$ are respectively isomorphic to $L^{\infty}\left(D_{m}\right)$ and $K D(m)$; their structure is therefore known, or can at least be studied recursively. There remains to describe the family of Kac subalgebras $K_{3}$, as $m$ varies. This question was actually at the origin of our interest in the family of Kac algebras $K Q(n)$, and later in the family $B_{4 m}$ defined by A. Masuoka in [Mas00, def 3.3], and which we denote $K B(m)$ for notational consistency.

In this section, we first prove that, for all $m, K_{3}$ in $K D(2 m)$ is isomorphic to $K B(m)$, and therefore self-dual, and discuss briefly a construction of $K_{3}$ by composition of factors. Then, we compare the three families: $K_{3}$ in $K D(n)$ is also isomorphic to $K Q(m)$ when $m$ is odd (this gives an alternative proof of the self-duality of $K Q(m)$ when $m$ is odd) and the family $(K D(n))_{n}$ contains the others, $(K Q(n))_{n}$ and $(K B(n))_{n}$, and $K P$ as subalgebras. Using the self-duality of $K B(m)$, we list the coideal subalgebras of dimension 2, 4, $m$, and $2 m$ of $K_{3}$ in $K D(2 m)$ and describe the complete lattice of coideal subalgebras for $K D(8)$.

9.1. The subalgebra $K_{3}$ of $K D(2 m)$. In $K D(4), K_{3}$ is isomorphic to $K P$ (see 7.6); in particular, it is self-dual and can be obtained by twisting a group algebra. In $K D(8)$, $K_{3}$ is the self-dual Kac algebra described in [IK02, Lemma XI.10] (the intrinsic group of its dual is $\mathbb{Z}_{2} \times \mathbb{Z}_{2}$ ). We however found on computer that it cannot be obtained by twisting a group algebra: we took a generic cocycle $\omega$ on $H$ with six unknowns such that $\omega(h, h)=1$, and $\omega\left(h, h^{\prime}\right)=\alpha_{h, h^{\prime}}$ if $h \neq h^{\prime}$, and asked whether there existed a choice for the $\alpha_{h, h^{\prime}}$ making the untwisted coproduct $\Delta_{u}=\Omega \Delta \Omega^{*}$ is cocommutative; this gave an algebraic system of equations of degree 2 which had no solution (note that we relaxed the condition $\omega\left(h, h^{\prime}\right)=\overline{\omega\left(h^{\prime}, h\right)}$ to keep the system algebraic).

9.1.1. A presentation of $K_{3}$ and isomorphism with $K B(m)$. We now construct explicit generators for $K_{3}$ satisfying the presentation of $K B(m)$ given in [Mas00, def 3.3].

Lemma 9.1.1. With $n=2 m$ and $\epsilon=\mathrm{e}^{\mathrm{i} \pi /(2 \mathrm{~m})}$ set:

$$
\begin{aligned}
v & =\left(e_{1}+e_{3}\right)-\left(e_{2}+e_{4}\right)+\sum_{j=1}^{m-1} \epsilon^{-2 j} e_{1,2}^{2 j}+\epsilon^{2 j} e_{2,1}^{2 j}+\sum_{j=0}^{m-1} \epsilon^{-(2 j+1)} r_{1,2}^{2 j+1}+\epsilon^{2 j+1} r_{2,1}^{2 j+1} \\
w & =\left(e_{1}+e_{3}\right)-\left(e_{2}+e_{4}\right)+\sum_{j=1}^{m-1} \epsilon^{2 j} e_{1,2}^{2 j}+\epsilon^{-2 j} e_{2,1}^{2 j}+\sum_{j=0}^{m-1} \epsilon^{2 j+1} r_{1,2}^{2 j+1}+\epsilon^{-(2 j+1)} r_{2,1}^{2 j+1} \\
B_{0} & =\left(1+\lambda\left(a^{n}\right)\right) / 2 \\
B_{1} & \left.=\left(1-\lambda\left(a^{n}\right)\right) / 2\right)
\end{aligned}
$$

The unitary elements $v$ and $w$ are contained in $K_{3}$ and satisfy:

$$
\begin{gathered}
v^{2}=w^{2}=1 \\
(v w)^{m}=\lambda\left(a^{n}\right) \\
\Delta(v)=v \otimes B_{0} v+w \otimes B_{1} v \quad \text { et } \quad \Delta(w)=w \otimes B_{0} w+v \otimes B_{1} w \\
\varepsilon(v)=\varepsilon(w)=1 \\
S(v)=B_{0} v+B_{1} w \quad \text { et } \quad S(w)=B_{0} w+B_{1} v
\end{gathered}
$$

Proof. The non trivial part is the calculation of the coproducts which is carried over in A.6.

Theorem 9.1.1. The Kac subalgebra $K_{3}$ in $K D(2 m)$ is isomorphic to the Kac algebra $K B(m)$ defined by A. Masuoka in [Mas00, def 3.3]. In particular, it is self-dual. 
Proof. Using Lemma 9.1.1, $K_{3}$ contains the subalgebra $I_{0}=\mathbb{C Z}_{2}$ generated by $\lambda\left(a^{n}\right)$, as well as the unitary elements $v$ and $w$ which satisfy the desired relations. By dimension count, $K_{3}$ is therefore isomorphic to $K B(m)$. The later is self-dual (CDMM04, p. 776]).

9.1.2. Realization of $K_{3}$ by composition of subfactors. As in 3.4 and 6.9.2, we describe the inclusion $N_{2} \subset N_{2} \rtimes K_{3}$ using group actions.

Define the group $G=D_{m} \rtimes \mathbb{Z}_{2}$, where $\mathbb{Z}_{2}$ acts on $D_{m}=\langle\alpha, \beta| \alpha^{m}=1, \beta^{2}=1, \beta \alpha=$ $\left.\alpha^{-1} \beta\right\rangle$ by the automorphism $\nu$ of $D_{m}$ defined by $\nu(\alpha)=\alpha^{-1}$ and $\nu(\beta)=\alpha \beta$. Set $M=$ $N_{2} \rtimes K_{1}$. Consider the dual action of $D_{m}$ on $M\left(K_{1}\right.$ is isomorphic to $\left.L^{\infty}\left(D_{m}\right)\right)$ and the action of $z$ of $\mathbb{Z}_{2}$ on $M$ by $\operatorname{Ad}(v)$. As in [IK02, def II.1], it can be easily proved that $(d, z)$ is an outer action of the matched pair $\left(D_{m}, \mathbb{Z}_{2}\right)$. From $(d, z)$ arises a couple of cocycles $(\eta, \zeta)$ whose class in a certain cohomology group $H^{2}\left(\left(D_{m}, \mathbb{Z}_{2}\right), T\right)$ characterizes up to an isomorphism the depth 2 irreducible inclusion of factors $M^{\left(D_{m}, d\right)} \subset M \rtimes_{z} \mathbb{Z}_{2}$ ([IK02, Theorem II.5 and Remark 2 p.12]) and the Kac algebra which is associated to this inclusion ([IK02, Theorem VI.1]). The group $H^{2}\left(\left(D_{m}, \mathbb{Z}_{2}\right), T\right)$ is reduced to $\mathbb{Z}_{2}$ ([IK02, VII. $\S 5$ and Proposition VII.5]) and the pair $(d, z)$ is associated to the non-trivial cocycle. Indeed, if $m$ is even, this follows from [IK02, Lemma VII.6], since the intrinsic group of $K_{3}$ and $\widehat{K}_{3}$ are of order 4 (that of $K_{3}$ is $J_{20} \equiv \mathbb{Z}_{2} \times \mathbb{Z}_{2}$ and $K_{3}$ has four characters). If $m$ is odd, $K_{3}$ is isomorphic to $K Q(m)$, which is self-dual, and its intrinsic group is $\mathbb{Z}_{4}$, so one can use [IK02, Lemma VII.2]. For any value of $m$, the inclusion $N_{2} \subset N_{2} \rtimes K_{3}$ is therefore isomorphic to the inclusion $M^{\left(D_{m}, d\right)} \subset M \rtimes_{z} \mathbb{Z}_{2}$ associated to the non trivial cocycle.

9.2. $K D(n), K Q(n), K A(n)$, and $K B(n)$ : isomorphisms, coideal subalgebras of dimension $2 n$. We collect here all the results on the isomorphisms between the four families and their Kac subalgebras of dimension $2 n$.

Theorem 9.2. Let $n \geq 2$.

(1) The Kac algebra $K A(n)$ is isomorphic to the dual of $K D(n)$.

(2) Assume further $n=2 m$ even. Then, in $K D(n)$,

- $K_{2}=I\left(e_{1}+e_{2}\right)$ is isomorphic to $L^{\infty}\left(D_{n}\right)$,

- $K_{3}=I\left(e_{1}+e_{3}\right)$ is isomorphic to $K B(m)$,

- $K_{4}=I\left(e_{1}+e_{4}\right)$ is isomorphic to $K D(m)$.

(3) The Kac algebra $K Q(n)$ is isomorphic to $K D(n)$ for $n$ even and to $K B(n)$ for $n$ odd.

Proof. (1) For $n=2$, see [Mas00, Remark 3.4 (1)]. For $n \geq 3, K A(n)$ is the unique non trivial Kac algebra obtained by twisting the product of $L^{\infty}\left(D_{n}\right)$ by a cocycle (see [Mas00, Theorem $4.1(2)]$ ). Its dual is therefore the unique non trivial Kac algebra obtained by twisting the coproduct of $\mathbb{C}\left[D_{n} 9\right.$. Hence, $K A(n)$ must be isomorphic to $K D(n)$.

(2) See Proposition 5.7, Theorem 9.1.1, Theorem 5.4.2 for the structure of $K_{2}, K_{3}$, and $K_{4}$ respectively.

(3) For $n$ even, $K Q(n)$ is isomorphic to $K D(n)$ (Theorem 8.2). If $n$ odd, $K Q(n)$, which is embedded in $K Q(2 n)$ as $K_{3}^{\prime}$, is isomorphic to $K_{3}$ of $K D(2 n)$ by Proposition 8.2.3, and therefore to $K B(n)$ by Theorem 9.1.1.

\subsection{The lattice $1(K B(m))$.}

\footnotetext{
${ }^{9}$ It is a classical fact in the folklore that twisting products or coproducts of algebras by cocycles are two dual constructions
} 
9.3.1. General case. In 8.4, we partially describe the lattice $1(K Q(m))$ when $m$ is odd; those results therefore apply to the isomorphic algebras $K B(m)$ and $K_{3}$ in $K D(2 m)$.

Let us now explore $1(K B(m))$ for $m$ even: $m=2 m^{\prime}$. To this end, we consider $K B(m)$ as $K_{3}$ in $K D(2 m)$. To avoid handling degeneracies, the special cases $K B(2)=K P$ and $K B(4)$ are treated respectively in Subsections 3.3 and 9.3 .2 , and we now assume $m \geq 6$.

Proposition 9.3.1. When $m$ is even, the coideal subalgebras of dimension $2,4, m, 2 m$ of the Kac algebra $K B(m) \approx K_{3} \subset K D(2 m)$ are, keeping the notations of 7.1.2 and 7.5:

- Three coideal subalgebras of dimension $2 m$ :

$-K_{32}=K_{1}=I\left(e_{1}+e_{2}+e_{3}+e_{4}\right)$, isomorphic to $L^{\infty}\left(D_{m}\right)$;

$-K_{33}=I\left(e_{1}+e_{3}+r_{1,1}^{m}\right)=I\left(p_{\left\langle a^{4}, b a\right\rangle}\right)$;

$-K_{34}=I\left(e_{1}+e_{3}+r_{2,2}^{m}\right)=I\left(p_{\left\langle a^{4}, a b\right\rangle}\right)$;

The coideal subalgebras $K_{33}$ and $K_{34}$ are isomorphic through the involutive automorphism $\Theta_{-1}$;

- The coideal subalgebras of $K_{32}$, which correspond to subgroups of $D_{m}$; in dimension 2, 4, $m$, they are:

- Dimension 2: $I_{0}, I_{3}$, and $I_{4}$ (subgroups of order $m$ );

- Dimension 4: the coideal subalgebra $J_{20}$ containing $I_{0}, I_{3}$, and $I_{4}$; furthermore, if $m^{\prime}$ is even, there are four coideal subalgebras (dihedral subgroups of order $\left.m^{\prime}\right)$, which contain exactly one of $I_{3}$ or $I_{4}$;

- Dimension $m: K_{31}=I\left(e_{1}+e_{2}+e_{3}+e_{4}+e_{1,1}^{m}+e_{2,2}^{m}\right)$ (subgroup generated by $\left.\alpha^{m^{\prime}}\right)$, and $K_{1 k}=I\left(e_{1}+e_{2}+e_{3}+e_{4}+r_{1,1}^{2 k-1}+r_{2,2}^{n-2 k+1}\right)$ for $k=1, \ldots, m$ (subgroups generated by one reflection); each $K_{1 k}$ contains exactly one of $I_{3}$ or $I_{4}$

- The coideal subalgebras of $K_{33}$ and $K_{34}$ :

- Dimension 2: $I_{0}$, and if $m^{\prime}$ is even, $I_{3}$ and $I_{4}$;

- Dimension 4: $J_{2 k+1}$ for $k=0, \ldots m-1$ and, if $m^{\prime}$ is even, $J_{20}$; for $2 k+1 \equiv 1$ (resp. $2 k+1 \equiv 3) \bmod 4, J_{2 k+1}$ is contained in $K_{33}$ (resp. $\left.K_{34}\right)$;

- Dimension $m: K_{31}$, and if $m^{\prime}$ is even, two coideal subalgebras contained in $K_{33}$ and two in $K_{34}$.

In particular, $K_{3}$ admits exactly $m$ coideal subalgebras of dimension 4 not contained in $K_{1}$. Also, $K_{31}$ is the intersection of the three coideal subalgebras of dimension $2 m$ of $K_{3}$.

Proof. Since $K_{32}=K_{1}=L^{\infty}\left(D_{m}\right)$ the description of its lattice of coideal subalgebras is straightforward.

From 7.1.2 and 7.5, we know that $K_{3}$ admits three coideal subalgebras of dimension 2: $I_{0}, I_{3}$, and $I_{4}$. By self-duality of $K_{3}$, there are exactly three coideal subalgebras of dimension $2 m$, and by trace argument the given Jones projections for $K_{32}, K_{33}$, and $K_{34}$ are the only possible ones. Their expressions in the group basis is straightforward.

Then, any automorphism of $K D(2 m)$ stabilizes $K_{3}$ and therefore induces an automorphism of $K_{3}$. From the expressions of the Jones projection in the group basis, the involutive automorphism $\Theta_{-1}$ exchanges $K_{33}$ and $K_{34}$ which are therefore isomorphic.

The specified inclusions are derived by comparison of the Jones projections. Looking at the inclusion diagrams imposes that $\delta$ maps $I_{0}$ to $K_{1}, I_{3}$ and $I_{4}$ to $K_{33}$ and $K_{34}$ (or $K_{34}$ and $K_{33}$ ), and $J_{20}$ to $K_{31}$, and the $J_{2 k+1}$ to the $K_{1 h}$ (see 7.5).

As in 5.10, the coideal subalgebras of dimension dividing $2 m$ are either contained in $K_{1}$ or in exactly one of $K_{33}$ or $K_{34}$. Therefore, the remaining coideal subalgebras of dimension 4 and $m$ of $K_{3}$ are in exactly one of $K_{33}$ or $K_{34}$. As $K_{33}$ ad $K_{34}$ are isomorphic, it is sufficient to find those in $K_{33}$. Any coideal subalgebra of dimension 4 contains a coideal subalgebra of dimension 2, since it is the image by $\delta$ of a coideal subalgebra of dimension $m$ contained in one of dimensions $2 m$. The next argument depends on the parity of $m^{\prime}$ : 
If $m^{\prime}$ is odd, the only coideal subalgebra of dimension 2 contained in $K_{33}$ is $I_{0}$; therefore the coideal subalgebras of dimension 4 of $K_{33}$ are mapped by $\delta$ on the $m+1$ coideal subalgebras of dimension $m$ of $K_{1}$ : they are $J_{20}$ and the $J_{2 k+1}$.

If $m^{\prime}$ is even, $K_{33}$ admits only 4 projections of trace $1 / 2 m$ (see A.7); therefore, $K_{33}$ contains at most 3 coideal subalgebras of dimension $m$, including $K_{31}$. Conclusion: $K_{3}$ contains at most $m+5$ coideal subalgebras of dimension $m$ and at most $m+5$ coideal subalgebras of dimension 4 , and by self-duality it contains exactly $m+5$ coideal subalgebras de dimension $m$ and $m+5$ coideal subalgebras de dimension 4 .

In both cases, $K_{3}$ admits exactly $m$ coideal subalgebras of dimension 4 not contained in $K_{1}$.

9.3.2. Lattice $\mathrm{l}(K D(8))$. Since $n$ is a power of 2 , any coideal subalgebra is contained in one of the $K_{i}$ 's (see prop 5.10). The lattice $1(K D(8))$ can therefore be constructed from those of $K_{2} \equiv L^{\infty}\left(D_{8}\right), K_{3}$, and $K_{4} \equiv K D(4)$ (see 7.6)

Proposition 9.3.2. The lattice of coideal subalgebras of $K D(8)$ is as given in Figure 15 .

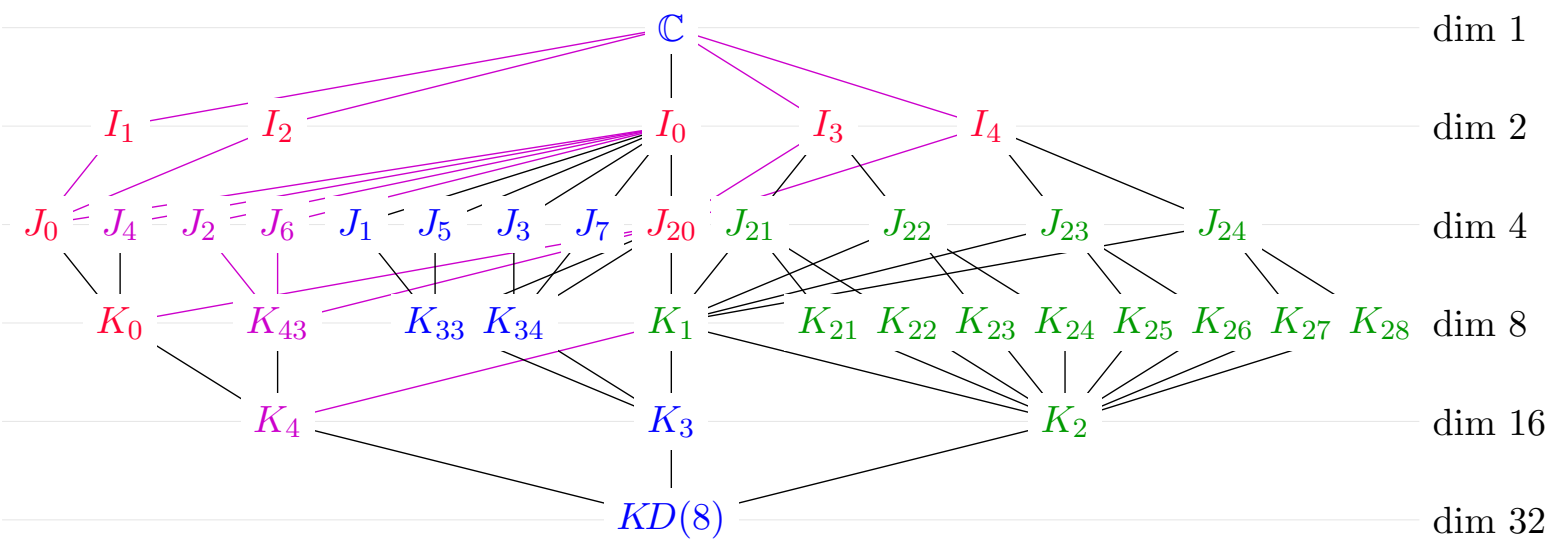

Figure 15. The lattice of coideal subalgebras of $K D(8)$

Proof. We proceed essentially as in the general case. The algebra $K_{1}$ admits, beside $J_{20}$, four coideal subalgebras of dimension $m=4$, denoted $J_{21}, J_{22}, J_{23}$, and $J_{24}$ which each contains either $I_{3}$ or $I_{4}$. Beside $K_{1}$, there are two coideal subalgebras de dimension 8:

- $K_{33}=I\left(e_{1}+e_{3}+r_{1,1}^{4}\right)$ containing $J_{20}, J_{1}$, and $J_{5}$;

- $K_{34}=I\left(e_{1}+e_{3}+r_{2,2}^{4}\right)$ containing $J_{20}, J_{3}$, and $J_{7}$.

Both $K_{33}$ and $K_{34}$ have $\mathbb{C} \oplus \mathbb{C} \oplus \mathbb{C} \oplus \mathbb{C} \oplus M_{2}(\mathbb{C})$ as matrix structure (computer calculation). Therefore they both admit, beside $J_{20}$, exactly two coideal subalgebras of dimension 4; those are $J_{1}, J_{5}$ and $J_{3}, J_{7}$. Their images by $\delta$ of $K_{3}$ are $J_{21}, J_{22}$ and $J_{23}, J_{24}$. 


\section{Appendix A. Collection of formulas for $K D(n)$}

In this appendix, we collect all the formulas used in the study of the algebra $K D(n)$ : left regular representation and expression of matrix units in the group basis, standard coproducts of some special elements, expression of $\Omega$ in terms of matrix units, method to calculate the twisted coproducts and its expression on some elements.

\section{A.1. Left regular representation of $D_{2 n}$. Vai98, 6.6]}

Set $\epsilon_{n}=\mathrm{e}^{i \pi / n}$. The left regular representation of $D_{2 n}$ in terms of matrix units is given by, for all $k$ :

$$
\begin{gathered}
\lambda\left(a^{k}\right)=e_{1}+e_{2}+(-1)^{k}\left(e_{3}+e_{4}\right)+\sum_{j=1}^{n-1}\left(\epsilon_{n}^{j k} e_{1,1}^{j}+\epsilon_{n}^{-j k} e_{2,2}^{j}\right) \\
\lambda\left(b a^{k}\right)=e_{1}-e_{2}-(-1)^{k}\left(e_{3}-e_{4}\right)+\sum_{j=1}^{n-1}\left(\epsilon_{n}^{-j k} e_{1,2}^{j}+\epsilon_{n}^{j k} e_{2,1}^{j}\right)
\end{gathered}
$$

A.2. Matrix units in the group basis and coinvolution. By inverting the previous formulas we get the following expressions for the matrix units in the group basis:

$$
\begin{array}{llrl}
e_{1} & =\frac{1}{4 n} \sum_{k=0}^{2 n-1}\left(\lambda\left(a^{k}\right)+\lambda\left(b a^{k}\right)\right), & e_{2} & =\frac{1}{4 n} \sum_{k=0}^{2 n-1}\left(\lambda\left(a^{k}\right)-\lambda\left(b a^{k}\right)\right), \\
e_{3} & =\frac{1}{4 n} \sum_{k=0}^{2 n-1}(-1)^{k}\left(\lambda\left(a^{k}\right)-\lambda\left(b a^{k}\right)\right), & e_{4} & =\frac{1}{4 n} \sum_{k=0}^{2 n-1}(-1)^{k}\left(\lambda\left(a^{k}\right)+\lambda\left(b a^{k}\right)\right), \\
e_{1,1}^{j} & =\frac{1}{2 n} \sum_{k=0}^{2 n-1} \mathrm{e}^{-i j k \pi / n} \lambda\left(a^{k}\right), & e_{2,2}^{j} & =\frac{1}{2 n} \sum_{k=0}^{2 n-1} \mathrm{e}^{i j k \pi / n} \lambda\left(a^{k}\right), \\
e_{1,2}^{j} & =\frac{1}{2 n} \sum_{k=0}^{2 n-1} \mathrm{e}^{i j k \pi / n} \lambda\left(b a^{k}\right), & e_{2,1}^{j} & =\frac{1}{2 n} \sum_{k=0}^{2 n-1} \mathrm{e}^{-i j k \pi / n} \lambda\left(b a^{k}\right) .
\end{array}
$$

For the convenience of the reader, here are some direct consequences of those formulas:

$$
\begin{array}{rlrl}
e_{1}+e_{2} & =\frac{1}{2 n} \sum_{k=0}^{2 n-1} \lambda\left(a^{k}\right), & e_{3}+e_{4} & =\frac{1}{2 n} \sum_{k=0}^{2 n-1}(-1)^{k} \lambda\left(a^{k}\right), \\
e_{1}+e_{3}=\frac{1}{2 n} \sum_{k=0}^{n-1} \lambda\left(a^{2 k}\right)+\lambda\left(b a^{2 k+1}\right), & e_{1}+e_{4}=\frac{1}{2 n} \sum_{k=0}^{n-1} \lambda\left(a^{2 k}\right)+\lambda\left(b a^{2 k}\right), \\
e_{2}+e_{3}=\frac{1}{2 n} \sum_{k=0}^{n-1}\left(\lambda\left(a^{2 k}\right)-\lambda\left(b a^{2 k}\right)\right), & e_{1}+e_{2}+e_{3}+e_{4}=\frac{1}{n} \sum_{k=0}^{n-1} \lambda\left(a^{2 k}\right), \\
e_{1,1}^{j}+e_{2,2}^{n-j}=\frac{1}{n} \sum_{k=0}^{n-1} \mathrm{e}^{-2 i j k \pi / n} \lambda\left(a^{2 k}\right), & e_{1,2}^{j}+e_{2,1}^{n-j}=\frac{1}{n} \sum_{k=0}^{n-1} \mathrm{e}^{2 i j k \pi / n} \lambda\left(b a^{2 k}\right), \\
e_{1,2}^{j}-e_{2,1}^{n-j}=\frac{1}{n} \sum_{k=0}^{n-1} \mathrm{e}^{i j(2 k+1) \pi / n} \lambda\left(b a^{2 k+1}\right) . &
\end{array}
$$

The coinvolution is the antiisomorphism defined by $S(\lambda(g))=\lambda(g)^{*}$. It fixes the $e_{i}$ and $e_{i, j}^{k}($ with $i \neq j)$ and exchanges the $e_{1,1}^{j}$ and $e_{2,2}^{j}$. 
A.3. The unitary 2-cocycle $\Omega$. In [Vai98, 6.6] the unitary 2-cocycle $\Omega$ used to twist the coproduct is expressed in the group basis:

$$
\Omega=\sum_{i, j=1}^{4} c_{i, j} \lambda\left(h_{i}\right) \otimes \lambda\left(h_{j}\right),
$$

where $h_{1}=1, h_{2}=a^{n}, h_{3}=b, h_{4}=b a^{n}$, and the $c_{i, j}$ are the coefficients of the matrix:

$$
\frac{1}{8}\left(\begin{array}{cccc}
5 & 1 & 1 & 1 \\
1 & 1 & \bar{\nu} & \nu \\
1 & \nu & 1 & \bar{\nu} \\
1 & \bar{\nu} & \nu & 1
\end{array}\right) \quad \text { with } \nu=-1+2 \mathrm{i}
$$

Furthermore, $\Omega^{*}=\sum_{i, j=1}^{4} c_{j, i} \lambda\left(h_{i}\right) \otimes \lambda\left(h_{j}\right)$.

We deduce its expression in terms of matrix units:

For $n$ even:

$$
\begin{aligned}
\Omega & =\left(e_{1}+e_{2}+e_{3}+e_{4}+q_{1}+q_{2}\right) \otimes\left(e_{1}+e_{2}+e_{3}+e_{4}+q_{1}+q_{2}\right) \\
& +\left(p_{1}+p_{2}\right) \otimes\left(e_{1}+e_{4}+q_{1}\right)+\left(e_{1}+e_{4}+q_{1}\right) \otimes\left(p_{1}+p_{2}\right) \\
& +i\left(p_{1}-p_{2}\right) \otimes\left(e_{2}+e_{3}+q_{2}\right)-i\left(e_{2}+e_{3}+q_{2}\right) \otimes\left(p_{1}-p_{2}\right)+\left(p_{1}+i p_{2}\right) \otimes\left(p_{1}-i p_{2}\right)
\end{aligned}
$$

For $n$ odd:

$$
\begin{aligned}
\Omega & =\left(e_{1}+e_{2}+q_{1}+q_{2}\right) \otimes\left(e_{1}+e_{2}+q_{1}+q_{2}\right) \\
& +\left(e_{3}+e_{4}+p_{1}+p_{2}\right) \otimes\left(e_{1}+q_{1}\right)+\left(e_{1}+q_{1}\right) \otimes\left(e_{3}+e_{4}+p_{1}+p_{2}\right) \\
& +i\left(e_{2}+q_{2}\right) \otimes\left(e_{3}-e_{4}-p_{1}+p_{2}\right)-i\left(e_{3}-e_{4}-p_{1}+p_{2}\right) \otimes\left(e_{2}+q_{2}\right) \\
& +\left(e_{3}-i e_{4}-i p_{1}+p_{2}\right) \otimes\left(e_{3}+i e_{4}+i p_{1}+p_{2}\right)
\end{aligned}
$$

Remark A.3. Note that the conjugation by $\Omega$ acts similarly on all factors $M_{2}(\mathbb{C})$ of same parity. For example, if $x$ and $y$ are each in some even factor (not necessarily the same), then:

$$
\Omega(x \otimes y) \Omega^{*}=\left(q_{1}+q_{2}\right) \otimes\left(q_{1}+q_{2}\right)(x \otimes y)\left(q_{1}+q_{2}\right) \otimes\left(q_{1}+q_{2}\right)=x \otimes y .
$$

If $x$ and $y$ are each in some odd factor (not necessarily the same), then:

$$
\Omega(x \otimes y) \Omega^{*}=\left(-i p_{1}+p_{2}\right) \otimes\left(i p_{1}+p_{2}\right)(x \otimes y)\left(i p_{1}+p_{2}\right) \otimes\left(-i p_{1}+p_{2}\right) .
$$

In particular, for $j$ and $j^{\prime}$ odd:

$$
\Omega\left(e_{1,2}^{j} \otimes e_{1,2}^{j^{\prime}}\right) \Omega^{*}=r_{2,1}^{j} \otimes r_{1,2}^{j^{\prime}} .
$$

In A.6, we shall see an example of twisting of $x \otimes y$ for $x$ in some odd factor and $y$ in some even factor. In subsequent sections, we give further examples of calculation of twisted coproducts using this remark.

A.4. Twisted coproduct of the Jones projection of the subgroup $H_{r}$. Recall that, in $\mathbb{C}\left[D_{2 n}\right]$ and for $r=1, \ldots, n-1$, the projection

$$
Q_{r}=\frac{1}{4}\left(1+\lambda\left(a^{n}\right)+\lambda\left(b a^{r}\right)+\lambda\left(b a^{r+n}\right)\right),
$$

is the Jones projection of the subalgebra $\mathbb{C}\left[H_{r}\right]$ of the subgroup

$$
H_{r}=\left\{1, \lambda\left(a^{n}\right), \lambda\left(b a^{r}\right), \lambda\left(b a^{r+n}\right)\right\} .
$$

In order to illustrate Remark A.3. we prove that the twisted coproduct of $Q_{r}$ is of the form

$$
\Delta\left(Q_{r}\right)=Q_{r} \otimes Q_{r}+Q_{r}^{\prime} \otimes Q_{r}^{\prime}+S\left(\tilde{P}_{r}\right) \otimes \tilde{P}_{r}+S\left(\tilde{P}_{r}^{\prime}\right) \otimes \tilde{P}_{r}^{\prime}
$$


where $\tilde{P}_{r}$ and $\tilde{P}^{\prime}{ }_{r}$ are defined below, depending on the parity of $n$. This is used in the proof of Proposition 5.11 to conclude that $Q_{r}$ is the Jones projection of $J_{r}$, spanned by $Q_{r}, Q_{r}^{\prime}, \tilde{P}_{r}$ and $\tilde{P}_{r}^{\prime}$.

We recall from 2.8 .4 that the standard coproduct of $Q_{r}$ is:

$$
\Delta_{s}\left(Q_{r}\right)=Q_{r} \otimes Q_{r}+Q_{r}^{\prime} \otimes Q_{r}^{\prime}+P_{r} \otimes P_{r}+P_{r}^{\prime} \otimes P_{r}^{\prime}
$$

where $Q_{r}, Q_{r}^{\prime}, P_{r}$ and $P_{r}^{\prime}$ are the minimal projections of $J_{r}$ (notice that all the elements of the group $H_{r}$ are of order 2).

Let us start with $n$ odd, and express $Q_{r}, Q_{r}^{\prime}, P_{r}, P_{r}^{\prime}$ in the matrix units basis:

$$
\begin{aligned}
& Q_{r}=\frac{1}{4}\left(1+\lambda\left(a^{n}\right)+\lambda\left(b a^{r}\right)+\lambda\left(b a^{r+n}\right)\right)=e_{1}+\sum_{j=1, j \text { even }}^{n-1} q\left(0, \frac{j r \pi}{n}, j\right) . \\
& Q_{r}^{\prime}=\frac{1}{4}\left(1+\lambda\left(a^{n}\right)-\lambda\left(b a^{r}\right)-\lambda\left(b a^{r+n}\right)\right)=e_{2}+\sum_{j=1, j \text { even }}^{n-1} q\left(0, \frac{j r \pi}{n}+\pi, j\right) .
\end{aligned}
$$

For $r$ even:

$$
\begin{aligned}
& P_{r}=\frac{1}{4}\left(1-\lambda\left(a^{n}\right)-\lambda\left(b a^{r}\right)+\lambda\left(b a^{r+n}\right)\right)=e_{3}+\sum_{j=1, j \text { odd }}^{n-1} q\left(0, \frac{j r \pi}{n}+\pi, j\right), \\
& P_{r}^{\prime}=\frac{1}{4}\left(1-\lambda\left(a^{n}\right)+\lambda\left(b a^{r}\right)-\lambda\left(b a^{r+n}\right)\right)=e_{4}+\sum_{j=1, j \text { odd }}^{n-1} q\left(0, \frac{j r \pi}{n}, j\right) .
\end{aligned}
$$

For $r$ odd:

$$
\begin{aligned}
& P_{r}=\frac{1}{4}\left(1-\lambda\left(a^{n}\right)-\lambda\left(b a^{r}\right)+\lambda\left(b a^{r+n}\right)\right)=e_{4}+\sum_{j=1, j \text { odd }}^{n-1} q\left(0, \frac{j r \pi}{n}+\pi, j\right), \\
& P_{r}^{\prime}=\frac{1}{4}\left(1-\lambda\left(a^{n}\right)+\lambda\left(b a^{r}\right)-\lambda\left(b a^{r+n}\right)\right)=e_{3}+\sum_{j=1, j \text { odd }}^{n-1} q\left(0, \frac{j r \pi}{n}, j\right) .
\end{aligned}
$$

Applying A.3, yields that:

$\Delta\left(Q_{r}\right)=Q_{r} \otimes Q_{r}+Q_{r}^{\prime} \otimes Q_{r}^{\prime}+\left(V \otimes V^{*}\right)\left(P_{r} \otimes P_{r}\right)\left(V^{*} \otimes V\right)+\left(V \otimes V^{*}\right)\left(P_{r}^{\prime} \otimes P_{r}^{\prime}\right)\left(V^{*} \otimes V\right)$, where $V=\left(e_{3}-i e_{4}-i p_{1}+p_{2}\right)$, and $\left(V \otimes V^{*}\right)$ is the unique term of $\Omega$ which acts non trivially. Note that the following relations hold:

$$
V e_{3} V^{*}=e_{3}, \quad V e_{4} V^{*}=e_{4}, \quad P_{r}+P_{r}^{\prime}=e_{3}+e_{4} .
$$

Therefore, it is sufficient to conjugate $q\left(0, \frac{j r \pi}{n}, j\right)$ with $\pm i p_{1}+p_{2}$. As desired, we get:

$$
\Delta\left(Q_{r}\right)=Q_{r} \otimes Q_{r}+Q_{r}^{\prime} \otimes Q_{r}^{\prime}+S\left(\tilde{P}_{r}\right) \otimes \tilde{P}_{r}+S\left(\tilde{P}_{r}^{\prime}\right) \otimes \tilde{P}_{r}^{\prime},
$$

with, for $r$ even:

$$
\tilde{P}_{r}=e_{3}+\sum_{j=1, j \text { odd }}^{n-1} q\left(-\frac{j r \pi}{n}, \pi, j\right) \quad \text { and } \quad \tilde{P}_{r}^{\prime}=e_{4}+\sum_{j=1, j \text { odd }}^{n-1} q\left(\frac{j r \pi}{n}, 0, j\right),
$$

and for $r$ odd:

$$
\tilde{P}_{r}=e_{4}+\sum_{j=1, j \text { odd }}^{n-1} q\left(-\frac{j r \pi}{n}, \pi, j\right) \quad \text { and } \quad \tilde{P}_{r}^{\prime}=e_{3}+\sum_{j=1, j \text { odd }}^{n-1} q\left(\frac{j r \pi}{n}, 0, j\right) .
$$

From A.2, we get: $S(q(\alpha, \beta, j))=q(-\alpha, \beta, j)$. 
The calculation of $\Delta\left(Q_{r}\right)$ in the case $n$ even is similar with

$$
\tilde{P}_{r}=\sum_{j=1, j \text { odd }}^{n-1} q\left(-\frac{j r \pi}{n}, \pi, j\right) \quad \text { and } \quad \tilde{P}_{r}^{\prime}=\sum_{j=1, j \text { odd }}^{n-1} q\left(\frac{j r \pi}{n}, 0, j\right),
$$

and with, for $r$ even:

$$
Q_{r}=e_{1}+e_{4}+\sum_{j=1, j \text { even }}^{n-1} q\left(0, \frac{j r \pi}{n}, j\right) \quad \text { and } \quad Q_{r}^{\prime}=e_{2}+e_{3}+\sum_{j=1, j \text { even }}^{n-1} q\left(0, \frac{j r \pi+\pi}{n}, j\right),
$$

and for $r$ odd:

$$
Q_{r}=e_{1}+e_{3}+\sum_{j=1, j \text { even }}^{n-1} q\left(0, \frac{j r \pi}{n}, j\right) \quad \text { and } \quad Q_{r}^{\prime}=e_{2}+e_{4}+\sum_{j=1, j \text { even }}^{n-1} q\left(0, \frac{j r \pi+\pi}{n}, j\right) .
$$

\section{A.5. Coproducts of Jones projections for coideal subalgebras of dimension $2 n$.}

A.5.1. Standard coproduct expressions. We need to calculate the twisted coproduct of certain projections. We start with their standard coproducts in $\mathbb{C}\left[D_{2 n}\right]$, using the results of 2.8.4.

Proposition A.5.1. The standard coproduct $\Delta_{s}$ of the Kac algebra of the dihedral group $D_{2 n}$ satisfies:

$$
\begin{aligned}
\Delta_{s}\left(e_{1}+e_{2}\right)= & \left(e_{1}+e_{2}\right) \otimes\left(e_{1}+e_{2}\right)+\left(e_{3}+e_{4}\right) \otimes\left(e_{3}+e_{4}\right) \\
& +\sum_{j=1}^{n-1} e_{1,1}^{j} \otimes e_{2,2}^{j}+e_{2,2}^{j} \otimes e_{1,1}^{j} \\
\Delta_{s}\left(e_{1}+e_{3}\right)= & \left(e_{1}+e_{3}\right) \otimes\left(e_{1}+e_{3}\right)+\left(e_{2}+e_{4}\right) \otimes\left(e_{2}+e_{4}\right) \\
& +\frac{1}{2} \sum_{j=1}^{n-1}\left(e_{1,1}^{j}+e_{2,2}^{n-j}\right) \otimes\left(e_{2,2}^{j}+e_{1,1}^{n-j}\right)+\left(e_{1,2}^{j}-e_{2,1}^{n-j}\right) \otimes\left(e_{2,1}^{j}-e_{1,2}^{n-j}\right) \\
\Delta_{s}\left(e_{1}+e_{4}\right)= & \left(e_{1}+e_{4}\right) \otimes\left(e_{1}+e_{4}\right)+\left(e_{2}+e_{3}\right) \otimes\left(e_{2}+e_{3}\right) \\
& +\frac{1}{2} \sum_{j=1}^{n-1}\left(e_{1,1}^{n-j}+e_{2,2}^{j}\right) \otimes\left(e_{1,1}^{j}+e_{2,2}^{n-j}\right)+\left(e_{1,2}^{j}+e_{2,1}^{n-j}\right) \otimes\left(e_{2,1}^{j}+e_{1,2}^{n-j}\right) \\
\Delta_{s}\left(e_{3}+e_{4}\right)= & \left(e_{1}+e_{2}\right) \otimes\left(e_{3}+e_{4}\right)+\left(e_{3}+e_{4}\right) \otimes\left(e_{1}+e_{2}\right) \\
& +\sum_{j=1}^{n-1} e_{1,1}^{n-j} \otimes e_{1,1}^{j}+e_{2,2}^{n-j} \otimes e_{2,2}^{j} \\
\Delta_{s}\left(e_{1}+e_{2}+e_{3}+e_{4}\right)= & \left(e_{1}+e_{2}+e_{3}+e_{4}\right) \otimes\left(e_{1}+e_{2}+e_{3}+e_{4}\right) \\
& +\sum_{j=1}^{n-1}\left(e_{1,1}^{n-j}+e_{2,2}^{j}\right) \otimes\left(e_{1,1}^{j}+e_{2,2}^{n-j}\right) \\
& e_{1,1}^{j} \otimes\left(e_{1}+e_{2}\right)+e_{2,2}^{n-j} \otimes\left(e_{3}+e_{4}\right)+\left(e_{1}+e_{2}\right) \otimes e_{1,1}^{j}+\left(e_{3}+e_{4}\right) \otimes e_{2,2}^{n-j} \\
& +\sum_{j^{\prime}<j} e_{1,1}^{j-j^{\prime}} \otimes e_{1,1}^{j^{\prime}}+e_{2,2}^{n-\left(j-j^{\prime}\right)} \otimes e_{2,2}^{n-j^{\prime}} \\
& +\sum_{j^{\prime}>j} e_{2,2}^{j^{\prime}-j} \otimes e_{1,1}^{j^{\prime}}+e_{1,1}^{n-\left(j^{\prime}-j\right)} \otimes e_{2,2}^{n-j^{\prime}} \\
\Delta_{s}\left(e_{1,1}^{j}\right) &
\end{aligned}
$$


Proof. The projections $e_{1}+e_{2}$ and of $e_{1}+e_{2}+e_{3}+e_{4}$ are the Jones projections of the subgroups generated respectively by $\lambda(a)$ and $\lambda\left(a^{2}\right)$. Their standard coproducts can be derived from 2.8.4.

From $e_{1}+e_{4}=\frac{1}{2 n} \sum_{k=0}^{n-1}\left(\lambda\left(a^{2 k}\right)+\lambda\left(b a^{2 k}\right)\right)$, and using the formulas of $\mathrm{A} .2$ we get:

$$
\begin{aligned}
\Delta_{s}\left(e_{1}+e_{4}\right)= & \frac{1}{2 n} \sum_{k=0}^{n-1} \lambda\left(a^{2 k}\right) \otimes \lambda\left(a^{2 k}\right)+\lambda\left(b a^{2 k}\right) \otimes \lambda\left(b a^{2 k}\right) \\
= & \frac{1}{2} \Delta_{s}\left(e_{1}+e_{2}+e_{3}+e_{4}\right) \\
& +\frac{1}{2 n} \sum_{k=0}^{n-1} \lambda\left(b a^{2 k}\right) \otimes\left(\left(e_{1}+e_{4}\right)-\left(e_{2}+e_{3}\right)+\sum_{j=1}^{n-1} \mathrm{e}^{-2 i j k \pi / n} e_{1,2}^{j}+\mathrm{e}^{2 i j k \pi / n} e_{2,1}^{j}\right) \\
= & \frac{1}{2} \Delta_{s}\left(e_{1}+e_{2}+e_{3}+e_{4}\right)+\frac{1}{2}\left(\left(e_{1}+e_{4}\right)-\left(e_{2}+e_{3}\right)\right) \otimes\left(\left(e_{1}+e_{4}\right)-\left(e_{2}+e_{3}\right)\right) \\
& +\sum_{j=1}^{n-1}\left(\frac{1}{2 n} \sum_{k=0}^{n-1} \mathrm{e}^{-2 i j k \pi / n} \lambda\left(b a^{2 k}\right) \otimes e_{1,2}^{j}+\frac{1}{2 n} \sum_{k=0}^{n-1} \mathrm{e}^{2 i j k \pi / n} \lambda\left(b a^{2 k}\right) \otimes e_{2,1}^{j}\right) \\
= & \left(e_{1}+e_{4}\right) \otimes\left(e_{1}+e_{4}\right)+\left(e_{2}+e_{3}\right) \otimes\left(e_{2}+e_{3}\right) \\
& +\frac{1}{2} \sum_{j=1}^{n-1}\left(e_{1,1}^{n-j}+e_{2,2}^{j}\right) \otimes\left(e_{1,1}^{j}+e_{2,2}^{n-j}\right)+\left(e_{2,1}^{j}+e_{1,2}^{n-j}\right) \otimes e_{1,2}^{j}+\left(e_{1,2}^{j}+e_{2,1}^{n-j}\right) \otimes e_{2,1}^{j} \\
= & \left(e_{1}+e_{4}\right) \otimes\left(e_{1}+e_{4}\right)+\left(e_{2}+e_{3}\right) \otimes\left(e_{2}+e_{3}\right) \\
& +\frac{1}{2} \sum_{j=1}^{n-1}\left(e_{1,1}^{n-j}+e_{2,2}^{j}\right) \otimes\left(e_{1,1}^{j}+e_{2,2}^{n-j}\right)+\left(e_{1,2}^{j}+e_{2,1}^{n-j}\right) \otimes\left(e_{2,1}^{j}+e_{1,2}^{n-j}\right)
\end{aligned}
$$

The other coproduct expressions can be calculated similarly.

A.5.2. Twisted coproduct expressions. Using computer exploration as described in B.4.3. and with some factorization efforts, one gets:

For $n=3$ :

$$
\begin{aligned}
\Delta\left(e_{1}+e_{2}\right)= & \left(e_{1}+e_{2}\right) \otimes\left(e_{1}+e_{2}\right)+\left(e_{3}+e_{4}\right) \otimes\left(e_{3}+e_{4}\right) \\
& +r_{1,1}^{1} \otimes r_{1,1}^{1}+r_{2,2}^{1} \otimes r_{2,2}^{1}+e_{1,1}^{2} \otimes e_{2,2}^{2}+e_{2,2}^{2} \otimes e_{1,1}^{2}, \\
\Delta\left(e_{1}+e_{3}\right)= & \left(e_{1}+e_{3}\right) \otimes\left(e_{1}+e_{3}\right)+\left(e_{2}+e_{4}\right) \otimes\left(e_{2}+e_{4}\right) \\
& +\frac{1}{2}\left(\left(e_{2,2}^{1}+r_{1,1}^{2}\right) \otimes\left(e_{1,1}^{1}+r_{1,1}^{2}\right)+\left(e_{1,1}^{1}+r_{2,2}^{2}\right) \otimes\left(e_{2,2}^{1}+r_{2,2}^{2}\right)\right) \\
& +\frac{1}{2}\left(\left(e_{1,2}^{1}+r_{2,1}^{2}\right) \otimes\left(e_{2,1}^{1}+r_{2,1}^{2}\right)+\left(e_{2,1}^{1}+r_{1,2}^{2}\right) \otimes\left(e_{1,2}^{1}+r_{1,2}^{2}\right)\right), \\
\Delta\left(e_{1}+e_{4}\right)= & \left(e_{1}+e_{4}\right) \otimes\left(e_{1}+e_{4}\right)+\left(e_{2}+e_{3}\right) \otimes\left(e_{2}+e_{3}\right) \\
& +\frac{1}{2}\left(\left(e_{1,1}^{1}+r_{1,1}^{2}\right) \otimes\left(e_{2,2}^{1}+r_{1,1}^{2}\right)+\left(e_{2,2}^{1}+r_{2,2}^{2}\right) \otimes\left(e_{1,1}^{1}+r_{2,2}^{2}\right)\right) \\
& +\frac{1}{2}\left(\left(e_{1,2}^{1}-r_{1,2}^{2}\right) \otimes\left(e_{2,1}^{1}-r_{1,2}^{2}\right)+\left(e_{2,1}^{1}-r_{2,1}^{2}\right) \otimes\left(e_{1,2}^{1}-r_{2,1}^{2}\right)\right), \\
\Delta\left(e_{1}+e_{2}+e_{3}\right. & \left.+e_{4}\right)=\left(e_{1}+e_{2}+e_{3}+e_{4}\right) \otimes\left(e_{1}+e_{2}+e_{3}+e_{4}\right) \\
& +\left(r_{1,1}^{1}+e_{2,2}^{2}\right) \otimes\left(r_{1,1}^{1}+e_{1,1}^{2}\right)+\left(r_{2,2}^{1}+e_{1,1}^{2}\right) \otimes\left(r_{2,2}^{1}+e_{2,2}^{2}\right) .
\end{aligned}
$$


For $n=4$ :

$$
\begin{aligned}
\Delta\left(e_{1}+e_{2}\right)= & \left(e_{1}+e_{2}\right) \otimes\left(e_{1}+e_{2}\right)+\left(e_{3}+e_{4}\right) \otimes\left(e_{3}+e_{4}\right)+r_{1,1}^{1} \otimes r_{1,1}^{1} \\
& +r_{2,2}^{1} \otimes r_{2,2}^{1}+e_{1,1}^{2} \otimes e_{2,2}^{2}+e_{2,2}^{2} \otimes e_{1,1}^{2}+r_{1,1}^{3} \otimes r_{1,1}^{3}+r_{2,2}^{3} \otimes r_{2,2}^{3} \\
\Delta\left(e_{1}+e_{3}\right)= & \left(e_{1}+e_{3}\right) \otimes\left(e_{1}+e_{3}\right)+\left(e_{2}+e_{4}\right) \otimes\left(e_{2}+e_{4}\right), \\
& +\frac{1}{2}\left(\left(e_{1,1}^{1}+e_{1,1}^{3}\right) \otimes\left(e_{2,2}^{1}+e_{2,2}^{3}\right)+\left(e_{2,2}^{1}+e_{2,2}^{3}\right) \otimes\left(e_{1,1}^{1}+e_{1,1}^{3}\right)\right) \\
& +\frac{1}{2}\left(\left(e_{1,2}^{2}-e_{1,2}^{3}\right) \otimes\left(e_{2,1}^{1}-e_{2,1}^{3}\right)+\left(e_{2,1}^{1}-e_{2,1}^{3}\right) \otimes\left(e_{1,2}^{1}-e_{1,2}^{3}\right)\right) \\
& +r_{1,1}^{2} \otimes r_{1,1}^{2}+r_{2,2}^{2} \otimes r_{2,2}^{2}, \\
\Delta\left(e_{1}+e_{4}\right)= & \left(e_{1}+e_{4}\right) \otimes\left(e_{1}+e_{4}\right)+\left(e_{2}+e_{3}\right) \otimes\left(e_{2}+e_{3}\right) \\
& +\frac{1}{2}\left(\left(e_{1,1}^{1}+e_{2,2}^{3}\right) \otimes\left(e_{2,2}^{1}+e_{1,1}^{3}\right)+\left(e_{2,2}^{1}+e_{1,1}^{3}\right) \otimes\left(e_{1,1}^{1}+e_{2,2}^{3}\right)\right) \\
& +\frac{1}{2}\left(\left(e_{1,2}^{1}+e_{2,1}^{3}\right) \otimes\left(e_{2,1}^{1}+e_{1,2}^{3}\right)+\left(e_{2,1}^{1}+e_{1,2}^{3}\right) \otimes\left(e_{1,2}^{1}+e_{2,1}^{3}\right)\right) \\
& +p_{1,1}^{2} \otimes p_{1,1}^{2}+p_{2,2}^{2} \otimes p_{2,2}^{2}, \\
\Delta\left(e_{1}+e_{2}+\right. & \left.e_{3}+e_{4}\right)=\left(e_{1}+e_{2}+e_{3}+e_{4}\right) \otimes\left(e_{1}+e_{2}+e_{3}+e_{4}\right) \\
& +\left(r_{1,1}^{1}+r_{2,2}^{3}\right) \otimes\left(r_{1,1}^{1}+r_{2,2}^{3}\right)+\left(r_{2,2}^{1}+r_{1,1}^{3}\right) \otimes\left(r_{2,2}^{1}+r_{1,1}^{3}\right) \\
& +\left(e_{1,1}^{2}+e_{2,2}^{2}\right) \otimes\left(e_{1,1}^{2}+e_{2,2}^{2}\right) .
\end{aligned}
$$

Using the conjugation rules of Remark A.3, the formulas for the standard coproduct, and the expressions of the twisted coproduct for $n=3$ and $n=4$, and with the help of the computer as described in B.4.3, we get:

$$
\begin{aligned}
\Delta\left(e_{1}+e_{2}\right)= & \left(e_{1}+e_{2}\right) \otimes\left(e_{1}+e_{2}\right)+\left(e_{3}+e_{4}\right) \otimes\left(e_{3}+e_{4}\right) \\
& +\sum_{j=1, j \text { odd }}^{n-1} r_{1,1}^{j} \otimes r_{1,1}^{j}+r_{2,2}^{j} \otimes r_{2,2}^{j}+\sum_{j=1, j \text { even }}^{n-1} e_{1,1}^{j} \otimes e_{2,2}^{j}+e_{2,2}^{j} \otimes e_{1,1}^{j} .
\end{aligned}
$$

For $n$ odd:

$$
\begin{aligned}
\Delta\left(e_{1}+e_{3}\right)= & \left(e_{1}+e_{3}\right) \otimes\left(e_{1}+e_{3}\right)+\left(e_{2}+e_{4}\right) \otimes\left(e_{2}+e_{4}\right) \\
& +\frac{1}{2} \sum_{j=1, j \text { odd }}^{n-1}\left(\left(e_{2,2}^{j}+r_{1,1}^{n-j}\right) \otimes\left(e_{1,1}^{j}+r_{1,1}^{n-j}\right)+\left(e_{1,1}^{j}+r_{2,2}^{n-j}\right) \otimes\left(e_{2,2}^{j}+r_{2,2}^{n-j}\right)\right) \\
& +\frac{1}{2} \sum_{j=1, j \text { odd }}^{n-1}\left(\left(e_{1,2}^{j}+r_{2,1}^{n-j}\right) \otimes\left(e_{2,1}^{j}+r_{2,1}^{n-j}\right)+\left(e_{2,1}^{j}+r_{1,2}^{n-j}\right) \otimes\left(e_{1,2}^{j}+r_{1,2}^{n-j}\right)\right), \\
\Delta\left(e_{1}+e_{4}\right)= & \left(e_{1}+e_{4}\right) \otimes\left(e_{1}+e_{4}\right)+\left(e_{2}+e_{3}\right) \otimes\left(e_{2}+e_{3}\right) \\
& +\frac{1}{2} \sum_{j=1, j \text { odd }}^{n-1}\left(\left(e_{1,1}^{j}+r_{1,1}^{n-j}\right) \otimes\left(e_{2,2}^{j}+r_{1,1}^{n-j}\right)+\left(e_{2,2}^{j}+r_{2,2}^{n-j}\right) \otimes\left(e_{1,1}^{j}+r_{2,2}^{n-j}\right)\right) \\
& +\frac{1}{2} \sum_{j=1, j \text { odd }}^{n-1}\left(\left(e_{1,2}^{j}-r_{1,2}^{n-j}\right) \otimes\left(e_{2,1}^{j}-r_{1,2}^{n-j}\right)+\left(e_{2,1}^{j}-r_{2,1}^{n-j}\right) \otimes\left(e_{1,2}^{j}-r_{2,1}^{n-j}\right)\right),
\end{aligned}
$$




$$
\begin{aligned}
\Delta\left(e_{1}+e_{2}+\right. & \left.e_{3}+e_{4}\right)=\left(e_{1}+e_{2}+e_{3}+e_{4}\right) \otimes\left(e_{1}+e_{2}+e_{3}+e_{4}\right) \\
& +\sum_{j=1, j \text { odd }}^{n-1}\left(\left(r_{1,1}^{j}+e_{2,2}^{n-j}\right) \otimes\left(r_{1,1}^{j}+e_{1,1}^{n-j}\right)+\left(r_{2,2}^{j}+e_{1,1}^{n-j}\right) \otimes\left(r_{2,2}^{j}+e_{2,2}^{n-j}\right)\right) \\
\Delta\left(e_{3}+e_{4}\right)= & \left(e_{1}+e_{2}\right) \otimes\left(e_{3}+e_{4}\right)+\left(e_{3}+e_{4}\right) \otimes\left(e_{1}+e_{2}\right) \\
& +\sum_{j=1, j \text { even }}^{n-1} r_{1,1}^{n-j} \otimes e_{1,1}^{j}+r_{2,2}^{n-j} \otimes e_{2,2}^{j}+e_{1,1}^{j} \otimes r_{2,2}^{n-j}+e_{2,2}^{j} \otimes r_{1,1}^{n-j}, \\
\Delta\left(e_{1,1}^{2}\right)= & e_{1,1}^{2} \otimes\left(e_{1}+e_{2}\right)+r_{1,1}^{n-2} \otimes\left(e_{3}+e_{4}\right)+\left(e_{1}+e_{2}\right) \otimes e_{1,1}^{2}+\left(e_{3}+e_{4}\right) \otimes r_{2,2}^{n-2} \\
& +r_{2,2}^{1} \otimes r_{1,1}^{1}+e_{2,2}^{n-1} \otimes e_{2,2}^{n-1} \\
& +\sum_{j>2, j \text { even }}^{n-1} e_{2,2}^{j-2} \otimes e_{1,1}^{j}+r_{2,2}^{n-j+2} \otimes r_{2,2}^{n-j} \\
& +\sum_{j>2, j \text { odd }}^{n-1} r_{1,1}^{j-2} \otimes r_{1,1}^{j}+e_{1,1}^{n-j+2} \otimes e_{2,2}^{n-j} .
\end{aligned}
$$

For $n$ even:

$$
\begin{aligned}
\Delta\left(e_{1}+e_{3}\right)= & \left(e_{1}+e_{3}\right) \otimes\left(e_{1}+e_{3}\right)+\left(e_{2}+e_{4}\right) \otimes\left(e_{2}+e_{4}\right) \\
& +\frac{1}{2} \sum_{j=1, j \text { odd }}^{n-1}\left(\left(r_{2,2}^{j}+r_{1,1}^{n-j}\right) \otimes\left(r_{2,2}^{j}+r_{1,1}^{n-j}\right)+\left(r_{2,1}^{j}-r_{1,2}^{n-j}\right) \otimes\left(r_{2,1}^{j}-r_{1,2}^{n-j}\right)\right) \\
& +\frac{1}{2} \sum_{j=1, j \text { even }}^{n-1}\left(\left(e_{1,1}^{j}+e_{2,2}^{n-j}\right) \otimes\left(e_{2,2}^{j}+e_{1,1}^{n-j}\right)+\left(e_{1,2}^{j}-e_{2,1}^{n-j}\right) \otimes\left(e_{2,1}^{j}-e_{1,2}^{n-j}\right)\right), \\
\Delta\left(e_{1}+e_{4}\right)= & \left(e_{1}+e_{4}\right) \otimes\left(e_{1}+e_{4}\right)+\left(e_{2}+e_{3}\right) \otimes\left(e_{2}+e_{3}\right) \\
& +\frac{1}{2} \sum_{j=1, j \text { odd }}^{n-1}\left(\left(r_{1,1}^{j}+r_{2,2}^{n-j}\right) \otimes\left(r_{1,1}^{j}+r_{2,2}^{n-j}\right)+\left(r_{2,1}^{j}+r_{1,2}^{n-j}\right) \otimes\left(r_{2,1}^{j}+r_{1,2}^{n-j}\right)\right) \\
& +\frac{1}{2} \sum_{j=1, j \text { even }}^{n-1}\left(\left(e_{1,1}^{n-j}+e_{2,2}^{j}\right) \otimes\left(e_{1,1}^{j}+e_{2,2}^{n-j}\right)+\left(e_{1,2}^{j}+e_{2,1}^{n-j}\right) \otimes\left(e_{2,1}^{j}+e_{1,2}^{n-j}\right)\right), \\
\Delta\left(e_{3}+e_{4}\right)= & \left(e_{1}+e_{2}\right) \otimes\left(e_{3}+e_{4}\right)+\left(e_{3}+e_{4}\right) \otimes\left(e_{1}+e_{2}\right) \\
& +\sum_{j=1, j \text { odd }}^{n-1} r_{1,1}^{j} \otimes r_{2,2}^{n-j}+r_{2,2}^{j} \otimes r_{1,1}^{n-j} \\
& +\sum_{j=1, j \text { even }}^{n-1} e_{1,1}^{n-j} \otimes e_{1,1}^{j}+e_{2,2}^{n-j} \otimes e_{2,2}^{j} \\
& +\sum_{j=1, j \text { even }}^{n-1}\left(e_{1,1}^{j}+e_{2,2}^{n-j}\right) \otimes\left(e_{2,2}^{j}+e_{1,1}^{n-j}\right), \\
& +\sum_{j=1, j \text { odd }}^{n-1}\left(r_{1,1}^{j}+r_{2,2}^{n-j}\right) \otimes\left(r_{1,1}^{j}+r_{2,2}^{n-j}\right) \\
\Delta\left(e_{1}+e_{2}+\right. & \left.e_{3}+e_{4}\right)=\left(e_{2}+e_{3}+e_{4}\right) \otimes\left(e_{1}+e_{2}+e_{3}+e_{4}\right) \\
& \left.\sum_{j}^{n-1}\right)
\end{aligned}
$$




$$
\begin{aligned}
\Delta\left(e_{1,1}^{2}\right)= & e_{1,1}^{2} \otimes\left(e_{1}+e_{2}\right)+e_{2,2}^{n-2} \otimes\left(e_{3}+e_{4}\right)+\left(e_{1}+e_{2}\right) \otimes e_{1,1}^{2}+\left(e_{3}+e_{4}\right) \otimes e_{2,2}^{n-2} \\
& +r_{2,2}^{1} \otimes r_{1,1}^{1}+r_{1,1}^{n-1} \otimes r_{2,2}^{n-1} \\
& +\sum_{j>2, j \text { even }}^{n-1} e_{2,2}^{j-2} \otimes e_{1,1}^{j}+e_{1,1}^{n-j+2} \otimes e_{2,2}^{n-j} \\
& +\sum_{j>2, j \text { odd }}^{n-1} r_{1,1}^{j-2} \otimes r_{1,1}^{j}+r_{2,2}^{n-j+2} \otimes r_{2,2}^{n-j} .
\end{aligned}
$$

A.6. Coproducts of $v$ and $w$ of $K_{3}$ in $K D(2 m)$. In this section, we calculate the coproducts of the unitary elements $v$ and $w$ of $K_{3}$ in $K D(2 m)$ needed in 9.1.1.

Proposition A.6. With the notations of 9.1.1, the unitary elements $v$ and $w$ of $K_{3}$ expand as follow in the group basis:

$$
\begin{aligned}
v & =\lambda(b a) B_{0}-\frac{1}{2} B_{1}\left[\mathrm{i}\left(\lambda(a)-\lambda\left(a^{-1}\right)\right)+\lambda(b a)+\lambda\left(b a^{-1}\right)\right] \\
w & =\lambda\left(b a^{-1}\right) B_{0}+\frac{1}{2} B_{1}\left[\mathrm{i}\left(\lambda(a)-\lambda\left(a^{-1}\right)\right)-\lambda(b a)-\lambda\left(b a^{-1}\right)\right]
\end{aligned}
$$

The coproduct of $v$ satisfies : $\Delta(v)=v \otimes B_{0} v+w \otimes B_{1} v$.

Furthermore: $\Delta(w)=w \otimes B_{0} w+v \otimes B_{1} w$.

Proof. A straightforward calculation gives the expressions in the group basis.

We now check the expression of $\Delta(v)$ by proving that untwisting it yields back the standard coproduct of $v$. Namely, setting $D_{i, j}=\left(B_{i} \otimes B_{j}\right)\left(v \otimes B_{0} v+w \otimes B_{1} v\right)$ for $i=1,2$ and $j=1,2$, we check the equalities: $\Omega^{*} D_{i, j} \Omega=\left(B_{i} \otimes B_{j}\right) \Delta_{s}(v)$, for $i=1,2$ and $j=1,2$.

Note first that $B_{1}$ is the projection on odd factors and that

$$
\Delta\left(B_{0}\right)=B_{0} \otimes B_{0}+B_{1} \otimes B_{1} \quad \text { et } \quad \Delta\left(B_{1}\right)=B_{0} \otimes B_{1}+B_{1} \otimes B_{0}
$$

since $\left.\lambda\left(a^{n}\right)\right)$ belongs to the intrinsic group. Using Remark A.3, it follows that:

$$
\Delta_{s}\left(v B_{0}\right)=\lambda(b a) B_{0} \otimes \lambda(b a) B_{0}+\lambda(b a) B_{1} \otimes \lambda(b a) B_{1}=\Omega^{*} D_{0,0} \Omega+\Omega^{*} D_{1,1} \Omega
$$

The action of $\Omega$ on $x \otimes y$ depends only on the parity of the factors containing $x$ and $y$, and in particular is independent of $n$ (even). It is therefore sufficient to use the following formulas which have been obtained by computer in $K D(4)$.

- For tensors in $K D(2 m)^{2 j+1} \otimes K D(2 m)^{2 k}$ :

$$
\begin{aligned}
\Omega^{*}\left(r_{1,2} \otimes e_{1,2}\right) \Omega & =\frac{\mathrm{i}}{2}\left(e_{1,1} \otimes e_{1,1}-e_{2,2} \otimes e_{2,2}\right)-\frac{1}{2}\left(e_{1,2} \otimes e_{1,2}+e_{2,1} \otimes e_{2,1}\right) \\
\Omega^{*}\left(r_{1,2} \otimes e_{2,1}\right) \Omega & =\frac{\mathrm{i}}{2}\left(e_{1,1} \otimes e_{2,2}-e_{2,2} \otimes e_{1,1}\right)-\frac{1}{2}\left(e_{1,2} \otimes e_{2,1}+e_{2,1} \otimes e_{1,2}\right) \\
\Omega^{*}\left(r_{2,1} \otimes e_{1,2}\right) \Omega & =\frac{\mathrm{i}}{2}\left(e_{2,2} \otimes e_{1,1}-e_{1,1} \otimes e_{2,2}\right)-\frac{1}{2}\left(e_{2,1} \otimes e_{1,2}+e_{1,2} \otimes e_{2,1}\right) \\
\Omega^{*}\left(r_{2,1} \otimes e_{2,1}\right) \Omega & =\frac{\mathrm{i}}{2}\left(e_{2,2} \otimes e_{2,2}-e_{1,1} \otimes e_{1,1}\right)-\frac{1}{2}\left(e_{1,2} \otimes e_{1,2}+e_{2,1} \otimes e_{2,1}\right)
\end{aligned}
$$


- For tensors in $K D(2 m)^{2 j+1} \otimes\left(\mathbb{C} e_{1} \oplus \mathbb{C} e_{2} \oplus \mathbb{C} e_{3} \oplus \mathbb{C} e_{4}\right)$ :

$$
\begin{aligned}
\Omega^{*}\left[r_{1,2} \otimes\left(\left(e_{1}+e_{3}\right)-\left(e_{2}+e_{4}\right)\right)\right] \Omega & =\frac{\mathrm{i}}{2}\left[\left(e_{1,1}-e_{2,2}\right) \otimes\left(\left(e_{1}+e_{2}\right)-\left(e_{3}+e_{4}\right)\right)\right] \\
& -\frac{1}{2}\left[\left(e_{1,2}+e_{2,1}\right) \otimes\left(\left(e_{1}+e_{3}\right)-\left(e_{2}+e_{4}\right)\right)\right] \\
\Omega^{*}\left[r_{2,1} \otimes\left(\left(e_{1}+e_{3}\right)-\left(e_{2}+e_{4}\right)\right)\right] \Omega & =\frac{\mathrm{i}}{2}\left[\left(e_{2,2}-e_{1,1}\right) \otimes\left(\left(e_{1}+e_{2}\right)-\left(e_{3}+e_{4}\right)\right)\right] \\
& -\frac{1}{2}\left[\left(e_{1,2}+e_{2,1}\right) \otimes\left(\left(e_{1}+e_{3}\right)-\left(e_{2}+e_{4}\right)\right)\right]
\end{aligned}
$$

- For tensors in $K D(2 m)^{2 k} \otimes K D(2 m)^{2 j+1}$ :

$$
\begin{aligned}
& \Omega^{*}\left(e_{1,2} \otimes r_{1,2}\right) \Omega=\frac{\mathrm{i}}{2}\left(e_{2,2} \otimes e_{1,1}-e_{1,1} \otimes e_{2,2}\right)-\frac{1}{2}\left(e_{1,2} \otimes e_{2,1}+e_{2,1} \otimes e_{1,2}\right) \\
& \Omega^{*}\left(e_{2,1} \otimes r_{1,2}\right) \Omega=\frac{\mathrm{i}}{2}\left(e_{1,1} \otimes e_{1,1}-e_{2,2} \otimes e_{2,2}\right)-\frac{1}{2}\left(e_{1,2} \otimes e_{1,2}+e_{2,1} \otimes e_{2,1}\right) \\
& \Omega^{*}\left(e_{1,2} \otimes r_{2,1}\right) \Omega=\frac{\mathrm{i}}{2}\left(e_{2,2} \otimes e_{2,2}-e_{1,1} \otimes e_{1,1}\right)-\frac{1}{2}\left(e_{1,2} \otimes e_{1,2}+e_{2,1} \otimes e_{2,1}\right) \\
& \Omega^{*}\left(e_{2,1} \otimes r_{2,1}\right) \Omega=\frac{\mathrm{i}}{2}\left(e_{1,1} \otimes e_{2,2}-e_{2,2} \otimes e_{1,1}\right)-\frac{1}{2}\left(e_{2,1} \otimes e_{1,2}+e_{1,2} \otimes e_{2,1}\right)
\end{aligned}
$$

- For tensors in $\left(\mathbb{C} e_{1} \oplus \mathbb{C} e_{2} \oplus \mathbb{C} e_{3} \oplus \mathbb{C} e_{4}\right) \otimes K D(2 m)^{2 j+1}$ :

$$
\begin{aligned}
\Omega^{*}\left[\left(\left(e_{1}+e_{3}\right)-\left(e_{2}+e_{4}\right)\right) \otimes r_{1,2}\right] \Omega & =\frac{\mathrm{i}}{2}\left[\left(\left(e_{1}+e_{2}\right)-\left(e_{3}+e_{4}\right)\right) \otimes\left(e_{1,1}-e_{2,2}\right)\right] \\
& -\frac{1}{2}\left[\left(\left(e_{1}+e_{3}\right)-\left(e_{2}+e_{4}\right)\right) \otimes\left(e_{1,2}+e_{2,1}\right)\right] \\
\Omega^{*}\left[\left(\left(e_{1}+e_{3}\right)-\left(e_{2}+e_{4}\right)\right) \otimes r_{2,1}\right] \Omega & =\frac{\mathrm{i}}{2}\left[\left(\left(e_{1}+e_{2}\right)-\left(e_{3}+e_{4}\right)\right) \otimes\left(e_{2,2}-e_{1,1}\right)\right] \\
& -\frac{1}{2}\left[\left(\left(e_{1}+e_{3}\right)-\left(e_{2}+e_{4}\right)\right) \otimes\left(e_{1,2}+e_{2,1}\right)\right]
\end{aligned}
$$

It follows that $\Delta_{s}\left(v B_{1}\right)=\Omega^{*} D_{0,1} \Omega+\Omega^{*} D_{1,0} \Omega$. Let us detail, for example, the calculation of $\Omega^{*} D_{1,0} \Omega$ :

$$
\begin{aligned}
\Omega^{*}\left(B_{1} v \otimes B_{0} v\right) \Omega & =\sum_{j=0}^{m-1} \Omega^{*}\left(\left(\epsilon^{-(2 j+1)} r_{1,2}^{2 j+1}+\epsilon^{2 j+1} r_{2,1}^{2 j+1}\right) \otimes\left(\left(e_{1}+e_{3}\right)-\left(e_{2}+e_{4}\right)\right)\right) \Omega \\
& +\sum_{j=0}^{m-1} \sum_{k=1}^{m-1} \Omega^{*}\left(\left(\epsilon^{-(2 j+1)} r_{1,2}^{2 j+1}+\epsilon^{2 j+1} r_{2,1}^{2 j+1}\right) \otimes\left(\epsilon^{-2 k} e_{1,2}^{2 k}+\epsilon^{2 k} e_{2,1}^{2 k}\right)\right) \Omega
\end{aligned}
$$




$$
\begin{aligned}
& =-\frac{\mathrm{i}}{2} \sum_{j=0}^{m-1}\left(\left(\epsilon^{2 j+1} e_{1,1}^{2 j+1}+\epsilon^{-(2 j+1)} e_{2,2}^{2 j+1}\right)-\left(\epsilon^{-(2 j+1)} e_{1,1}^{2 j+1}+\epsilon^{2 j+1} e_{2,2}^{2 j+1}\right)\right) \otimes\left(\left(e_{1}+e_{2}\right)-\left(e_{3}+e_{4}\right)\right) \\
& -\frac{1}{2} \sum_{j=0}^{m-1}\left(\left(\epsilon^{-(2 j+1)} e_{1,2}^{2 j+1}+\epsilon^{2 j+1} e_{2,1}^{2 j+1}\right)+\left(\epsilon^{2 j+1} e_{1,2}^{2 j+1}+\epsilon^{-(2 j+1)} e_{2,1}^{2 j+1}\right)\right) \otimes\left(\left(e_{1}+e_{3}\right)-\left(e_{2}+e_{4}\right)\right) \\
& +\frac{\mathrm{i}}{2} \sum_{j=0}^{m-1} \sum_{k=1}^{m-1} \epsilon^{-(2 j+1)} e_{1,1}^{2 j+1} \otimes \epsilon^{-2 k} e_{1,1}^{2 k}+\epsilon^{-(2 j+1)} e_{1,1}^{2 j+1} \otimes \epsilon^{2 k} e_{2,2}^{2 k} \\
& +\epsilon^{2 j+1} e_{2,2}^{2 j+1} \otimes \epsilon^{-2 k} e_{1,1}^{2 k}+\epsilon^{2 j+1} e_{2,2}^{2 j+1} \otimes \epsilon^{2 k} e_{2,2}^{2 k} \\
& -\epsilon^{-(2 j+1)} e_{2,2}^{2 j+1} \otimes \epsilon^{-2 k} e_{2,2}^{2 k}-\epsilon^{-(2 j+1)} e_{2,2}^{2 j+1} \otimes \epsilon^{2 k} e_{1,1}^{2 k} \\
& -\epsilon^{2 j+1} e_{1,1}^{2 j+1} \otimes \epsilon^{-2 k} e_{2,2}^{2 k}-\epsilon^{2 j+1} e_{1,1}^{2 j+1} \otimes \epsilon^{2 k} e_{1,1}^{2 k} \\
& -\frac{1}{2} \sum_{j=0}^{m-1} \sum_{k=1}^{m-1} \epsilon^{-(2 j+1)} e_{1,2}^{2 j+1} \otimes \epsilon^{-2 k} e_{1,2}^{2 k}+\epsilon^{-(2 j+1)} e_{2,1}^{2 j+1} \otimes \epsilon^{-2 k} e_{2,1}^{2 k} \\
& +\epsilon^{-(2 j+1)} e_{1,2}^{2 j+1} \otimes \epsilon^{2 k} e_{2,1}^{2 k}+\epsilon^{-(2 j+1)} e_{2,1}^{2 j+1} \otimes \epsilon^{2 k} e_{1,2}^{2 k} \\
& +\epsilon^{2 j+1} e_{2,1}^{2 j+1} \otimes \epsilon^{-2 k} e_{1,2}^{2 k}+\epsilon^{2 j+1} e_{1,2}^{2 j+1} \otimes \epsilon^{-2 k} e_{2,1}^{2 k} \\
& +\epsilon^{2 j+1} e_{1,2}^{2 j+1} \otimes \epsilon^{2 k} e_{1,2}^{2 k}+\epsilon^{2 j+1} e_{2,1}^{2 j+1} \otimes \epsilon^{2 k} e_{2,1}^{2 k} \\
& \left.=-\frac{\mathrm{i}}{2} \sum_{j=0}^{m-1}\left(\epsilon^{2 j+1} e_{1,1}^{2 j+1}+\epsilon^{-(2 j+1)} e_{2,2}^{2 j+1}\right) \otimes\left(\left(e_{1}+e_{2}\right)-\left(e_{3}+e_{4}\right)\right)+\sum_{k=1}^{m-1} \epsilon^{2 k} e_{1,1}^{2 k}+\epsilon^{-2 k} e_{2,2}^{2 k}\right) \\
& \left.+\frac{\mathrm{i}}{2} \sum_{j=0}^{m-1}\left(\epsilon^{-(2 j+1)} e_{1,1}^{2 j+1}+\epsilon^{2 j+1} e_{2,2}^{2 j+1}\right) \otimes\left(\left(e_{1}+e_{2}\right)-\left(e_{3}+e_{4}\right)\right)+\sum_{k=1}^{m-1} \epsilon^{-2 k} e_{1,1}^{2 k}+\epsilon^{2 k} e_{2,2}^{2 k}\right) \\
& -\frac{1}{2} \sum_{j=0}^{m-1}\left(\epsilon^{-(2 j+1)} e_{1,2}^{2 j+1}+\epsilon^{2 j+1} e_{2,1}^{2 j+1}\right) \otimes\left(\left(e_{1}+e_{3}\right)-\left(e_{2}+e_{4}\right)+\sum_{k=1}^{m-1} \epsilon^{-2 k} e_{1,2}^{2 k}+\epsilon^{2 k} e_{2,1}^{2 k}\right) \\
& -\frac{1}{2} \sum_{j=0}^{m-1}\left(\epsilon^{2 j+1} e_{1,2}^{2 j+1}+\epsilon^{-(2 j+1)} e_{2,1}^{2 j+1}\right) \otimes\left(\left(e_{1}+e_{3}\right)-\left(e_{2}+e_{4}\right)+\sum_{k=1}^{m-1} \epsilon^{2 k} e_{1,2}^{2 k}+\epsilon^{-2 k} e_{2,1}^{2 k}\right) \\
& =-\frac{\mathrm{i}}{2}\left(\lambda(a) B_{1} \otimes \lambda(a) B_{0}-\lambda\left(a^{-1}\right) B_{1} \otimes \lambda\left(a^{-1}\right) B_{0}\right) \\
& -\frac{1}{2}\left(\lambda(b a) B_{1} \otimes \lambda(b a) B_{0}+\lambda\left(b a^{-1}\right) B_{1} \otimes \lambda\left(b a^{-1}\right) B_{0}\right) .
\end{aligned}
$$

The calculation of the coproduct of $w$ is analogous. 
A.7. Structure of $K_{33}$ in $K_{3}$ of $K D\left(4 m^{\prime}\right)$. The Jones projection of the coideal subalgebra $K_{33}$ of $K_{3}$ in $K D\left(4 m^{\prime}\right)$ is $p_{33}=e_{1}+e_{3}+r_{1,1}^{m}$. Furthermore,

$$
\begin{aligned}
p_{33} & =e_{1}+e_{3}+r_{1,1}^{m} \\
& =\frac{1}{2 n} \sum_{k=0}^{n-1} \lambda\left(a^{2 k}\right)+\lambda\left(b a^{2 k+1}\right)+\frac{1}{2 n} \sum_{k=0}^{n-1}(-1)^{k} \lambda\left(a^{2 k}\right)+\frac{1}{2 n} \sum_{k=0}^{n-1}(-1)^{k} \lambda\left(b a^{2 k+1}\right) \\
& =\frac{1}{n} \sum_{k=0}^{m-1} \lambda\left(a^{4 k}\right)+\lambda\left(b a^{4 k+1}\right)
\end{aligned}
$$

Therefore $p_{33}$ is the Jones projection of the algebra $A$ of the subgroup $D_{2 m^{\prime}}=\left\langle a^{4}, b a\right\rangle$ in $\mathbb{C}\left[D_{2 n}\right]$. Using e.g. Proposition 2.8.2, its standard coproduct can be expressed in terms of the matrix units of $A$. This subalgebra admits four central projections, associated to the factors of dimension 1: $\tilde{e}_{1}=p_{33}, \tilde{e}_{2}, \tilde{e}_{3}$, and $\tilde{e}_{4}$, with

$$
\begin{aligned}
& \tilde{e}_{2}=\frac{1}{2 m} \sum_{k=0}^{m-1}\left(\lambda\left(a^{4 k}\right)-\lambda\left(b a^{4 k+1}\right)\right) \\
& =e_{2}+e_{4}+\frac{1}{2 m} \sum_{j=1}^{n-1} \sum_{k=0}^{m-1}\left(\epsilon_{m}^{2 j k} e_{1,1}^{j}+\epsilon_{m}^{-2 j k} e_{2,2}^{j}\right)-\left(\epsilon_{m}^{-2 j k} \epsilon_{n}^{-j} e_{1,2}^{j}+\epsilon_{m}^{2 j k} \epsilon_{n}^{j} e_{2,1}^{j}\right) \\
& =e_{2}+e_{4}+r_{2,2}^{m} \\
& \tilde{e}_{3}=\frac{1}{2 m} \sum_{k=0}^{m-1}(-1)^{k}\left(\lambda\left(a^{4 k}\right)-\lambda\left(b a^{4 k+1}\right)\right) \\
& =\frac{1}{2 m} \sum_{j=1}^{n-1} \sum_{k=0}^{m-1}(-1)^{k}\left(\epsilon_{m}^{2 j k} e_{1,1}^{j}+\epsilon_{m}^{-2 j k} e_{2,2}^{j}\right)-(-1)^{k}\left(\epsilon_{m}^{-2 j k} \epsilon_{n}^{-j} e_{1,2}^{j}+\epsilon_{m}^{2 j k} \epsilon_{n}^{j} e_{2,1}^{j}\right) \\
& =\frac{1}{2 m} \sum_{j=1}^{n-1} \sum_{k=0}^{m-1}\left(\epsilon_{m}^{2\left(m^{\prime}+j\right) k} e_{1,1}^{j}+\epsilon_{m}^{-2\left(m^{\prime}+j\right) k} e_{2,2}^{j}\right)-\left(\epsilon_{m}^{-2\left(m^{\prime}+j\right) k} \epsilon_{n}^{-j} e_{1,2}^{j}+\epsilon_{m}^{2\left(m^{\prime}+j\right) k} \epsilon_{n}^{j} e_{2,1}^{j}\right) \\
& =\frac{1}{2}\left(e_{1,1}^{m^{\prime}}+e_{2,2}^{m^{\prime}}+\mathrm{e}^{3 i \pi / 4} e_{1,2}^{m^{\prime}}+\mathrm{e}^{-3 i \pi / 4} e_{2,1}^{m^{\prime}}\right)+\frac{1}{2}\left(e_{1,1}^{3 m^{\prime}}+e_{2,2}^{3 m^{\prime}}+\mathrm{e}^{i \pi / 4} e_{1,2}^{3 m^{\prime}}+\mathrm{e}^{-i \pi / 4} e_{2,1}^{3 m^{\prime}}\right) \\
& =q\left(0,-3 \pi / 4, m^{\prime}\right)+q\left(0,-\pi / 4,3 m^{\prime}\right) \\
& \tilde{e}_{4}=\frac{1}{2 m} \sum_{k=0}^{m-1}(-1)^{k}\left(\lambda\left(a^{k}\right)+\lambda\left(b a^{4 k+1}\right)\right) \\
& =\frac{1}{2 m} \sum_{j=1}^{n-1} \sum_{k=0}^{m-1}(-1)^{k}\left(\epsilon_{m}^{2 j k} e_{1,1}^{j}+\epsilon_{m}^{-2 j k} e_{2,2}^{j}\right)+(-1)^{k}\left(\epsilon_{m}^{-2 j k} \epsilon_{n}^{-j} e_{1,2}^{j}+\epsilon_{m}^{2 j k} \epsilon_{n}^{j} e_{2,1}^{j}\right) \\
& =\frac{1}{2 m} \sum_{j=1}^{n-1} \sum_{k=0}^{m-1}\left(\epsilon_{m}^{2\left(m^{\prime}+j\right) k} e_{1,1}^{j}+\epsilon_{m}^{-2\left(m^{\prime}+j\right) k} e_{2,2}^{j}\right)+\left(\epsilon_{m}^{-2\left(m^{\prime}+j\right) k} \epsilon_{n}^{-j} e_{1,2}^{j}+\epsilon_{m}^{2\left(m^{\prime}+j\right) k} \epsilon_{n}^{j} e_{2,1}^{j}\right) \\
& =\frac{1}{2}\left(e_{1,1}^{m^{\prime}}+e_{2,2}^{m^{\prime}}+\mathrm{e}^{-i \pi / 4} e_{1,2}^{m^{\prime}}+\mathrm{e}^{i \pi / 4} e_{2,1}^{m^{\prime}}\right)+\frac{1}{2}\left(e_{1,1}^{3 m^{\prime}}+e_{2,2}^{3 m^{\prime}}+\mathrm{e}^{-3 i \pi / 4} e_{1,2}^{3 m^{\prime}}+\mathrm{e}^{3 i \pi / 4} e_{2,1}^{3 m^{\prime}}\right) \\
& =q\left(0, \pi / 4, m^{\prime}\right)+q\left(0,3 \pi / 4,3 m^{\prime}\right)
\end{aligned}
$$


as well as $m^{\prime}-1$ blocks of dimension 2 whose matrix units are derived from the partial isometries $\tilde{e}_{1,2}^{h}$ with $h=1, \ldots, m^{\prime}-1$ :

$$
\begin{aligned}
\tilde{e}_{1,2}^{h} & =\frac{1}{m} \sum_{k=0}^{m-1} \mathrm{e}^{i h k \pi / m^{\prime}} \lambda\left(b a^{4 k+1}\right) \\
& =\frac{1}{m} \sum_{j=1}^{n-1} \sum_{k=0}^{m-1} \epsilon_{m}^{2 h k}\left(\epsilon_{m}^{-2 j k} \epsilon_{n}^{-j} e_{1,2}^{j}+\epsilon_{m}^{2 j k} \epsilon_{n}^{j} e_{2,1}^{j}\right) \\
& =\frac{1}{m} \sum_{j=1}^{n-1} \sum_{k=0}^{m-1}\left(\epsilon_{m}^{2(h-j) k} \epsilon_{n}^{-j} e_{1,2}^{j}+\epsilon_{m}^{2(h+j) k} \epsilon_{n}^{j} e_{2,1}^{j}\right) \\
& =\epsilon_{n}^{-h} e_{1,2}^{h}+\epsilon_{n}^{-(m+h)} e_{1,2}^{m+h}+\epsilon_{n}^{(m-h)} e_{2,1}^{m-h}+\epsilon_{n}^{(n-h)} e_{2,1}^{n-h} \\
& =\epsilon_{n}^{-h}\left(e_{1,2}^{h}-e_{2,1}^{n-h}\right)+\epsilon_{n}^{-(m+h)}\left(e_{1,2}^{m+h}-e_{2,1}^{n-(m+h)}\right) \\
& =\epsilon_{n}^{-h}\left(\left(e_{1,2}^{h}-e_{2,1}^{n-h}\right)-i\left(e_{1,2}^{m+h}-e_{2,1}^{n-(m+h)}\right)\right) .
\end{aligned}
$$

Upon deformation by $\Omega$, the right legs $\tilde{e}_{1}, \tilde{e}_{2}, \tilde{e}_{3}, \tilde{e}_{4}$, and $\tilde{e}_{i, j}^{h}$ for $h$ even of $\Delta_{s}\left(p_{33}\right)$ are left unchanged; the remaining rights legs $\tilde{e}_{i, j}^{h}$ for $h$ odd become $\epsilon_{n}^{-h}\left[\left(r_{1,2}^{h}-r_{2,1}^{n-h}\right)-i\left(r_{1,2}^{m+h}-\right.\right.$ $\left.\left.r_{2,1}^{n-(m+h)}\right)\right]$. Therefore, $K_{33}$ admits exactly four blocs of dimension 1 , as is the case for $A$.

A.8. Calculations for Theorem 6.1. We complete the demonstration of the self-duality of $K D(n)$ for $n$ odd (Theorem 6.1) by calculating explicitly $\psi(\lambda(a))$ and $\psi(\lambda(b))$ and checking that their coproducts are preserved by $\psi$. The calculations are straightforward albeit lengthy; regrettably, we could not delegate them to the computer in the general case.

A.8.1. Preservation of the coproduct of $\lambda(b)$.

Lemma A.8.1.

$$
\psi(\lambda(b))=4 n \widehat{e_{4}} \quad \text { and } \quad \Delta(\psi(\lambda(b)))=(\psi \otimes \psi)(\Delta(\lambda(b)) .
$$

Proof. From A.1 and [5.2, one can write:

$$
\begin{aligned}
\lambda(b) & =e_{1}-e_{2}-e_{3}+e_{4}+\sum_{j=1}^{n-1} e_{1,2}^{j}+e_{2,1}^{j} \\
& =e_{1}-e_{2}-e_{3}+e_{4}+\sum_{k=1, k \text { odd }}^{n-1}-\left(r_{1,2}^{k}+r_{2,1}^{k}\right)+e_{1,2}^{n-k}+e_{2,1}^{n-k} .
\end{aligned}
$$

It follows that

$$
\begin{aligned}
\psi(\lambda(b))= & \chi_{1}-\chi_{a^{n}}-\chi_{b a^{n}}+\chi_{b}+\sum_{k=1, k \text { odd }}^{n-1}-\left(E_{1,2}^{k}+E_{2,1}^{k}\right)+E_{1,2}^{n-k}+E_{2,1}^{n-k} \\
= & \chi_{1}-\chi_{a^{n}}-\chi_{b a^{n}}+\chi_{b} \\
& +\sum_{k=1, k \text { odd }}^{n-1} \chi_{b a^{n-k}}+\chi_{b a^{n+k}}-\chi_{b a^{k}}-\chi_{b a^{2 n-k}}-\chi_{a^{k}}-\chi_{a^{2 n-k}}+\chi_{a^{n+k}}+\chi_{a^{n-k}} \\
= & \sum_{j=0}^{2 n-1}(-1)^{j}\left(\chi_{a^{j}}+\chi_{b a^{j}}\right)=4 n \widehat{e_{4}} .
\end{aligned}
$$


Then,

$$
\begin{aligned}
& (\psi \otimes \psi)\left(\Delta(\lambda(b))=16 n^{2} \widehat{e_{4}} \otimes \widehat{e_{4}}\right. \\
= & \sum_{j=0}^{2 n-1} \sum_{k=0}^{2 n-1}(-1)^{k+j}\left(\chi_{a^{j}}+\chi_{b a^{j}}\right) \otimes\left(\chi_{a^{k}}+\chi_{b a^{k}}\right) \\
= & \sum_{j=0}^{2 n-1} \sum_{k=0}^{2 n-1}(-1)^{k+j}\left(\chi_{a^{j}} \otimes\left(\chi_{a^{-j} a^{j+k}}+\chi_{a^{-j} b a^{k-j}}\right)+\chi_{b a^{j}} \otimes\left(\chi_{\left(a^{-j} b\right) b a^{k+j}}+\chi_{\left(a^{-j} b\right) a^{k-j}}\right)\right) ;
\end{aligned}
$$

and since $j+k$ and $j-k$ have the same parity:

$$
(\psi \otimes \psi)\left(\Delta(\lambda(b))=\sum_{j=0}^{2 n-1} \sum_{s \in D_{2 n}}(-1)^{j} \chi_{s} \otimes\left(\chi_{s^{-1} a^{j}}+\chi_{s^{-1} b a^{j}}\right)=\Delta\left(4 n \widehat{e_{4}}\right)=\Delta(\psi(\lambda(b))) .\right.
$$

A.8.2. Preservation of the coproduct of $\lambda(a)$.

\section{Lemma A.8.2.}

$\left.\psi(\lambda(a))=n\left(2 \widehat{e_{1,1}^{n-1}}+\widehat{e_{2,2}^{1}}-\widehat{\left(e_{1,1}^{1}\right.}+\widehat{e_{1,2}^{n-1}}+\widehat{e_{2,1}^{n-1}}\right)\right) \quad$ and $\quad \Delta(\psi(\lambda(a)))=(\psi \otimes \psi)(\Delta(\lambda(a))$.

We prove this lemma in the following subsections. In order to split the calculations, using A.1 and $\epsilon=\mathrm{e}^{\mathrm{i} \pi / n}$, we write: $\lambda(a)=U+V$, with

$U=e_{1}+e_{2}+\sum_{k=1, k \text { even }}^{n-1} \epsilon^{k} e_{1,1}^{k}+\epsilon^{-k} e_{2,2}^{k} \quad$ and $\quad V=-e_{3}-e_{4}+\sum_{k=1, k \text { odd }}^{n-1} \epsilon^{k} e_{1,1}^{k}+\epsilon^{-k} e_{2,2}^{k}$.

Hence, since all the terms of $U-\left(e_{1}+e_{2}\right)$ (resp. $\left.V+\left(e_{3}+e_{4}\right)\right)$ are in $M_{2}(\mathbb{C})$ factors of same parity, we may use Remark A.3 to calculate the coproducts of $U$ and $V$.

A.8.3. Expression of $\psi(U)$ and $\psi(V)$.

Lemma A.8.3.

$$
\psi(U)=2 n \widehat{e_{1,1}^{n-1}}, \quad \psi(V)=n\left(\widehat{e_{2,2}^{1}}-\widehat{e_{1,1}^{1}}-\widehat{e_{1,2}^{n-1}}-\widehat{e_{2,1}^{n-1}}\right) .
$$

Proof. On the one hand,

$$
\begin{aligned}
\psi(U) & =\chi_{1}+\chi_{a^{n}}+\sum_{k=1, k \text { odd }}^{n-1} \epsilon^{n-k} E_{1,1}^{n-k}+\epsilon^{-(n-k)} E_{2,2}^{n-k} \\
& =\chi_{1}+\chi_{a^{n}}+\sum_{j=1, j \text { even }}^{n-1} \epsilon^{j}\left(\chi_{a^{j}}+\chi_{a^{n+j}}\right)+\epsilon^{-j}\left(\chi_{a^{n-j}}+\chi_{a^{2 n-j}}\right) \\
& =\sum_{j=0}^{2 n-1}(-1)^{j} \epsilon^{j} \chi_{a^{j}}=\sum_{j=0}^{2 n-1} \epsilon^{-(n-1) j} \chi_{a^{j}}=2 n \widehat{e_{1,1}^{n-1}} .
\end{aligned}
$$

On the other hand,

$$
\begin{aligned}
& \left(V+V^{*}\right)=2\left(-e_{3}-e_{4}\right)+\sum_{k=1, k \text { odd }}^{n-1}\left(\epsilon^{k}+\epsilon^{-k}\right)\left(r_{1,1}^{k}+r_{2,2}^{k}\right) \\
& \left(V-V^{*}\right)=\mathrm{i} \sum_{\mathrm{k}=1, \mathrm{k} \text { odd }}^{\mathrm{n}-1}\left(\epsilon^{\mathrm{k}}-\epsilon^{-\mathrm{k}}\right)\left(\mathrm{r}_{2,1}^{\mathrm{k}}-\mathrm{r}_{1,2}^{\mathrm{k}}\right) .
\end{aligned}
$$


It follows that:

$$
\begin{aligned}
\psi\left(V+V^{*}\right) & =2\left(-\chi_{b a^{n}}-\chi_{b}\right)+\sum_{k=1, k \text { odd }}^{n-1}\left(\epsilon^{k}+\epsilon^{-k}\right)\left(\chi_{b a^{n+k}}+\chi_{b a^{k}}+\chi_{b a^{2 n-k}}+\chi_{b a^{n-k}}\right) \\
& =-\sum_{j=0}^{2 n-1}\left(\epsilon^{(n-1) j}+\epsilon^{-(n-1) j}\right) \chi_{b a^{j}}=-2 n\left(\widehat{e_{1,2}^{n-1}}+\widehat{e_{2,1}^{n-1}}\right), \\
\psi\left(V-V^{*}\right) & =\sum_{k=1, k \text { odd }}^{n-1}\left(\epsilon^{k}-\epsilon^{-k}\right)\left(\chi_{a^{k}}+\chi_{a^{n-k}}-\chi_{a^{n+k}}-\chi_{a^{2 n-k}}\right) \\
& =\sum_{j=0}^{2 n-1}\left(\epsilon^{j}-\epsilon^{-j}\right) \chi_{a^{j}}=2 n\left(\widehat{e_{2,2}^{1}}-\widehat{e_{1,1}^{1}}\right),
\end{aligned}
$$

which gives the desired expression for $\psi(V)$. The expressions for $\psi\left(V+V^{*}\right)$ and $\psi\left(V-V^{*}\right)$ will be reused later on.

A.8.4. Coproducts of $\psi(U)$ and $\psi(V)$.

\section{Lemma A.8.4.}

$$
\Delta(\psi(U))=4 n^{2}\left(\widehat{e_{1,1}^{n-1}} \otimes \widehat{e_{1,1}^{n-1}}+\widehat{e_{1,2}^{n-1}} \otimes \widehat{e_{2,1}^{n-1}}\right) .
$$

$$
\begin{aligned}
\Delta(\psi(V))=2 n^{2} \widehat{\left(e_{2,2}^{1}\right.} \otimes \widehat{e_{2,2}^{1}} & +\widehat{e_{2,1}^{1}} \otimes \widehat{e_{1,2}^{1}}-\widehat{e_{1,1}^{1}} \otimes \widehat{e_{1,1}^{1}}-\widehat{e_{1,2}^{1}} \otimes \widehat{e_{2,1}^{1}} \\
& \left.-\widehat{e_{2,2}^{n-1}} \otimes \widehat{e_{2,1}^{n-1}}-\widehat{e_{2,1}^{n-1}} \otimes \widehat{e_{1,1}^{n-1}}-\widehat{e_{1,1}^{n-1}} \otimes \widehat{e_{1,2}^{n-1}}-\widehat{e_{1,2}^{n-1}} \otimes \widehat{e_{2,2}^{n-1}}\right)
\end{aligned}
$$

Proof.

$$
\begin{aligned}
\Delta(\psi(U)) & =\sum_{k=0}^{2 n-1} \sum_{j=0}^{2 n-1} \epsilon^{-(n-1) j}\left(\chi_{a^{k}} \otimes \chi_{a^{j-k}}+\chi_{a^{k} b} \otimes \chi_{b a^{j-k}}\right) \\
& =\sum_{k=0}^{2 n-1} \sum_{j=0}^{2 n-1} \epsilon^{-(n-1)(k+j)}\left(\chi_{a^{k}} \otimes \chi_{a^{j}}+\chi_{a^{k} b} \otimes \chi_{b a^{j}}\right) \\
& =4 n^{2}\left(\widehat{e_{1,1}^{n-1}} \otimes \widehat{e_{1,1}^{\widehat{n-1}}}+\widehat{e_{1,2}^{n-1}} \otimes \widehat{e_{2,1}^{n-1}}\right) .
\end{aligned}
$$

Similar calculations give:

$$
\begin{aligned}
\Delta\left(2 n \widehat{e_{2,2}^{1}}\right) & =4 n^{2}\left(\widehat{e_{2,2}^{1}} \otimes \widehat{e_{2,2}^{1}}+\widehat{e_{2,1}^{1}} \otimes \widehat{e_{1,2}^{1}}\right), \\
\Delta\left(2 n \widehat{e_{1,2}^{n-1}}\right) & =4 n^{2}\left(\widehat{e_{2,2}^{n-1}} \otimes \widehat{e_{2,1}^{n-1}}+\widehat{e_{1,2}^{n-1}} \otimes \widehat{e_{1,1}^{n-1}}\right) .
\end{aligned}
$$

The announced formula follows using the equalities $\widehat{e_{2,2}^{j}}={\widehat{e_{1,1}^{j}}}^{*}$ and $\widehat{e_{2,1}^{j}}={\widehat{e_{1,2}^{j}}}^{*}$.

A.8.5. Image of $\Delta(U)$ by $\psi \otimes \psi$. We now turn to the preservation by $\psi$ of the coproducts of $U$ and $V$, and therefore of $\lambda(a)=U+V$. Since $\lambda\left(a^{n+1}\right)=U-V$, we can calculate the coproducts of $U=1 / 2\left(\lambda(a)+\lambda\left(a^{n+1}\right)\right)$ and $V=1 / 2\left(\lambda(a)-\lambda\left(a^{n+1}\right)\right)$ using the expression for $\Omega$ for $n$ odd given in A.3.

Let us start with $U$ :

$$
\begin{aligned}
& \Delta(U)=\Omega(U \otimes U+V \otimes V) \Omega^{*} \\
& =\left(e_{1}+e_{2}+q_{1}+q_{2}\right) \otimes\left(e_{1}+e_{2}+q_{1}+q_{2}\right)(U \otimes U)\left(e_{1}+e_{2}+q_{1}+q_{2}\right) \otimes\left(e_{1}+e_{2}+q_{1}+q_{2}\right) \\
& +\left(e_{3}-i e_{4}-i p_{1}+p_{2}\right) \otimes\left(e_{3}+i e_{4}+i p_{1}+p_{2}\right)(V \otimes V)\left(e_{3}+i e_{4}+i p_{1}+p_{2}\right) \otimes\left(e_{3}-i e_{4}-i p_{1}+p_{2}\right) \\
& =U \otimes U+V^{\prime} \otimes V^{\prime *},
\end{aligned}
$$


where $V^{\prime}=\left(-e_{3}-e_{4}+\sum_{k=1, k \text { odd }}^{n-1} \epsilon^{k} r_{2,2}^{k}+\epsilon^{-k} r_{1,1}^{k}\right)$. The image of $V^{\prime}$ by $\psi$ is:

$$
\psi\left(V^{\prime}\right)=-\chi_{b a^{n}}-\chi_{b}+\sum_{k=1, k \text { odd }}^{n-1} \epsilon^{k} E_{2,2}^{k}+\epsilon^{-k} E_{1,1}^{k}=-\sum_{j=0}^{2 n-1} \epsilon^{(n-1) j} \chi_{b a^{j}}=-2 n \widehat{e_{1,2}} .
$$

It follows that $\psi\left(V^{*}\right)=-2 n \widehat{e_{2,1}^{n-1}}$, and we get:

$$
(\psi \otimes \psi)(\Delta(U))=4 n^{2}\left(\widehat{e_{1,1}^{n-1}} \otimes \widehat{e_{1,1}^{n-1}}+\widehat{e_{1,2}^{n-1}} \otimes \widehat{e_{2,1}^{n-1}}\right) .
$$

Using Lemma A.8.4, we conclude that $\psi$ preserves the coproduct of $U$.

A.8.6. Image of $\Delta(V)$ by $\psi \otimes \psi$. We now calculate the image of $\Delta(V)$ by $\psi \otimes \psi$ :

$$
\begin{aligned}
\Delta(V) & =\Omega(U \otimes V+V \otimes U) \Omega^{*}, \\
& =U_{1} \otimes V_{1}+U_{2} \otimes V_{2}+i\left(U_{3} \otimes V_{3}-U_{4} \otimes V_{4}\right) \\
& +V_{1} \otimes U_{1}+V_{2} \otimes U_{2}-i\left(V_{3} \otimes U_{3}-V_{4} \otimes U_{4}\right),
\end{aligned}
$$

where:

$$
\begin{gathered}
U_{1}=\left(e_{1}+q_{1}\right) U\left(e_{1}+q_{1}\right)=e_{1}+\sum_{k=1, k \text { even }}^{n-1} \frac{1}{2}\left(\epsilon^{k}+\epsilon^{-k}\right) p_{1,1}^{k}, \\
V_{1}=\left(e_{3}+e_{4}+p_{1}+p_{2}\right) V\left(e_{3}+e_{4}+p_{1}+p_{2}\right)=V, \\
U_{2}=\left(e_{2}+q_{2}\right) U\left(e_{2}+q_{2}\right)=e_{2}+\sum_{k=1, k \text { even }}^{n-1} \frac{1}{2}\left(\epsilon^{k}+\epsilon^{-k}\right) p_{2,2}^{k}, \\
V_{2}=\left(e_{3}-e_{4}-p_{1}+p_{2}\right) V\left(e_{3}-e_{4}-p_{1}+p_{2}\right)=V^{*}, \\
U_{3}=\left(e_{2}+q_{2}\right) U\left(e_{1}+q_{1}\right)=\sum_{k=1, k \text { even }}^{n-1} \frac{1}{4}\left(\epsilon^{k}-\epsilon^{-k}\right)\left(e_{1,1}^{k}+e_{1,2}^{k}-e_{2,1}^{k}-e_{2,2}^{k}\right), \\
V_{3}=\left(e_{3}-e_{4}-p_{1}+p_{2}\right) V\left(e_{3}+e_{4}+p_{1}+p_{2}\right)=-e_{3}+e_{4}-\sum_{k=1, k \text { odd }}^{n-1} \epsilon^{-k} e_{1,2}^{k}+\epsilon^{k} e_{2,1}^{k} \\
U_{4}=\left(e_{1}+q_{1}\right) U\left(e_{2}+q_{2}\right)=\sum_{k=1, k \text { even }}^{n-1} \frac{1}{4}\left(\epsilon^{k}-\epsilon^{-k}\right)\left(e_{1,1}^{k}-e_{1,2}^{k}+e_{2,1}^{k}-e_{2,2}^{k}\right), \\
V_{4}=\left(e_{3}+e_{4}+p_{1}+p_{2}\right) V\left(e_{3}-e_{4}-p_{1}+p_{2}\right)=-e_{3}+e_{4}-\sum_{k=1, k \text { odd }}^{n-1} \epsilon^{k} e_{1,2}^{k}+\epsilon^{-k} e_{2,1}^{k} .
\end{gathered}
$$

To split the calculation into more manageable chunks, we write:

$$
\begin{aligned}
& 2\left[U_{1} \otimes V_{1}+U_{2} \otimes V_{2}+i\left(U_{3} \otimes V_{3}-U_{4} \otimes V_{4}\right)\right]= \\
& \quad\left(U_{1}+U_{2}\right) \otimes\left(V_{1}+V_{2}\right)+\left(U_{1}-U_{2}\right) \otimes\left(V_{1}-V_{2}\right) \\
& \quad+\mathrm{i}\left[\left(\mathrm{U}_{3}-\mathrm{U}_{4}\right) \otimes\left(\mathrm{V}_{3}+\mathrm{V}_{4}\right)+\left(\mathrm{U}_{3}+\mathrm{U}_{4}\right) \otimes\left(\mathrm{V}_{3}-\mathrm{V}_{4}\right)\right]
\end{aligned}
$$




\section{Lemma A.8.6.}

$$
\begin{array}{ll}
\psi\left(U_{1}+U_{2}\right)=n\left(\widehat{e_{1,1}^{n-1}}+\widehat{e_{2,2}^{n-1}}\right), & \psi\left(U_{1}-U_{2}\right)=n\left(\widehat{e_{1,1}^{1}}+\widehat{e_{2,2}^{1}}\right), \\
\psi\left(U_{3}+U_{4}\right)=n\left(\widehat{e_{1,1}^{n-1}}-\widehat{e_{2,2}^{\widehat{n-1}}}\right), & \psi\left(U_{3}-U_{4}\right)=\mathrm{i} n\left(\widehat{e_{1,2}^{1}}-\widehat{e_{2,1}^{1}}\right), \\
\psi\left(V_{1}+V_{2}\right)=-2 n\left(\widehat{e_{1,2}^{n-1}}-\widehat{e_{2,1}^{n-1}}\right), & \psi\left(V_{1}-V_{2}\right)=2 n\left(\widehat{e_{2,2}^{1}}-\widehat{e_{1,1}^{1}}\right), \\
\psi\left(V_{3}+V_{4}\right)=2 n\left(\widehat{e_{1,2}^{1}}+\widehat{e_{2,1}^{1}}\right), & \psi\left(V_{3}-V_{4}\right)=2 \mathrm{i} n\left(\widehat{e_{1,2}^{n-1}}-\widehat{e_{2,1}^{n-1}}\right) .
\end{array}
$$

Proof.

$$
\begin{aligned}
\psi\left(U_{1}+U_{2}\right) & =\psi\left(e_{1}+e_{2}+\sum_{k=1, k \text { even }}^{n-1} \frac{1}{2}\left(\epsilon^{k}+\epsilon^{-k}\right)\left(e_{1,1}^{k}+e_{2,2}^{k}\right)\right) \\
& =\chi_{1}+\chi_{a^{n}}+\sum_{k=1, k \text { even }}^{n-1} \frac{1}{2}\left(\epsilon^{k}+\epsilon^{-k}\right)\left(\chi_{a^{n-k}}+\chi_{a^{2 n-k}}+\chi_{a^{k}}+\chi_{a^{n+k}}\right)=n\left(\widehat{e_{1,1}^{n-1}}+\widehat{e_{2,2}^{n-1}}\right), \\
\psi\left(U_{1}-U_{2}\right) & =\psi\left(e_{1}-e_{2}+\sum_{k=1, k \text { even }}^{n-1} \frac{1}{2}\left(\epsilon^{k}+\epsilon^{-k}\right)\left(e_{1,2}^{k}+e_{2,1}^{k}\right)\right) \\
& =\chi_{1}-\chi_{a^{n}}+\sum_{k=1, k \text { even }}^{n-1} \frac{1}{2}\left(\epsilon^{k}+\epsilon^{-k}\right)\left(-\chi_{a^{n-k}}+\chi_{a^{2 n-k}}+\chi_{a^{k}}-\chi_{a^{n+k}}\right)=n\left(\widehat{e_{1,1}^{1}}+\widehat{e_{2,2}^{1}}\right), \\
\psi\left(U_{3}+U_{4}\right) & =\psi\left(\sum_{k=1, k \text { even }}^{n-1} \frac{1}{2}\left(\epsilon^{k}-\epsilon^{-k}\right)\left(e_{1,1}^{k}-e_{2,2}^{k}\right)\right) \\
& =\sum_{k=1, k \text { even }}^{n-1} \frac{1}{2}\left(\epsilon^{k}-\epsilon^{-k}\right)\left(-\chi_{a^{n-k}}-\chi_{a^{2 n-k}}+\chi_{a^{k}}+\chi_{a^{n+k}}\right)=n\left(\widehat{e_{1,1}^{n-1}}-\widehat{e_{2,2}^{n-1}}\right) \\
\psi\left(U_{3}-U_{4}\right) & =\psi\left(\sum_{k=1, k \text { even }}^{n-1} \frac{1}{2}\left(\epsilon^{k}-\epsilon^{-k}\right)\left(e_{1,2}^{k}-e_{2,1}^{k}\right)\right), \\
& =\mathrm{i} \sum_{k=1, k \text { even }}^{n-1} \frac{1}{2}\left(\epsilon^{k}-\epsilon^{-k}\right)\left(\chi_{b a^{k}}-\chi_{b a^{2 n-k}}-\chi_{b a^{n+k}}+\chi_{b a^{n-k}}\right)=\hat{\mathrm{i} n\left(\widehat{e_{1,2}^{1}}-\widehat{e_{2,1}^{1}}\right) .}
\end{aligned}
$$

The expressions of $\psi\left(V_{1}+V_{2}\right)$ and $\psi\left(V_{1}-V_{2}\right)$ follow from Lemma A.8.3. Similarly, one calculates:

$$
\begin{aligned}
\psi\left(V_{3}+V_{4}\right) & =\psi\left(2\left(-e_{3}-e_{4}\right)+\sum_{k=1, k \text { odd }}^{n-1}\left(\epsilon^{k}+\epsilon^{-k}\right)\left(r_{1,2}^{k}+r_{2,1}^{k}\right)\right) \\
& =-2 \chi_{b a^{n}}+2 \chi_{b}+\sum_{k=1, k \text { odd }}^{n-1}\left(\epsilon^{k}+\epsilon^{-k}\right)\left(\chi_{b a^{k}}+\chi_{b a^{2 n-k}}-\chi_{b a^{n+k}}-\chi_{b a^{n-k}}\right) \\
& =2 n\left(\widehat{e_{1,2}^{1}}+\widehat{e_{2,1}^{1}}\right),
\end{aligned}
$$




$$
\begin{aligned}
\psi\left(V_{3}-V_{4}\right) & =\psi\left(\mathrm{i} \sum_{\mathrm{k}=1, \mathrm{k} \text { odd }}^{\mathrm{n}-1}\left(\epsilon^{\mathrm{k}}-\epsilon^{-\mathrm{k}}\right)\left(\mathrm{r}_{1,1}^{\mathrm{k}}-\mathrm{r}_{2,2}^{\mathrm{k}}\right)\right) \\
& =\mathrm{i} \sum_{k=1, k \text { odd }}^{n-1}\left(\epsilon^{k}-\epsilon^{-k}\right)\left(\chi_{b a^{n+k}}+\chi_{b a^{k}}-\chi_{b a^{2 n-k}}-\chi_{b a^{n-k}}\right) \\
& =2 \mathrm{i} n\left(e_{1,2}^{n-1}-e_{2,1}^{n-1}\right),
\end{aligned}
$$

which concludes the proof of this lemma.

We can at last check that the coproduct of $V$ is preserved:

$$
\begin{aligned}
n^{-2}(\psi \otimes \psi)(\Delta(V)) & \left.=-\left(\widehat{e_{1,1}^{n-1}}+\widehat{e_{2,2}^{n-1}}\right) \otimes\left(\widehat{e_{1,2}^{n-1}}+\widehat{e_{2,1}^{n-1}}\right)+\widehat{\left(e_{1,1}^{1}\right.}+\widehat{e_{2,2}^{1}}\right) \otimes\left(\widehat{e_{2,2}^{1}}-\widehat{e_{1,1}^{1}}\right) \\
& -\left(\widehat{e_{1,2}^{n-1}}+\widehat{e_{2,1}^{n-1}}\right) \otimes\left(\widehat{e_{1,1}^{n-1}}+\widehat{e_{2,2}^{n-1}}\right)+\left(\widehat{e_{2,2}^{1}}-\widehat{e_{1,1}^{1}}\right) \otimes\left(\widehat{e_{1,1}^{1}}+\widehat{e_{2,2}^{1}}\right) \\
& -\left(\widehat{e_{1,2}^{1}}-\widehat{e_{2,1}^{1}}\right) \otimes\left(\widehat{e_{1,2}^{1}}+\widehat{e_{2,1}^{1}}\right)-\left(\widehat{e_{1,1}^{n-1}}-\widehat{e_{2,2}^{n-1}}\right) \otimes\left(\widehat{e_{1,2}^{n-1}}-\widehat{e_{2,1}^{n-1}}\right) \\
& +\left(\widehat{e_{1,2}^{1}}+\widehat{e_{2,1}^{1}}\right) \otimes\left(\widehat{e_{1,2}^{1}}-\widehat{e_{2,1}^{1}}\right)+\left(\widehat{e_{1,2}^{n-1}}-\widehat{e_{2,1}^{n-1}}\right) \otimes\left(\widehat{e_{1,1}^{n-1}}-\widehat{e_{2,2}^{n-1}}\right) \\
& =n^{-2} \Delta(\psi(V)) .
\end{aligned}
$$




\section{Appendix B. Computer exploration with MuPaD-Combinat}

Most of the research we report on in this paper has been driven by computer exploration. In this section, we quickly describe the tools we designed, implemented, and used, present typical computations, and discuss some exploration strategies. To this end, we use the construction of all coideal subalgebras of $K D(3)$ as running example. We recommend to start by skimming through the demonstration, in order to get a rough idea of what computations are, or not, achievable.

B.1. Software context. Our work is based on MuPAD-Combinat [HT04, an open-source algebraic combinatorics package for the computer algebra system MuPAD [The96]. Among other things, it provides a high-level framework for implementing Hopf algebras and the like. All the extensions we wrote for this research are publicly available from the developers repository (see http://mupad-combinat.sf.net/); in fact, the two authors used that mean to share the code between them. With it, new finite dimensional Kac algebras obtained by deformation of group algebras may be implemented concisely (the full implementation of the Kac-Paljutkin algebra takes about 50 lines of code, including comments). Most of the code is fairly generic and already integrated in the MuPAD-Combinat core, and was beneficial to several unrelated research projects. The remaining specific code is provided as a separate worksheet. Feel free to contact the second author for help.

Since then, the MuPAD-Combinat project was migrated to the completely open-source platform Sage $\left[\mathrm{S}^{+} 09, \mathrm{SCc} 08\right]$. Specific extensions, like this one, are migrated according to their usefulness for new research projects.

B.2. Setup. The first step is to start a new MuPAD session, and to setup the stage for the computations (think of it as the preliminaries section of a research paper which defines short hand notations). We load the MuPAD-Combinat package by issuing:

>> package ("Combinat")

Next we load a worksheet which contains code and short-hand notations specific to that specific research project. For the user convenience, a short help will be displayed.

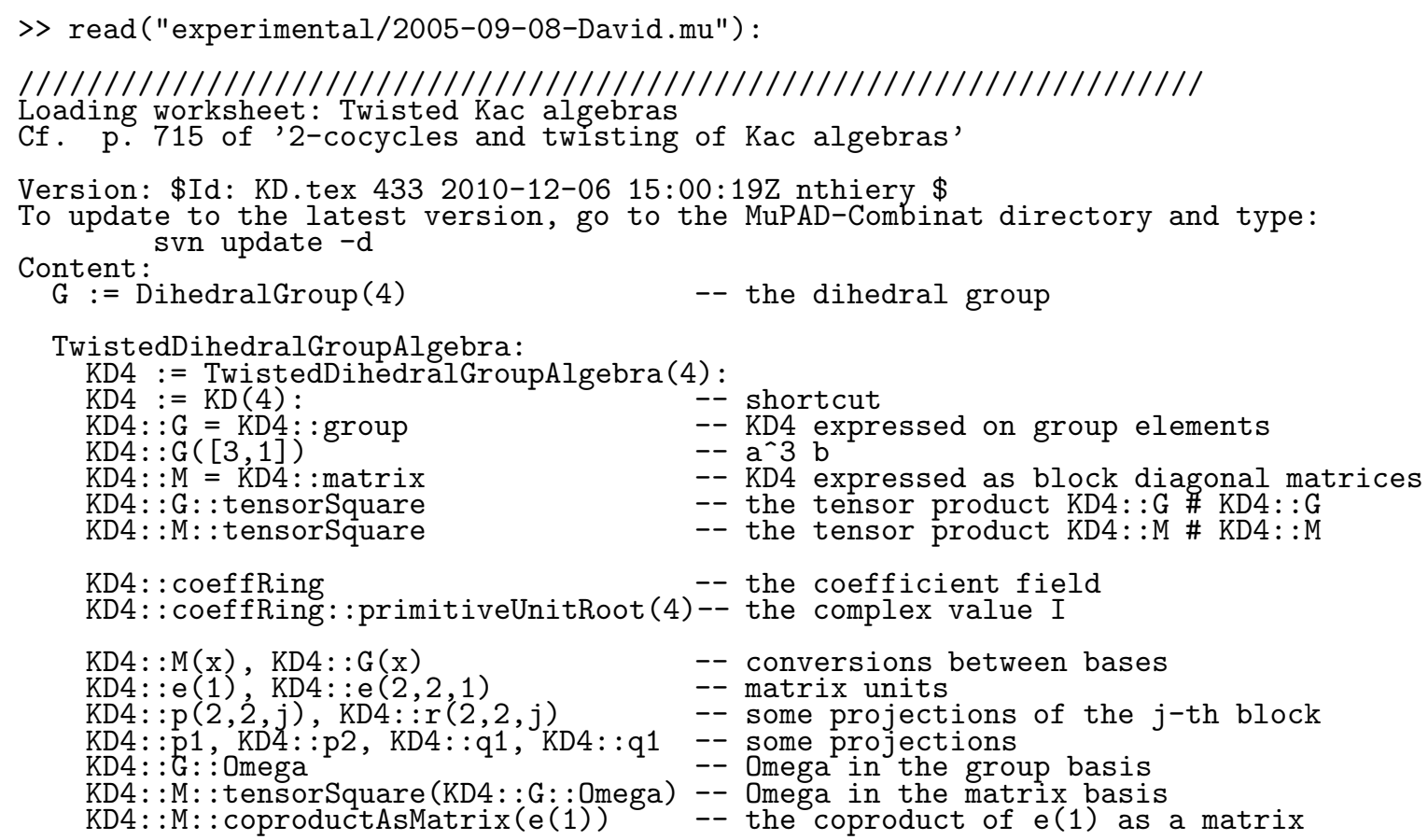




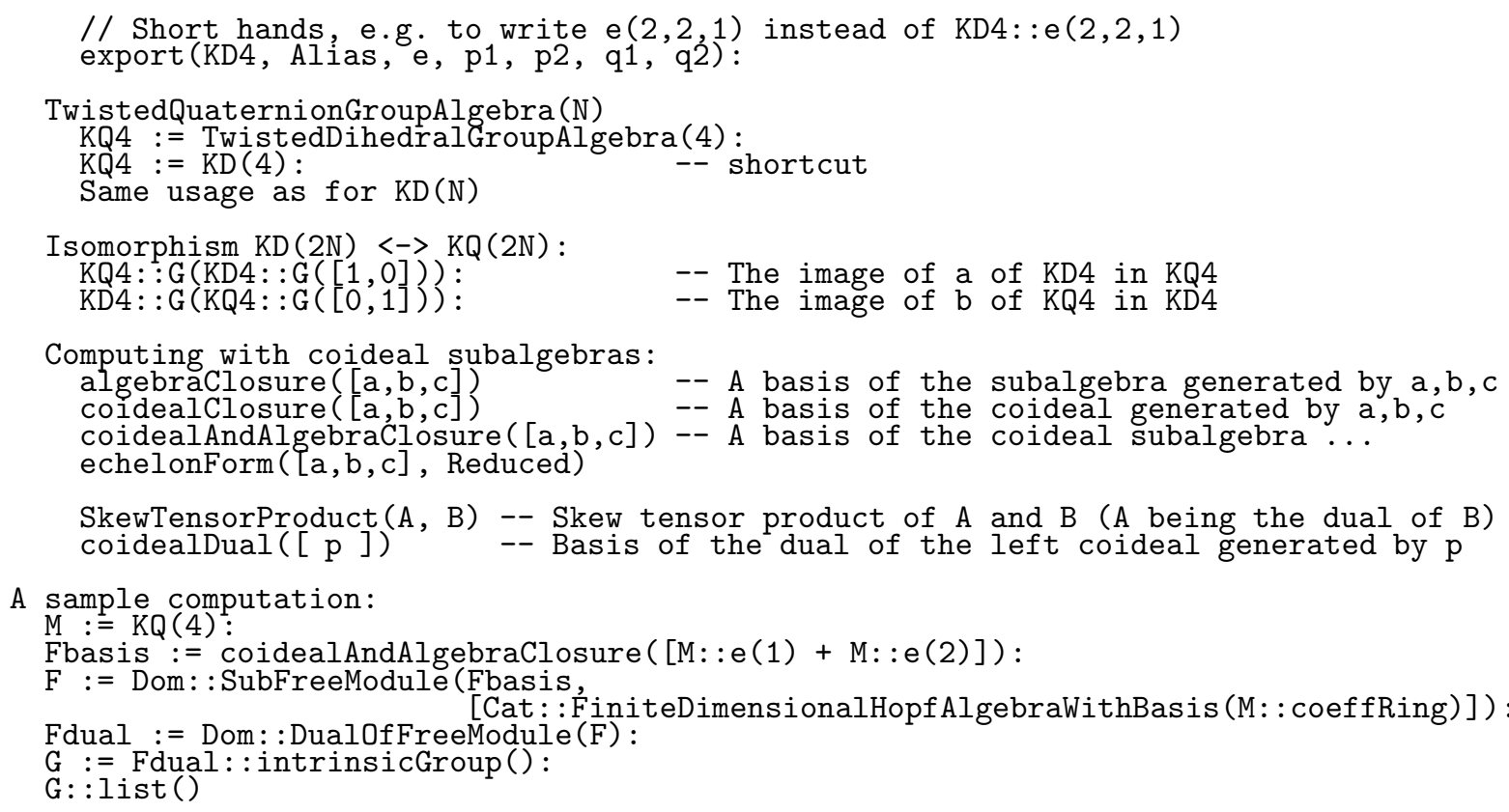

//////////////////////////////////////////////////////////////

Mind that this worksheet is experimental; for further help one needs to dig into the code. On the other hand, all features that are integrated into MuPAD-Combinat or MuPAD are documented within the usual MuPAD help system.

B.3. Computing with elements. Let us define $K D(3)$ :

$>\mathrm{KD} 3:=\mathrm{KD}(3)$ :

and shortcuts to its generators:

> [aa,bb] := KD3: :group: :algebraGenerators: :list()

$$
[B(a), B(b)]
$$

Now we can use MuPAD as a pocket calculator:

$>\mathrm{bb}^{\wedge} 2$

$$
B(1)
$$

The point is that MuPAD knows that bb lies in $K D(3)$ (more precisely, the object bb is in the domain KD3: : group ${ }^{10}$ ), and therefore applies the corresponding computation rules (usual object oriented programming paradigm). Here are some further simple computations:

> a $a^{\wedge} 2, \mathrm{aa}^{\wedge} 6, \mathrm{bb} * \mathrm{aa}$

$$
B\left(a^{2}\right), B(1), B\left(a^{5} b\right)
$$

and a more complicated one:

$$
\begin{aligned}
& >\left(1-\mathrm{aa}^{\wedge} 3\right) *\left(1+\mathrm{aa}^{\wedge} 3\right)+1 / 2 * \mathrm{bb}^{\wedge} * \mathrm{aa}^{\wedge} 3 \\
& 1 / 2 B\left(a^{3} b\right)
\end{aligned}
$$

Note that all computations above are done in the group algebra. Namely, KD3: : group (or KD3: : G) models the concrete algebra $K D(3)$ with its elements expanded on the group basis. However, $K D(3)$ can also be represented as a block-matrix algebra, with matrix units as basis, and it is often more convenient or efficient to do the computations there.

\footnotetext{
${ }^{10}$ In MuPAD parlance, concrete classes are called domains
} 
This basis is modeled by the domain KD3: : matrix (or KD3: :M for short), and the change of basis is done in the natural way:

> KD3: :M(aa $+2 * b b)$

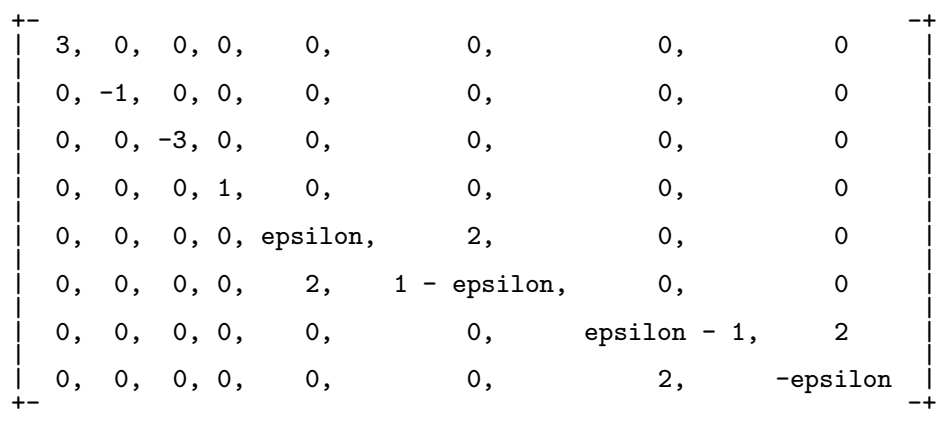

Some comments are in order:

- An element of KD: : M is displayed as a single large matrix; however, the four $1 \times 1$ blocks and the three $2 \times 2$ blocks inside are well visible in the example above.

- So far, we have not specified the ground field. It must be of characteristic zero, and contain some roots of unity to define $\Omega$ (see A.3) and the left regular representation (see A.1). In theory one can just take $\mathbb{C}$, but in practice one needs a computable field. By default, an appropriate algebraic extension of $\mathbb{Q}$ is automatically constructed:

> KD3: : coeffRing

$$
\text { Q(II, epsilon) }
$$

where $\mathrm{II}^{4}=1$, and epsilon ${ }^{6}=1$.

- The basis change is implemented by specifying the images of $a$ and $b$ and stating that it is an algebra morphism. The inverse basis change is deduced automatically by matrix inversion. Appropriate caching is done to avoid computation overhead. This is completely transparent to the user, and mostly transparent for the developer (encapsulation principle).

- We show here the MuPAD output in the text interface. In the graphical interface things look better; in particular, epsilon could be typeset as $\epsilon$.

So far, we have only played with the algebra structure of $K D(3)$ which is just the usual group algebra structure. Let us compute some coproducts, starting with some group like elements (note: tensor products are denoted by the symbol \#):

$>$ coproduct ( $\left.\mathrm{aa}^{\wedge} 3\right), \operatorname{coproduct}(\mathrm{bb})$

$$
B\left(a^{3}\right) \# B\left(a^{3}\right), B(b) \# B(b)
$$

Here is the coproduct of $a$ :

$>$ coproduct (aa)

$$
\begin{gathered}
1 / 16 \mathrm{~B}\left(\mathrm{a}^{4} \mathrm{~b}\right) \# \mathrm{~B}\left(\mathrm{a}^{4} \mathrm{~b}\right)+\backslash-\frac{\mathrm{II}}{8}-1 / 16 \backslash \mathrm{B}\left(\mathrm{a}^{4} \mathrm{~b}\right) \# \mathrm{~B}\left(\mathrm{a}^{5}\right)+ \\
\ldots \text { one hundred lines sniped out } \ldots \\
-1 / 16 \mathrm{~B}(\mathrm{a}) \# \mathrm{~B}\left(\mathrm{a}^{2}\right)+7 / 16 \mathrm{~B}(\mathrm{a}) \# \mathrm{~B}(\mathrm{a})
\end{gathered}
$$

The implementation of the coproduct follows closely Vainerman's definition Vai98 by deformation of the usual coproduct. In particular, it goes through the definition of $\omega$ and $\Omega$ : 


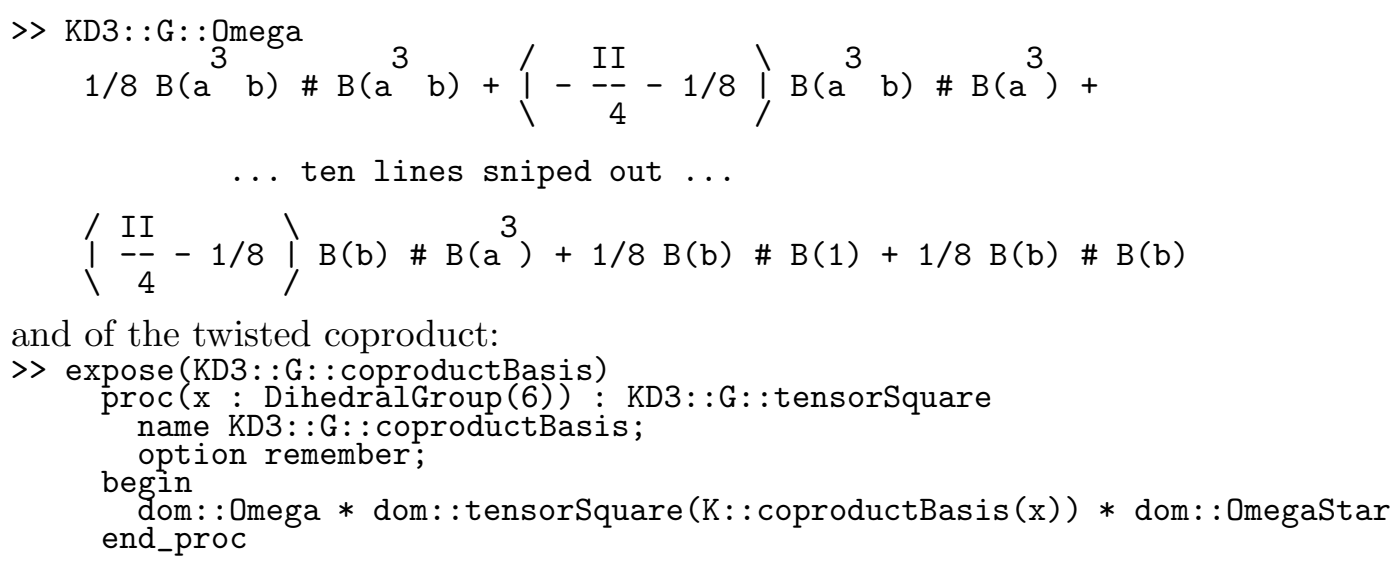

This function just computes the image of a basis element, and the actual coproduct is obtained by linearity. Thanks to the option remember, the computation is done only once. dom is a place holder for the current domain (here $K D(n)$ ), and $\mathrm{K}$ denotes the original Kac algebra (here $\mathbb{C}\left[D_{6}\right]$ ). The code is generic, and can be used to twist any Kac algebra by an appropriate cocycle. KD3: :M: : tensorSquare and KD3: : G: : tensorSquare model $K D(3) \otimes K D(3)$ respectively in the group and the matrix basis. The changes of basis between the two are defined automatically, and registered as implicit conversions. Those conversions are used transparently to compute coproducts in the matrix basis.

B.4. Computing with coideal subalgebras. A foremost feature that we used for exploration was the ability to compute properties of coideal subalgebras generated by various elements, in the hope to find Jones projections. Let us first define a shortcut for the matrix units:

$>$ e :=KD3: :e:

Now, $e_{i}$ and $e_{i, j}^{k}$ are given respectively by e(i) and $\mathrm{e}(\mathrm{i}, \mathrm{j}, \mathrm{k})$. We compute the coideal subalgebra $K_{1}$ generated by the projection $e_{1}+e_{2}+e_{3}+e_{4}$ :

$>$ K1basis := coidealAndAlgebraClosure $([\mathrm{e}(1)+\mathrm{e}(2)+\mathrm{e}(3)+\mathrm{e}(4)])$

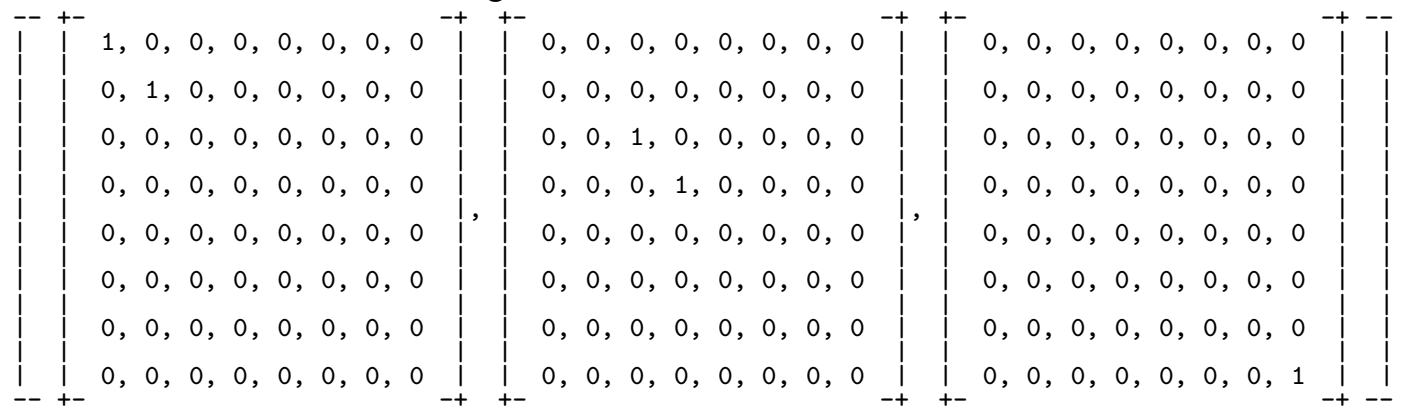

The result is a basis of $K_{1}$ in echelon form. Its dimension is consistent with the trace of $e_{1}+e_{2}+e_{3}+e_{4}$ :

$\gg 1 /(\mathrm{e}(1)+\mathrm{e}(2)+\mathrm{e}(3)+\mathrm{e}(4)):: \underset{3}{\operatorname{traceNormalized}()}$

It follows that $e_{1}+e_{2}+e_{3}+e_{4}$ is the Jones projection $p_{K_{1}}$ (see Remark 2.8.2). 
To give a flavor of the implementation work, here is the code for computing algebra and coideal subalgebra closures. It is defined generically for any domain implementing the appropriate operations (dom is a place holder for the current domain):

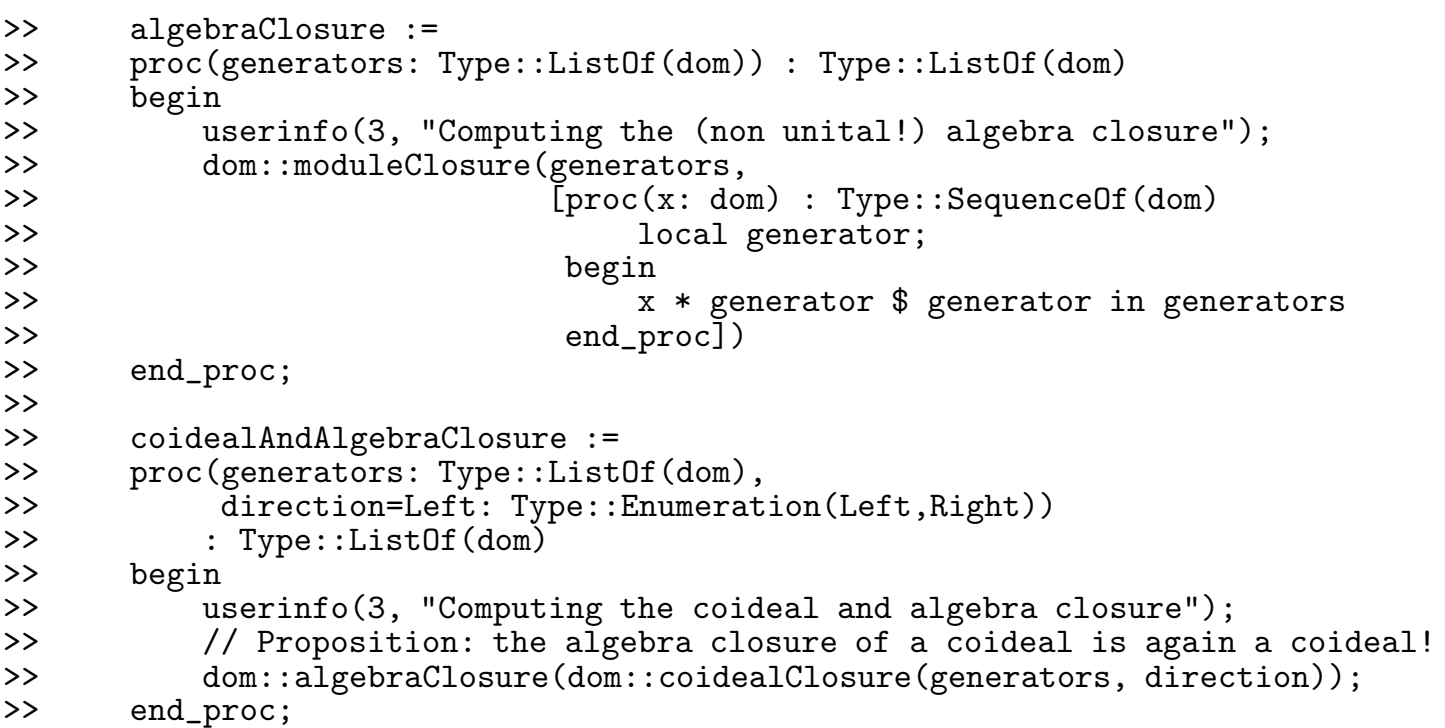

In short: thanks to the underlying computer science work in the design and implementation of the platform, the algorithms may be written in a reasonnably expressive and mathematically meaningful way.

B.4.1. The coideal subalgebra $K_{2}$ and subalgebras of functions on a group. Consider now the coideal subalgebra $K_{2}$ generated by the projection $e_{1}+e_{2}$ of trace $1 / 6$ :

$>\mathrm{K} 2 \mathrm{basis}:=$ coidealAndAlgebraClosure $([\mathrm{e}(1)+e(2)])$ :

$\gg$ nops(K2basis)

6

It has the desired dimension, so that $e_{1}+e_{2}$ is the Jones projection of $K_{2}$.

Consider now the coproduct of $e_{1}+e_{2}$ :

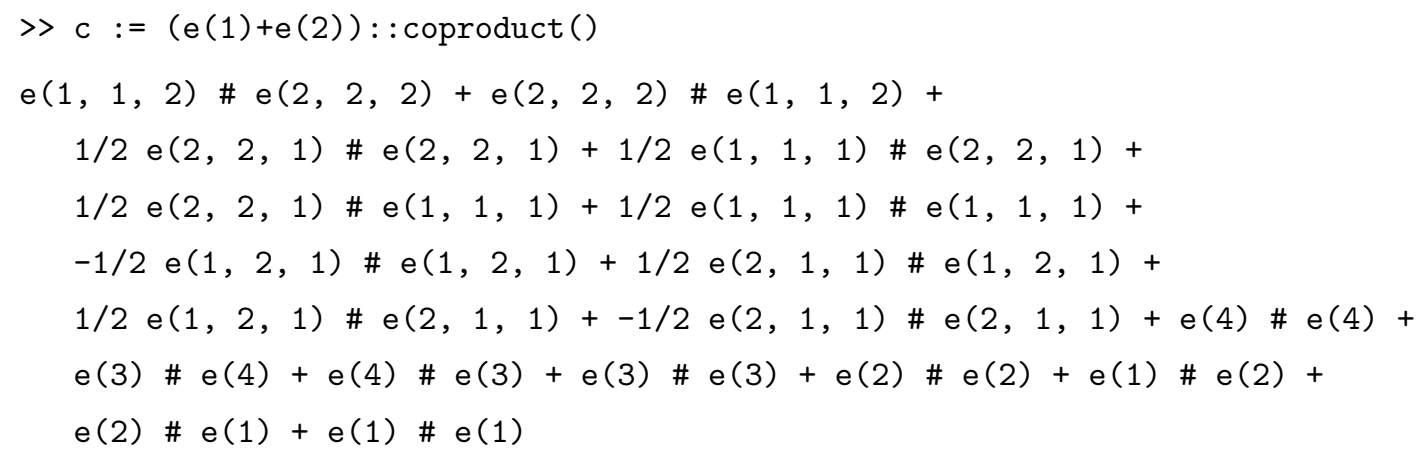

It turns out to be symmetric:

> c - c: :mapsupport (revert)

0

and therefore $K_{2}$ is a Kac subalgebra, the properties of which we now investigate. To this end, we define the subspace spanned by this basis, and claim to MuPAD that it is indeed a Kac subalgebra: 
$>$ K2 := Dom: :SubFreeModule(K2basis,

$>\quad$ [Cat::FiniteDimensionalHopfAlgebraWithBasis(KD3: : coeffRing)]):

The computation rules inside K2 are then inherited from those of KD3: :M. We first ask whether $K_{2}$ is commutative or cocommutative:

> K2: : isCommutative(), K2: : isCocommutative()

TRUE, FALSE

This tells us that $K_{2}$ is the dual of the algebra $\mathbb{C}[G]$ of some non commutative group $G$. To find $G$, we first define the dual of $K_{2}$ :

> K2dual := K2::Dual():

As expected, there are six group like elements in it:

>> K2dual: :groupLikeElements()

$$
\begin{aligned}
& {[- \text { II } \bar{B}([6,5])+\bar{B}([5,5]), \text { II } \bar{B}([6,5])+\bar{B}([5,5]), \bar{B}([7,7]),} \\
& \quad \bar{B}([8,8]), \bar{B}([1,1]), \bar{B}([3,3])]
\end{aligned}
$$

They are expressed in the dual basis of the row reduced echelon basis for K2; this representation is not very much useful. On the other hand, we may instead consider all of them together as the instrisic group:

> intrinsicGroup := K2dual: : intrinsicGroup():

and test that it is isomorphic to the dihedral group $D_{3}$ :

$>$ D3 := DihedralGroup(3):

> nops (D3: : groupEmbeddings (intrinsicGroup()))

6

The algorithmic behind this last step is currently simplistic. It could not deal with large groups like other specialized software like GAP could, but is sufficient for our purpose. On the other hand, the computation of the group-like elements themselves is rather efficient: it is done by computing the rank one central projections in the dual algebra. More generally, we can compute the full representation theory of finite dimensional algebras. For example:

>> K2dual: : isSemiSimple()

TRUE

K2dual: : simpleModulesDimensions()

$[2,1,1]$

B.4.2. Coidalgebras in $K_{2}$. We now show how to construct the Jones projections of the coideal subalgebras of $K_{2}=L^{\infty}\left(D_{3}\right)$, which are in correspondence with the subgroups of $D_{3}$ (see 2.8.4 and 5.7). Here we consider as example a subgroup $Z_{2}$ of order 2 of $D_{3}$. Take the second generator of the intrinsic group of $\widehat{K_{2}}$, and write it as an element of $\widehat{K_{2}}$ :

$>c:=$ intrinsicGroup $([2])$;

$\gg c:=c:: \operatorname{lift}()$ 
Here is the subgroup it generates:

>> Z2 := K2dual: :multiplicativeClosure([c])

$$
[B([5,5])+-\operatorname{II~} B([6,5]), B([1,1])]
$$

The corresponding coideal subalgebra $I$ consists of the functions on $D_{3}$ which are constant on right cosets for $Z_{2}$; it is of dimension $\left[D_{3}: Z_{2}\right]=3$. The Jones projection is given by the formula $\sum_{g \in Z_{2}} \widehat{g}$ (see 2.8.4):

> pI := _plus ( g: :groupLikeToIdempotentOfDual() \$ g in Z2 ):

The result is actually given in $K_{2}$; we lift it to an element of $K D(3)$ :

> pI := pI: :toSupModule()

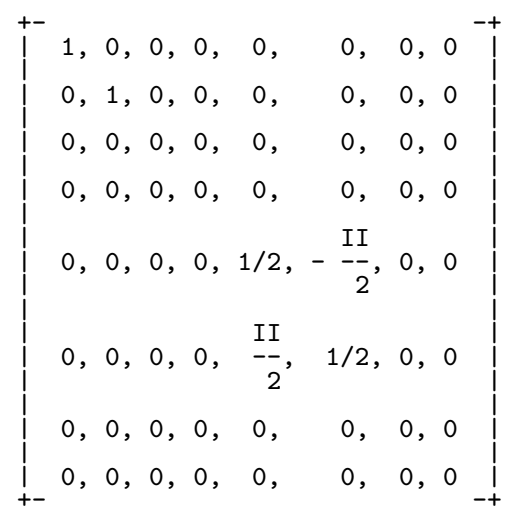

This gives us $p_{K_{21}}=e_{1}+e_{2}+r_{1,1}^{1}$, as in 6.9.1.

As a double check, here is the basis of the coideal $K_{21}$ it generates:

> coidealAndAlgebraClosure( $[\mathrm{pI}]$ )

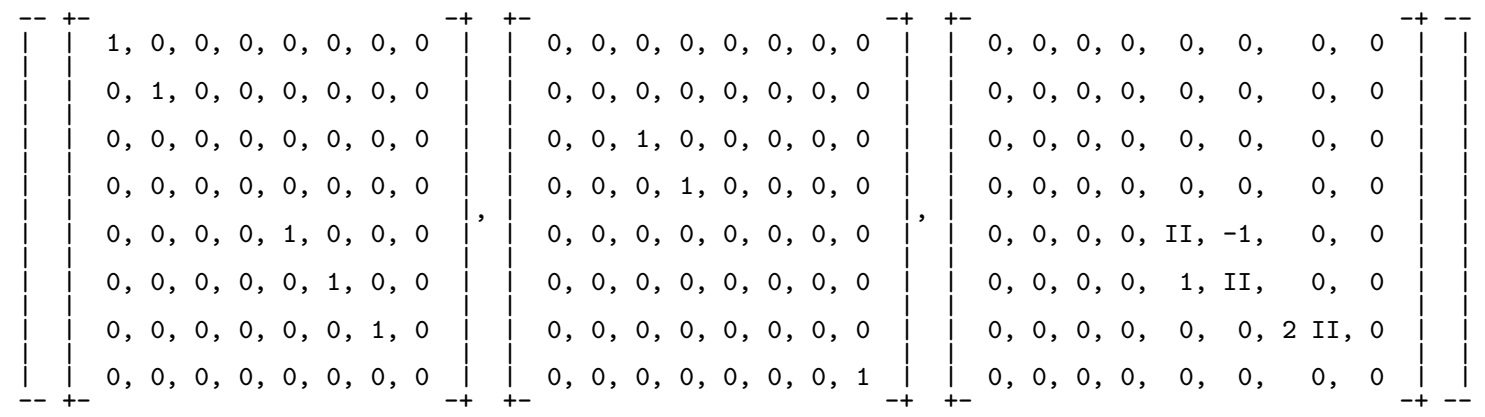

which is consistent with the trace of the projection:

>> 1 / pI: :traceNormalized()

3

Doing this for all subgroups of $D_{3}$ yields all coideal subalgebras of $K D(3)$ of dimension 2 and 3 .

B.4.3. Deeper study of $K_{2}$, and generalization to $K D(n)$. Studying $K_{2}$ this way had the advantage to be completely automatic. However to generalize the results to any $K D(n)$, we need to have a closer look at the structure, and do some things manually. Here, we are lucky enough that the row reduced basis comes out quite nicely (this is seldom the case): 
K2basis := coidealAndAlgebraClosure $([e(1)+e(2)])$

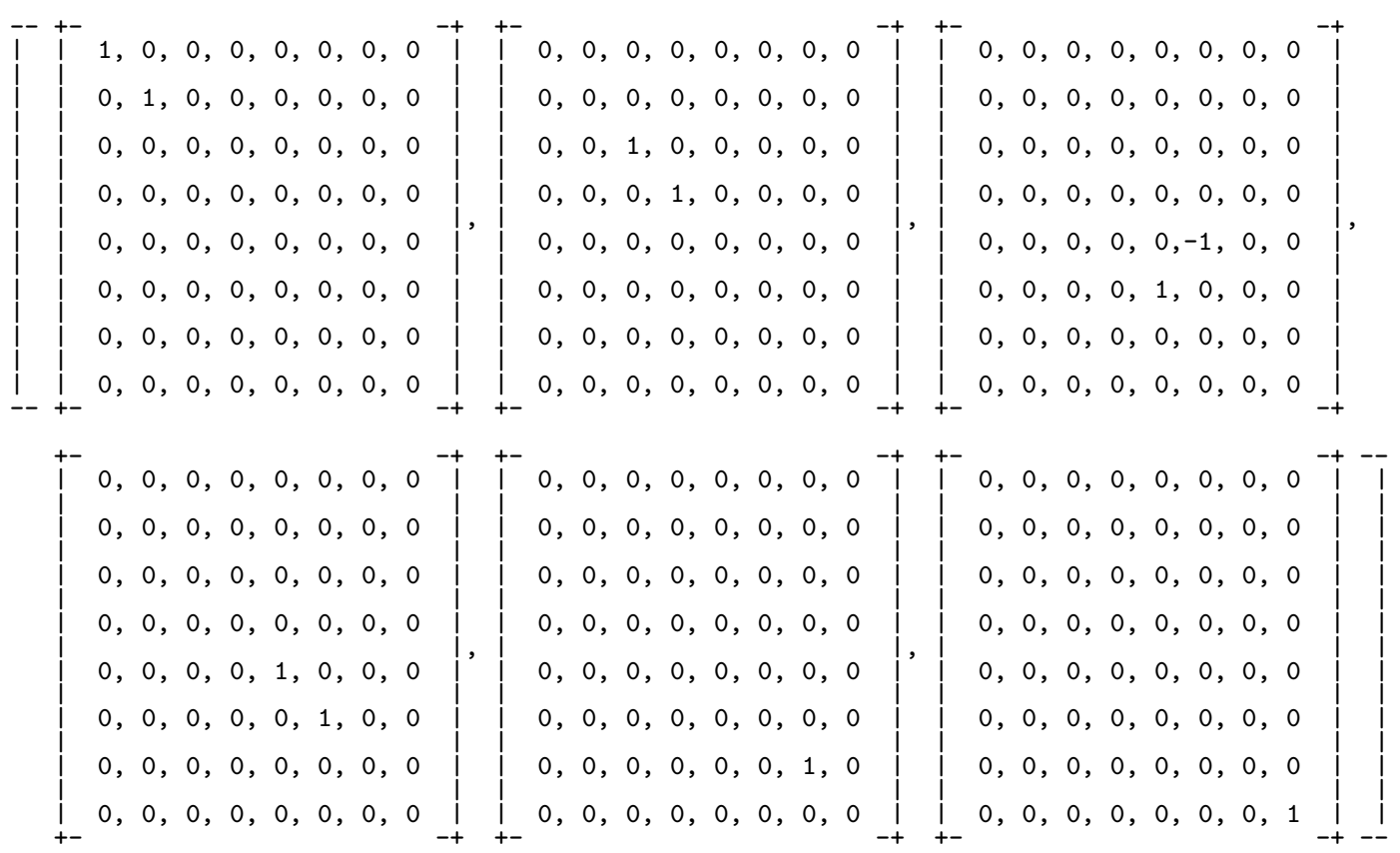

In particular, we can read off this basis the complete algebra structure of $K_{2}$ : it is a commutative algebra whose minimal projections are easy to find.

The expression of the coproduct of $e_{1}+e_{2}$ did not look very good. As usual in computer exploration, an essential issue is to find the right view where the output is readable and exploitable by a human; customizable outputs are therefore at a premium. For example the first author's favorite view for a tensor element (which tends to be huge) is as a matrix $M=\left(m_{a, b}\right)_{a, b}$ whose rows and columns are indexed by the matrix units of $K D(m)$ (here, for $m=3$ : $\left.\left(e_{1}, \ldots, e_{4}, e_{1,1}^{1}, e_{2,2}^{1}, e_{1,2}^{1}, e_{2,1}^{1}\right)\right)$, and where $m_{a, b}$ is the coefficient of $a \otimes b$. With this view, the coproduct of $e_{1}+e_{2}$ now also comes out nicely (see also A.5.2):

>> KD3: :M: : tensorElementToMatrix $((e(1)+e(2)):: \operatorname{coproduct}())$

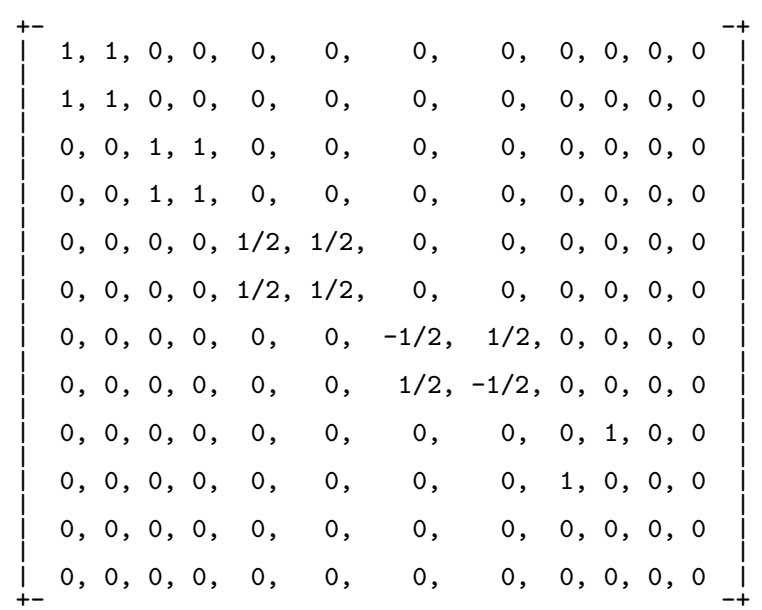

For example, it becomes obvious that this coproduct is symmetric, implying that $K_{2}$ is a Kac subalgebra (see 2.8.3); as it is commutative, we have $K_{2} \equiv L^{\infty}(G)$ for some group $G$ (see 2.8.4). Note that a basis for $K_{2}$ can also be read off the rows. Using duality, the elements of $G$ are given by the rank 1 central projections. With a bit more work, one can 
deduce from the expression of $\Delta\left(e_{1}+e_{2}\right)$ all the pairs $\left(g, g^{-1}\right)$ of inverse elements in $G$. Looking at some other coproducts reveals the complete group structure of $G$ (see 5.7).

To generalize this we look at $n=5$ :

$>\mathrm{K}:=\mathrm{KD}(5):$

> K: :M: :tensorElementToMatrix $((\mathrm{K}:: \mathrm{e}(1)+\mathrm{K}:: \mathrm{e}(2)):: \operatorname{coproduct}())$

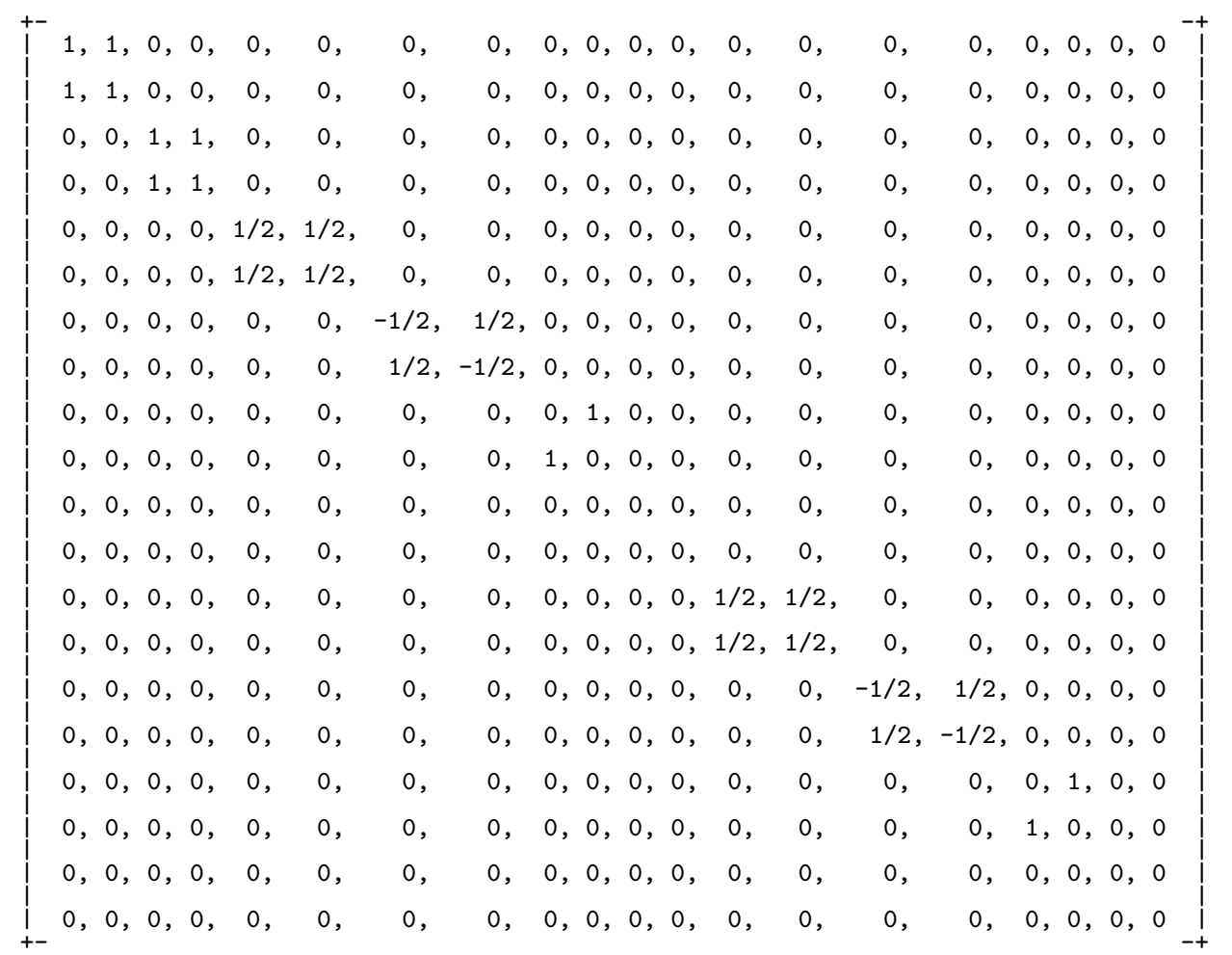

This suggests the general formulas of A.5.2 which we can double check for other values of $n$ before starting to prove them:

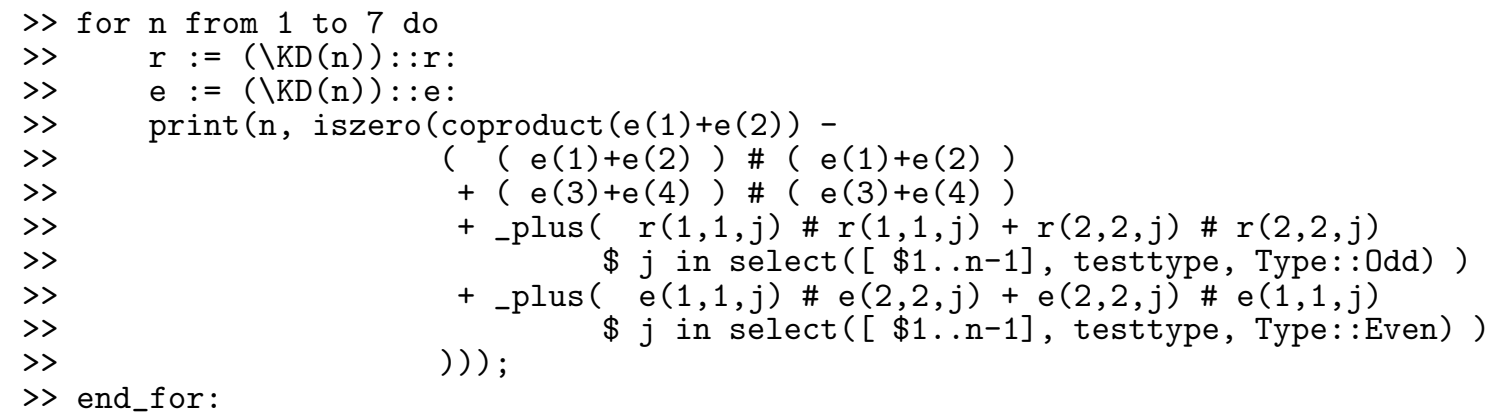

$$
\begin{aligned}
& \text { 1, } \text { TRUE } \\
& 7, \text { TRUE } \\
& \text { TRUE }
\end{aligned}
$$

With some patience and perseverance, the other required coproducts can be reverse engineered the same way for all $n$.

B.5. Computing Kac isomorphisms and applications. We now demonstrate the use of algorithm 5.5 .8 to compute automorphisms, self-duality, and isomorphisms, with the search of coideals as motivation. 
B.5.1. The coideal subalgebras $K_{3}$ and $K_{4}$, and automorphisms of $K D(3)$. Computing the coproducts of $e_{1}+e_{3}$ and $e_{1}+e_{4}$ as above shows that the corresponding coideal subalgebras $K_{3}$ and $K_{4}$ are not Kac subalgebras. However, they look similar, and it is natural to ask whether there exists an automorphism of $K D(3)$ which would exchange them. To this end, we compute the automorphism group of $K D(3)$ (see 5.5.8):

> automorphismGroup := KD3: :G: :algebraEmbeddings (KD3::G) :

A few minutes and a tea later, we obtain four automorphisms:

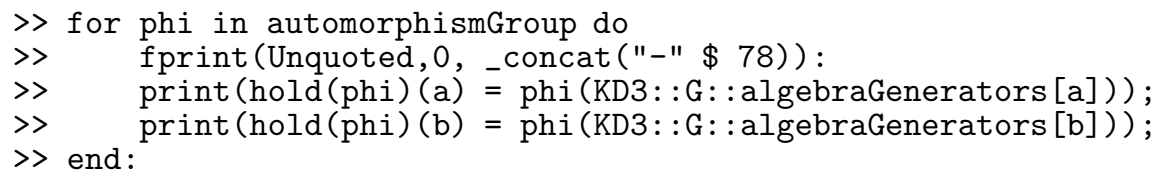

$\operatorname{phi}(\mathrm{a})=1 / 2 \mathrm{~B}\left(\mathrm{a}^{5}\right)+-1 / 2 \mathrm{~B}\left(\mathrm{a}^{4}\right)+1 / 2 \mathrm{~B}\left(\mathrm{a}^{2}\right)+1 / 2 \mathrm{~B}(\mathrm{a})$
$\operatorname{phi}(\mathrm{b})=\mathrm{B}\left(\mathrm{a}^{3} \mathrm{~b}\right)$
$\operatorname{phi}(\mathrm{a})=1 / 2 \mathrm{~B}\left(\mathrm{a}^{5}\right)+1 / 2 \mathrm{~B}\left(\mathrm{a}^{4}\right)+-1 / 2 \mathrm{~B}\left(\mathrm{a}^{2}\right)+1 / 2 \mathrm{~B}(\mathrm{a})$
$\operatorname{phi}(\mathrm{b})=\mathrm{B}\left(\mathrm{a}^{3} \mathrm{~b}\right)$
$\operatorname{phi}(\mathrm{a})=\mathrm{B}\left(\mathrm{a}^{5}\right)$
$\operatorname{phi}(\mathrm{b})=\mathrm{B}(\mathrm{b})$
$\operatorname{phi}(\mathrm{a})=\mathrm{B}(\mathrm{a})$
$\operatorname{phi}(\mathrm{b})=\mathrm{B}(\mathrm{b})$

Half of them are obviously induced by automorphisms of the group which fix $H$. The other half are obtained from $\Theta^{\prime}$ :

>> ThetaPrime := automorphismGroup [1]:

which is an involution:

ThetaPrime(ThetaPrime(KD3: :G: :algebraGenerators [a])),

$>$ ThetaPrime(ThetaPrime(KD3: :G: :algebraGenerators [b] ))

$$
B(a), B(b)
$$

The generalization of the formula for $\Theta^{\prime}$ to any $n$ is straightforward (see Proposition 5.5.2).

Going back to our original problem, we see that $\Theta^{\prime}$ exchanges $e_{1}+e_{3}$ and $e_{1}+e_{4}$ :

>> KD3: :M(ThetaPrime (KD3::G( KD3::e(1) + KD3::e(3))))

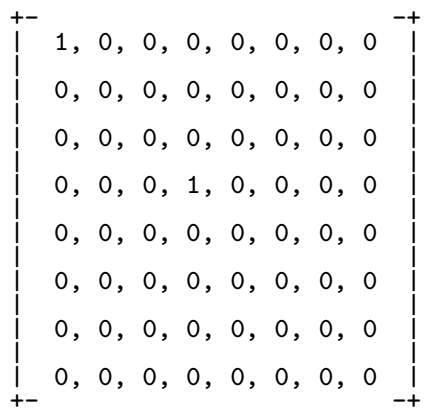

Therefore $K_{3}$ and $K_{4}$ are isomorphic (see 6.3.1). 
$>$ MdualToGdual := KD3: :G: :dualOfModuleMorphism(KD3::M) :

> Phi := MdualToGdual @ psi @ KD3::G:

$>\operatorname{Phi}(\mathrm{KD} 3:: \mathrm{e}(2,1,1))$

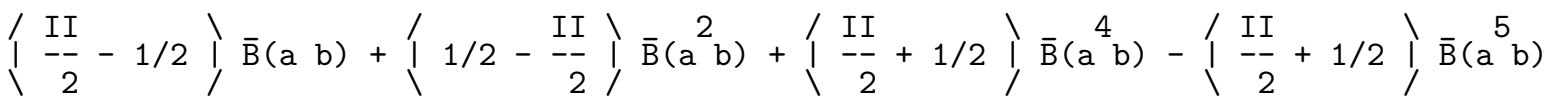

B.5.3. Coidalgebras of dimension 4 and the antiisomorphism $\delta$. We now use the isomorphism of the previous section to construct explicitly the coideals of dimension 4 of $K D(3)$. Consider for example the coideal $K_{21}$ of dimension 3 whose Jones projection we computed in B.4.2. We construct a basis for $\delta\left(K_{21}\right)$ in $\widehat{K D(3)}$ by looking for the commutant of $p_{K_{21}}$ in $\widehat{K D(3)} \subset \widehat{K D(3)} \rtimes K D(3)$.

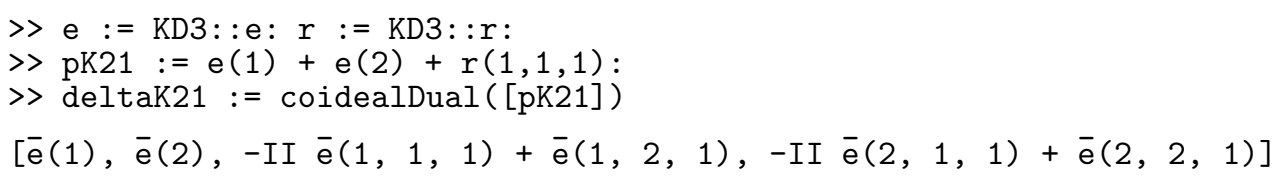

Using the inverse of the isomorphism $\Phi$ :

$\gg$ psi := KD3: :toDualIsomorphism:

> psiInv := KD3::G::inverseOfModuleMorphism(psi):

we identify $\delta\left(K_{21}\right)$ as a coideal $J_{20}$ of $K D(3)$ :

> J20 := map (deltaK21, psiInv)

$$
I_{--} B(1), B\left(a^{3}\right),\left|-\frac{I I}{2}-1 / 4\right| B\left(a^{5} b\right)+\frac{I I}{4} B\left(a^{5}\right)+1 / 4 B\left(a^{4} b\right)+\frac{I I}{4} B\left(a^{4}\right)+
$$$$
\left\{1 / 4-\frac{I I}{2} \mid B\left(a^{2} b\right)+-\frac{I I}{4} B\left(a^{2}\right)+-1 / 4 B(a b)+-\frac{I I}{4} B(a),\right.
$$$$
\left|\frac{I I}{4}+1 / 2\right| B\left(a^{5} b\right)+1 / 4 B\left(a^{5}\right)+-\frac{I I}{4} B\left(a^{4} b\right)+1 / 4 B\left(a^{4}\right)+
$$$$
\left\{1 / 2-\frac{I I}{4} \mid B\left(a^{2} b\right)+-1 / 4 B\left(a^{2}\right)+\frac{I I}{4} B(a b)+-1 / 4 B(a)\right.
$$

Here, we are lucky enough that the obtained basis for $J_{20}$ is orthonormal:

$\gg \operatorname{matrix}(4,4,(i, j) \rightarrow \operatorname{scalar}(\mathrm{J} 20[i], \mathrm{J} 20[\mathrm{j}]))$

$$
\left|\begin{array}{cccc}
+- & 0, & 0, & 0 \\
0, & 1, & 0, & 0 \\
0, & 0, & 1, & 0 \\
0, & 0, & 0, & 1
\end{array}\right|
$$

Therefore, we can compute the Jones projection $p_{J_{20}}$ right away by inverting its coproduct formula (otherwise, we should have orthogonalized it first by Gramm-Schmidt, and use a variant of the formula involving the norms):

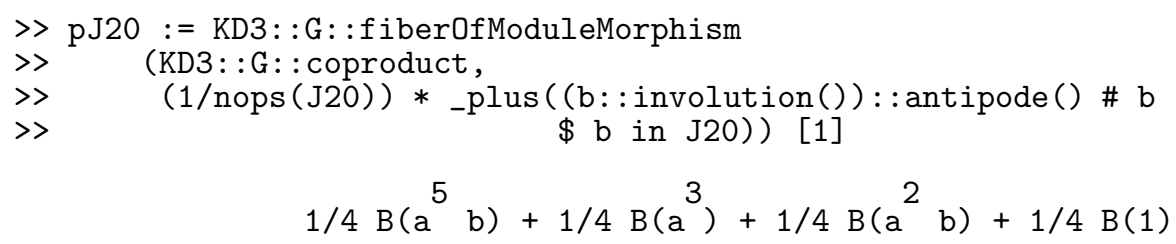


Now the construction of $p_{J_{20}}$ (and of $p_{J_{0}}$ and $p_{J_{2}}$ ) is obvious, and can be right away generalized to obtain the coideal subalgebras of dimension 4 in $K D(2 n+1)$ (see 5.11, 6.7, and 6.9.1). Beware however that the correspondence we used between coideals of dimension $d$ of $K D(3)$ and those of dimension $\operatorname{dim} K D(3) / d$ is only defined up to an automorphism of $K D(3)$.

B.5.4. The isomorphism between $K D(2 n)$ and $K Q(2 n)$. The lattice of coideal subalgebras of $K D(3)$ being complete, we now show how parts of the lattice for the larger algebras can be build up from that of the smaller ones (see 7.7). Let us start by defining $K D(6)$ :

$>\mathrm{KD} 6:=\mathrm{KD}(6)$ :

We know that $K D(3)$ embeds as $K_{4}$ in $K D(6)$. Let us show how to obtain the coideal subalgebras of $K_{4}$ from those of $K D(3)$. We have to be a bit careful as, by default, $K D(3)$ and $K D(6)$ are not defined over the same ground field (unless stated otherwise, the ground field for $K D(n)$ is $\mathbb{Q}(i, \epsilon)$ where $\epsilon$ is a $2 n$-th root of unity). Here, we force $K D(3)$ to use the same field as $K D(6)$.

> KD3 := KD(3, KD6: : coeffRing):

To ease the notations, we define shortcuts for the algebra generators of $K D(3)$ and $K D(6)$ :

$\gg[\mathrm{a} 3, \mathrm{b3}]:=\mathrm{KD} 3:: \mathrm{G}:$ :algebraGenerators: :list():

> [a6, b6] := KD6::G::algebraGenerators::list():

Now we can define the embedding of $K D(3)$ as $K_{4}$ in $K D(6)$ (see 5.4.2):

> KD3ToKD6 := KD3: :G: :algebraMorphism(table $\left.\left(\mathrm{a}=\mathrm{a} 6^{\wedge} 2, \mathrm{~b}=\mathrm{b} 6\right)\right)$ :

and use it as follows:

$>\operatorname{KD} 3 T o K D 6\left(1+2 * a 3^{\wedge} 3+3 * \mathrm{~b} 3\right)$

$$
2 B\left(a^{6}\right)+3 B(b)+B(1)
$$

We now take the Jones projection of the coideal $J_{20}$ in $K D(3)$ (see Appendix B.5.3), and lift it into $K D(6)$ :

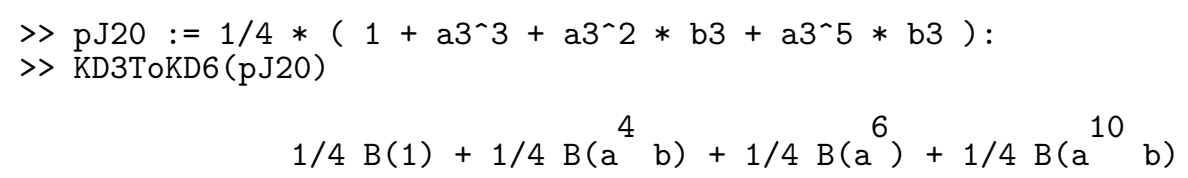

The result is a posteriori obvious, but the point is that this construction is completely automatic.

In a similar vein, one can obtain all the coideal subalgebras of $K_{3}$, as it is $K Q(3)$ via the isomorphism between $K D(2 n)$ and $K Q(2 n)$ (see Theorem 8.2). This isomorphism was first suspected by comparing the representation theory of the algebras, their dual, and the properties of the simple coideal subalgebras. The systematic computation of the isomorphisms for $n \leq 3$ suggested the general formulas which then were checked for $n \leq 10$ :

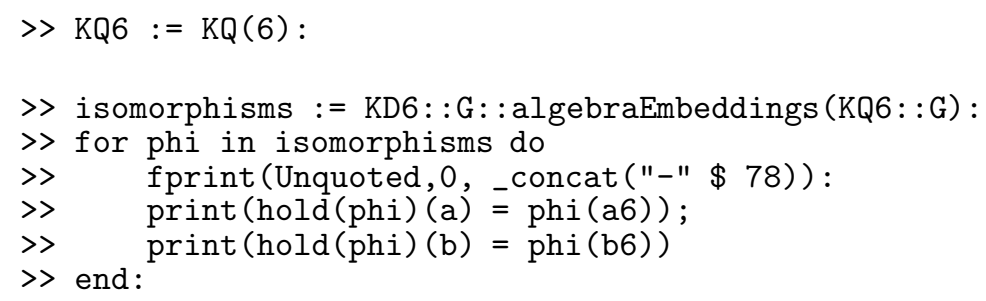




$$
\begin{gathered}
\operatorname{phi}(a)=3 / 4 B(a)+1 / 4 B\left(a^{2} b\right)+-1 / 4 B\left(a^{4} b\right)+-1 / 4 B\left(a^{5}\right)+1 / 4 B\left(a^{7}\right)+ \\
-1 / 4 B\left(a^{8} b\right)+1 / 4 B\left(a^{10} b\right)+1 / 4 B\left(a^{11}\right) \\
\operatorname{phi}(b)=-\frac{I I}{2} B\left(a^{3}\right)+1 / 2 B\left(a^{3} b\right)+\frac{I I}{2} B\left(a^{9}\right)+1 / 2 B\left(a^{9} b\right)
\end{gathered}
$$

The isomorphism we chose in Theorem 8.2 is now built into the system:

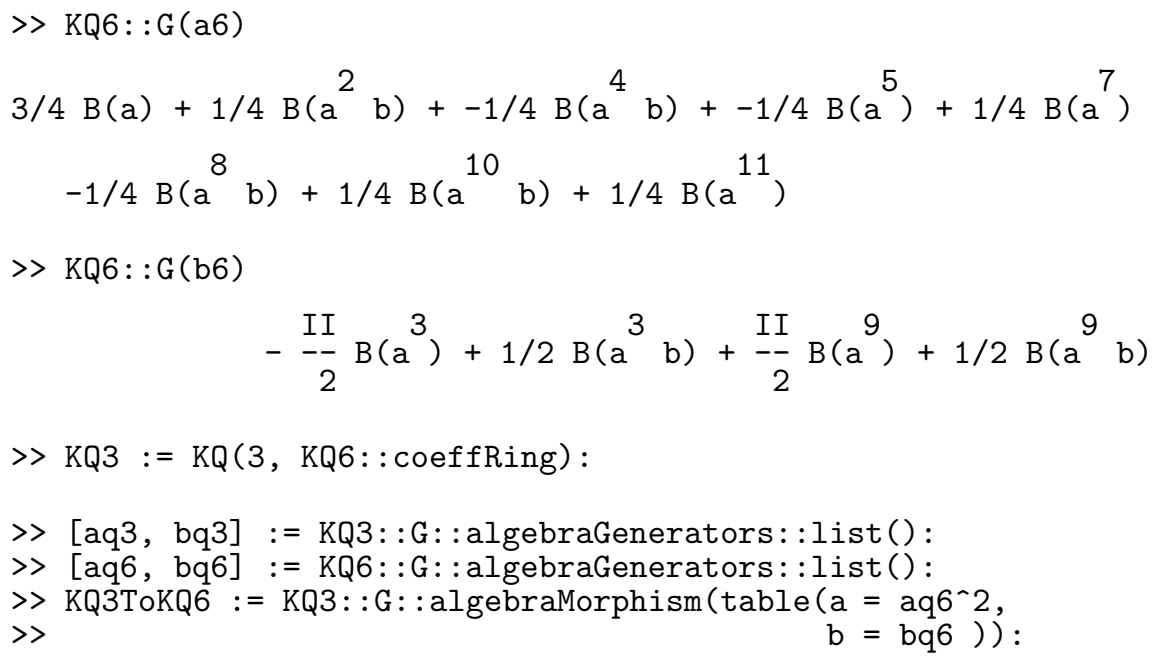

We can now conclude by demonstrating the lifting of the 4-dimensional coideal $I=$ $\delta\left(K_{21}\right)$ of $K Q(3)$ to a coideal $J_{5}$ of $K D(6)$ (see 7.7.3). Here is the Jones projection of $\delta\left(K_{21}\right)$ (see 8.4.3):

> pI :=KQ3::e(1)+KQ3::q(0,2*PI/3,2):

and its image in $K D(6)$ :

> pIInKD6 := KQ3ToKQ6 (KQ3::G(pI))

$$
1 / 4 \mathrm{~B}(1)+1 / 4 \mathrm{~B}(\mathrm{a} b)+1 / 4 \mathrm{~B}\left(\mathrm{a}^{6}\right)+1 / 4 \mathrm{~B}\left(\mathrm{a}^{7} \mathrm{~b}\right)
$$

Again, the result is trivial, and the expression in the matrix basis of 7.7 .3 can be obtained with:

>> KQ6: :M(pIInKD6)

As expected, this projection generates a coideal subalgebra of dimension 4:

> nops (coidealAndAlgebraClosure([pIInKD6])), 1/pIInKD6: :traceNormalized()

$$
4,4
$$

B.6. Further directions. Putting everything together, it is completely automatic to test whether a projection is the Jones projection of a coideal subalgebra, and to construct new Jones projections from previous ones by various techniques: embeddings, self-duality, intersection and (completed) union, etc. In the small examples we considered, this was sufficient to actually construct semi-automatically all coideal subalgebras. Furthermore, given the Jones projection of two coideal subalgebras $A \subset B$, it would be straightforward to compute the Bratelli diagram of inclusion (this is not yet implemented). The ultimate goal would be to compute automatically all the Jones projections, and therefore the full lattice of coideal subalgebras, but we do not know yet how to achieve this. 


\section{REFERENCES}

[Asc08] Michael Aschbacher. On intervals in subgroup lattices of finite groups. J. Amer. Math. Soc., 21(3):809-830, 2008.

[BBS99] S. Baaj, E. Blanchard, and G. Skandalis. Unitaires multiplicatifs en dimension finie et leurs sous-objets. Ann. Inst. Fourier (Grenoble), 49(4):1305-1344, 1999.

[Bis94a] Dietmar Bisch. An example of an irreducible subfactor of the hyperfinite $\mathrm{II}_{1}$ factor with rational, noninteger index. J. Reine Angew. Math., 455:21-34, 1994.

[Bis94b] Dietmar Bisch. A note on intermediate subfactors. Pacific J. Math., 163(2):201-216, 1994.

[BNS99] Gabriella Böhm, Florian Nill, and Kornél Szlachányi. Weak Hopf algebras. I. Integral theory and $C^{*}$-structure. J. Algebra, 221(2):385-438, 1999.

[CDMM04] C. Călinescu, S. Dăscălescu, A. Masuoka, and C. Menini. Quantum lines over noncocommutative cosemisimple Hopf algebras. J. Algebra, 273(2):753-779, 2004.

[Dav96] Marie-Claude David. Paragroupe d'Adrian Ocneanu et algèbre de Kac. Pacific J. Math., 172(2):331-363, 1996.

[Dav05] Marie-Claude David. $C^{*}$-groupoïdes quantiques et inclusions de facteurs: structure symétrique et autodualité, action sur le facteur hyperfini de type $\mathrm{II}_{1}$. J. Operator Theory, 54(1):27-68, 2005.

[Dav09] Marie-Claude David. Action d'une paire de $C^{*}$-groupoïdes quantiques finis réguliers en dualité sur une tour de facteurs hyperfinis de type $\mathrm{II}_{1}$. Appendix A of Dav05] in arXiv:math/0306374v4 [math.QA], 2009.

[dBG91] Jan de Boer and Jacob Goeree. Markov traces and $\mathrm{II}_{1}$ factors in conformal field theory. Comm. Math. Phys., 139(2):267-304, 1991.

[ES92] Michel Enock and Jean-Marie Schwartz. Kac algebras and duality of locally compact groups. Springer-Verlag, Berlin, 1992. With a preface by Alain Connes, With a postface by Adrian Ocneanu.

[EV96] Michel Enock and Leonid Vaŭnerman. Deformation of a Kac algebra by an abelian subgroup. Comm. Math. Phys., 178(3):571-596, 1996.

[GdlHJ89] Frederick M. Goodman, Pierre de la Harpe, and Vaughan F. R. Jones. Coxeter graphs and towers of algebras, volume 14 of Mathematical Sciences Research Institute Publications. SpringerVerlag, New York, 1989.

[HS96] Jeong Hee Hong and Wojciech Szymański. On finite subfactors with principal graph $D_{2 n+1} / \mathbf{Z}_{2}$. J. Funct. Anal., 141(2):294-300, 1996.

[HT04] Florent Hivert and Nicolas M. Thiéry. MuPAD-Combinat, an open-source package for research in algebraic combinatorics. Sém. Lothar. Combin., 51:Art. B51z, 70 pp. (electronic), 2004. http://mupad-combinat.sf.net/.

[IK02] Masaki Izumi and Hideki Kosaki. Kac algebras arising from composition of subfactors: general theory and classification. Mem. Amer. Math. Soc., 158(750):198, 2002.

[Jon83] V. F. R. Jones. Index for subfactors. Invent. Math., 72(1):1-25, 1983.

[JS97] V. Jones and V. S. Sunder. Introduction to subfactors, volume 234 of London Mathematical Society Lecture Note Series. Cambridge University Press, Cambridge, 1997.

[Kas95] Christian Kassel. Quantum groups, volume 155 of Graduate Texts in Mathematics. SpringerVerlag, New York, 1995.

[KP66] G. I. Kac and V. G. Paljutkin. Finite ring groups. Trudy Moskov. Mat. Obšč., 15:224-261, 1966.

[Mas00] Akira Masuoka. Cocycle deformations and Galois objects for some cosemisimple Hopf algebras of finite dimension. In New trends in Hopf algebra theory (La Falda, 1999), volume 267 of Contemp. Math., pages 195-214. Amer. Math. Soc., Providence, RI, 2000.

[Nik98] Dmitri Nikshych. $K_{0}$-rings and twisting of finite-dimensional semisimple Hopf algebras. Comm. Algebra, 26(1):321-342, 1998.

[NV00a] Dmitri Nikshych and Leonid Vainerman. Algebraic versions of a finite-dimensional quantum groupoid. In Hopf algebras and quantum groups (Brussels, 1998), volume 209 of Lecture Notes in Pure and Appl. Math., pages 189-220. Dekker, New York, 2000.

[NV00b] Dmitri Nikshych and Leonid Vainerman. A characterization of depth 2 subfactors of $\mathrm{II}_{1}$ factors. J. Funct. Anal., 171(2):278-307, 2000.

[NV00c] Dmitri Nikshych and Leonid Vainerman. A Galois correspondence for II $_{1}$ factors and quantum groupoids. J. Funct. Anal., 178(1):113-142, 2000. 
[NV02] Dmitri Nikshych and Leonid Vainerman. Finite quantum groupoids and their applications. In New directions in Hopf algebras, volume 43 of Math. Sci. Res. Inst. Publ., pages 211-262. Cambridge Univ. Press, Cambridge, 2002.

[Pop90] Sorin Popa. Sur la classification des sous-facteurs d'indice fini du facteur hyperfini. C. R. Acad. Sci. Paris Sér. I Math., 311(2):95-100, 1990.

[PP86] Mihai Pimsner and Sorin Popa. Entropy and index for subfactors. Ann. Sci. École Norm. Sup. (4), 19(1):57-106, 1986.

$\left[\mathrm{S}^{+} 09\right] \quad$ W. A. Stein et al. Sage Mathematics Software (Version 3.3). The Sage Development Team, 2009. http://www. sagemath.org.

[SCc08] The Sage-Combinat community. Sage-Combinat: enhancing Sage as a toolbox for computer exploration in algebraic combinatorics, 2008. http://combinat.sagemath.org.

[The96] The MuPAD Group, Benno Fuchssteiner et al. MuPAD User's Manual - MuPAD Version 1.2.2. John Wiley and sons, Chichester, New York, first edition, march 1996. includes a CD for Apple Macintosh and UNIX.

[Vai98] Leonid Vainerman. 2-cocycles and twisting of Kac algebras. Comm. Math. Phys., 191(3):697721, 1998.

[Wat90] Yasuo Watatani. Index for $C^{*}$-subalgebras. Mem. Amer. Math. Soc., 83(424):vi+117, 1990.

[Wat96] Yasuo Watatani. Lattices of intermediate subfactors. J. Funct. Anal., 140(2):312-334, 1996.

E-mail address: mcld@math.u-psud.fr

E-mail address: Nicolas.Thiery@u-psud.fr

Univ Paris-Sud, Laboratoire de Mathématiques d'Orsay, Orsay, F-91405; CNRS, Orsay, F-91405 